\title{
Biomedical signal analysis in automatic classification problems
}

ELIES FUSTER I GARCIA 


\section{BIOMEDICAL SIGNAL ANALYSIS IN AUTOMATIC \\ CLASSIFICATION PROBLEMS}

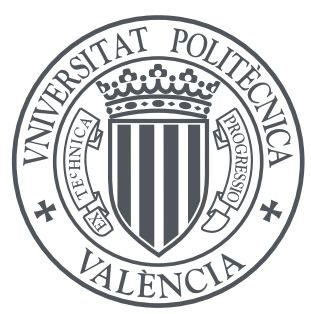

UNIVERSITAT POLITÈCNICA DE VALÈNCIA DEPT. FÍSICA APLICADA

DOCTORAL THESIS

PRESENTED BY:

ELIES FUSTER I GARCIA

SUPERVISED BY:

DR. JUAN MIGUEL GARCÍA-GÓMEZ AND

DR. MONTSERRAT ROBLES VIEJO 


\section{This editorial is member of the UNE, which guarantees

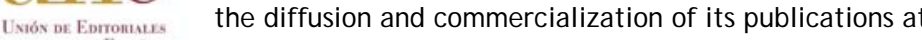 vivenstrans Exproous national and international level.}

\section{(c) Elies Fuster i Garcia}

First Edition, 2012

(C) of the present edition:

Editorial Universitat Politècnica de València www. editorial. upv.es

ISBN: -978-84-8363-619-0 (printed version)

Any unauthorized copying, distribution, marketing, editing, and in general any other exploitation, for whatever reason, of this piece of work or any part thereof, is strictly prohibited without the authors' expressed and written permission. 
Aquesta tesi no hauria sigut possible sense el suport, la confiança i la generositat de tot un conjunt de companys i amics als quals m'agradaria dedicar aquest treball.

En primer lloc voldria agrair als meus directors, el Dr. Juan Miguel García Gómez i la Dra. Montse Robles Viejo, el haver-me oferit l'oportunitat de treballar en el camp de la informàtica biomèdica. A ells els dec no sols la direcció d'aquest treball i gran part dels meus coneixements en aquest camp, sinó haver sigut per a mi uns grans referents professionals i personals.

Al llarg d'aquests últims anys he tingut el plaer de compartir cada dia amb els companys del grup d'informàtica biomèdica IBIME. En concret, voldria fer un agraïment especial a: Salvador Tortajada i Javier Vicente, per compartir aquest procés i per tot el suport moral que m'han donat davant cada entrebanc que ha sorgit; a tots els companys i companyes que han format part del grup de reconeixement de patrons al llarg d'aquest període, patint els meus acudits i compartint discussions de cafè: Carlos Sáez, Miguel Esparza, Adrián Bresó, Javier Juan, Alfonso Pérez, Alfredo Navarro, Patricia Paniagua, Míriam González i Ana Ferrer; i, finalment, al Dr. José Vicente Manjón, per compartir amb mi les seues idees i per l'ajuda que m'ha oferit amb els tecnicismes de l'anàlisi d'imatges.

Els meus inicis en el camp de la recerca no haurien sigut possibles sense el suport del Dr. Lluís Miquel Garcia Raffi, del Dr. Juan V. Sánchez Pérez i del Dr. Enrique Sánchez Pérez, al Centre de Tecnologies Físiques (CTFAMA). Gràcies a ells he pogut dedicar-me a la recerca i començar el camí que ha fet possible aquest treball. També, i de forma particular, m'agradaria agrair al meu amic, el Dr. Vicent Romero, el fet d'haver compartit anys de despatx, viatges i aventures vàries en els primers anys de recerca.

Voldria agrair al Dr. Franklyn A. Howe l'oportunitat de fer una estada sota la seua direcció al centre St George's, University of London. Al St. George's he tingut el plaer de compartir despatx, feina i bons moments amb Felix Raschke. També voldria agrair a l'equip de professors de la Universitat Internacional Valenciana, i en particular a l'àrea d'astronomia i astrofísica, el suport que m'han donat al llarg del meu període com a docent en aquesta Universitat.

Aquesta tesi ha sigut desenvolupada parcialment en el context dels projectes de la Comissió Europea eTUMOUR (contracte núm. FP6-2002LIFESCIHEALTH 503094) i HEALTHAGENTS (contracte núm. FP6-2005IST 027213), així com en diversos projectes d'àmbit estatal com DSSPROSTATA (FIT-340001-2007-14), DSSPROSTATA2 (TSI-020302-2009-65) i Sistema Expert en Electrocardiografia (TSI-020302-2010-136). Voldria agrair als consorcis d'aquests projectes, al conjunt de hospitals que hi han contribuit amb dades biomèdiques, i a tota la gent amb la qual he treballat directament, tots aquell coneixements que hem compartit al llarg d'aquests anys. En espe- 
cial voldria fer esment d'aquelles persones que han contribuït de forma més significativa al desenvolupament d'aquest treball amb el seu suport, els seus coneixements i la seua dedicació: la Prof. Sabine Van Huffel (Katholieke Universiteit Leuven), el Dr. Luis Martí Bonmatí i el Dr. Àngel Bayarri (Quirón València), la Dra. Magdalena Rafecas, el Dr. Jorge Cabello i el Dr. Josep Oliver (Universitat de València).

Com no podria ser d'altra manera, voldria dedicar de forma especial aquesta tesi a la meua família. Primerament als meus pares, Josep i Amèlia. A ells els dec ser com sóc i tindre els valors en què crec. Mai podré agrairlos prou l'esforç que han fet per permetre'm recórrer aquest llarg camí. A la meua germana Núria, per animar-me sempre a continuar lluitant per allò en què crec. Als meus oncles i cosins, que fan que mai em senta sol. A Inés i Thomas per l'ajuda amb les correccions. Als que ja no hi són, perquè ells han construït aquesta família. A Asun, Carlos, Sofia i Iban, per fer-me sentir com a casa. I finalment a Teba, la persona que sempre ha estat al meu costat donantme força per seguir endavant i fent-me sentir la persona més afortunada. És un orgull tindre una família així.

Finalment, m'agradaria dedicar la tesi doctoral als pacients que han contribuït a la ciència, incloent les seues esperances en les dades biomèdiques emprades en aquests estudis. 
Al llarg de l'última dècada hem assistit a un desenvolupament sense precedents de les tecnologies de la salut. Els avenços en la informatització, la creació de xarxes, les tècniques d'imatge, la robòtica, les micro/nano tecnologies, i la bioinformàtica, han contribuït a augmentar significativament la quantitat i diversitat d'informació a l'abast del personal clínic per al diagnòstic, pronòstic, tractament, i seguiment dels pacients. Aquest augment en la quantitat i diversitat de dades clíniques requereix del continu desenvolupament de tècniques i metodologies capaces d'integrar aquestes dades, processar-les, i donar suport en la seva interpretació d'una forma robusta i eficient.

En aquest context, aquesta tesi es focalitza en l'anàlisi i processament de senyals biomèdiques i el seu ús en problemes de classificació automàtica. És a dir, es focalitza en: el disseny i integració d'algorismes per al processament automàtic de senyals biomèdics, el desenvolupament de nous mètodes d'extracció de característiques per a senyals, l'avaluació de compatibilitat entre senyals biomèdics, i el disseny de models de classificació per a problemes clínics específics. En la majoria de casos continguts en aquesta tesi, aquests problemes es situen en l'àmbit dels sistemes de suport a la decisió clínica o sistemes computacionals que proporcionen coneixement expert per a la decisió en el diagnòstic, pronòstic i tractament dels pacients.

Una de les principals contribucions d'aquesta tesi consisteix en l'avaluació de la compatibilitat entre espectres de ressonància magnètica (ERM) obtinguts mitjançant dues tecnologies d'escàners de ressonància magnètica coexistents en l'actualitat (escàners de 1.5T i de 3T). Aquesta compatibilitat s'avalua en el context de la classificació automàtica de tumors cerebrals. Els resultats obtinguts en aquest treball suggereixen que els classificadors existents basats en ERM de 1.5T poden ser aplicables a casos adquirits amb la nova tecnologia d'ERM 3T. Aquest fet permet aprofitar l'esforç realitzat al llarg dels últims anys en la recopilació de grans conjunts de dades d'ERM i en la creació de models predictius basats en ERM de 1.5T.

Una altra contribució principal d'aquesta tesi és el disseny i implementació d'un nou mètode d'extracció de característiques, RSFDA, per a la classificació d'espectres d'ERM de tumors cerebrals. RSFDA es basa en una descripció funcional dels espectres ERM mitjançant una base de B-splines no uniformement distribuïdes. En aquest treball es demostra el bon comportament de RSFDA davant els artefactes més comuns en ERM, mitjançant una completa avaluació amb dades simulades i dos grans bases de dades multicèntriques i multiprojecte. L'aplicació d'RSFDA constitueix una interessant alternativa a altres mètodes d'extracció de característiques i en especial per aquells conjunts d'espectres que continguin altes deformacions de línia base.

D'altra banda, aquesta tesi aporta un nou mètode per a la classificació de coincidències en escàners de tomografia per emissió de positrons (TEP). 
Aquest algorisme millora el rendiment del mètode tradicional per a la detecció de coincidències basat en un finestrat temporal de coincidència i l'aplicació de condicionants geomètrics. L'algorisme dissenyat fa ús d'una xarxa neuronal artificial (XNA) entrenada amb dades simulades amb l'objectiu d'identificar coincidències vertaderes en aquest tipus d'escàners. Comparant ambdós algorismes a un mateix valor d'eficiència, l'algorisme basat en XNA presenta sempre un resultat amb menor percentatge de successos aleatoris. Així mateix, la comparació de les imatges resultants reconstruïdes mitjançant ambdós mètodes constaten una millora en totes les figures de mèrit incloses a l'estudi.

Finalment, aquesta tesi presenta un estudi multicèntric per avaluar diferents mètodes d'extracció de característiques, algoritmes de classificació, i mètodes d'alineament, per a detecció i classificació automàtica d'arítmies mitjançant senyals d'electrocardiogrames (ECG). A diferència de la majoria d'estudis publicats en aquest camp, en aquest cas s'ha aconseguit el recopilar una gran base de dades anotada i amb una configuració estàndard de 12 derivacions. Aquest estudi s'ha centrat en la classificació de batecs d'arítmies ventriculars contra batecs d'arítmies supra-ventriculars i normals. Els resultats mostren una millora en els resultats obtinguts pels classificadors quan s'utilitza la base de dades amb els batecs alineats. També s'observa el bon funcionament dels senzills algorismes de KNN per a aquest tipus de problemes de classificació.

Els resultats d'aquesta tesi han contribuit directament als projectes: eTUMOUR (VI Framework Program, LSHC-CT-2004-503094, 2004-2009), HealthAgents (VI Framework Program, IST-2004-27214, 2006-2009), DSSPROSTATA ( PROFIT FIT-340001-2007-14, 2007-2009), DSSPROSTATA2 (AVANZA, TSI-020302 a 2009-65, 2009-2010), i Sistema Expert en electrocardiografia (AVANZA R + D, TSI-020302-2010-136, 2010-2011). Com a resultats translacionals a partir de les contribucions d'aquesta tesi cal destacar el disseny i implementació de: (1) un pre-processat semiautomàtic d'ERM en CURIAM-BT (un sistema de suport a la decisió clínica per al diagnòstic de tumors cerebrals), (2) El disseny i implementació d'ArrythLab un programari per a pre-processat automàtic, control de qualitat, segmentació i etiquetatge de polsos en estudis d'arítmia basats en ECG, i (3) El disseny i implementació de MRSILab, una llibreria de codi lliure per MATLAB per al pre-procés, anàlisi i visualització automàtic d'imatges espectroscòpiques de ressonància magnètica (IERM). 


\section{SÍNTESIS}

A lo largo de la última década hemos asistido a un desarrollo sin precedentes de las tecnologías de la salud. Los avances en la informatización, la creación de redes, las técnicas de imagen, la robótica, las micro/nano tecnologías, y la bioinformática, han contribuido a aumentar significativamente la cantidad y diversidad de información al alcance del personal clínico para el diagnóstico, pronóstico, tratamiento y seguimiento de los pacientes. Este aumento en la cantidad y diversidad de datos clínicos requiere del continuo desarrollo de técnicas y metodologías capaces de integrar estos datos, procesarlos, y dar soporte en su interpretación de una forma robusta y eficiente.

En este contexto, esta tesis se focaliza en el análisis y procesado de señales biomédicas y su uso en problemas de clasificación automática. Es decir, se focaliza en: el diseño e integración de algoritmos para el procesado automático de señales biomédicas, el desarrollo de nuevos métodos de extracción de características para señales, la evaluación de compatibilidad entre señales biomédicas, y el diseño de modelos de clasificación para problemas clínicos específicos. En la mayoría de casos contenidos en esta tesis, estos problemas se sitúan en el ámbito de los sistemas de apoyo a la decisión clínica, esto es, sistemas computacionales que proporcionan conocimiento experto para la decisión en el diagnóstico, pronóstico y tratamiento de los pacientes.

Una de las principales contribuciones de esta tesis consiste en la evaluación de la compatibilidad entre espectros de resonancia magnética (ERM) obtenidos mediante dos tecnologías de escáneres de resonancia magnética coexistentes en la actualidad (escáneres de 1.5T y de 3T). Esta compatibilidad se evalúa en el contexto de clasificación automática de tumores cerebrales. Los resultados obtenidos en este trabajo sugieren que los clasificadores existentes basados en datos de ERM de 1.5T pueden ser aplicables a casos obtenidos con la nueva tecnología de ERM 3T. Esta conclusión permite aprovechar el esfuerzo realizado a lo largo de los últimos años en la recopilación de grandes bases de datos de ERM y en la creación de modelos predictivos basados en ERM de 1.5T.

Otra contribución principal de esta tesis es el diseño e implementación de un nuevo método de extracción de características, RSFDA, para la clasificación de espectros de ERM de tumores cerebrales. RSFDA se basa en una descripción funcional de los espectros ERM mediante una base de B-splines no uniformemente distribuidas. En este trabajo se demuestra el buen comportamiento de RSFDA frente a los artefactos más comunes en ERM, mediante una completa evaluación utilizando datos simulados y dos grandes bases de datos multicéntricas y multiproyecto. La aplicación de RSFDA constituye una interesante alternativa a otros métodos de extracción de características y en especial para aquellos conjuntos de espectros que contengan altas deformaciones de línea base. 
Por otro lado, esta tesis aporta un nuevo método para la clasificación de coincidencias en escáneres de tomografía por emisión de positrones (TEP). Este algoritmo mejora el rendimiento del método tradicional para la detección de coincidencias basado en un ventanado temporal de coincidencia y la aplicación de condicionantes geométricos. El algoritmo diseñado hace uso de una red neuronal artificial (RNA) entrenada con datos simulados con el objetivo de identificar coincidencias verdaderas en este tipo de escáneres. Comparando ambos algoritmos a un mismo valor de eficiencia, el algoritmo basado en (RNA) presenta siempre un resultado con menor porcentaje de sucesos aleatorios. Así mismo, la comparación de las imágenes resultantes reconstruidas mediante los distintos métodos constatan una mejora en todas la figuras de mérito incluidas en el estudio.

Finalmente, esta tesis presenta un estudio muticéntrico para la evaluación de distintos métodos de extracción de características, algoritmos de clasificación, y métodos de alineamiento, para detección y clasificación automática de arritmias mediante señales de electrocardiogramas (ECG). A diferencia de la mayoría de estudios publicados al respecto, en este caso se ha logrado el recopilar una base de datos con un número de casos anotados relativamente grande, y con una configuración estándar de 12 derivaciones. Este estudio se ha centrado en la clasificación de latidos de arritmias ventriculares contra latidos de arritmias supra-ventriculares y normales. Los resultados muestran una mejoría en los resultados obtenidos por los clasificadores, cuando se utiliza la base de datos con los latidos alineados. También se observa el buen funcionamiento de los sencillos algoritmos de KNN para este tipo de problemas de clasificación.

Los resultados de esta tesis han contribuido directamente a los proyectos: eTUMOUR (VI Framework Program, LSHC-CT-2004-503.094, 2004-2009), HealthAgents (VI Framework Program, IST-2004-27.214, 2006-2009), DSSPROSTATA (PROFIT FIT-340001-2007-14, 2007-2009), DSSPROSTATA2 (AVANZA, TSI-020302 a 2009-65, 2009-2010), y Sistema Experto en electrocardiografía (AVANZA I + D, TSI-020302-2010-136, 2010-2011). Como resultados translacionales a partir de las contribuciones de esta tesis cabe destacar el diseño e implementación de: (1) un pre-procesado semiautomático de ERM en CURIAM-BT (un sistema de soporte a la decisión clínica para el diagnostico de tumores cerebrales); (2) El diseño e implementación de ArrythLab un software para pre-procesado automático, control de calidad, segmentación y etiquetado de pulsos en estudios de arritmia basados en ECG, y (3) El diseño e implementación de MRSILab, una librería de código libre para MATLAB para el pre-proceso automático, análisis y visualización de imágenes espectroscópicas de resonancia magnética (IERM). 
During the last decade an unprecedented development of healthcare technologies has taken place. Advances in computerization, networking, imaging, robotics, micro/nano technologies, and bioinformatics, have contributed to significantly increasing the amount and diversity of information available to physicians for medical diagnosis, prognosis, treatment and monitoring. The increase in the amount and diversity of clinical data requires the continuous development of new techniques and procedures capable of integrating and analysing the complexity of the whole information generated as well as providing support in their interpretation.

In this context, this thesis has been focused on the analysis and processing of biomedical signals and their use in automatic classification problems. That is, the design and implementation of automatic biomedical signal preprocessing algorithms, the assessment of compatibility between biomedical signals, the design of novel feature extraction methods for biomedical signals, and the design of classification models for specific clinical problems. In most of the cases presented, these methods will be applied in the framework of clinical decision systems; computational systems for the decision support in the diagnosis, prognosis and treatment of patients.

One of the main contributions of this thesis consists of the evaluation of the compatibility between magnetic resonance spectra (MRS) obtained by two coexisting magnetic resonance scanner technologies (1.5T and 3T) in the context of brain tumour automatic classification. The results obtained in this work suggest that existing classifiers based on $1.5 \mathrm{~T}$ datasets may be applicable to 3T 1H SV-MRS dataset. The compatibility between the now coexisting clinical MR scanners technologies of $1.5 \mathrm{~T}$ and 3T plays a decisive role in the development of new classifiers for tumour diagnosis support and for the use of the existing ones based on 1.5T. Furthermore, this will make it possible to take more benefit from the effort made throughout years in the compilation of large MRS databases.

Another contribution of this thesis is the design and implementation of a new feature extraction method, RSFDA, for the classification of brain tumour MRS spectra. RSFDA is based on a functional description or MRS data by a non-uniformly distributed B-spline basis. The evaluation of RSFDA using simulated datasets and two large real muticenter datasets, shows that RSFDA had exhibited robustness against the most common artefacts in MRS. The application of RSFDA constitutes an interesting alternative to other FE methods, especially when using datasets with high baseline deformations. Furthermore, this thesis provides a new method for coincidence classification in positron emission tomography (PET) scanners. The presented method uses an artificial neural network (ANN) trained with simulated datasets, for the identification of true coincidences. This algorithm improved the performance of traditional coincidence sorting algorithm based 
on a time coincidence window and the application of geometrical conditions. Comparing both methods at the same efficiency values, the ANN based method always presents a sorted output with a smaller random fraction. Moreover the comparison of reconstructed images using the different methods for coincidence classification, shows an improvement of all figures of merit defined in the study.

Finally, this thesis presents a multicenter study for the assessment of different feature extraction methods, classification algorithms, and aligning methods for arrhythmia automatic detection and classification by electrocardiography (ECG) signals. In this case it has been achieved to compile a relatively large dataset consisting on annotated 12-lead ECG records. This study has been focused on the classification of ventricular arrhythmia versus supra ventricular and control beats. The results show an improvement of the classification performance when using the algorithm for the beat alignment. Moreover, it is remarkable that the simplest classifiers based on k-nearest neighbor algorithm obtain the best performances.

The results of this $\mathrm{PhD}$ Thesis apply directly to the results of the following projects: eTumour (VI Framework Program, LSHC-CT-2004503094, 2004-2009), HealthAgents (VI Framework Program, IST- 200427214, 2006-2009), DSSPROSTATA (PROFIT FIT- 340001-2007-14, 20072009), DSS PROSTATA2 (AVANZA, TSI-020302-2009-65, 2009-2010), and Sistema Experto en Electrocardiografía (AVANZA I+D, TSI-020302-2010136, 2010-2011). Based on the scientific contributions studied in this thesis, different practical solutions have been developed including the design and implementation of: (1) semi-automatic pre-processing pipeline for 1H-MRS signals in CURIAM-BT (a clinical decision support system for brain tumours diagnosis by MRS); (2) ArrythLab, a software for automatic pre-processing, quality control, segmentation and beat labeling in arrhythmia studies by ECG; and (3) MRSILab, an open source MATLAB toolbox for automatic preprocessing, analysis and visualization of magnetic resonance spectroscopy imaging (MRSI) data. 
Contents xiii

I INTRODUCTION AND THEORETICAL FRAMEWORK 1

1 INTRODUCTION 3

1.1 Motivation 3

1.2 Goals 3

1.3 Contributions 5

1.4 Projects and Partners 7

1.5 Contents 9

2 BIOMEDICAL SIGNALS AND THE CLASSIFICATION PROBLEM 13

2.1 Introduction 13

2.2 Nuclear magnetic resonance spectroscopy 13

2.2.1 The nucleus under a magnetic field 13

2.2.2 Excitation and signal generation 16

2.2.3 The free induction decay and the spin-echo 18

2.2.4 Spatial localization of magnetic resonance signals 19

2.2.5 K-space and magnetic resonance image formation 24

2.2.6 Spectroscopy 25

2.3 Electrocardiography 31

2.3.1 The leads 32

2.3.2 The electrocadiogram signal 34

2.4 Positron emission tomography 36

2.4.1 Coincidence detection 37

2.4.2 Image reconstruction 38

2.5 Classification of biomedical signals 40

2.5.1 Feature extraction and selection methods 41

2.5.2 Pattern recognition based classifiers 44

2.5.3 Evaluation strategies 46

2.5.4 Figures of merit 47

II CONTRIBUTIONS 49

3 BIOMEDICAL SIGNAL PROCESSING AND VISUALIZATION 51

3.1 Design and implementation of MRSIlab 52

3.1.1 Motivation 52

3.1.2 MRSILab modules 52

3.1.3 Reading module 54

3.1.4 Magnetic Resonance Spectroscopic Imaging (MRSI) preprocessing module 61

3.1.5 FID preprocessing module 64

3.1.6 MRI-MRSI co-registration module 64 

3.1.7 Quantification module
66
3.1.8 The graphical user interface 66
3.1.9 Application 69

3.2 SV-MRS automatic pre-processing pipeline for CURIAMBT CDSS $\quad 70$

3.2.1 Motivation 70

3.2.2 SV-MRS pre-processing pipeline 71

3.3 Design and implementation of Arrhythlab 76

3.3.1 Motivation 76

3.3.2 Pre-processing 77

3.3.3 QRS detection and segmentation $\quad 77$

3.3.4 Beat editing and labeling 77

3.3.5 The graphical user interface $\quad 79$

3.3.6 Application 80

4 COMPATIBILITY BETWEEN $3 \mathrm{~T}$ AND $1.5 \mathrm{~T}$ SV-MRS BASED

CLASSIFIERS. $\quad 83$

4.1 Introduction 83

4.2 Materials and methods 85

4.2.1 In vivo ${ }^{1} \mathrm{H} \mathrm{SV}$-MRS datasets 85

4.2.2 MRS processing 86

4.2.3 Feature extraction method 88

4.2.4 Classifiers 88

4.2.5 Performance Measures 88

4.2.6 Statistical Analysis 89

4.3 Results 89

4.4 Discussion 92

5 EXTRACTING MRS DISCRIMINANT FUNCTIONAL FEATU-

RES 95

5.1 Introduction 95

5.2 Materials 97

5.2.1 Simulated MRS dataset 97

5.2.2 Real multicenter brain tumor MRS dataset 102

5.3 Functional Data Analysis based on region segmentation 103

5.3.1 Spectra processing 104

5.3.2 Generation of seeds 104

5.3.3 Region segmentation 107

5.3.4 Region modeling with functional description 107

5.4 Experimental strategies 109

5.4.1 Classification methods 112

5.4.2 FE methods used for comparison 113

5.4.3 Evaluation metrics 113

5.5 Results 113

5.5.1 Simulated MRS dataset 114

5.5.2 Multicenter brain tumor MRS dataset 118

5.6 Discussion 118 
6 REDUCTION OF RANDOM COINCIDENCES IN PET 123

6.1 Introduction 124

6.2 Methods 124

6.2.1 Monte-Carlo simulations 124

6.2.2 Coincidence sorters 126

6.2.3 Analysis 127

6.2.4 Reconstruction algorithm and image analysis 128

6.3 Results \& Discussion 129

6.4 Conclusion \& Future work 130

7 AUTOMATIC CLASSIFICATION OF ARRHYTHMIAS BASED ON ECG 135

7.1 Introduction 135

7.2 Materials: The SEE database 136

7.3 Methods 137

7.3.1 Pre-processing, QRS detection, and beat labeling 137

7.3.2 Quality Control 137

7.3.3 Beat alignment 140

7.3.4 Dimensionality reduction based on prior knowledge 141

7.3.5 Feature extraction 141

7.3.6 Classifiers 141

7.3.7 Evaluation strategy 142

7.4 Results 142

7.4.1 Patient dependence analysis 142

7.4.2 Number of variables 144

7.4.3 Effect of alignment 144

7.4.4 Feature extraction and classification algorithms 145

7.5 Discussion 145

7.6 Conclusion 148

III CONCLUSIONS 149

8 CONCLUDING REMARKS AND FUTURE WORK 151

8.1 Conclusions 151

8.2 Future work 152

IV APPENDIX 155

A B-SPLINES 157

A.1 The B-Splines basis 157

A.2 Coefficient estimation 159

A.2.1 Unweighted least squares fits 159

A.2.2 Weighted least squares fits 159

A.2.3 Weighted least squares fits with a roughness penalty 161

List of Figures 165

List of Tables 169

Bibliography 171 

ACC Accuracy

Ala Alanine

ANN Artificial Neural Networks

CDSS Clinical Decision Support Systems

Cho Choline

cb city block

Cr Creatine

CS Chemical Shift

CG2G Common Grade II Glial- astrocytomas grade II, oligoastrocytoma and oligodendroglioma -

CSI Chemical Shift Imaging

CV Cross Validation

dDWH distributed Data Warehouse

E Efficiency

ECC Eddy Current Correction

ECG Electrocardiogram

eTUMOUR EC project, contract no. FP6-2002-LIFESCIHEALTH 503094

FC Fisher Criterion

FE Feature Extraction

FID Free Induction Decay

FFT Fast Fourier Transform

FOV Field of View

FS Feature Selection

G Geometric Mean of Recalls

GBM Glioblastoma

Gly Glycine 
Glx Glutamate + Glutamine

GUI Graphical User Interface

HGM High Grade Malignant

HLSVD Hankel-Lanczos singular value decomposition

ICA Independent Component Analysis

i.i.d. independent and identically distributed

INTERPRET EC project, contract no. FP5-1999-IST-10310

KNN K-Nearest Neighbors

L1 Lipid resonance at 1.29 parts per million (ppm)

L2 Lipid resonance at $0.92 \mathrm{ppm}$

Lac Lactate

LDA Linear Discriminant Analysis

LOR Line-of-Response

LTE Long Time Echo

MEN low-grade Meningiomas

MET Metastases

mI Myo-Inositol

ML Machine Learning

MLP Multilayer Perceptron

MR (Nuclear) Magnetic Resonance

MRI Magnetic Resonance Imaging

MRS Magnetic Resonance Spectroscopy

MRSI Magnetic Resonance Spectroscopic Imaging

MSE Mean Squared Error

MVS Muti-voxel Spectroscopy

NAA N-Acetyl Aspartate

NAc N-Acetyl groups

NMR Nuclear Magnetic Resonance

PCA Principal Components Analysis 
PENSSE Penalized Least Squares Criterion

PET Positron Emission Tomography

PI Peak Integration

PR Pattern Recognition

PRESS Point Resolved Spectroscopy

ppm parts per million

PSF Point Spread Function

QDA Quadratic Discriminant Analysis

RaF Random Fraction

ReliefF Recursive elimination of Features

RF Radio Frequency

ROI Region of Interest

RSFDA Functional Data Analysis based on Region Segmentation

SEE Sistema Experto en Electrocardiografía project

SNR Signal-to-Noise Ratio

STE Short Time Echo

STEAM STimulated Echo Acquisition Mode

SV Single-Voxel

SVS Single-Voxel Spectroscopy

SW Stepwise

Tau Taurine

TE Echo Time

TR Repetition Time

ULN Unit length normalization

WAV Wavelet transform 

Part I

INTRODUCTION AND THEORETICAL FRA MEW OR K 



\subsection{MOTIVATION}

During the last decade an unprecedented development of healthcare technologies and healthcare models has taken place, contributing to significantly increasing the amount and diversity of information available to physicians for medical diagnosis, prognosis, treatment and monitoring [Gri11]. Both the increase in the amount and diversity of clinical data, and the evolution of the healthcare models, require new efficient techniques and procedures capable of integrating and analyzing the complexity of the whole information generated.

One of the main disciplines to overcome these problems is Pattern Recognition (PR) [HRM04, MdS10]. The PR is based on the study of similarities between objects features, to establish relations between sets of such objects. The development, implementation and validation of PR based systems to process and interpret clinical data, need close collaboration between physicians, biologists and biochemists, and engineers and physicists. Moreover, when these PR based systems are applied to complex data (e.g. biomedical signals and images), the inclusion of data pre-processing modules, quality control protocols, and feature extraction algorithms is mandatory.

In this context, this thesis will be focused on the design, implementation, and application of techniques for the biomedical signal analysis in the context of automatic classification problems. In most of the cases presented, these methods will be applied in the framework of clinical decision systems. That is, to develop computational systems that provide expert knowledge for decision support in the diagnosis, prognosis and treatment of patients. Specifically, this thesis compiles the research carried out in the last years in classification problems involving Magnetic Resonance Spectroscopy (MRS), MRSI, Positron Emission Tomography (PET) and Electrocardiogram (ECG) signals.

\subsection{GOALS}

The general goal of this thesis is to contribute in the development of biomedical signal analysis methods in the context of clinical classification problems, allowing among others the implementation and assessment of clinical decision support systems based on biomedical signals in the real 
clinical environment. This main goal, can however, be subdivided into several specific goals, as follows:

- G1 - Design and implementation of automatic biomedical signal processing pipelines for MRSI, ECG and MRS: In the automatic analysis of biomedical signals, the pre-processing needs to be integrated in the Clinical Decision Support Systems (CDSS). This ensures the application of the same protocols in the training signals and the ones acquired from new cases, improving the reproducibility of the results obtained and their performance. Specifically, the interest is focused on pipelines for multi-center data to pre-process MRS, MRSI and ECG signals

- G2 - Assessment of data compatibilities in the context of automatic classification problems: The effectiveness of the training of supervised classifiers is subjected to the quantity and quality of the signals used. Although different biomedical signals have been obtained using the same acquisition modality, in most cases, the technologies used for the acquisition differ because the evolution of the acquisition technologies, and it is not easy to see whether the signals obtained with both procedures could be compatible to use together or whether they are not. The study of compatibility between signals obtained using the same modality but different technologies, allows us to extend the use of the systems already trained as well as to expand the number of signals available for the training of new ones. This assessment of data compatibility is particularly interesting in the case of MRS obtained with $1.5 \mathrm{~T}$ Nuclear Magnetic Resonance (NMR) scanners and 3T NMR scanners. The compatibility between both data types, will allow the use of larger combined datasets to build more robust classifiers, and to extend the capabilities of the classifiers already trained with $1.5 \mathrm{~T}$ MRS spectra to be used with new 3T MRS cases.

- G3 - Design and implementation of new feature extraction techniques for biomedical signal classification: Although there is a wide diversity of feature extraction methods, most of them do not take into account the particular characteristics of continuous signals. The design of specific methods for different types of signals, enables the extraction of a greater part of the information contained therein, it increases the robustness against different types of artifacts characteristic of the signal modality, and in short it improves the models performance. Specifically, the interest is focused on the design of supervised feature extraction algorithms based on functional description of the biomedical signal.

- G4 - Design of coincidence-sorting algorithms for small animal PET scanners: Design of automatic analysis methods for PET detectors signals by means of supervised classification strategies to improve currently coincidence-sorting methods based on temporal windows and geometric conditions. 
- G5 - Design and evaluation of supervised classification models based on multi-center datasets for arrhythmia detection and classification by ECG: Special attention is given to the evaluation of different feature selection methods and the effectiveness of the algorithms for quality control and alignment of pulses.

\subsection{CONTRIBUTIONS}

The scientific contributions of this thesis have been published in scientific journals and proceedings in the fields of Machine Learning, Magnetic Resonance, Molecular Biology, and Medical Imaging. Moreover the technological results of this thesis have been compiled in registered software packages or toolboxes.

The most significant contributions included in this thesis can be summarized as follows:

- C1 - Design and implementation of an open source MRSI toolbox MRSILab for MATLAB . This toolbox allows the automatic processing of MRSI signals as well as their analysis and visualization. This toolbox is being registered by the Universitat Politècnica de València and was included in a virtual working environment. In this manner it is intended to promote the collaborative development of new functionalities and updates, to stimulate the questions solving, news distribution and easy communication between developers community. MRSILab has successfully been used in different research projects and studies by Katholieke Universiteit Leuven, St George's, University of London and Universitat Politècnica de València.

- C2 - Design and implementation of a Single-Voxel (SV) 1H-MRS semi-automatic pre-processing pipeline for CURIAM-BT CDSS. The definition of a standard pipeline ensures that the new observations will be processed following the same procedures as the ones used to train the classifiers included in the CDSS and it helps to improve the reproducibility of the results obtained. CURIAM-BT CDSS and this pipeline are registered by the Universitat Politècnica de València, it has been presented in different congresses $\left[\mathrm{SGGV}^{+} 09, \mathrm{SGGV}^{+}\right.$08], and it is being evaluated at the Hospital Universitario Doctor Peset, Hospital Quirón València and Hospital de la Ribera.

- C3 - Design and implementation of Arrhythlab: A software for ECG automatic pre-processing, quality control, segmentation, and beat labeling. This software is designed to facilitate the compilation of annotated ECG datasets for arrhythmia studies in which every beat needs to be labeled. Arrhythlab has already allowed the compilation of a large annotated ECG dataset throughout the Sistema Experto en Electrocardiografía project (TSI-020302-2010-136). Arrhythlab is 
being registered by the Universitat Politècnica de València, and it is freely downloadable for its use in research studies.

- C4 - Evaluation of the compatibility between two coexisting MRS scanner technologies using 1.5T and 3T magnets. This study demonstrates that 3T SV-MRS data can be used with the currently available automatic brain tumour diagnostic classifiers trained on datasets of $1.5 \mathrm{~T}$ spectra. The results obtained are published as a journal paper in $\left[\mathrm{FGNV}^{+} 11\right]$.

- C5 - Design of Functional Data Analysis based on Region Segmentation (RSFDA) algorithm for extracting discriminant MRS functional features of brain tumours. This algorithm takes into account the no independent and identically distributed (i.i.d.) properties of the signals descriptors, and extract independent features to improve the robustness of the signal classification. The RSFDA algorithm has been submitted for publication.

- C6 - Improvement of PET image quality by the introduction of new Machine Learning (ML) based algorithms for the reduction of random coincidences. The resolution and signal-to-noise ratio obtained in PET systems depends directly on the ability to remove or correct accidental coincidences among others. The improvement on the reduction of random coincidences algorithms affects directly to the acquisition time or the dose of injected radio-nuclei needed. This novel technique was applied in the challenging case of preclinical small animal PET scanners. The results obtained were presented in different international congresses and published as proceedings in [FGOTR08, $\mathrm{FGOC}^{+} 10 \mathrm{a}$, $\left.\mathrm{FGOC}^{+} 10 \mathrm{~b}\right]$.

- C7 - Multicenter evaluation of automated arrhythmia detection and classification models using 12-channel ECG. A part of this study, focused on the identification of significant points for the study of arrhythmias in electrocardiograms has been presented in $\left[\mathrm{FGAG}^{+} 11\right]$.

In addition, the author of this thesis has collaborated as co-author in different papers related to the design of classification models for the support in the diagnosis of brain tumours by MRS signals. These include: (1) the design of incremental learning algorithms for MRS data, (2) the multiprojectmulticenter evaluation of automated brain tumour classifiers using singlevoxel MRS, (3) the development of classifiers for the three most prevalent tumour types in children, and (4) the design of a novel method for the classification of MRS signals using LCModel [Pro01]. The results of these studies were accepted as journal papers in $\left[\mathrm{TFGV}^{+} 11, \mathrm{GGLJS}^{+} 09\right.$, RFGOH12] and presented in different international congresses [GGLJS ${ }^{+} 08$, $\mathrm{VGGT}^{+} 09, \mathrm{GGEJS}^{+} 09, \mathrm{VTFG}^{+}$12]. Collaboration on the preparation of the datasets, signal processing, the writing and experimental design was carried out in these works. 


\subsection{PROJECTS AND PARTNERS}

Throughout the development of this thesis, the author, has been actively involved in the following projects with the collaboration of clinical, scientific and private sector partners:

Etumour [The07] Web accesible (Nuclear) Magnetic Resonance (MR) decision support system for brain tumour diagnosis and prognosis, incorporating in vivo and ex vivo genomic and metabolomic data. European Commission (VI Framework Program, LSHC-CT-2004-503094, 2004-2009).

Objectives: (1) Development of a web-accessible CDSS that has a Graphical User Interface (GUI) to display clinical, metabolomic and genetic brain tumor data. (2) Facilitation of evidence-based clinical decision-making (e.g. diagnosis, prognosis, optimal treatment strategies etc.) by using statistical pattern recognition analysis of molecular images of brain tumours (using MRS) and incorporating new criteria such as genetic based tumour classifications and related clinical information such as patient outcome.

Partners: University of Valencia (Valencia, Spain), Universitat Autònoma de Barcelona (Barcelona, Spain), St George's Hospital Medical School (London, UK), University Medical Center Nijmegen (Nijmegen, Netherlands), Stichting Katholieke Universiteit (Nijmegen, Netherlands), Université Joseph Fourier U594 (Grenoble, France), MicroArt S.L. (Barcelona, Spain), Hospital San Joan de Deu (Esplugues de Llobregat, Spain), Pharma Quality Europe, s.r.l. (Barcelona, Spain), Hyperphar Group SpA. (Milan, Italy), Katholieke Universiteit Leuven (Leuven, Belgium), Siemens AG, Medical Solutions (Erlangen, Germany), SCITO, S.A (Grenoble, France), Deutsche Krebsforschungs zentrum Heidelberg (Heidelberg, Germany), Bruker Biospin SA. (Wissembourg, France), Institute of Child Health - University of Birmingham ( Birmingham, United Kingdom), INSERM U318 (Grenoble, France), Fundación para la Lucha contra Enfermedades Neurológicas de la Infancia (Buenos Aires, Argentina), Medical University Lodz (Lodz, Poland) and IBIME-ITACA group from Universitat Politècnica de València (Valencia, Spain).

healthagents [GVMJS+ 07] Agent-based distributed decision support system for brain tumour diagnosis and prognosis. European Commission (VI Framework Program, IST-2004-27214, 2006-2009).

Objectives: To create a distributed Data Warehouse (dDWH) with the world's largest network of interconnected databases of clinical, histological, and molecular phenotype data of brain tumour patients. The $\mathrm{dDWH}$ will facilitate evidence-based clinical decision-making using MR and genetic based tumour classifications and will include new criteria from the automated analysis of each local database. 
Partners: University of Valencia (Valencia, Spain), MicroArt S.L. (Barcelona, Spain), Universitat Autònoma de Barcelona (Barcelona, Spain), Pharma Quality Europe, s.r.l. (Barcelona, Spain), Katholieke Universiteit Leuven (Leuven, Belgium), University of Birmingham (Birmingham, UK), University of Edinburgh (Edinburg, UK), University of Southampton (Southampton, UK), and IBIME-ITACA group from Universitat Politècnica de València (Valencia, Spain)

D S S P R S TATA Sistema de Soporte a la Decisión para Detección Precoz de Cáncer de Próstata. Ministerio de Industria Turismo y Comercio de España (PROFIT FIT-340001-2007-14, 2007-2009).

Objectives: Development of a CDSS for the support in the prostate tumour diagnosis based on MRSI data.

Partners: MicroArt S.L. (Barcelona, Spain), CRC-Hospital Universitari Sagrat Cor (Barcelona, Spain), CRC-IAT Centre d'Imatge Molecular Barcelona (Barcelona, Spain), and IBIME-ITACA group from Universitat Politècnica de València (Valencia, Spain).

DSSPROSTATA2 Sistema para el diagnóstico precoz de tumores de próstata. Ministerio de Industria Turismo y Comercio de España (AVANZA, TSI-020302-2009-65, 2009-2010).

Objectives: Development of a CDSS for the support in the prostate tumour diagnosis based on MRSI data.

Partners: MicroArt S.L. (Barcelona, Spain), CRC-Hospital Universitari Sagrat Cor (Barcelona, Spain), CRC-IAT Centre d'Imatge Molecular (Barcelona, Spain), and IBIME-ITACA group from Universitat Politècnica de València (Valencia, Spain).

SEE Sistema Experto en Electrocardiografía. Ministerio de Industria, Turismo y Comercio (AVANZA I+D, TSI-020302-2010-136, 2010-2011).

Objectives: Development of a collaborative platform for the offshoring of clinical decisions in the context of cardiac diseases.

Partners: GEM-MED S.L. (Barcelona, Spain), Masmovil S.A (Spain), ISoco S.A. (Spain), Hospital Universitario de La Ribera (Alzira, Spain), Ascamm (Spain), Centro Tecnológico Universidad de Málaga (Málaga, Spain), Consultores Bayés (Vic, Spain), Fundación Iavante (Spain), and IBIME-ITACA group from Universitat Politècnica de València (Valencia, Spain).

Microcluster Desde de la vanguardia de la Física de Partículas, Nuclear y Astropartículas hasta la trasferencia en Computación Distribuida, Imagen y Aceleradores en Medicina y Tecnologías Marinas. Research microclusters (VLC/CAMPUS, 2011). 
Objectives: (1) Fundamental, theoretical, and experimental research, in particle, astroparticles and nuclear physics. (2) Applications of particle and nuclear physics in medical physics: image and accelerators for health (consolidation of Singular Infrastructure for Medical Physics: IFIMED). (3) Applications of particle physics in distributed computing: GRID and eScience (4) Applications of physics to marine technology. (5) Consolidation of the national center for particle, astroparticles and nuclear physics (CPAN).

Partners: UVEG-CSIC Instituto de Fisica Corpuscular (Valencia, España), UPV Departamento de Física Aplicada (Valencia, Spain), UVEG Instituto Ciencia Materiales (Valencia, Spain), Hospital Universitari La Fe (Valencia, Spain), Serveis de'Oncologia Radioteràpica (Valencia, Spain), Institut Valéncia d'Oncologia (Valencia, Spain), and IBIME-ITACA group from Universitat Politècnica de València (Valencia, Spain).

The author of this thesis performed a research intership at Division of Head and Vascular Sciences of the St George's Hospital Medical School - University of London (London, UK), from $27^{\text {th }}$ October 2009 to $27^{\text {th }}$ January 2010. The research stay was conducted under the general direction of the Head of Division, Professor John Camm, and under the specific direction of Dr. Franklyn A. Howe.

\subsection{CONTENTS}

This thesis is divided in three main parts: The first one is an introduction to the thesis and its theoretical basis; the second part includes the main scientific contributions of this thesis; and finally the conclusions of the thesis will be the third part.

The first part consists on two chapters. Chapter 1 INTRODUCTION, presents the motivation, the goals, and the contributions of the thesis. The second one, 2 BIOMEDICAL SIGNALS AND THE CLASSIFICATION PROBLEM, introduces the theoretical framework concerning the biomedical signals and their classification.

As mentioned, the second part of this thesis contains the main contributions to different elements of a biomedical signal supervised classification system: Automatic pre-process and compatibility of biomedical signals, feature selection/extraction of biomedical signals, and development of classification models. These contributions are organized in five chapters. Chapter 3 BIOMEDICAL SIGNAL PROCESSING AND VISUALIZATION, includes three contributions related to the design and implementation of automatic pre-processing modules for MRSI, MRS and ECG signals. In chapter 4 COMPATIBILITY BETWEEN $3 T$ AND 1.5T SV-MRS IN CLASSIFICATION PROBLEMS, the compatibility between $3 \mathrm{~T}{ }^{1} \mathrm{H}$ SV-MRS data and automatic brain tumour diagnosis support systems based on $1.5 \mathrm{~T}{ }^{1} \mathrm{H}$ SV-MRS, has been analyzed. The results obtained in this chapter demonstrate that 3T SV-MRS data can be used with the currently available automatic brain 
tumour diagnostic classifiers. A new algorithm, RSFDA, for extracting discriminant MRS functional features of brain tumors is presented in chapter 5 EXTRACTING MRS DISCRIMINANT FUNCTIONAL FEATURES. An exhaustive characterization of the method is described in this chapter using controlled and real scenarios, and the performance of RSFDA has been compared with other widely used feature extraction methods. In Chapter 6 REDUCTION OF RANDOM COINCIDENCES IN PET, a supervised classifier based on Artificial Neural Networks (ANN) has been designed to address the problem of reducing random coincidences in small animal PET. Finally, chapter 7 AUTOMATIC CLASSIFICATION OF ARRHYTHMIAS $B A S E D O N E C G$, presents a multicenter study for the assessment of different feature extraction methods, classification algorithms, and aligning methods for arrhythmia automatic detection and classification by ECG signals.

The last part includes the conclusions obtained in the thesis, organized in a single chapter, 8 CONCLUDING REMARKS AND FUTURE WORK.

In the schema presented on Figure 1 the distribution of chapters in each part or thematic block is shown. Moreover, it is also shown the relation between the thematic blocks and the different contributions (papers and congresses) and projects related to the objectives of this work, in which the author of this thesis has participated. 
PARTS/BLOCKS

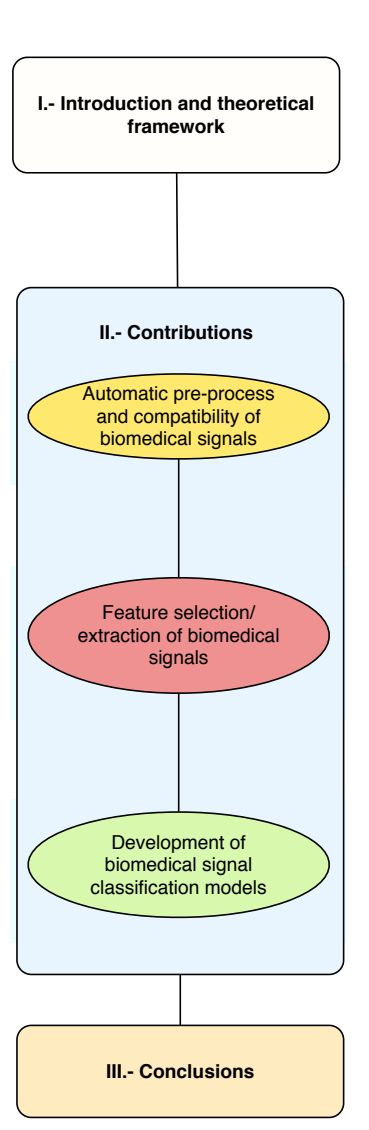

CHAPTERS

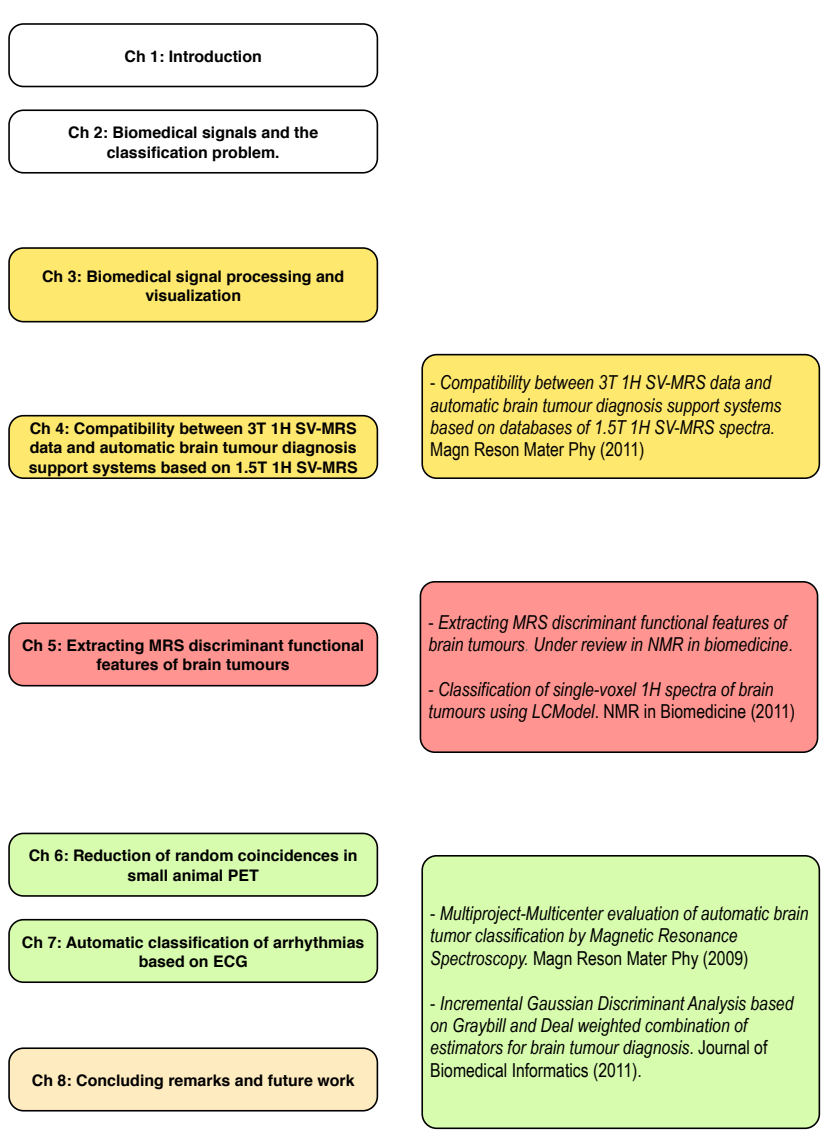

CONGRESSES

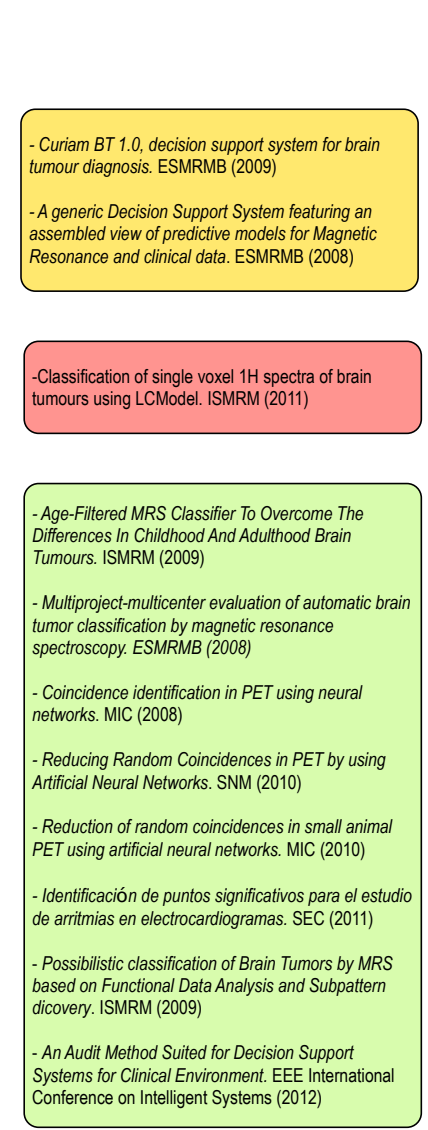

PROJECTS

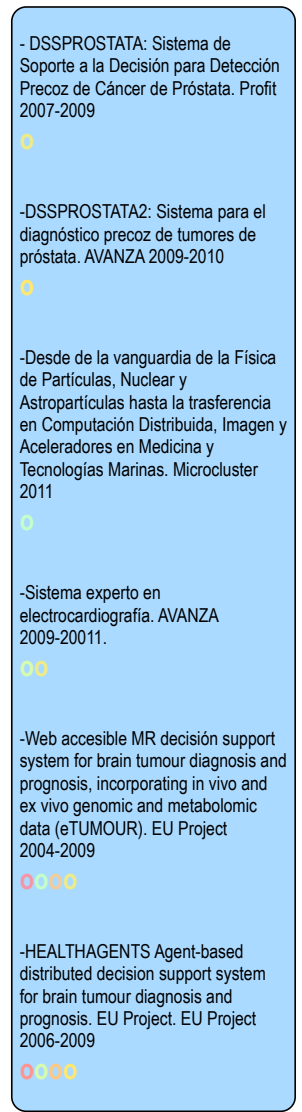

REGISTERED SOFTWARE

Jystem for Becisison Support

Arythlab: Beat labeling software for

arrhyhmia studies.

MRSILab: MRSI procesing and

visualization toolbox.

\section{RESEARCH STAYS \\ Theme: Clinical decision support \\ systems based on MRSS for brain and \\ Place: St. George's, University of \\ London, London, UK. \\ Length: 3 months \\ Year: 2009-2010.}

Figure 1: Summary of the thesis including contributions, collaboration in scientific projects and research interships. 


PROBLEM

\subsection{INTRODUCTION}

Biomedical signals are those used in biomedical fields, mainly for extracting information on a biologic system under investigation [Coh00]. Biomedical signals originate from a variety of sources as can be: electrical, impedance, acoustical, magnetic, mechanical, chemical or optical among others.

The classification of biomedical signals requires a deep knowledge of how these signals are generated and acquired. Moreover, since the signals are complex data, it is mandatory to know how to preprocess them and how to extract their most relevant features. This knowledge allows the interpretation of the information contained in the signals and the evaluation of the obtained results.

Moreover, the classification of biomedical signals, requires to know how to analyze and transform the signal points in order to reduce and optimize the inputs of classification algorithm. In this sense, the deep understanding of Feature Extraction (FE) algorithms becomes necessary.

To introduce the theoretical framework concerning the biomedical signals and their classification, this chapter is divided into two main parts. In the first part (sections $2.2,2.3,2.4$ ), an introduction to the physical origin and reconstruction of the biomedical signal included in this thesis (MRS and MRSI, ECG, and PET) is presented. The second part of this chapter (2.5) is focused on the methods and algorithms involved in the design, implementation, and evaluation of a classification model (i.e. FE methods, classification algorithms, and evaluation strategies).

\subsection{NUCLEAR MAGNETIC RESONANCE SPECTROSCOPY}

\subsubsection{The nucleus under a magnetic field}

The NMR is based on the spin angular momentum property $\mathbf{I}$ of the nuclei. The fundamental postulates of quantum mechanics establish that the angular momentum of elementary particles are limited to discrete values. More specifically, the amplitude of the angular momentum $\mathbf{L}$ is defined by 


$$
\mathrm{L}=\frac{\mathrm{h}}{2 \pi} \sqrt{\mathrm{I}(\mathrm{I}+1)}
$$

where $h$ is the Plank's constant, and I is the spin quantum number which can only be integer or half integer. In quantum mechanics the direction of the angular momentum $\mathbf{L}$ is also discretized, and the component of $\mathbf{L}$ in a given direction $z$, is given by

$$
\mathrm{L}_{z}=\frac{\mathrm{h}}{2 \pi} \mathrm{m}
$$

where only $2 \mathrm{I}+1$ values are allowed for $m(m=I, I-1, I-2, \ldots,-I)$.

Nuclei with an odd number of protons or neutrons (as ${ }^{31} \mathrm{P},{ }^{19} \mathrm{~F},{ }^{13} \mathrm{C}$ or ${ }^{1} \mathrm{H}$ ) have a non-zero spin. A non-zero spin nuclei has an associated intrinsic magnetic moment $\mu$ defined by

$$
\boldsymbol{\mu}=\gamma \mathbf{L}
$$

where $\gamma$ is the gyromagnetic constant proportional to the charge-to-mass ratio of the nucleus, and $\mu$ and $L$ are vector quantities.

The component of the magnetic moment along a given direction $z, \mu_{z}$, is quantized due to $L_{z}$ is quantized too. From Equations 2 and 3 we obtain

$$
\mu_{z}=\gamma \frac{h}{2 \pi} m
$$

If a nucleus with a magnetic momentum $\mu$ and an angular momentum $\mathbf{L}$ is under the influence of a magnetic field $\mathbf{B}_{\mathbf{o}}$ in the $z$ direction, the particle acquires a magnetic energy given by

$$
E_{p o t}=-\mu_{z} \mathbf{B}_{\mathbf{o}}=-\gamma \frac{h}{2 \pi} m \mathbf{B}_{\mathbf{o}}
$$

In simplest case of ${ }^{1} \mathrm{H}$ (the most commonly used nuclei in NMR), $\mathrm{I}_{\mathrm{H}}=1 / 2$ and therefore only $m=\{\langle 1 / 2\rangle,\langle-1 / 2\rangle\}$ values are allowed depending if the spin is aligned or opposite to the direction of $\mathbf{B}_{\mathbf{0}}$. In this case each of the spin stated defined by $m$ will have different energies associated. The energy difference between both directions (see Figure 2) is given by

$$
\Delta \mathrm{E}_{\mathrm{pot}}=\left|\mathrm{E}_{\mathrm{pot}}<\frac{1}{2}>-\mathrm{E}_{\mathrm{pot}}<-\frac{1}{2}>\right|=\gamma \frac{\mathrm{h}}{2 \pi} \mathrm{m} \mathbf{B}_{\mathbf{0}}
$$

At equilibrium, the relative populations of the states are determined by the Boltzman's law. In a system of spin $1 / 2$ nuclei (as can be ${ }^{1} \mathrm{H}$ ), the relative populations between the two allowed states are:

$$
\frac{\mathrm{N}_{\downarrow}}{\mathrm{N}_{\uparrow}}=\exp \left(-\frac{\Delta \mathrm{E}_{\text {pot }}}{\mathrm{kT}}\right) \approx 1-\frac{\Delta \mathrm{E}_{\mathrm{pot}}}{\mathrm{kT}} \approx 1-\frac{\gamma \mathbf{B}_{\mathbf{0}}}{\mathrm{kT}}
$$




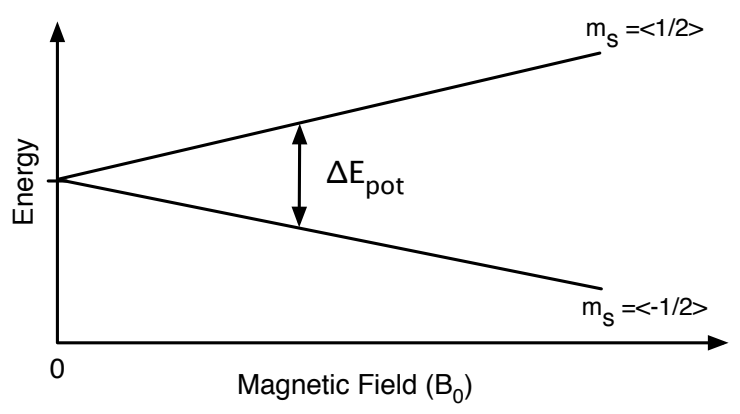

Figure 2: Difference of potential energy between the levels $<-1 / 2>$ and $<1 / 2>$.

where $k T$ represents the thermal energy, $k$ is the Boltzman's constant, and T the absolute temperature of the sample in Kelvin. The approximation done in Equation 7 assumes $\Delta \mathrm{E}_{\text {pot }} \ll \mathrm{kT}$ (even for the high magnetic fields used in NMR).

NMR technique uses the transition between these levels to detect and quantify nuclei. Since the $\Delta \mathrm{E}_{\mathrm{pot}}$ is in the range of radio frequency radiation, it is possible to realign or flip the nuclear spins in the higher-energy direction, by the stimulation of the sample by Radio Frequency (RF) radiation with an energy close to $\Delta \mathrm{E}_{\text {pot }}$.

Since the energy of an electromagnetic wave is given by $E=h v$, the frequency $\nu_{\mathrm{L}}$, associated with the energy gap $\Delta \mathrm{E}_{\text {pot }}$ is given by

$$
v_{\mathrm{L}}=\frac{\gamma}{2 \pi} \mathrm{B}_{0}
$$

After absorbing the energy of these pulses, nuclei will trend to return to the lower-energy state reemitting RF radiation according, providing this way, information of the nuclei concentration and about the properties of their magnetic environment (chemical composition of the sample).

Although only quantum mechanics can completely explain the behavior of a nucleus under a magnetic field, some of this features can be explained by the classical theory of electromagnetism, giving us a more graphical interpretation of the phenomena. From a classical description, the motion of a magnetic moment $\boldsymbol{\mu}$ under the influence of a external magnetic field $\mathbf{B}_{\mathbf{0}}$, is described by

$$
\frac{\mathrm{d} \boldsymbol{\mu}}{\mathrm{dt}}=\gamma \boldsymbol{\mu} \times \mathbf{B}_{\mathbf{o}}
$$

when $\mathbf{B}_{\mathbf{0}}$ is a static magnetic field in the $z$ direction, the solution of this equation is give by

$$
\mu_{x}(t)=\mu_{x}(0) \cos \omega_{L} t+\mu_{y}(0) \sin \omega_{L} t
$$




$$
\begin{gathered}
\mu_{y}(t)=\mu_{y}(0) \cos \omega_{L} t-\mu_{x}(0) \sin \omega_{L} t \\
\mu_{z}(t)=\mu_{z}(0)
\end{gathered}
$$

where $\omega_{\mathrm{L}}=\gamma \mathbf{B}_{\mathbf{0}}$.

According to Equations 10, 11, and 12, $\boldsymbol{\mu}$ precesses about the magnetic field direction $\mathbf{B}_{\mathbf{0}}$ with an angular frequency $\omega_{\mathrm{L}}$ known as the Larmor's frequency. Notice that $\omega_{\mathrm{L}}$ is the angular frequency associated to $v_{\mathrm{L}}$, that is $\omega_{\mathrm{L}}=2 \pi v_{\mathrm{L}}$.

\subsubsection{Excitation and signal generation}

\section{Nuclear magnetization}

Although the magnetic field of a nucleus is complex, we can simplify the net magnetic field in a given volume as the vector sum of their magnetic moments, or nuclear magnetization $\mathbf{M}$. If the precession of nuclei magnetic moments are phased, the transverse component of $\mathbf{M}$ (perpendicular to $\left.\mathbf{B}_{\mathbf{0}}\right), M_{x y}$, rotates at the Larmor's frequency $v_{L}$, producing an oscillating magnetic field, generating a variable magnetic flux in the RF receiver coil, and therefore inducing in this way a small alternating voltage on it. However at equilibrium, the nuclei are not phased, the transverse components of their magnetic moments cancel-out, and no signal is detected.

The longitudinal component of $\mathbf{M}$ (parallel to $\mathbf{B}_{\mathbf{0}}$ ) depends on the proportion nuclei magnetic moments aligned with the static field $\mathbf{B}_{\mathbf{0}}$. This component, however cannot be detected because it does not oscillate and therefore does not generate induced signals in the coils.

\section{RF Excitation}

In order to obtain a signal in the receiver coil, we must phase the nuclei magnetic moments perturbing the system from its equilibrium state. To do so, a secondary oscillating magnetic field $B_{1}$ produced by a RF pulse with a frequency equal to Larmor's frequency is applied. This RF pulse allows the nuclei to precess in synchrony, and therefore, to generate a transverse component of $\mathbf{M}$. Moreover, as the $B_{1}(t)$ field transfer energy to the nuclei, some of the nuclei promote to the higher energy level and the longitudinal magnetization gradually decrease (see Figure 3). The use of Larmor's frequency ensures the maximum energy absorption from the pulse by the nuclei, due to the coincidence between the photon energy and the energy gap $\Delta \mathrm{E}_{\text {pot }}$ (resonance phenomena).

The excitation is quantified by the flip angle $\alpha$ though which the net magnetization is tipped away from the longitudinal axis. The flip angle $\alpha$ is proportional to $B_{1}$ and pulse duration. 


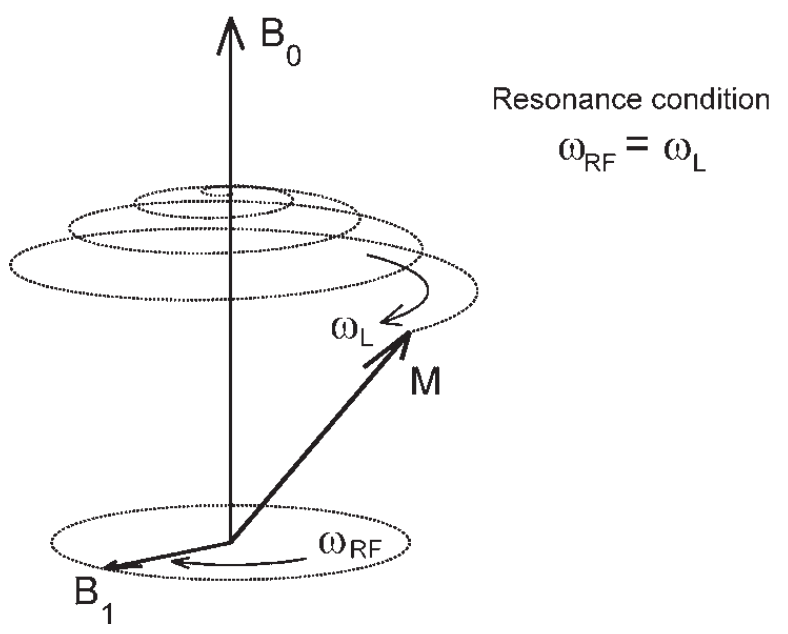

Figure 3: The RF pulse at the Larmor's frequency $\left(B_{1}\right)$, causes the magnetization vector $\mathbf{M}$ having a transverse component. Extracted from [Pra06] with kind permission from Springer Science and Business Media.

\section{Signal generation and spin relaxation}

After the RF excitation, the net magnetization vector $\mathbf{M}$ is perturbed out of the equilibrium, and $\mathbf{M}$ is displaced from the $\mathbf{B}_{\mathbf{0}}$ axis ( $z$ axis). Because $\mathbf{M}$ is no longer aligned to $\mathbf{B}_{\mathbf{0}}$, it experiences a torque due to $B_{0}$, a precession movement at the Larmor's frequency begins, and it generate a induced current into the coils. As was already mentioned, the amplitude of the alternating voltage induced in the coil is proportional to the transverse magnetization component $M_{x y}$.

In the ideal scenario in which no energy loss or loss of phase coherence occurs, the signal will be a sinusoid at the Larmor's frequency. However, this scenario is not realistic. Loss of phase coherence occurs due to internuclear and inter molecular forces, and energy loss to their surroundings (nuclei return to low energy levels) is produced. These processes are termed transverse relaxation and longitudinal relaxation respectively. Although these relaxation processes limit the acquisition time, it is possible exploit these phenomena to obtain information about the sample analyzed. Because their relaxation rates depend on the molecular environment of the nuclei, it is possible to obtain different contrast among different tissues in Magnetic Resonance Imaging (MRI).

\section{- Longitudinal relaxation: $T_{1}$ (Loss of energy)}

After an $\alpha$-degree RF pulse, the recovery of the longitudinal magnetization in the direction of $\mathbf{B}_{\mathbf{0}}$ (loss of energy) follow,

$$
M_{z}(t)=M_{0}\left(1-(1-\cos \alpha) e^{\left(-t / T_{1}\right)}\right)
$$


where $M_{0}$ corresponds to $\mathbf{M}$ in the equilibrium. The $T_{1}$ parameter characterizes the longitudinal relaxation and is defined as the time interval needed for the longitudinal magnetization to recover a value of $63.2 \%$ of the equilibrium value $\mathbf{M}_{\mathbf{0}}$. The value of $T_{1}$ varies significantly between tissues, according to their molecular structure.

- Transverse relaxation: $T_{2}$ and $T_{2}^{*}$ (Loss of phase coherence)

Transverse relaxation occurs faster than longitudinal relaxation. It includes several mechanisms that result in a loss of phase coherence. On the one hand, there are microscopic effects that depend on intrinsic factors such as molecular tissue type or molecular size, that are represented by the $T_{2}$ value. $T_{2}$ value represents the time interval required for the transverse magnetization to decay to $36.7 \%$ of its initial value $\mathbf{M}_{\mathbf{0}}$. The $T_{2}$ decay of the transverse magnetization $M_{x y}$ following a $\alpha$-degree RF pulse could be expressed as:

$$
M_{x y}(t)=M_{0} \sin \alpha e^{i\left(\omega_{l} t+\varphi\right)} e^{-t / T_{2}}
$$

On the other hand, effects such as magnetic field inhomogeneities also contribute to the loss of phase coherence. In order to include this effect another relaxation time $T_{2}^{*}$ is defined. $T_{2}^{*}$, describes the observed rate of decay of the transverse magnetization:

$$
\frac{1}{\mathrm{~T}_{2}^{*}}=\frac{1}{\mathrm{~T}_{2}}+\frac{1}{\mathrm{~T}_{2}^{\prime}}
$$

where $T_{2}^{\prime}$ describes decay in signal due to magnetic field inhomogeneity:

$$
\frac{1}{\mathrm{~T}_{2}^{\prime}}=\gamma \Delta \mathrm{B}_{0}
$$

$T_{2}^{*}$ parameter is usually much smaller than $T_{2}$.

Decoherence because of inhomogeneity is dependent on the location of the molecule in the magnet and for molecules that are not moving, the signal can be recovered by performing a spin echo experiment.

\subsubsection{The free induction decay and the spin-echo}

The electrical signal generated in receiver coils after the RF pulse excitation is known as the Free Induction Decay (FID) (see Figure 4) The FID occurs on a timescale $T_{2}^{*}$, and represents the value of $M_{x y}$ after RF pulse.

$$
M_{x y}(t)=M_{0} \sin \alpha e^{i\left(\omega_{1} t+\varphi\right)} e^{-t / T_{2}^{*}}
$$

The de-phasing due to field inhomogeneities can be reversed using a simple refocusing procedure. It consist on the use of a $180^{\circ}$ RF pulse (refocusing 


\section{Relaxation}

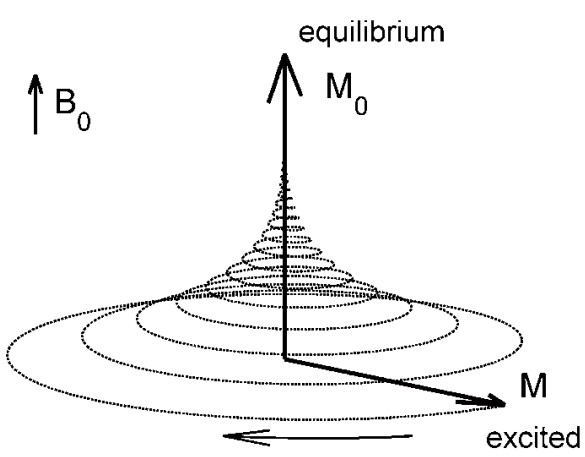

$\omega_{\llcorner}$

\section{Free induction decay}

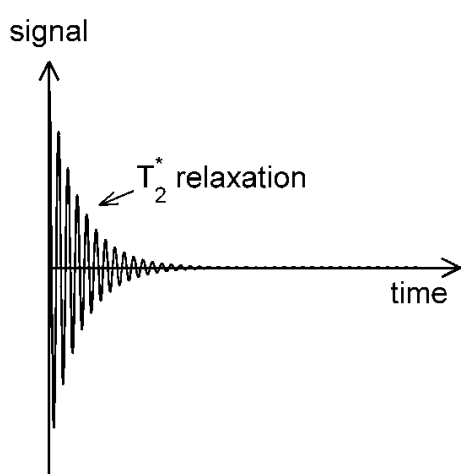

Figure 4: Relaxation of the magnetization vector $\mathbf{M}$ after the RF pulse (left), and signal generated in the coil (FID) by the transverse component of $M$ (rigth). Extracted from [Pra06] with kind permission from Springer Science and Business Media.

pulse) to reverse the phase differences accumulated since the first $90^{\circ} \mathrm{RF}$ pulse (excitation pulse), as can be seen in Figure 5. The time taken for re-phase equals the time during they are allowed to de-phasing, and the total time taken is known as echo time, Echo Time (TE). It is important to notice that this procedure could not correct loss of phase coherence due to microscopic effects that depends on intrinsic factors such as molecular tissue type or molecular size (T2), as can be seen in Figure 5. The time between two excitation pulses $\left(90^{\circ} \mathrm{RF}\right.$ pulses) is known as repetition time (Repetition Time (TR)).

\subsubsection{Spatial localization of magnetic resonance signals}

The use of spatial localization techniques is needed in order to select the location from where we want to obtain NMR information. There are three main methods of spatial localization that are combined in the pulse sequence depending on the imaging modality: slice selection, frequency encoding and phase encoding. All these spatial localization techniques are based on the use of magnetic field gradients.

The sequence diagrams are used to describe the implementation of particular NMR sequences in time, including the spacial localization sequences. In Figure 6 a basic acquisition for MRI is presented 


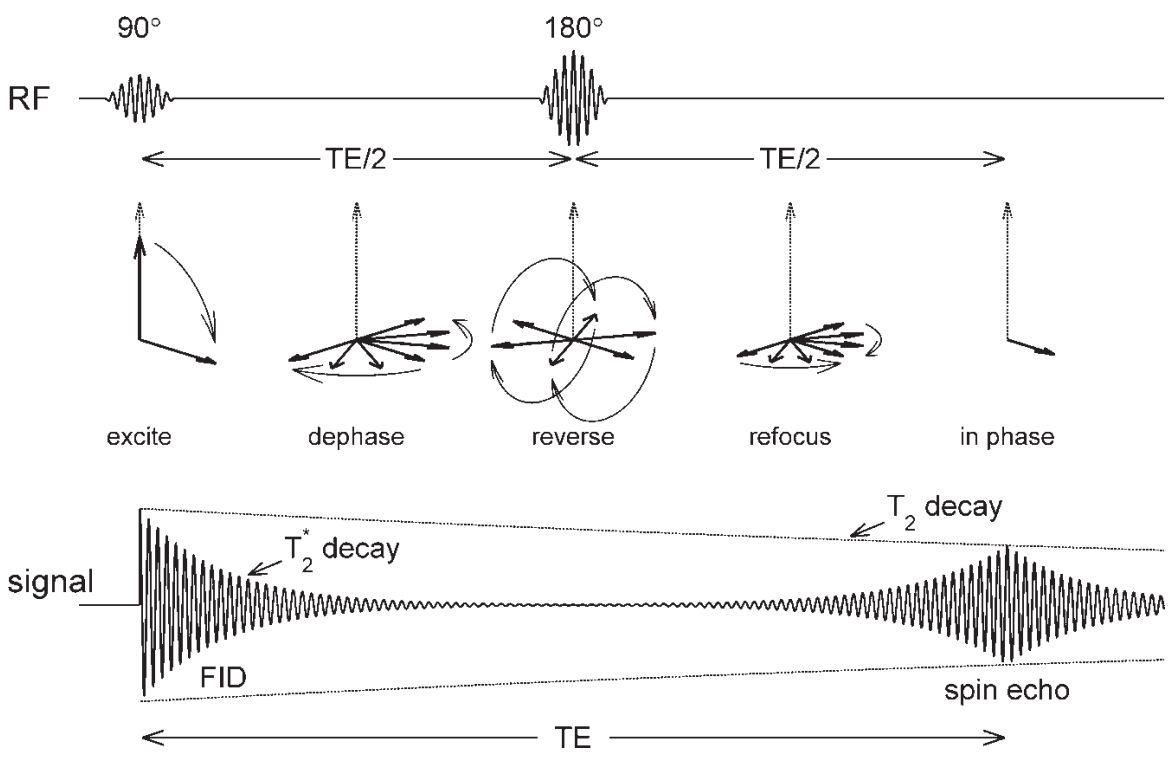

Figure 5: Schema of the spin-echo sequence (top), the behavior of the magnetization vectors (middle), and the associated signal generated in the coil (bottom). Extracted from [Pra06] with kind permission from Springer Science and Business Media.

\section{Magnetic field gradients}

A magnetic field gradient is an additional magnetic field in the same direction of $\mathrm{B}_{0}$, but whose amplitude varies linearly with a specific axis. The gradients along the three axis are defined as:

$$
\begin{aligned}
\mathrm{G}_{x} & =\mathrm{dB}_{z} / \mathrm{d} x \\
\mathrm{G}_{\mathrm{y}} & =\mathrm{dB}_{z} / \mathrm{d} y \\
\mathrm{G}_{z} & =\mathrm{dB}_{z} / \mathrm{d} z
\end{aligned}
$$

These gradients modify the magnetic field, in such a way that every point of the sample gets assigned to a specific value of the magnetic field, and therefore to a specific Larmor frequencies.

\section{Slice selection}

The slice selection technique is based on the application during the excitation RF pulse of a gradient in the perpendicular direction to the desired slice. In this way each plane in the gradient direction (for example $z$ ) will be affected by a specific value of magnetic field $B(z)=\mathbf{B}_{\mathbf{0}}+z \mathrm{G}_{z}$. The effect of this gradient is to make the resonance frequency a function of position along the gradient direction (see Figure 7): 


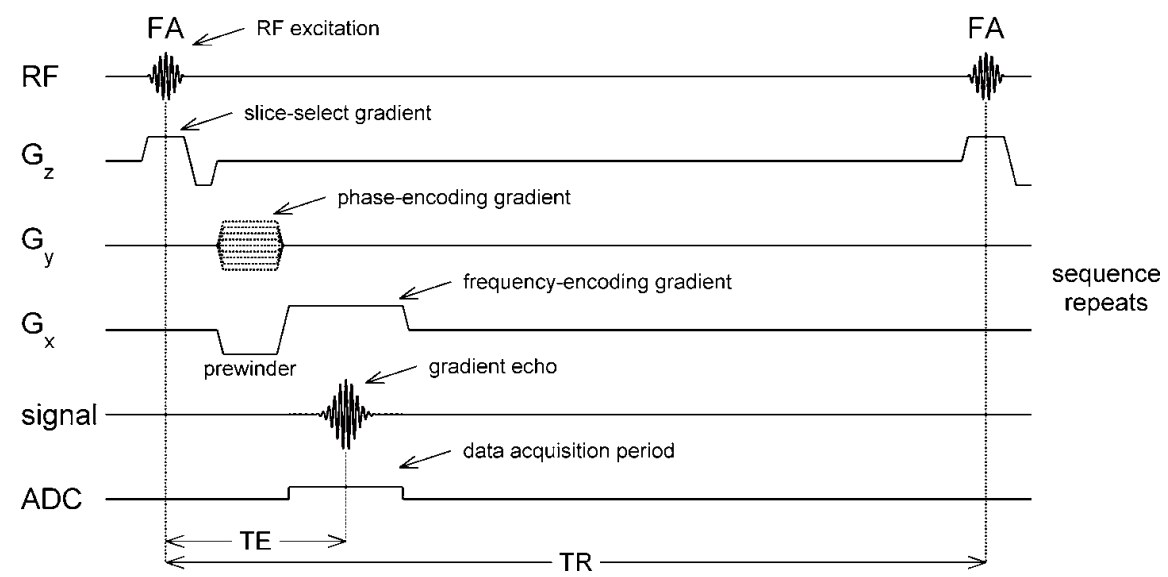

Figure 6: Basic sequence for MRI acquisition, including: slice selection, frequency encoding and phase encoding localization methods. Extracted from [Pra06] with kind permission from Springer Science and Business Media.

$$
\omega(z)=\gamma B_{z}(z)=\gamma\left(B_{0}+z G_{z}\right)
$$

Since the RF pulse cannot be a single frequency pulse, and also contains a narrow spread of frequencies close to the fundamental resonance frequency of the magnet, the width of the slice is given by:

$$
\delta(z)=\delta \omega / \gamma \mathrm{G}_{z}
$$

where $\delta \omega$ is the bandwidth of the frequencies contained in the pulse.

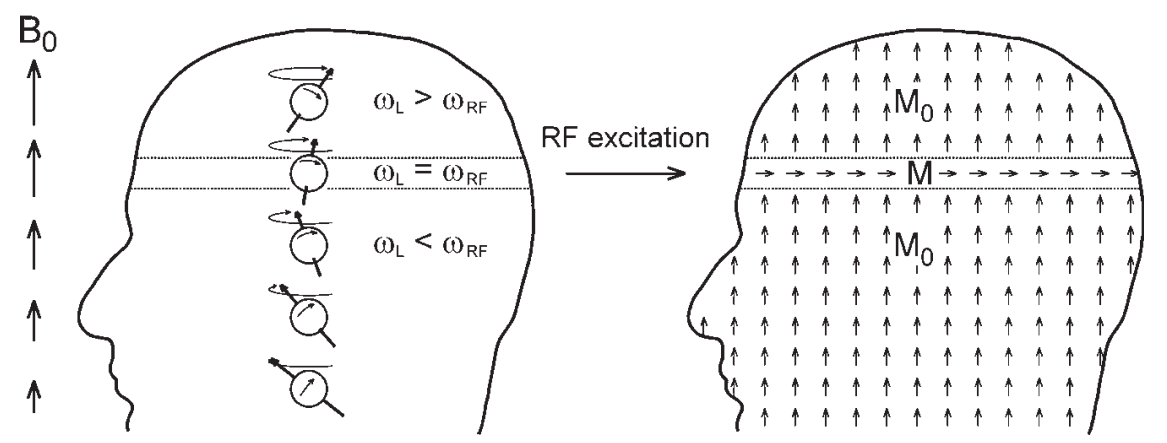

Figure 7: Slice selection mechanism. The gradient in $z$ make the resonance frequency a function of position along $z$. The slice selection gradient is applied during the RF excitation. Extracted from [Pra06] with kind permission from Springer Science and Business Media. 


\section{Frequency encoding}

The frequency encoding technique is based on making the oscillation frequency in a NMR signal linearly dependent on its spatial origin. If we apply a gradient in a $x$ direction, we make the resonance frequency a function of position along $x$ (the gradient direction). In the frequency encoding technique, the gradient is applied after RF pulse and therefore is applied when the sample is already excited (unlike the slice selection technique). By this way, different frequencies in the obtained FID signal corresponds to different positions of the sample in the direction of the gradient (see Figure 8).

That is, if we consider a idealized one-dimensional sample with spin distribution $\rho(x)$, the FID signal $d s(x, t)$ generated locally from spins in a infinitesimal interval $d x$ at point $x$ (without considering $T_{2}$ effect), is:

$$
d s(x, t)=c \rho(x) e^{-i \gamma\left(B_{0}+x G x\right) t} d x
$$

where $\mathrm{c}$ is a proportionality constant. As a consequence the signal obtained from the entire sample when the gradient is applied is:

$$
\begin{array}{r}
s(t)=\int_{-\infty}^{\infty} d s(x t)=\int_{-\infty}^{\infty} c \rho(x) e^{-i \gamma\left(B_{0}+x G x\right) t} d x \\
=\left[\int_{-\infty}^{\infty} c \rho(x) e^{-i \gamma x G x t} d x\right] e^{-i \omega_{0} t}
\end{array}
$$

where, after removing carrier signal $e^{-i \omega_{0} t}$ (demodulation), is easy to see that each different position in the sample has an associated frequency in the FID signal. Generalizing for the case of the frequency encoding direction $\mathbf{r}$,

$$
s(t)=c \int_{-\infty}^{\infty} \rho(\mathbf{r}) e^{-i \gamma r G_{f r e q} t} d r
$$

\section{Phase encoding}

In the phase encoding technique a gradient pulse is applied in the phaseencoding direction before signal acquisition. This gradient pulse creates a dependence on the position of the Larmor frequency of the spins, but only for a brief period, resulting in a relative phase shift among the spins. However, because the definition of phase, a spin affected by a phase shift $\delta$ and a spin affected by $n 2 \pi \delta$ where $n$ is an integer, will have the same phase. To solve this ambiguity, the entire process of excitation and signal acquisition must be repeated many times, by using gradient pulses of incrementally different strengths. This way, we have uniquely identified the position of the sample along the direction of the gradient (see Figure 8).

Considering a unidimensional sample excited after a RF pulse, if we apply a gradient $G_{y}$ over a short time interval $t_{y}$ (phase gradient), we obtain different 
phases for each position in the sample. That is, after the short interval gradient, the signal ds $(t, y)$ generated locally from spins in an infinitesimal interval $d y$ at point $y$ without considering $T_{2}$ effects is:

$$
\mathrm{ds}(\mathrm{y}, \mathrm{t})=\mathrm{c} \rho(\mathrm{y}) e^{-i \gamma y G_{y} t_{y}} e^{-i \gamma y B_{0} t} d y
$$

where $\rho(y)$ is the spin distribution along $y$, and $\gamma y G_{y} t_{y}$ represent the phase angle introduced due to the phase gradient $\varphi . \varphi$ is linearly related to the position $y$ in the sample.

Therefore for an arbitrary direction $\mathbf{r}$, and using a $G_{\text {phase }}$ gradient in the $\mathbf{r}$ direction, the total signal received after the application of the phase gradient, is given by:

$$
s(t)=c \int_{-\infty}^{\infty} d s(\mathbf{r}, t)=c \int_{-\infty}^{\infty} \rho(r) e^{-i \gamma r \mathbf{r}_{\text {phase }} t_{r}} d \mathbf{r}
$$

where the carrier signal $e^{-i \omega_{0} t}$ has been removed.

As already mentioned, to solve the phase ambiguities, the entire process of excitation and signal acquisition must be repeated many times, using gradient pulses of incrementally different strengths $G_{r}^{m}=m G_{r}$ by varying $m$. As a consequence, at the location $r_{0}$ the sequence of phase shifts added to a magnetization vector can be written as $\varphi^{\mathrm{m}}\left(\mathrm{r}_{0}\right)=-\gamma \mathrm{r}_{0} \mathrm{mG}_{\mathrm{r}} \mathrm{t}_{\mathrm{r}}$, and therefore the sequence of signal received for each gradient $G^{m}$ is:

$$
s^{m}(t)=c\left[\int_{-\infty}^{\infty} \rho(r) e^{-i \gamma r m G_{r} t_{r}} d \mathbf{r}\right] e^{-i \omega_{0} t}
$$

Using a sequence of $s^{m}(t)$ signals for different values of $m$, we can compute the position of each point of the sample in the $\mathbf{r}$ direction, by using the Fourier transform.

Frequency encoding

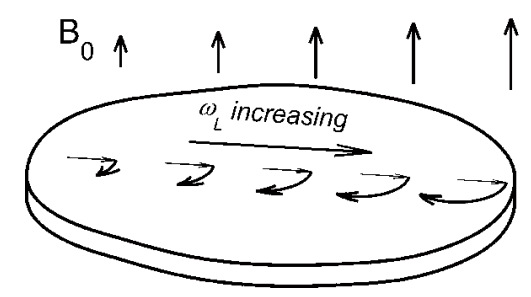

Phase encoding

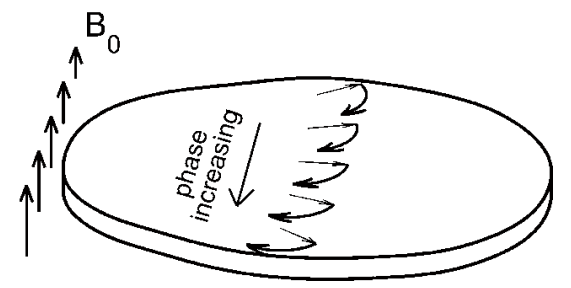

Figure 8: Frequency encoding and phase encoding mechanisms. It is important to notice that the frequency encoding gradient is applied during the acquisition of the signal, while the phase encoding gradients are applied for a short time prior to the signal acquisition. Extracted from [Pra06] with kind permission from Springer Science and Business Media. 


\subsubsection{K-space and magnetic resonance image formation}

According to the encoding techniques explained above we are able to describe the concentration of certain nucleus along a sample volume. If we select a slice of the sample and acquire $M$ frequency encoded FIDs corresponding to different phase encoding gradients, according to equations 25 and 28 we obtain:

$$
s^{m}(t)=c\left[\int_{-\infty}^{\infty} \int_{-\infty}^{\infty} \rho(x, y) e^{-i \gamma\left(x G_{x} t+y m G_{y} t G\right)} d x d y\right] e^{-i \omega_{0} t}
$$

The NMR signals need to be sampled. Therefore the length of $s^{m}$ is equal to the number of samples $\mathrm{N}$ used along reading step. Considering both, the length of $s^{m}(N)$ and the number of phase encoding gradients used $(M)$, we can construct $\mathrm{N} x \mathrm{M}$ matrix including the obtained signals. This matrix is known as k-space.

Considering a sampling interval $\Delta t$ and the demodulated signal, from Eq 29 we obtain the next expression also known as the imaging equation:

$$
s(n, m)=c \int_{-\infty}^{\infty} \int_{-\infty}^{\infty} \rho(x, y) e^{-i \gamma\left(x G_{x} n \Delta t+y m_{y} t_{G}\right)} d x d y
$$

where $0 \leqslant n \leqslant N$ and $-M / 2+1 \leqslant m \leqslant M / 2$.

Considering $k_{x}=\gamma G_{x} n \Delta t$ and $k_{y}=\gamma G_{y} n \Delta t$, the equation 30 can be rewritten as:

$$
s\left(k_{x}, k_{y}\right)=c \int_{-\infty}^{\infty} \int_{-\infty}^{\infty} \rho(x, y) e^{-i\left(x k_{x}+y k_{y}\right)} d x d y
$$

From this expression it is easy to see that we can obtain the spin distribution in the $x y$ plane $\rho(x, y)$ only by applying a two dimensional inverse Fourier transform to the k-space defined by $s\left(k_{x}, k_{y}\right)$. An example of K-space can be seen in Figure 9 

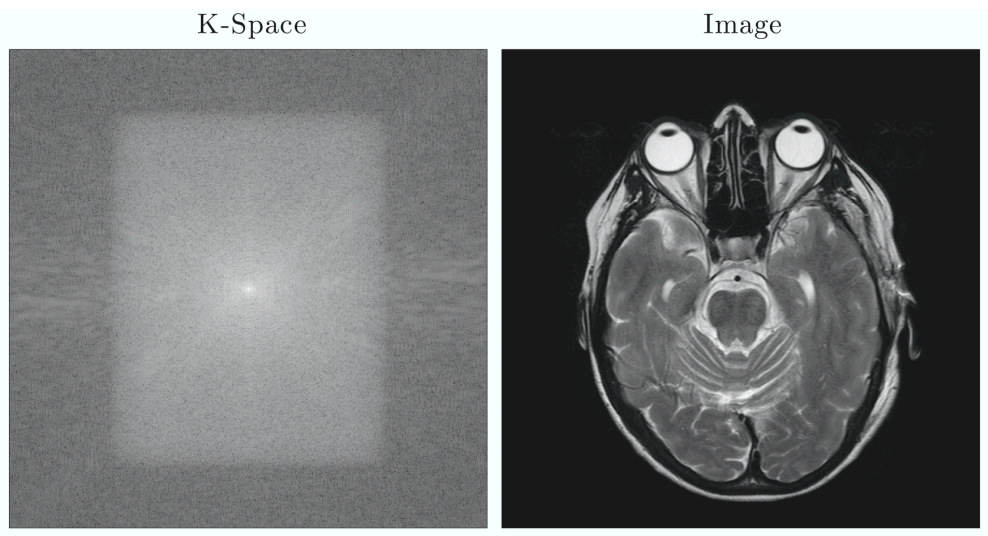

Figure 9: Example of a K-space and its associated image. The image is obtained after the $2 \mathrm{D}$ inverse Fourier transform of the k-space obtained by the scanner. Note that to enhance visualization of K-space, a logarithmic scale has been applied on their intensity values.

Although in this explanation we have considered a slice selection, a phase encoding and a frequency encoding techniques to obtain the signals to obtain k-space data (commonly used for 2D MRI images), other combinations of encoding techniques could used to obtain the k-space information.

\subsubsection{Spectroscopy}

\section{The chemical shift}

Each nuclei of a molecule is influenced by a magnetic field composed by the sum of the external $\mathbf{B}_{\mathbf{o}}$ magnetic field and a local magnetic field $\mathbf{B}_{\mathbf{e n v}}$ of the molecular environment.

Hence, the resonance frequency $v$ depends on the $\mathbf{B}_{\mathbf{0}}$ and $\gamma$, but also on the local environment, that creates a shield of $\mathbf{B}_{\mathbf{0}}$, and represented as $\sigma$,

$$
v=\frac{\gamma \mathbf{B}_{\mathbf{0}}(1-\sigma)}{2 \pi}
$$

This results in different resonance frequencies (or Chemical Shift (CS)) of the same nuclei depending on the molecular environment.

As a consequence of these small differences in the resonance frequencies of each nuclei depending on their molecular environment, it is possible to analyze the composition of a sample by the study of the frequencies reemitted after a RF radiation stimulation. The analysis of this frequencies compound is done by the Fourier Analysis of the reemitted RF radiation.

The frequencies where nuclei resonate are expressed in ppm. This representation is due to the small distance between different resonance frequencies in 
contrast to the high frequencies on which they resonate. Moreover the metabolite peaks positions expressed in ppm are invariant over NMR scanner magnetic field changes, and therefore allow direct comparisons between spectra obtained using different NMR scanners.

The frequency shift $\delta$ in ppm is calculated as

$$
\delta=\frac{v_{\text {nuclei }}-v_{\text {ref }}}{v_{\text {ref }}} \times 10^{6},
$$

where $v_{\text {nuclei }}$ is the Larmor's frequency of resonance of an specific nuclei and $v_{\text {ref }}$ is the Larmor's frequency of resonance of a reference compound, which is usually Tetramethylsilane.

\section{Single voxel spectroscopy}

The Single-Voxel Spectroscopy (SVS) is a NMR technique to obtain information about the chemical composition of a sample by the use of the chemical shift phenomena.

In SVS, a selected region of the analyzed sample is selected and exited by means of RF pulses. After the volume excitation, the FID signal is obtained and analyzed in the frequency domain by applying the Fourier transform to the FID. It is important to notice that although, both MRI and MRS use the frequency information contained in the FID, in MRI the frequency components are generated by the application of a frequency encoding gradient and therefore they are related to the sample spatial profile, and in MRS the frequency components are generated due to the intrinsic frequency shift phenomena and are related to the sample chemical composition.

Because the magnitudes of chemical shift are so small, the acquisition of SVS needs to be done in the absence of frequency encoding gradients. MRS requires an extremely homogenous magnetic field to resolve the spectral peaks.

By mathematical properties of Fourier transform, the widths of the spectral peaks are inversely proportional to $T_{2}^{*}$. Therefore molecules with short $T_{2}^{*}$ values such as lipids, produce broaden spectral peaks.

In SVS, only information from a small volume of the sample is required and therefore no spatial information is needed. This fact simplifies the spatial localization sequences needed to obtain the SVS data. In SVS, the localization is done by a combination of slice-selective excitations. To select a voxel instead of a slice, three slice-selective excitations with different orientations are needed. The voxel selected correspond to the intersection of the three different excited slices.

In the single-shot measurements, the different slice selections applied are made with a single measurement. These single-shots are mostly used in SVS ${ }^{1} \mathrm{H}$ MRS, and require a certain time interval (TE) between the first excitation pulse and the data acquisition. Two types of sequences use single shot 


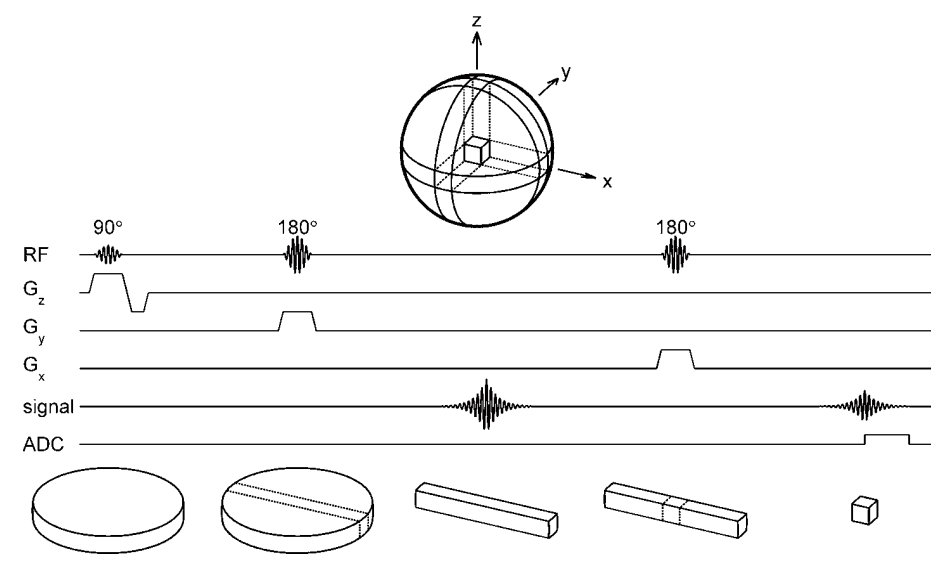

Figure 10: PRESS sequence for the selection of the region of interest in MRS and consisting on a $90^{\circ}$ excitation and two $180^{\circ}$ refocusing pulses. Extracted from [Pra06] with kind permission from Springer Science and Business Media.

measurements: the Point Resolved Spectroscopy (PRESS) sequence consisting on a $90^{\circ}$ excitation and two $180^{\circ}$ refocusing pulses (Figure 10 , and the STimulated Echo Acquisition Mode (STEAM) sequence in which three $90^{\circ}$ pulses are used.

An example of a MRS exam is presented in Figure 11.

\section{Magnetic resonance spectroscopic imaging}

The information provided by MRS about metabolite composition in a selected volume of interest is very useful for the diagnosis of localized pathologies such as the most types of brain tumours. However, in many cases, the knowledge about metabolites concentrations over a large area is mandatory (e.g. neurodegenerative diseases, lesions with diffuse character or multifocal lesions). MRSI, also known as Muti-voxel Spectroscopy (MVS) or Chemical Shift Imaging (CSI), is a NMR technique that allows the acquisition of spectral information from several voxels in different locations defined in a grid.

As was mentioned before, the classical frequency encoding method used in common MRI cannot be applied to obtain chemical shift information from a sample. Therefore, in MRSI only phase encoding methods can be used to obtain the chemical shift information from the sample.

In the simplest case of 1D spatially resolved MRSI using $\mathrm{N}$ voxels, following an excitation pulse, a phase-encoding gradient in the $x$ axis is applied for a time $\tau$. Using the Equation 28 and defining $k_{m}=(\gamma / 2 \pi) \mathrm{G} \tau \mathrm{m}$ (where $\mathrm{m}=-\mathrm{N} / 2 \ldots \mathrm{N} / 2-1$, we can obtain the expression for the MRSI K-space:

$$
S\left(t, k_{m}\right)=\int_{F O V} s(t, x) e^{-i 2 \pi k_{m} x} d x
$$



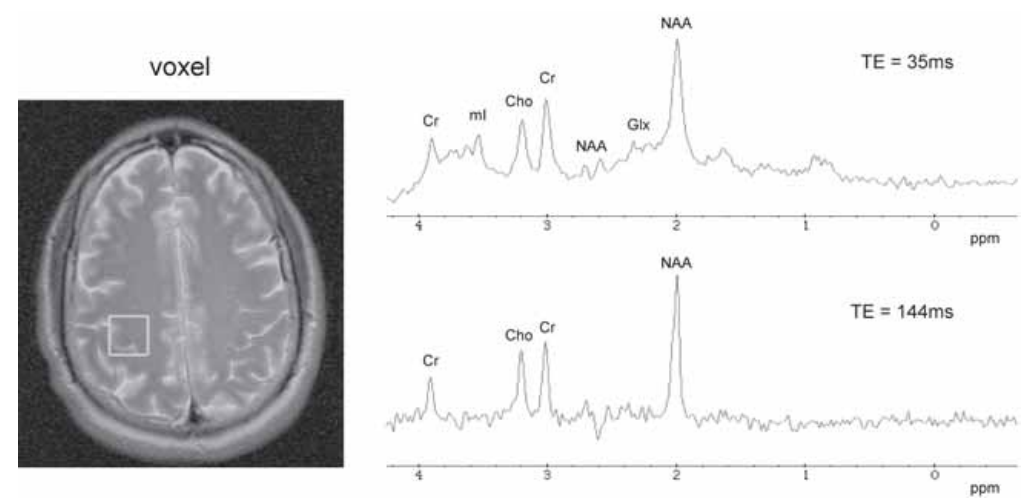

Figure 11: Example of a brain MRS exam. The upper spectrum correspond to a STE acquisition $(T E=35 \mathrm{~ms})$ and the lower spectrum to a LTE acquisition $(\mathrm{TE}=144 \mathrm{~ms})$. Extracted from [Pra06] with kind permission from Springer Science and Business Media.

Multi-voxel spectra

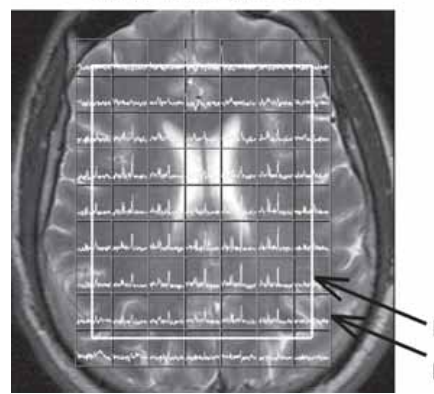

Metabolite maps

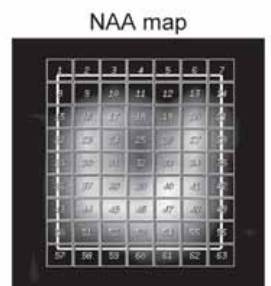

PRESS box

phase-encoding grid

Figure 12: Example of a brain MRSI exam. On the left, the phase-encoding grid and the PRESS box is shown. On the right, the metabolite maps obtained after quantifying the MRSI spectra. Extracted from [Pra06] with kind permission from Springer Science and Business Media. 
The temporal decaying signals associated to the position of the $\mathrm{N}$ voxels can be obtained from the MRSI K-space by the inverse Fourier transform in the $k$ dimension (spatial reconstruction). The chemical shift information can be obtained easily by Fourier transform of each of these temporal signals.

The basic 1-D sequence explained above, can be easily extended to upper dimensions by the application of additional orthogonal phase encoding gradients. The number of voxels or partitions in each spectroscopic grid dimension depends on the number of phase-encoding steps performed in this related direction.

An example of a MRSI study from a brain is presented in Figure 12.

\section{Metabolite concentrations}

Metabolites are the substances produced during the metabolism. Therefore, the study of their concentration in in-vivo analysis is a valuable physiological and pathological tool. This information can be obtained from the MRS techniques. The area of a spectrum peak related to a metabolite is proportional to the number of nuclei from this metabolite in the sample. However, the exact calculation of concentrations is not so simple due to base line distortion effects, superimposed peaks, and noise, among others effects.

The main resonances of the metabolites used in MRS are shown in Table 1, and their mean shape in MRS for different tumour tissues can be seen in Figure 13. 

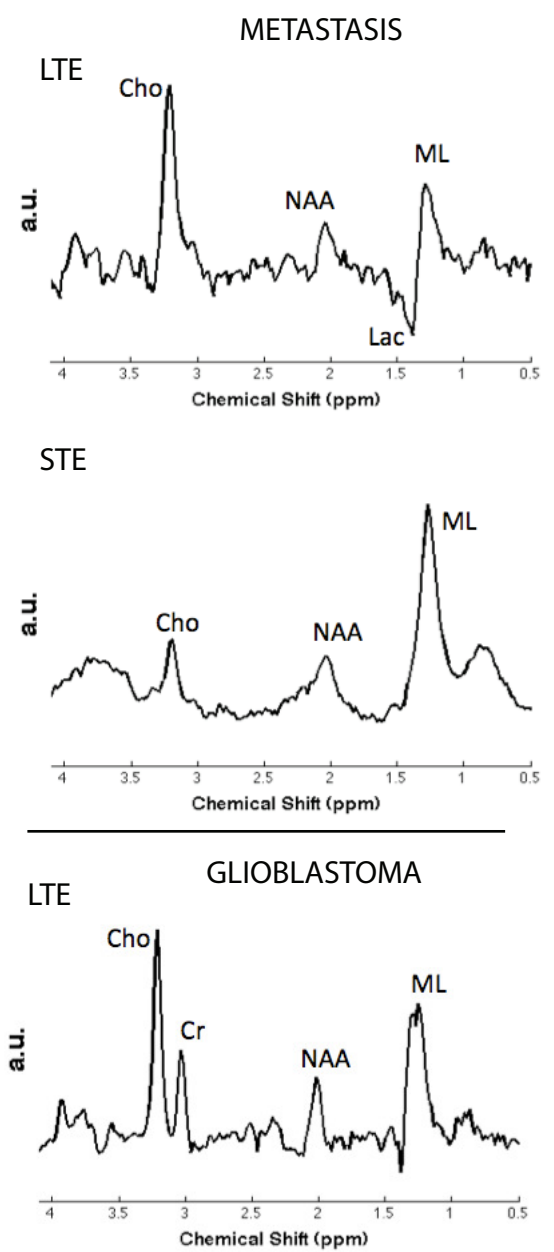

STE

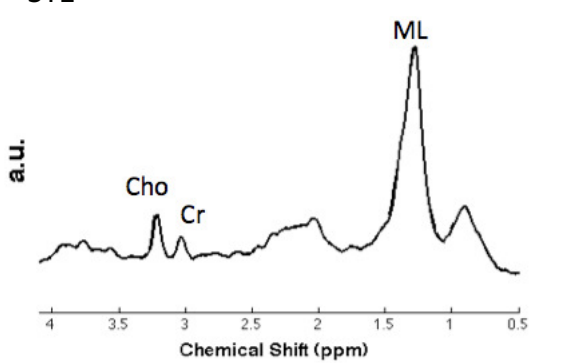

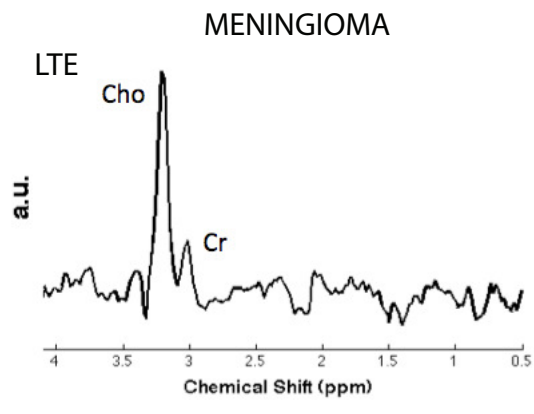

STE
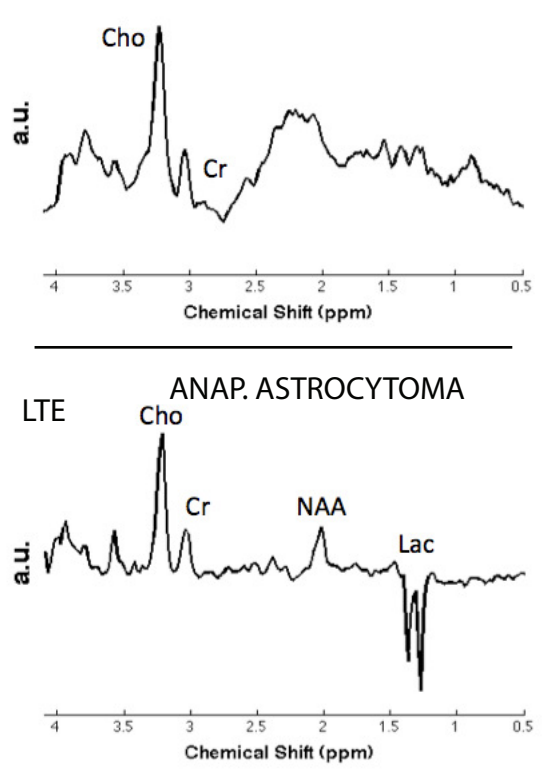

STE

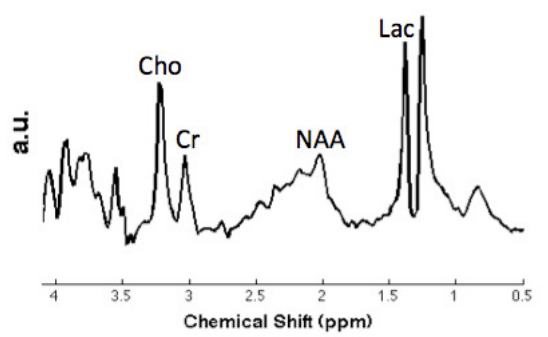

Figure 13: MRS of MET, MEN, GBM and anaplastic astrocytoma brain tumour tissues obtained with LTE and STE. On top left: Proton spectra of a metastasis (43-year-old, male; case et3181 [eTU]). Cho and ML peaks are detected in addition to Lac. On top right: Proton spectra of a low grade meningioma (64-year-old, male; case et3028 [eTU]), showing dominant Cho. Cr and NAA are reduced. On bottom left: Proton spectra of a glioblastoma (73year-old, male; case et2357 [eTU]). On bottom right: Proton spectra of an anaplastic astrocytoma (42-year-old, female; case et3136 [eTU]), showing intensive Cho and Lac. MRS figures kindly provided by Javier Vicente. 
Table 1: Typical ppm of metabolites and other molecules observed in MRS. In this table, the second resonance frequency at which a metabolite can resonate are indicated with (2) symbol

\begin{tabular}{lc}
\hline Metabolite/Molecule & Resonance (ppm) \\
\hline Lipid resonance at $0.92 \mathrm{ppm}$ (L2) & 0.92 \\
Lipid resonance at $1.29 \mathrm{ppm}$ (L1) & 1.29 \\
Lactate (Lac) & 1.31 \\
Alanine (Ala) & 1.47 \\
N-Acetyl groups (NAc) & 2.01 \\
Creatine (Cr) & 3.02 \\
Cr(2) & 3.92 \\
Choline (Cho) & 3.21 \\
Glycine (Gly) & 3.55 \\
Glutamate + Glutamine (Glx) & 2.04 \\
Glx(2) & 2.46 \\
Myo-Inositol (mI)/Taurine (Tau) & 3.26 \\
mI(2) & 3.53 \\
Tau(2) & 3.42 \\
Ala(2) & 3.78 \\
\hline
\end{tabular}

\subsection{ELECTROCARDIOGRAPHY}

The ECG is a noninvasive technique that obtains information about the electric activity of the heart over a period of time. The heart produces a characteristic electric pattern based on the depolarization of the heart muscle during each heartbeat, originated by the synchronized potentials generated at the cellular level. The electrical impulse is generated in the sinus node and transmitted throughout the heart conduction system 15, from atrial cells to ventricular cells. This electric pattern can be detected thought the skin using electrodes as a tiny electrical potential difference that can be amplified and processed by the electrocardiograph.

The potentials generated at the cellular level are originated by a mechanism of cell activation based on the movement of ions $\left(\mathrm{Na}^{+}, \mathrm{Ca}^{2+}, \mathrm{Cl}^{-}, \mathrm{K}^{+}\right)$through the transmembrane ion channels in the cardiac cells, known as cardiac action potential. The cardiac action can be summarized with the following 5 phases (numbered 0-4) represented in Figure 14: (0) Rapid depolarization by opening of the fast $\mathrm{Na}^{+}$channels and later of $\mathrm{Na}^{+} / \mathrm{Ca}^{2+}$; (1) Transient repolarization by rapid exit of $\mathrm{K}^{+}$ions; (2) Plateau by balancing the output of $\mathrm{K}^{+}$and $\mathrm{Ca}^{2+}$; (3) Repolarization, by exit of $\mathrm{K}^{+}$ions while the other channels are closed; and (4) Resting membrane potential. This equilibrium phase is maintained until an external electrical stimulus is applied (typically from an adjacent cell). 


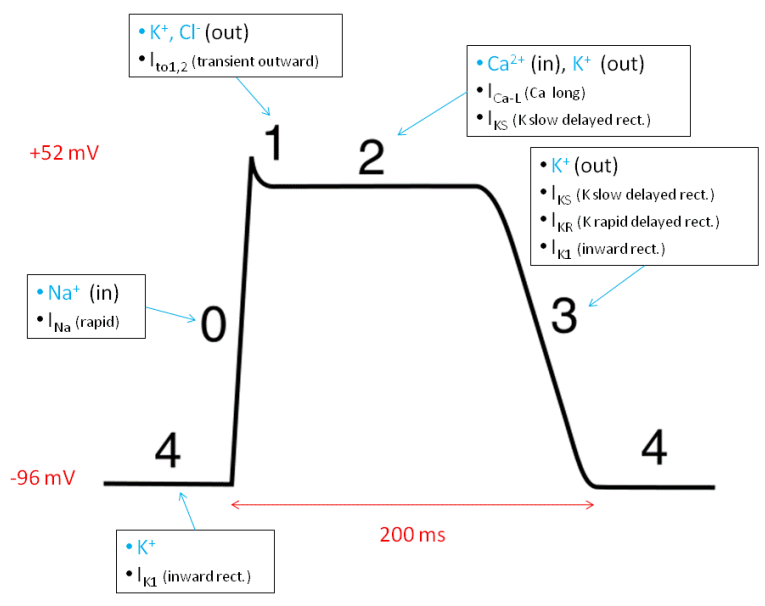

Figure 14: Phases of the cardiac action potential. Extracted from http://en.wikipedia.org/wiki/File: Action_potential_ventr_myocyte.gif.

Consequently, during systole (cell activation and contraction of the muscle) outside the cell is more negative, and during diastole (relaxation of the muscle) outside cell accumulates positive charges. The sum of these synchronized potential variations generates the voltage variations that are detected by ECG though the electrodes.

\subsubsection{The leads}

The electrodes are placed across different areas of the heart and the body in order to obtain information of different directional viewpoints of the heart or leads.

In the standard 12-lead ECG configuration, 12-lead are defined from the 10 electrodes described in Table 2. These leads are divided into three main groups: the limb leads (known as I, II, and III) located in the limbs, the augmented limb leads (known as aVL, aVF, and aVR) derived from the same three electrodes as limb leads but obtaining different different projections of the depolarization vector (or angular viewpoints of the heart), and the precordial leads (known as V1, V2, V3, V4, V5, and V6) placed directly on the chest (see Figures 16,17). 


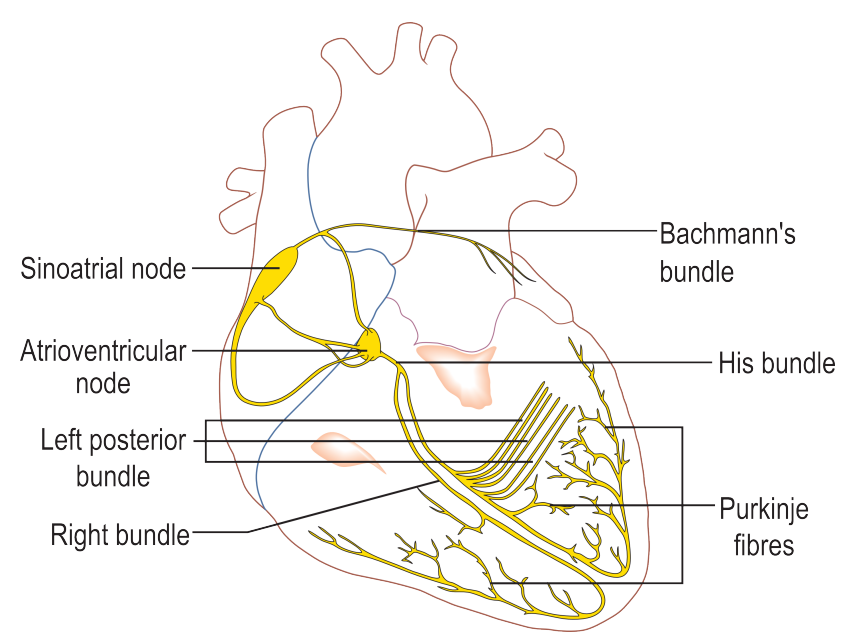

Figure 15: Electrical conduction system of the heart. Extracted from http://commons.wikimedia.org/wiki/File: Conductionsystemoftheheart.png.
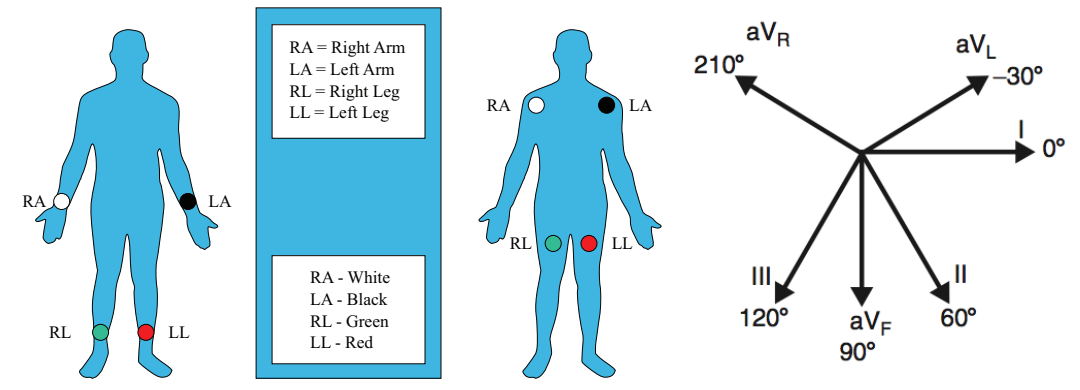

Figure 16: Electrode placements for the limb (I, II, and III) and augmented limb leads (aVL, aVF, and aVR) (left). Different directional views of the heart along the frontal plane are obtained from the limb leads and augmented limb leads (rigth). Extracted from http://commons.wikimedia.org/wiki/File: Limb_leads.svg.
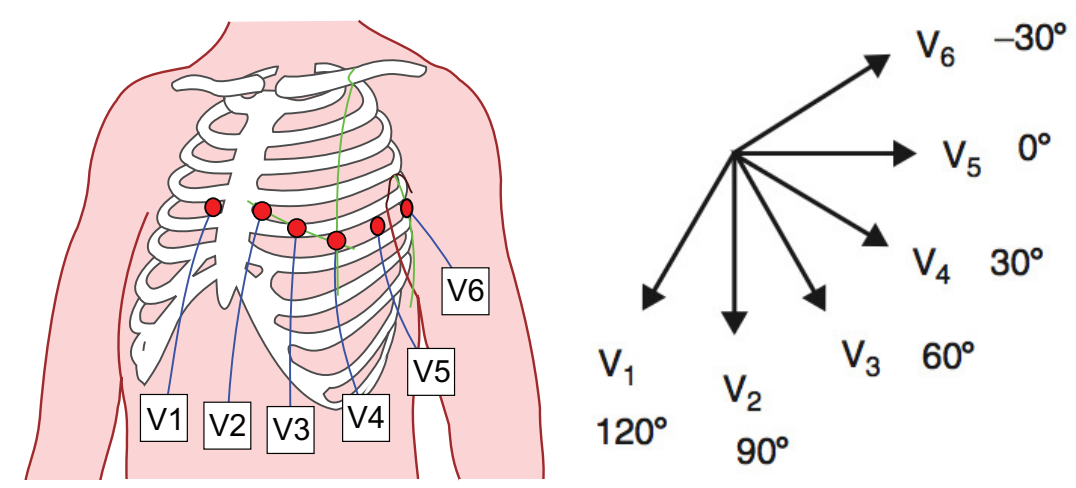

Figure 17: Electrode placements for precordial leads (left). Different directional views of the heart along the frontal plane are obtained from the precordial leads (V1, V2, V3, V4, V5, and V6) (right). Extracted from http://commons.wikimedia.org/wiki/File: Precordial_Leads_2.svg. 


\begin{tabular}{ccccc}
\hline Lead & Electrode + (real) & Electrode - (real) & Signal combination & Angle \\
\hline I & LA & RA & LA-RA & $0^{\circ}$ \\
II & LL & RA & LL-RA & $+60^{\circ}$ \\
II & LL & LA & LL-LA & $+120^{\circ}$ \\
aVL & LA & RA, LL & LA-1/2(RA + LL) & $-30^{\circ}$ \\
aVF & LL & RA, LA & LL-1/2(RA + LA) & $+90^{\circ}$ \\
aVR & RA & LA, LL & RA-1/2(LA + LL) & $-150^{\circ}$ \\
V1 & V1 & LA, RA, LL & V1-1/3(LA + RA + LL) & $+120^{\circ}$ \\
V2 & V2 & LA, RA, LL & V2-1/3(LA + RA + LL) & $+90^{\circ}$ \\
V3 & V3 & LA, RA, LL & V3-1/3(LA + RA + LL) & $+60^{\circ}$ \\
V4 & V4 & LA, RA, LL & V4-1/3(LA + RA + LL) & $+30^{\circ}$ \\
V5 & V5 & LA, RA, LL & V5-1/3(LA + RA + LL) & $0^{\circ}$ \\
V6 & V6 & LA, RA, LL & V6-1/3(LA + RA + LL) & $-30^{\circ}$ \\
\hline
\end{tabular}

Table 2: Combination of electrodes to obtain limb, augmented limb and precordial leads. Note that some leads are known as the same name of the positive electrode that they are obtained from.

\subsubsection{The electrocadiogram signal}

In the ECG the voltage is seen as positive when the direction of the depolarization coincides with the positive electrode of the corresponding lead.

To describe the ECG signal the concepts of waves, segments and intervals are used. (see Fig 18)

WAVES A normal ECG can be described basically by three waves P, QRS and $T$ (see Figure 18). However in some cases $\mathrm{U}$ or Ta waves can be seen in the ECG.

- The P wave corresponds to the electrical activity through the atria.

- The QRS waves (also known as QRS complex), corresponds to the electrical activity through the ventricles,

- The T wave corresponds to the ventricles electrical relaxation. The end of the $T$ wave corresponds with the end of ventricular contraction.

- The Ta corresponds to the atria electrically relaxation. Because it normally coincides with the QRS-complex, it is usually not visible.

- The origin of the $U$ wave is not well known, and is thought to represent re-polarization of endocardial structures or late depolarization of the ventricular myocardium. 
SEGMENTS The segments of the ECG are the sections between the waves. Basically, three segments are used to describe the ECG, these are: the PR segment, the ST segment and the TP segment. The ST segment the time when the ventricle is contracting but no electricity is flowing through it, therefore it usually consists in a straight level line between T wave and QRS complex.

INTERVALS Finally, the intervals represent the time periods between waves. The most characteristic ones are the PR interval, the RT interval, and the R-R interval.

The PR interval values is the time taken by the electrical impulse to travel from sinus node to ventricles through the AV node. The R-R interval it is used to asses the ventricular rate and is measure from a $R$ wave to the next $R$ wave in the ECG. The TR interval is measured from the peak of the $\mathrm{R}$ wave to the start of the $\mathrm{T}$ wave, and is similar to the duration of ventricular systole.

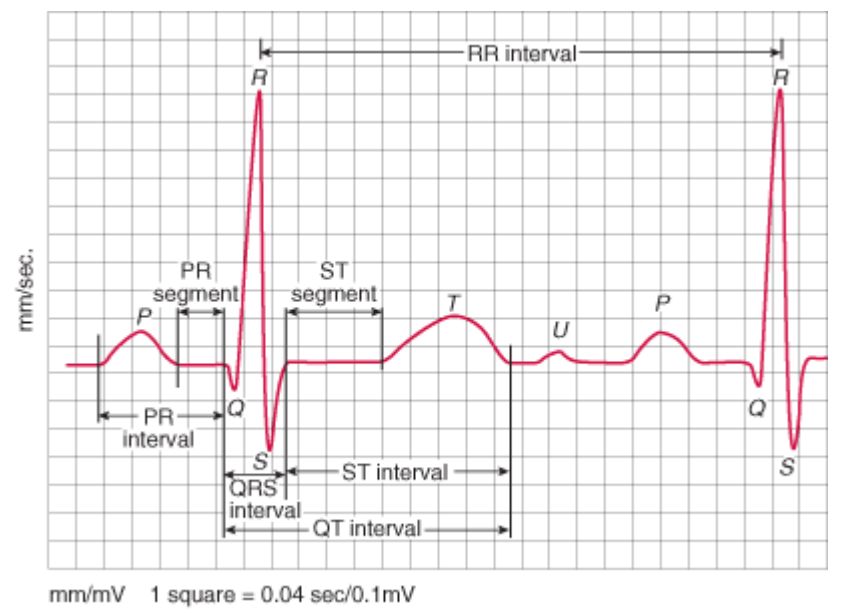

Figure 18: Schematic representation of an standard ECG form a normal heart. On this figure, the localization of waves, intervals, and segments are indicated). Extracted from http://commons.wikimedia.org.

In order to show the relationship between the ECG signal and different parameters measured in the cardiac cycle, a Wiggers diagram is presented in Figure 19. In this figure is easy to see the relationship between ECG, blood pressure, ventricular volume, arterial flow and heart sounds in a cardiac cycle. 


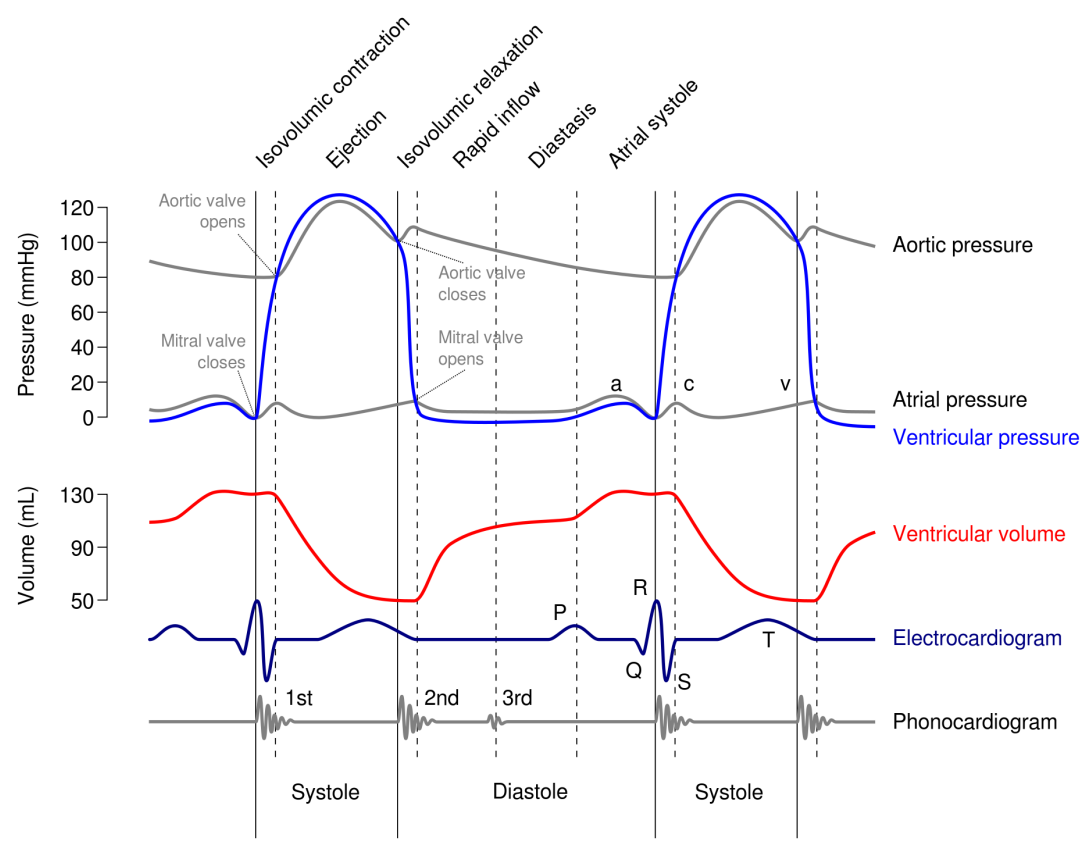

Figure 19: Wiggers diagram. It illustrates the relationship between ECG, blood pressure, ventricular volume, arterial flow and heart sounds in the cardiac cycle. Extracted from http://en.wikipedia.org/wiki/File: Wiggers_Diagram.svg.

\subsection{POSITRON EMISSION TOMOGRAPHY}

PET is a non-invasive in vivo nuclear medical imaging modality that allows the visualization of metabolic activity by generating images of positron emitters. The basic principle of PET imaging is the registration of the radiation emitted from a radionuclides (radiopharmaceutical) distributed along the patient body by external detectors positioned in different orientations. The radionuclides are, in most of cases, introduced in the patient by intravenous injection, and are used to tag specific molecules of interest (molecular tracers). Because the radiopharmaceutical are specific to biochemical pathways or molecular targets, high sensitivity could be achieved. Radionuclides suitable for PET are the short-lived positron-emitting nuclides ${ }^{11} \mathrm{C},{ }^{13} \mathrm{~N},{ }^{1} 5 \mathrm{O},{ }^{18} \mathrm{~F},{ }^{62} \mathrm{Cu},{ }^{68} \mathrm{Ga}$, and ${ }^{82} \mathrm{Rb}$.

After the injection of the radiopharmaceutical, the patient is placed in the Field of View (FOV) of a PET scanner detectors capable to detect incident $\gamma$ rays. Due to the unstable structure of the radionuclides, these proton-rich isotopes may decay via positron emission, in which a proton in the nucleus decays to a neutron, a positron, and a neutrino. The interaction of the neutrino with patient's tissue is totally negligible, however the positron will be rapidly annihilated in a very short distance when reaches thermal energies by the interaction with the surrounding electrons. 
This positron-electron annihilation transforms their mass into energy and produces the back-to-back emission of two $511 \mathrm{KeV}$ photons ( $\gamma$ rays) to conserve energy-momentum (see Figure 20). The energy of these photons allows them to escape from the patient body and to reach the PET detectors without significant loss of energy.

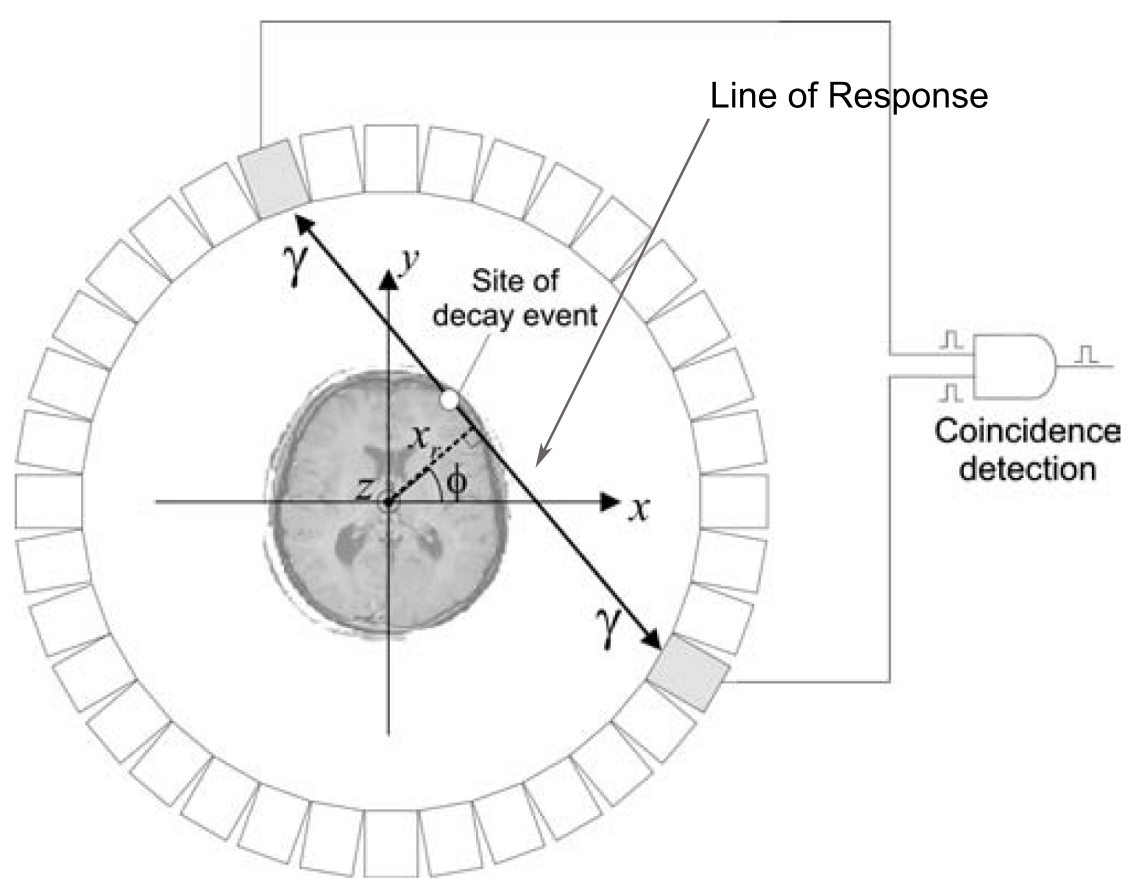

Figure 20: When a decay event is produced, a positron is generated and briefly annihilated with a surrounding electron, producing a back-to-back emission of a pair of $\gamma$ rays. These $\gamma$ rays are detected by the PET scan detectors, and if they are coincident inside a short temporal window, they are labeled as coincidence. The line defined between both detectors is defined as the line of response of the coincidence. Reprinted from [WA04] with permission from Elsevier.

\subsubsection{Coincidence detection}

Each time a $\gamma$ photon interacts with a detector, an electric pulse is registered. When two pulses occur within a short time period $\tau$, are said to be coincident and a line of response is assigned to each coincidence event joining the two detectors (see Figure 20).

In PET scanners, three kinds of coincidences could occur (Figure 21): (A) scattered events in which one or both $\gamma$ photons scatter before arriving to the detector; (B) random coincidences consisting of two $\gamma$ photons generated in different nuclei decays that are detected close enough in time to be coincidence; and (C) true coincidences, consisting of two $\gamma$ photons generated in the same nucleus decay and have not been scattered. 


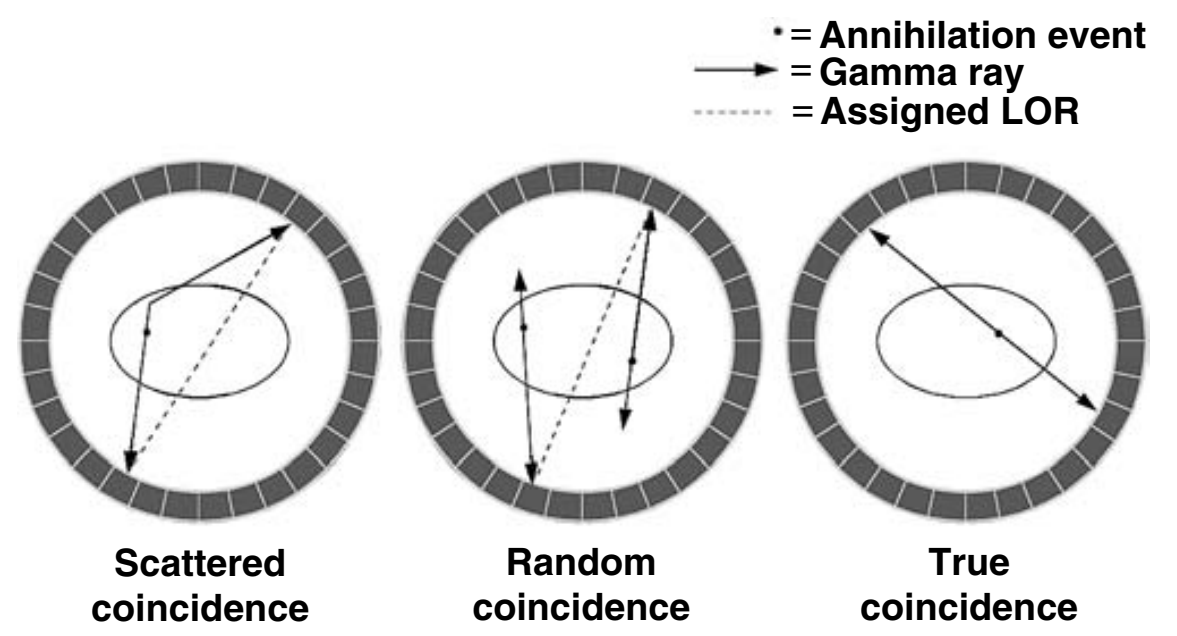

Figure 21: Illustration of the three kinds of coincidences: scattered coincidences, random coincidences, and true coincidence. Reprinted from [WA04] with permission from Elsevier.

The sorting coincidence methods are designed to maximize the percentage of true coincidences in the reconstruction of PET image while minimizing the contribution of random and scattered coincidences. The correctness of these methods affects directly to the improvement of the spatial resolution of the distribution of decay events, and the signal-to-noise ratio of the reconstructed image.

\subsubsection{Image reconstruction}

Once the coincidences are detected and the sorting methods have been applied, the images need to be computed. The reconstruction is needed because the information obtained from the coincidences have been lines of response, and not the activities at certain points. The aim of the image reconstruction algorithms is to recover the activity distribution in the scanner FOV from the projection data.

To represent the raw projection data for a ring of PET detectors a sinogram matrix is used. In the sinogram the number of coincidences obtained for a given detector pair are represented according to the angle of projection $\phi$ (vertical axis) and the displacement $x_{r}$ of the lines of response from the center of the FOV (horizontal axis) of these given detectors (see Figure 20 for the definition of $x_{r}$ and $\phi$ ). That is, each element of the sinogram matrix represents the coincidences of a given detector pair (see Figure 22). 
(a)
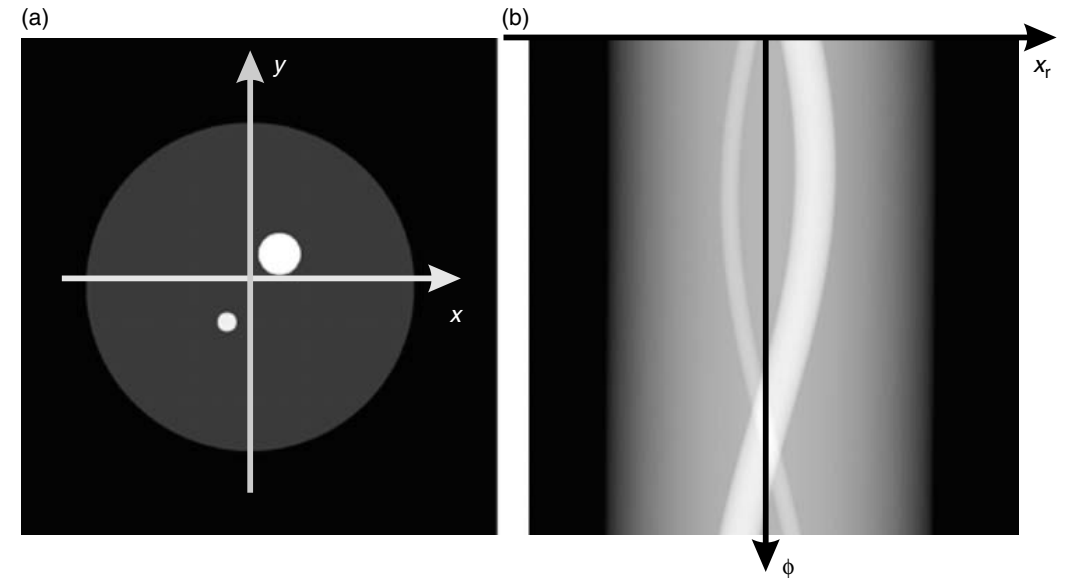

Figure 22: Image of object slice and their associated sinogram matrix represented as a grayscale image. In the sinogram the number of coincidences obtained for a given detector pair are represented according to the angle of projection $\phi$ (vertical axis) and the displacement $x_{r}$ of the lines of response from the center of the FOV. Reprinted from [WA04] with permission from Elsevier.

(a)

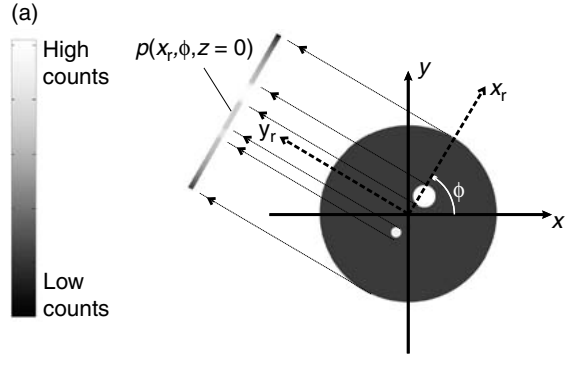

(b)
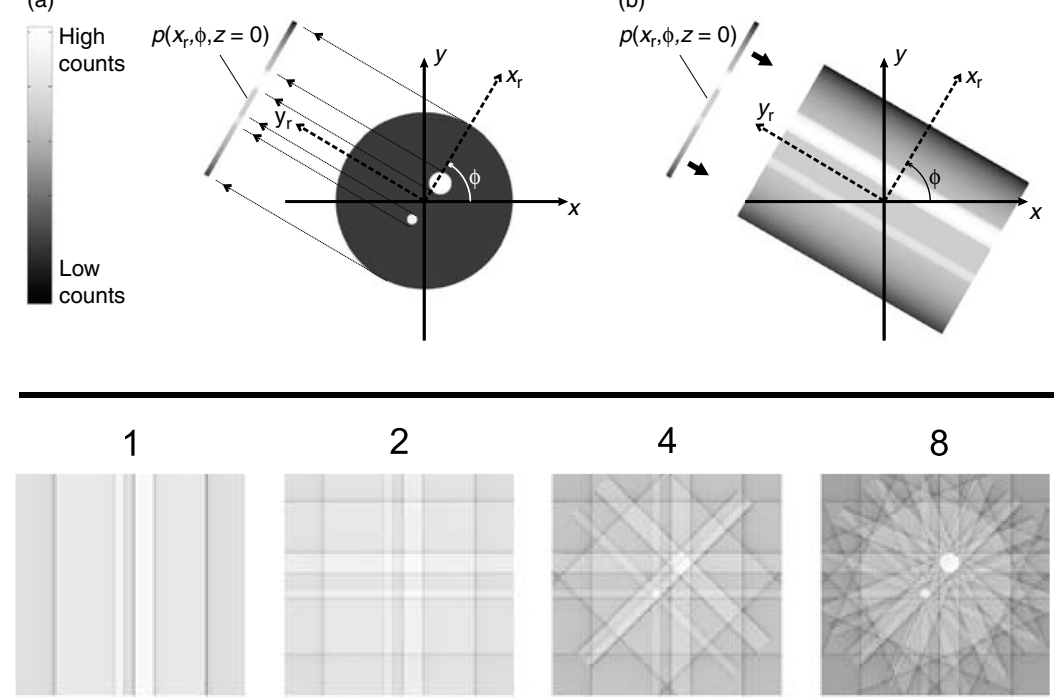

16

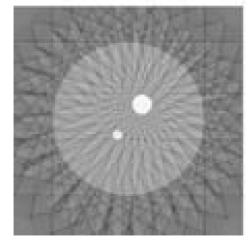

2

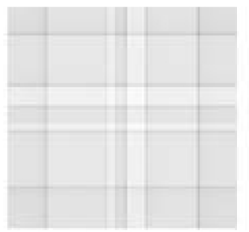

32

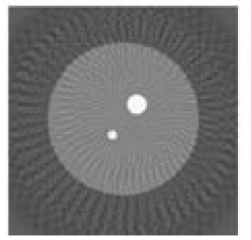

4

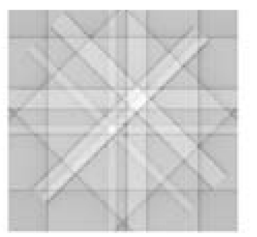

64

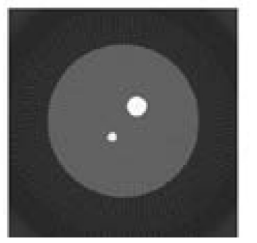

8

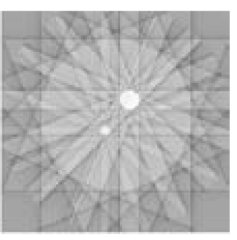

128

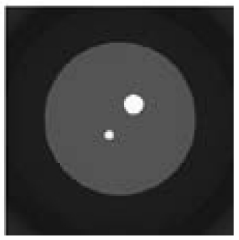

Figure 23: Top: (a) Idealized forward projection of image slice for a particular angle and (b) backprojection for the same angle. Bottom: Reconstruction of the image by filtered backprojection using different number of projections. Reprinted from [WA04] with permission from Elsevier. 
The final PET images need to be reconstructed from the sinogram. In order to illustrate this procedure we will consider for simplicity the basic problem of computing a 3D picture of the patient by computing 2D slices one-byone. The simplest and most widespread reconstruction method to do so is the filtered backprojection method [BDC76]. The backprojection method consist of smearing each projection (horizontal lines of the sinogram) back into the object region along the direction $\phi$ in which it was measured (Figure 23). The sum of all the smearing operations results in a representation of the original object. Nevertheless, due to the assumptions done by the backprojection method the image obtained is blurred (Figure 22). However, this basic reconstruction algorithm produces a blurred image that can be optimized by the use of a sharpening filter. To improve the result obtained by backprojection, the filtered backprojection method includes the application of a sharpening operation to the projections that cancels the blurring effect.

\subsection{CLASSIFICATION OF BIOMEDICAL SIGNALS}

The PR discipline is based on the study of similarities between objects features, to establish relations between sets of such objects. The supervised classification is a specific case of PR task where the goal is identifying a relationship between observations and a set of known categories so that new, previously unseen observations, of unknown categorization, can be assigned a category or class label.

In this thesis the supervised classification PR task is based on a ML approach. In the ML approach, a training set of data containing observations whose category membership is known, is used to fit an adaptive model. Although, the main task in supervised classification is the application of a learning algorithm to obtain a classification model [SIL01], when complex data (as can be the biomedical signals) is used, the consideration of data pre-procesing and transformation as a part of the PR model is critical to optimize the knowledge inference and the robustness of the classification model obtained in the presence of noise and artifacts.

The basic steps needed to build a supervised classification model for biomedical signals and its evaluation are presented in Figure 24 and include: (1) data cleaning and preprocessing, for outliers detection, handle missing data and unlabeled data, de-noising, baseline correction, artifacts elimination, among others; (2) training/test sample selection; (3) feature extraction/selection, to find the useful features with which the training data are more efficiently represented; (4) pattern recognition, to train the supervised classification algorithms used; and (5) evaluation of the classification model obtained by the use of test corpus.

In this section, a brief introduction to the FE/Feature Selection (FS), supervised classification algorithms, and evaluation methodologies used in this thesis, is presented. 


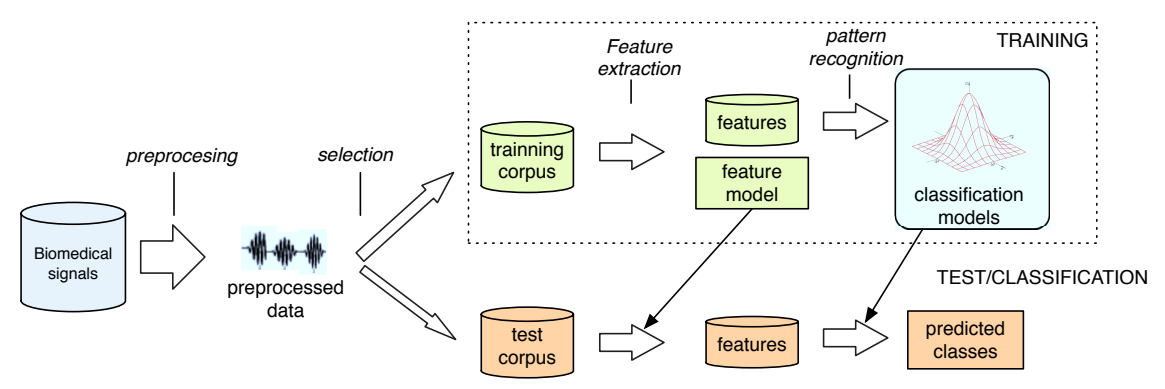

Figure 24: Basic steps to build and evaluate a classification model for biomedical signals.

\subsubsection{Feature extraction and selection methods}

Due to the intrinsic nature of the signals, the high dimensionality problem and non i.i.d. is commonly present on its analysis. To perform statistical analysis based on high dimensionality inputs, large datasets are required. These large datasets are not achievable in practice for most of biomedical problems. Moreover the points that represent the signal shape (variables) contain a high redundant information due to the high correlation between neighboring points. FE and FS methods help us to describe the information contained in a signal by the use of a reduced set of variables. While FE methods reduce signal dimensionality by (linear or non-linear) projection of D-dimensional vector containing signal points values onto d-dimensional vector where $d<D$, FS reduces dimensionality by selecting subset of original variables. In this sense, FE and FS methods could be seen as a kind of dimensionality reduction techniques. ${ }^{1}$

The use of FE and FS reduces the classification model complexity increasing, by this way, the generalization capabilities of the model generated, and it also reduces the number of data needed to train the classifiers. FE and FS could also contribute to the interpretability of the results obtained. Finally they allow faster classifier training and testing.

There are different types of FE and FS depending on whether they are supervised methods or not. While supervised FE and FS methods (e.g. Fisher Criterion (FC), or Recursive elimination of Features (ReliefF)) incorporate knowledge about classes, the un-supervised methods (e.g. Principal Components Analysis (PCA) or Independent Component Analysis (ICA)) do not take this knowledge into account. Moreover, the FE methods can be subdivided into methods based on a multivariate approach and methods based on a functional approach. In this subsection, the most used FE techniques in this work are introduced:

1 Because, FS can be seen as a particular case of FE, for simplicity, in this thesis the term FE will be used for both FE and FS. 
STEPWISE ALGORITHM Stepwise (SW) $\left[\mathrm{RRL}^{+} 06\right]$ is an automatic procedure for statistical variables selection (model selection). After initializing the algorithm with an initial model, SW uses a greedy hill climbing approach, to generate in each step new models by including and excluding single variables. If the maximum of the performance measures in the step improves the performance of the current model plus the tolerance $\epsilon$, then, the new model will be converted in the current model by the inclusion or exclusion of the associated variable, otherwise, the procedure will stop. The procedure will also stop if the maximum number of variables is reached.

RELIEFF Relieff [KSS97] is based on the selection of features that best distinguish between instances that are close to each other. The score assigned to each variable is calculated by the sum of the distance between randomly selected instances and their k-nearest neighbors, of a different class minus the distance to the $k$ neighbors of the same class, and normalized by the range of the variable. A variable will have a high score if large distances have been obtained between samples of different classes and short distances within samples of the same class.

FISHER SCORE The FC method [Fis36] is applied in the context of a classification problem and ranks the discriminative power for each variable by assigning a score value. The score depends on the ability of a variable to separate different classes while keeping a small within-class variance. The FC criterion for feature or spectra point is as follows:

$$
F C=\frac{\sum_{i=1}^{c} n_{i}\left(\mu_{i}-\mu\right)^{2}}{\sum_{i=1}^{c} n_{i} \sigma_{i}^{2}},
$$

where FC denotes the Fisher Score value, $\mathrm{c}$ stands for the number of classes in the dataset, $\mu$ for the global mean value, $\mu_{i}$ denotes the mean value in class $i, n_{i}$ denotes the number of cases in class $i$, and $\sigma_{i}^{2}$ denotes the variance in class $i$.

PRINCIPAL COMPONENT ANALYSIS PCA [Bur04, Fuk90, Jol02] uses an orthogonal transformation, to convert correlated variables into a new linearly uncorrelated ones, assuming jointly normally distributed data. The PCA transformation is defined in such a way, that the first principal component represent the largest possible variance. The higher principal components are defined in the same way, but constrained to be orthogonal with higher principal components already defined.

WAVELET PRINCIPAL COMPONENT ANALYSIS Wavelets are an efficient way to represent the signal information at multiple spatial scales. The wavelet transform [Dau92] consist on carrying out translations and scale transformations of a prototypic wavelet function $\psi$ in order to adjust 
the shape of a signal and to successively obtain the linear expansion of it. Mathematically this can be expressed as:

$$
f(t)=\iint \gamma(s, \tau) \psi_{s, \tau}(t) d \tau d s
$$

where $f(t)$ is the signal and the variables $s$ and $\tau$ are refereed to the translation and scale dimension. The wavelet $\psi_{s, \tau}(t)$ scaled and translated is generated from only one wavelet $\psi(t)$ so called mother wavelet, using the equation 36 .

$$
\psi_{s, \tau}(t)=\frac{1}{\sqrt{s}} \psi\left(\frac{t-\tau}{s}\right)
$$

The wavelet is defined as a finite length or fast decay wave that possess some basic properties, as the admissibility and the regularity condition. These properties imply that the Fourier transform of $\psi(t)$ tends to zero for low frequencies and therefore their behavior is similar to a band pass filter.

Using PCA on the wavelet coefficients of the signal and selecting the principal components with largest variance, allows the dimensionality reduction of signal based on a functional approach.

INDEPENDENT COMPONENT ANALYSIS ICA [HKO01] is a widely used technique for performing blind source separation. ICA is able to generate a basis of signals (sources), from a signal dataset in such a way that the regularity of each basis signal is maximized, and the statistical independence between the signals is maximized.

Given $n$ signals $x_{1}(t), \ldots, x_{n}(t)$, each one composed as a linear combination of $n$ independent sources, $x_{i}(t)=\sum_{j=1}^{n} a_{i j} s_{j}(t), \forall i=1, \ldots, n$, ICA attempts to un-mix the sources $s_{j}(t)$. Let $\mathbf{X}=\left\{x_{i}\left(t_{k}\right)\right\}_{1}^{n}$ the $m \times n$ matrix of the discretized cases $x_{\mathfrak{i}}(t)$, such as, $\mathbf{X}=\mathbf{S A}$, where $\mathbf{S}$ contains the independent sources and $\mathbf{A}$ the linear mixing coefficients. ICA estimates the un-mixing matrix $\mathbf{W}$ that makes $\mathbf{X W}=\mathbf{S}$, that is is able to describe each of the given signals as a combination (defined by $W$ ) of independent sources (defined by $S$ ).

The theoretical foundation of ICA is the Central Limit Theorem, which states that the distribution of the sum (average or linear combination) of independent random variables approaches Gaussian as they increase. So, ICA assumes that the generative model $\mathbf{X}$ tends to be more Gaussian than the sources $\mathbf{S}$. As a consequence, the optimal $\mathbf{W}$ is such that maximizes the nongaussianity of the sources.

Once ICA is obtained for the training dataset, coefficients in the $\mathbf{S}$ basis for a new case $x^{*}(t)=x^{*}\left(t_{1}\right), \ldots, x^{*}\left(t_{m}\right)$ can be easily computed as $\mathrm{W} *=\left(\mathbf{S}^{\mathrm{T}} \mathbf{S}\right)^{-1} \mathbf{S}^{\mathrm{T}} \boldsymbol{\chi}^{*}(\mathrm{t})$.

Most ICA algorithms start with a pre-whitening step based on a PCA of the observations, that is applying a linear transform to the observations so that its 
components are uncorrelated and have unit variance. After pre-whitening, a dimensionality reduction is obtained in the source signal subspace.

\subsubsection{Pattern recognition based classifiers}

The main aim of ML based classifiers or supervised learning methods is to identify the class the new observation belongs to, from the knowledge learned from a training data set containing observations whose classes are known. To do so, the Bayes theorem is used.

The conditional probability tells us that if $A$ and $B$ are two events, the probability of event $A$ when we already know that event $B$ has occurred is,

$$
p(A \mid B)=\frac{p(A \cap B)}{p(B)} \text { if } p(B)>0
$$

So, the probability of $A$ given $B$ can be seen as the event $A$ becomes $A \cap B$ but considering that we know that $B$ was happened, and therefore dividing it by the $\mathrm{p}(\mathrm{B})$.

The Bayes Theorem or Bayes Rule is based on this conditional probability. The Bayes Theorem, when used in statistical PR, says that the probability of class $\omega_{j}$ ( $j$-th class) given the feature/observation vector $\mathbf{x}$ is,

$$
p\left(\omega_{j} \mid \mathbf{x}\right)=\frac{p\left(\mathbf{x} \cap \omega_{j}\right)}{p(\mathbf{x})}=\frac{p\left(\mathbf{x} \mid \omega_{j}\right) p\left(\omega_{j}\right)}{p(x)}
$$

Each term in the Bayes Theorem has a name: $p\left(\omega_{j}\right)$ is known as prior probability (of class $\left.\omega_{j}\right) ; p\left(\omega_{j} \mid \mathbf{x}\right)$ is known as posterior probability (of class $\omega_{j}$ given the observation $\left.\mathbf{x}\right) ; \boldsymbol{p}\left(\mathbf{x} \cap \omega_{j}\right)$ is the likelihood (probability of observation $\mathbf{x}$ given class $\left.\omega_{j}\right)$; and $p(\mathbf{x})$ is known as normalization constant (does not affect the decision).

The Bayes decision rule consists into choosing class $\omega_{i}$ with highest $p\left(\omega_{i} \mid \mathbf{x}\right)$, that is assigning each case $\mathbf{x}$ of the sample to the class $\omega_{i}$ that maximizes the posterior probability $p\left(\omega_{i} \mid \mathbf{x}\right)$

$$
\hat{\mathbf{c}}=\arg \max _{\omega} p(\omega \mid \mathbf{x})=\arg \max _{\omega} p(\mathbf{x} \mid \omega) p(\omega)
$$

The supervised classification problem consists in, given a finite sample of cases, approximating the real probability distribution. To do so, different statistical-based and non-statistical-based classifiers have been used in this thesis to classify biomedical signals. In this section, a brief description of the classification algorithms used in this thesis is presented. These supervised learning algorithms are widely used in Level 4 CDSSs [Bra02]. 

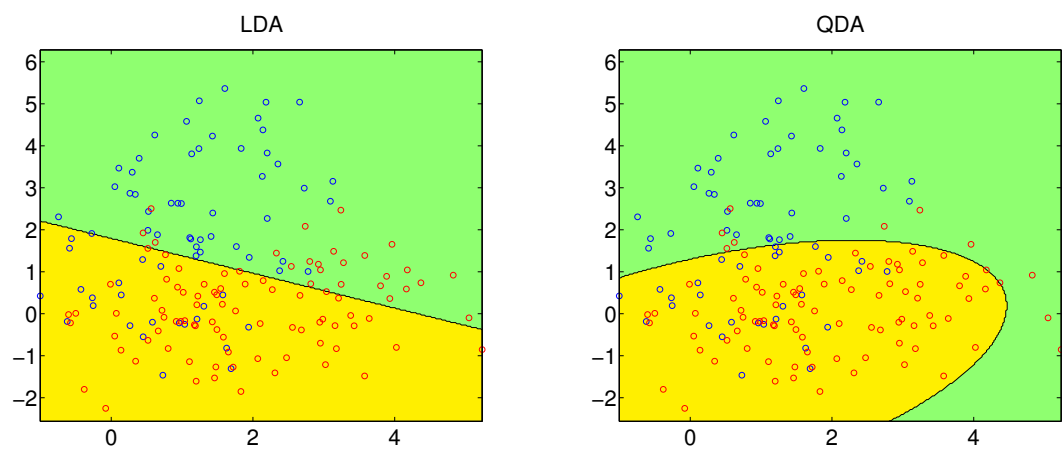

KNN (k=1)
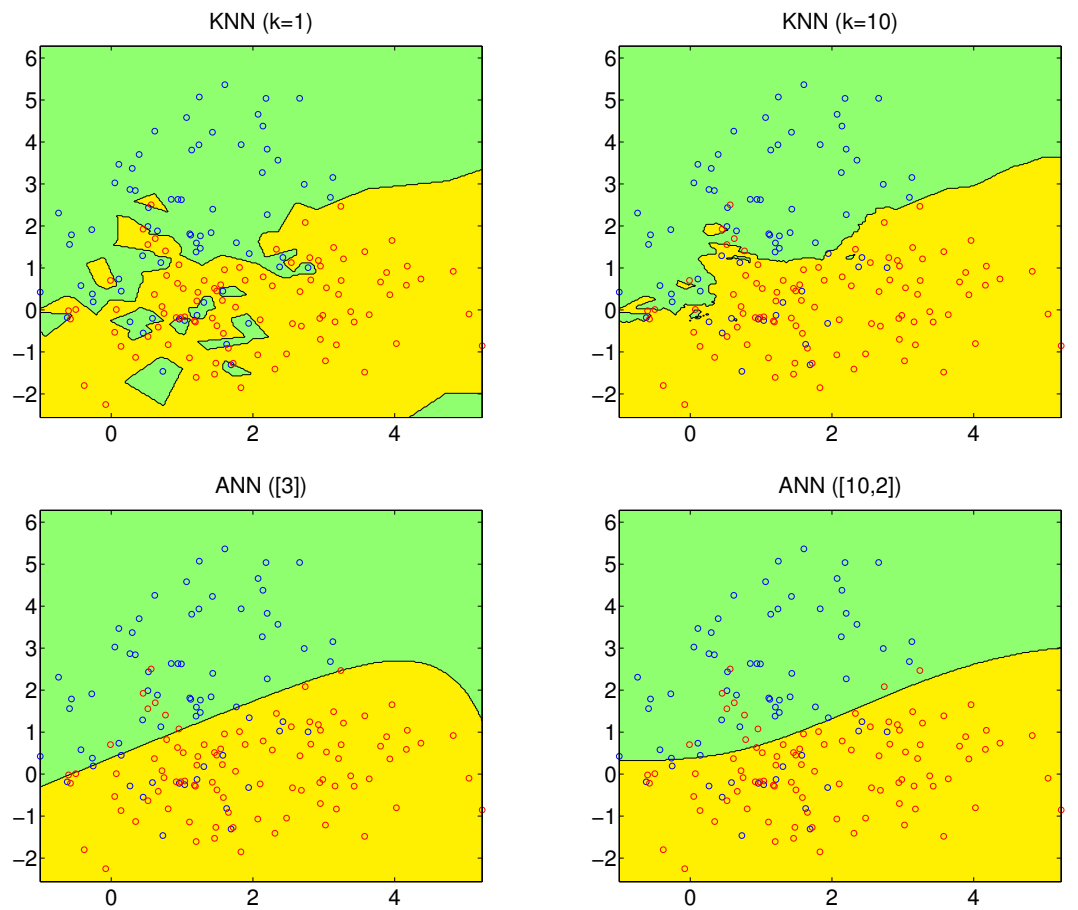

Figure 25: Classification boundaries obtained by the classification methods included in this thesis (LDA,QDA, KNN,ANN) for a two classes training dataset. It is easy to observe the lineal and quadratic frontiers generated by LDA and QDA (top). Moreover, it is important to see how the KNN increases the smoothness of its boundary as the number of neighbors $k$ is smaller (middle). Finally, the boundaries obtained by two basic MLP ANNs consisting on a single hidden layer (with 3 neurons), and two hidden layer (with 10 in the first layer and 2 neurons in the second layer) are presented (bottom). As can be seen, the more complex ANN configuration is able to generate more complex boundaries. Notice that, for illustration purposes, the boundaries obtained are plotted over the training dataset and not over an independent test. Therefore, boundaries as the one generated by KNN $(k=1)$ probably are overfitted to training data. 
FISHER LINEAR DISCRIMINANT ANALYSis Fisher's LDA [DHS00] is a classification technique that finds the linear combination of features that best separates the classes of objects (see Figure 25). It consists of a ratio between the difference of the projected means and a measure of dispersion of each class. This function is optimal when the distance between means is maximal and the inside class dispersion is minimal.

QUADRATIC DISCRIMINANT ANALYSIS BASED ON MAHALANOBIS DISTANCE QDA [DHS00] is closely related to LDA. Although in both analysis there is an assumption of normally distributed data inside each class, in QDA it is not assumed that the variances of each class are equal, and the resulting separating surface between the classes is a quadratic (see Figure 25). In QDA new objects are classified according to the class for which it has the smallest Mahalanobis distance.

K-NEAREST NEIGHBORS ( KNN) The KNN [DHS00] algorithm is an instance-based method for classifying objects based on the $k$ closest training examples in the feature space given a metric (see Figure 25).

ARTIFICIAL NEURAL NETWORKS ANN are data models composed by an interconnected group of simple processors that work in parallel to transform the information from the input to the output [Bis95]. The mostly used type of ANN is the MLP, a feedforward artificial neural network model, consisting on multiple layers of perceptrons with each layer fully connected to the next one. If the MLP ANN has no hidden layers, then it can only learn linear problems. If it has one hidden layer, then it can learn problems with convex decision boundaries (and some concave decision boundaries). Finally with two or more hidden layers the network is able to learn more complex problems (see Figure 25).

In a MLP for solving a classification problem, the input signal propagates through the perceptrons layer-by-layer obtaining an approximation of the probability distribution of each class. MLP is able to distinguish data that is not linearly separable.

\subsubsection{Evaluation strategies}

Once a classification model is trained one may be tempted to use the entire training dataset to evaluate the classifier performance, and to select the one that best perform the classification task. However this approach has two fundamental problems that cannot be ignored. First the performance values obtained will be over-optimistic, and second the model selected will normally not be able to generalize to new data because it has overfitted the training data. 
The evaluation strategies, help us to validate supervised classification models by the selection of different training/test corpus.

HOLDOUT METHOD The holdout method consist into split dataset into two groups, one for train and the other for test, This basic evaluation strategy has two main drawbacks: It does not use all the available data for test and is very dependent on the split done.

RANDOM SUBSAMPLING Random subsampling perform $k$ train-tests experiments. In each of this experiments it select randomly a fixed number of cases for test and exclude them in the training dataset. The final true error estimates is obtained as the average of the k error estimates obtained for each experiment.

K-FOLD CROSS VALIDATION K-fold cross validation creates a K-fold partition of the dataset, and perform $k$ train-test experiments, using $\mathrm{K}-1$ folds for training, and the excluded one for test. As before, the final true error estimates is obtained as the average of the $k$ error estimates obtained for each experiment.

LEAVE-ONE-OUT CROSS VALIDATION Leave-one-out methods is a special case of $k$-fold cross validation, where $k$ is equal to the number of cases. This method is particularly useful in case of very sparse datasets, or very small datasets, where the use of many cases as possible is required for training the model.

The advantage of $K$-fold cross validation and Leave-one-out methods with respect to holdout or Random subsampling is that all the cases in the dataset are used for both training and testing.

\subsubsection{Figures of merit}

A figure of merit is a measure used to characterize the performance of a classification model. There are several measures for determining the performance of a classifier. In this thesis the following evaluation metrics are used:

ACCURACY (ACC) ACC is defined as $\frac{\mathrm{N}^{+}}{\mathrm{N}}$, where $\mathrm{N}^{+}$is the number of samples correctly predicted by the classifier and $\mathrm{N}$ is the total number of samples used for testing.

ERROR RATE (ERR) ERR is defined as $\frac{\mathrm{N}^{-}}{\mathrm{N}}$, where $\mathrm{N}^{-}$is the number of samples incorrectly predicted by the classifier. ERR $=1-$ ACC 
GEOMETRIC MEAN OF RECALLS ( $G) \quad G$ it is defined as the $|C|-$ th root of the product of all the successful predictions for each type of class, where $|C|$ is the total number of classes (Equation 40). This nonlinear metric is especially useful to avoid overestimating the classifier performance when predictions show a high variance over classes. It will be high if and only if the accuracy of each class is high and they are in equilibrium.

$$
G=\sqrt[|c|]{\prod_{c} \frac{N_{c}^{+}}{N_{c}}}
$$


Part II

CONTRIBUTIONS 

DESIGN AND IMPLEMENTATION OF BIOMEDICAL SIGNAL PROCESSING AND VISUALIZATION SOFTWARE

One of the basic task in a biomedical signals classification system based on PR is the automatic signal pre-processing task (see Section 2.5). The signal pre-processing is crucial to remove artifacts, reduce noise, or improve the interpretation of the information contained in the signals. Moreover, the automatic pre-processing module ensures that the transformations applied to new cases will be the same that the one used with the dataset used to train the classification module. This is crucial to ensure the reproducibility of the performances obtained during the design of the classifier as well as to increase robustness in front of major signal artifacts.

With this aim, three contributions to automatic biomedical signal preprocessing have been included in this thesis. Each one of these contributions is described in each section of this chapter. In the first section, the design and implementation of an open source MRSI toolbox MRSIlab for the pre-pocess, analysis and visualization of MRSI data is presented. In the second section we will focus on the design of a semi-automatic preprocessing module for CURIAM-BT CDSS for brain tumours classification by MRS based on EC project, contract no. FP5-1999-IST-10310 (INTERPRET) pre-processing protocol. Finally, in the last section the design and implementation of an software for ECG pre-processing, segmentation, visualization, and labeling is described.

MRSILab has been successfully tested and used in different research projects and studies by Katholieke Universiteit Leuven, St George's, University of London and Polytechnic University of Valencia. CURIAM-BT CDSS and its preprocessing pipeline are registered by the Polytechnic University of Valencia, it has been presented in different congresses [SGGV $V^{+} 09, S G G V^{+} 08$ ], and it was evaluated at the Hospital Universitario Doctor Peset and Hospital de la Ribera [SGGAB ${ }^{+}$12]. Arrhythlab is being registered, and has been tested and successfully used at Sistema Experto en Electrocardiografía project (TSI020302-2010-136). 


\subsection{DESIGN AND IMPLEMENTATION OF MRSILAB: A MRSI PRO- CESSING AND VISUALIZATION MATLAB TOOLBOX.}

\subsubsection{Motivation}

During the last decade the MRSI technique has become widely used in clinical practice, complementing the information of MRI. MRSI combines the properties of MRI and MRS techniques obtaining, not only information about the geometry of the affliction and the different degrees of development inside the affected region, but also information about metabolites concentration in each point of the image.

Despite this, the development of open source software platforms with capabilities for processing and analyzing such data has been hindered due to the lack of a standard raw data file format that facilitates the reading of data from the NMR scanner. These open source software platforms allow quick development of new techniques for analysis and processing of MRSI, and enormously simplify the design of multicenter studies involving MRSI datasets obtained by NMR scanners from different manufacturers. The few currently available software platforms capable of working with most commonly used MRSI file formats $\left[\mathrm{ZPAM}^{+} 05, \mathrm{KMN}^{+} 06, \mathrm{CON} 09\right.$, $\mathrm{VVV}^{+} 96$ ] are not as frequently updated as desired and, therefore, do not allow the reading of the latest formats. In addition, in most cases these pieces of software are proprietary source, making even more difficult its use to serve the needs of technical researcher.

With the objective to contribute to the development of new methods and algorithms for MRSI processing and analysis, an open source MRSI toolbox MRSILab has been developed. It has been implemented in MATLAB to facilitate the testing of novel methods and algorithms and it has been included in a virtual working environment. This toolbox currently offers several algorithms implemented over the last years through the collaboration between research centers in the framework of different projects [The07, GVMJS ${ }^{+}$07].

\subsubsection{MRSILab modules}

MRSILab is composed of different modules that handle tasks concerning to: (1) reading MRSI data in RAW format, (2) MRSI pre-processing, (3) MRS preprocessing, (4) metabolite quantification, (5) MRSI-MRI co-registration, and (6) visualization.

Each part of this section details the processes contained in each of these modules. In Figure 26, a schema of MRSILab is presented. In this figure, each task included in each of the modules, as well as the connection between them, are presented. 


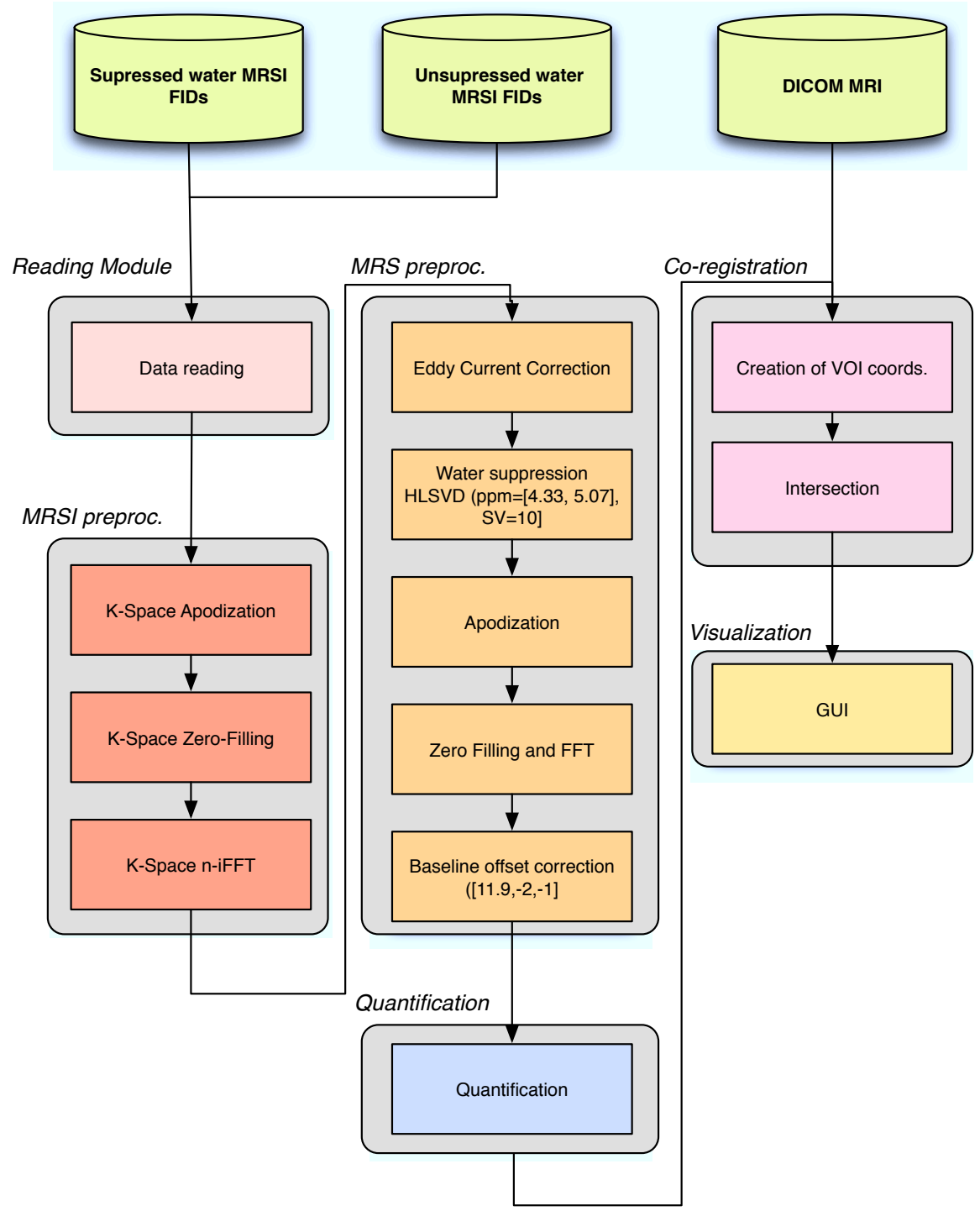

Figure 26: MRSIlab schema 


\subsubsection{Reading module}

As already mentioned, one of the main difficulties in the development of new algorithms and methods for MRSI processing and analysis is the lack of a standard raw data file format hampering access to NMR scanner data. Each manufacturer uses its own file format to codify the raw data, and even in the case of MRSI in DICOM format, the slow adoption of the standard for MRSI does not facilitate the localization of the raw data and its properties inside the binary file. Therefore MRSI data acquired by the scanner is saved in DICOM's private tags that vary from vendor to vendor.

In addition, the coordinate system used in the MRSI metadata, most of times does not coincide with the MRI coordinate system and therefore a coordinate transformation is needed to compare the position of the two image modalities. This coordinate transformation not only depends on the proprietary fileformat but also in its version, making difficult the analysis of MRSI data by third party software.

Current version of MRSILab toolbox, it is able to read Philips SPAR and SDAT 2D-3D formats, and General Electric .7 and .SHF formats (single and multi-coil). In the following paragraphs a detailed description of these file formats and their structure is presented.

\section{Philips SPAR and SDAT 2D-3D formats and versions}

Philips MRSI raw data is encoded, in most of cases, using two files. The first one uses a .SDAT extension and contains the binary MRSI data from the scanner. The second one uses a .SPAR filename extension and contains the metadata related to the acquisition parameters and clinical study information in plain text.

- Reading metadata .SPAR files

The metadata described in .SPAR files are encoded as plain text and the reading procedure is easy. However the interpretation is not as easy because it is not documented. In this work a documentation of the most important fields in the .SPAR files are included, this information has not been described in any public Philips manual and has been obtained by the experience. In table 3 a selection of fields contained in the .SPAR file and needed for the reading, processing and registering of the MRSI data are presented. The name of each of these fields is described in the first column while the second column includes their description.

It is important to notice, that although the fields and their meaning remain constant along the different versions of .SPAR files, the coordinate system used to codify the position of MRSI analysis may change between file versions. In this work we have focused our analysis in the Philips versions mostly used along the projects we were involved 
Table 3: Description of the .SPAR file fields used for MRSI data reading, processing and registration.

\begin{tabular}{|c|c|}
\hline Field in the.$S P A R$ file & Description \\
\hline samples & number of points in the temporal dimension \\
\hline \multirow[t]{2}{*}{ rows } & no. of voxels in the $x$ direction $\times$ \\
\hline & no. of voxels in the y direction \\
\hline synthesizer_frequency & synthesizer frequency \\
\hline sample_frequency & Sampling frequency \\
\hline echo_time & echo time (ms) \\
\hline repetition_time & repetition time $(\mathrm{ms})$ \\
\hline ap_size & anteroposterior ROI size $(\mathrm{mm})$ \\
\hline lr_size & left-right ROI size $(\mathrm{mm})$ \\
\hline cc_size & craneocaudal ROI size $(\mathrm{mm})$ \\
\hline ap_off_center & anteroposterior ROI off_center displacement $(\mathrm{mm})$ \\
\hline lr_off_center & left-right ROI off_center displacement (mm) \\
\hline cc_off_center & craneocaudal ROI off_center displacement (mm) \\
\hline ap_angulation & anteroposterior ROI angulation (degrees) \\
\hline lr_angulation & left-right ROI angulation (degrees) \\
\hline cc_angulation & craneocaudal ROI angulation (degrees) \\
\hline volume_selection_method & volume selection method \\
\hline si_ap_off_center & anteroposterior FOV off_center displacement (mm) \\
\hline si_lr_off_center left-right & FOV off_center displacement $(\mathrm{mm})$ \\
\hline si_cc_off_center & craneocaudal FOV off_center displacement (mm) \\
\hline si_ap_off_angulation & anteroposterior FOV angulation (degrees) \\
\hline si_lr_off_angulation & left-right FOV angulation (degrees) \\
\hline si_cc_off_angulation & craneocaudal FOV angulation (degrees) \\
\hline phase_encoding_fov & width of the FOV in the $y$ direction $(\mathrm{mm})$ \\
\hline slice_thickness & slice thickness $(\mathrm{mm})$ \\
\hline nr_of_phase_encoding_profiles_ky & no. of voxels in the $y$ direction \\
\hline nr_of_slices_for_multislice & number of voxels in the $z$ direction in case of $3 D M R S I$ \\
\hline
\end{tabular}


in. These versions are: 2004-2006 (v2.1.3), 2006 (v2.5.3) or 2007 (v2.6.1-v2.6.3), and 2008. Based on the work of Montelius in [Mon08], we developed some transformations to compare between coordinates of different versions. In this work the transformations were obtained using a MRSI-MRI phantom to reveal the directions of the image coordinate system for the Philips scanner.

In the 2004-2006 (v2.1.3), the equations 41, 42 compensate for the change of signs between the VOI and image coordinate systems.

$$
\begin{aligned}
\text { ap_off_center } & =- \text { ap_off_center; } \\
\text { lr_off_center } & =-l_{-} \text {off_center }
\end{aligned}
$$

In the 2006 (v2.5.3) or 2007 (v2.6.1-v2.6.3), the equations 43,44 change the rotational direction around the anteroposterior and left-rigth axes.

$$
\begin{aligned}
\text { ap_angulation } & =- \text { ap_angulation; } \\
\text { lr_angulation } & =-l r_{-} \text {angulation; }
\end{aligned}
$$

In posterior versions, the equations $45,46,47$ compensate for the change of signs between the VOI and image coordinate systems, and the equation 48 change the rotational direction around the craneocaudal axis.

$$
\begin{aligned}
\text { ap_off_center } & =- \text { ap_off_center; } \\
\text { lr_off_center } & =- \text { lr_off_center; } \\
\text { cc_off_center } & =- \text { cc_off_center; } \\
\text { cc_angulation } & =- \text { cc_angulation; }
\end{aligned}
$$

\section{- Reading MRSI data in .SDAT files}

The encoding format of .SDAT files is not documented. Although in the .SPAR files is documented the use of the instruction set architecture Virtual Address eXtension (VAX), the order of the MRSI data and the data structure is not specified. It has been necessary to use the MRSI multicenter dataset collected along different projects to determine this information by reverse engineering. As a result of this work it has been determined that SDAT files use IEEE-LE format with a 32bits VAXD floating point representation.

Once the binary data has been loaded, a large vector of numbers is obtained and therefore we have to determine their order and structure 
Table 4: Description of the .SHF file fields used for MRSI data reading, and processing.

\begin{tabular}{ll}
\hline Field in the .SHF file & Description \\
\hline opuser5 & no. of voxels in the $x$ direction \\
opuser6 & no. of voxels in the y direction \\
opuser7 & no. of slices \\
num_coils & number of coils used \\
opuser1 & number of points in the temporal dimension \\
center_freq & synthesizer frequency \\
is_chopped & if is_chopped=1, then it is needed to reorder the voxels \\
opuser0 & spectral width (KHz) \\
data_format & file version \\
header_size & header size in the .7 file \\
offset_to_data & offset to data in the .7 file \\
\hline
\end{tabular}

to reconstruct the MRSI k-space. The fist step consists on separate the vector values located in odd positions from the values located in even positions. The first ones represent the real values, while the second ones the imaginary values of the complex MRSI signal. The second step consists on rearrange the vector in a 3D or 4D hyper-matrix structure (depending on whether it represents 3D or 2D MRSI), consisting in 1 temporal dimension and 2 or 3 phase encoding dimensions.

\section{General Electric .7 and .SHF formats single and multi-coil}

The proprietary file formats used by General Electric for MRS and MRSI spectroscopy use .7 and SHF file extensions. In this case, the .SHF file contains the MRSI metadata and the .7 contains the MRSI binary data.

- Reading metadata .SHF files

The .SHF GE file specifies the type of encoding used in the .7 file. Moreover it contains relevant information for the reading and processing of the MRSI data in the .7 binary file. The most relevant information is resumed in Table 4.

- Reading MRSI data in .7 files

The 7 files use the IEEE-BE format for Raw 7.x thru 9.0 CSI version while IEEE-LE for Raw 12.0 CSI or Raw 15.0 CSI Multicoil versions with a 32 bits integer codification. To read the .7 file we have to take into account the offset_to_data specified on.SHF file and described on Table 4, to know in which part of the file we have to begin to read the data. Once the binary MRS data are read we follow the above mentioned procedure defined for .SDAT files. That is, separation of 
real and imaginary components and arranging the data on a 3D or 4D hyper-matrix (depending if it is a 2D or 3D MRSI study respectively).

- Reading multi-coil MRSI data in .7 files and coil signals combination

The reading procedure for the .7 multicoil files $\left[\mathrm{REH}^{+} 90\right]$ is the same as the one used for single coil. However, the file is $n$ times larger than the single coil, where $n$ is the number of coils used. The information of each coil is concatenated. That is, firstly we read the information obtained by the first coil, secondly the information of the second coil and so on until the end of the file.

Once the MRSI data of each coil is read, we have to combine it to obtain a single k-space to work with. To do so we use the signal combination algorithm for optimal SNR defined by Wright et. al. in [WW97] used in several studies. It consists on the combination of spectra from different coils by simply adding the weighted spectra on a point-by-point basis to obtain a single SNR optimized spectra:

$$
S_{k m}^{c}=\sum_{j=1}^{N} w_{j k} S_{j k m}=\left[w_{k}\right]^{\top}\left[S_{k m}\right]
$$

where $S_{\mathrm{km}}^{c}$ is the combined spectra, $S_{j k m}$ is the $m$ th spectral point in the kth spectra from coil $j$, and $w_{j k}$ is the weighting coefficient for the $k$ th spectra from coil $j$. For chemical- shift imaging, $k$ would run from 1 to $N_{\text {voxels }}$, the number of voxels. Equation 49 assumes a voxel size smaller than the rate of variation of the flux density of individual coils and therefore that the weighting functions derived are common to all spectral points.

The relative height of a particular spectral peak or linear combination of peaks can be used as an approximation to the flux density magnitudes at the location of the voxel from each coil. Therefore we can use the height of the water peak relative to the signal intensity at each voxel, as an approximation of the weighting function for this voxel. Using this approximation and denoting the reference peak spectral point as $m_{\text {ref }}$, the weighting functions for the $j_{t h}$ coil and the $k_{t h}$ voxel become:

$$
\left[w_{k}\right]=\frac{\left[S_{k_{\text {ref }}}\right]}{\left\|S_{k m_{r e f}}\right\|}
$$

And therefore using Equations 49 and 50, it is obtained the equation for SNR weighted spectra:

$$
S_{k m}^{c}=\frac{\left[S_{k m_{r e f}}\right]\left[S_{k m}\right]}{\left\|S_{k m_{r e f}}\right\|}=\frac{\sum_{j=1}^{N_{\text {coils }}}\left(S_{j k m_{r e f}} S_{j k m}\right)}{\sqrt{\sum_{j=1}^{N} N_{\text {coils }}\left(S_{j k m_{r e f}}\right)^{2}}}
$$



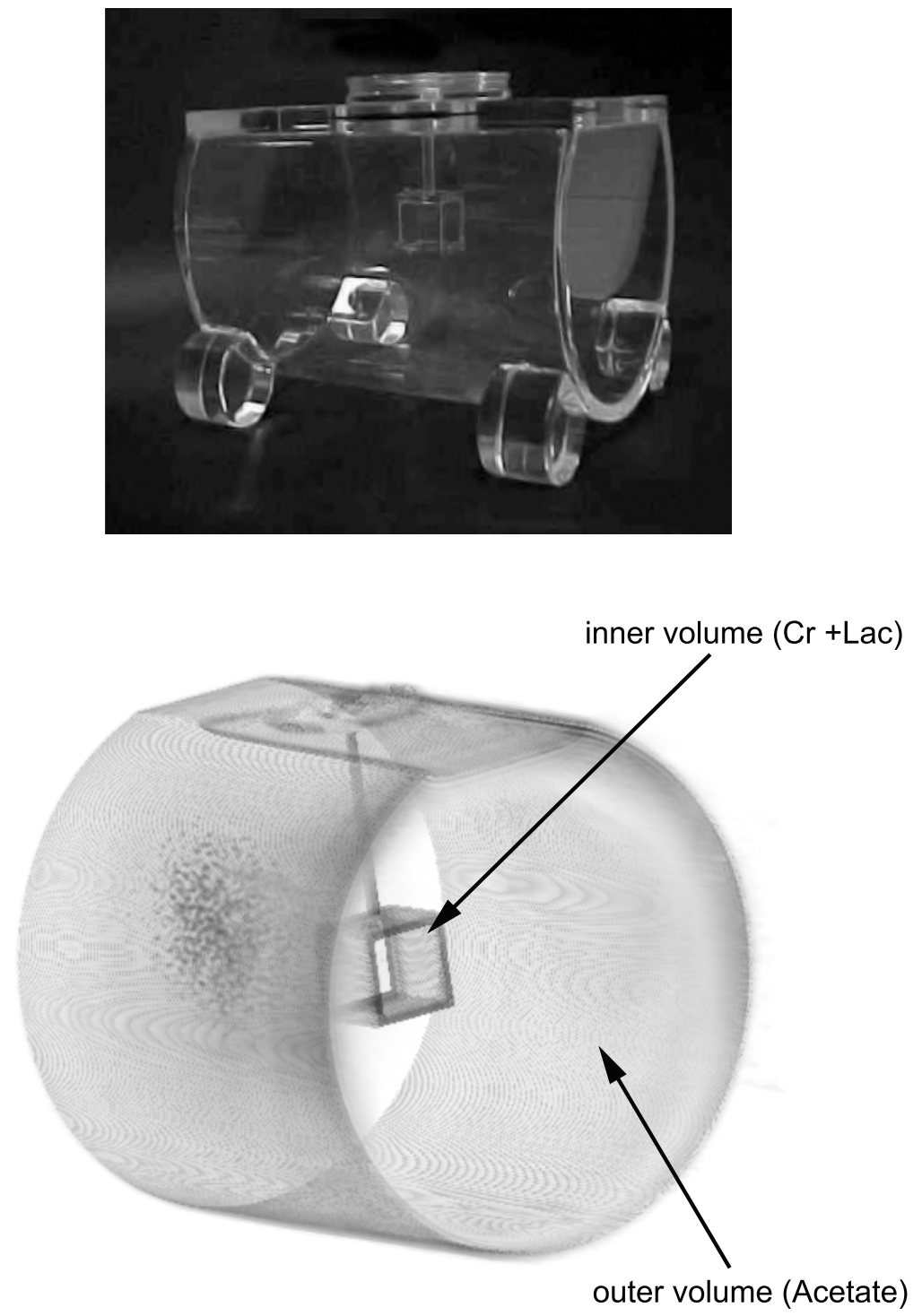

Figure 27: MRSI cylindrical phantom (top) and the 3D render of the phantom obtained from 2D MRI images (bottom). The phantom contains $\mathrm{Cr}$ and Lac in the inner cubic volume and acetate in the outer volume, thus allowing assessment of the efficiency of acquiring a spectrum from a localised region and the rejection (contamination) of signals from regions outside of the selected volume 


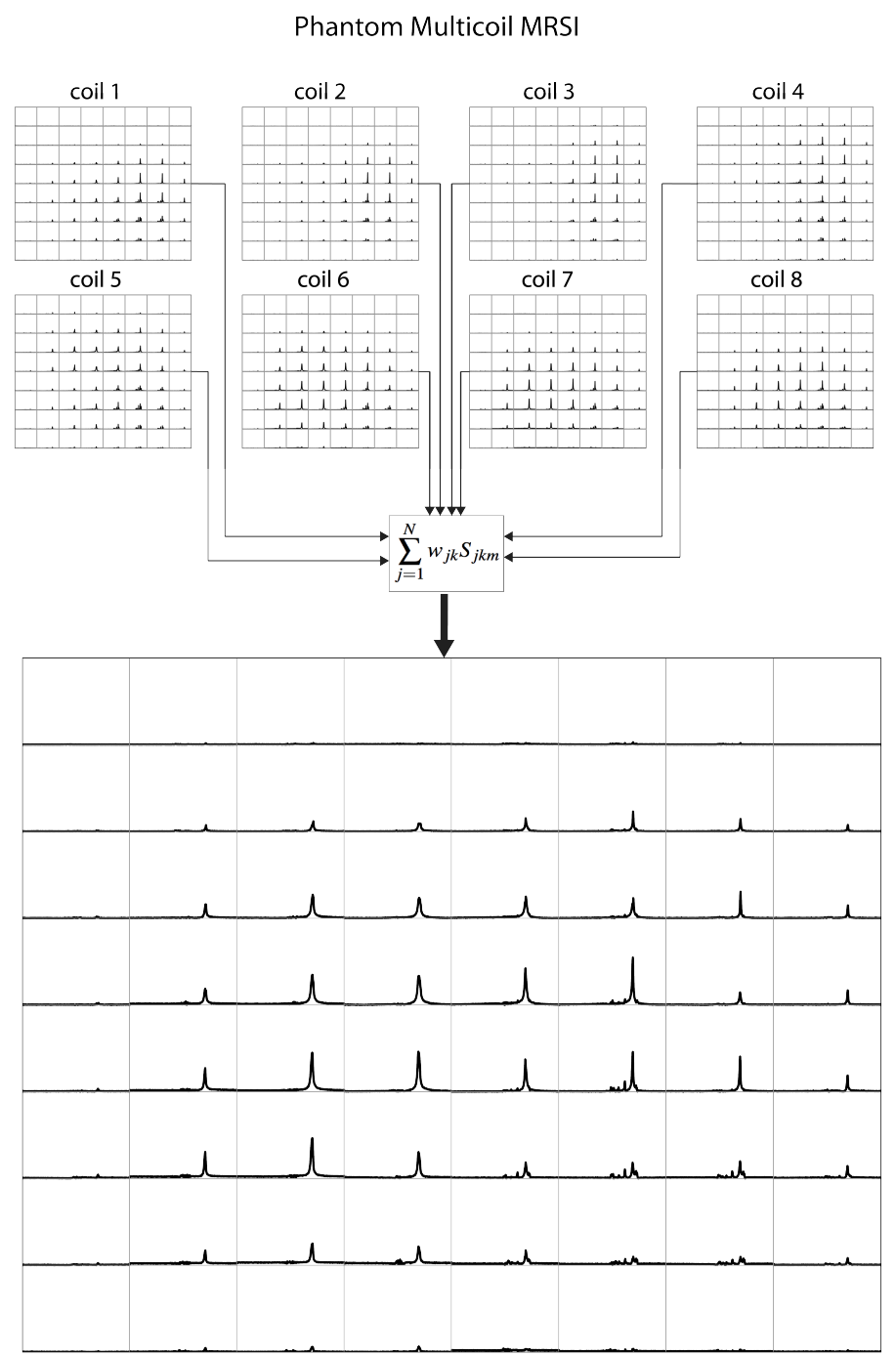

Figure 28: Multi-coil MRSI combination by the algorithm for optimal SNR. 
To test the reconstruction algorithm for multi-coil MRSI data combination an experimental test acquired in St George's Hospital was used. It consists in the pre-processing and analysis of the MRI-MRSI data obtained from a cylindrical phantom (see Figure 27 top). The phantom, a $2 \mathrm{~cm}$ cube within a $14 \mathrm{~cm}$ diameter cylinder, still seems a practical design. The phantom contains $\mathrm{Cr}$ and Lac in the inner cubic volume and acetate in the outer volume, thus allowing assessment of the efficiency of acquiring a spectrum from a localized region and the rejection (contamination) of signals from regions outside of the selected volume.

The MRI-MRSI data were obtained with a 1.5T GE Signa HDxt MR scanner using a 8HRBRAIN multicoil. The MRI acquisition protocols included the following parameters: T2-PD sequence (TE of $89.4 \mathrm{~ms}$ and TR of $3600 \mathrm{~ms}$ ), slice thickness of $5 \mathrm{~mm}$, FOV of $912 \times 497 \mathrm{~mm}$, and image size of $512 \times 512$. The MRSI acquisition protocols included PROBE-P sequence with the following spectral parameters: TR of $1000 \mathrm{~ms}$, TE of $144 \mathrm{~ms}, 8 \times 8 \times 8$ voxels, 1024 points and a FOV of $120 \times 120 \times 120 \mathrm{~mm}$. A 3D reconstruction of the phantom volume, have been done using OSIRIX software, and is presented in Figure 27 (bottom).

A MRI-MRSI exam had been performed to test the co-registration between both modalities and to identify the voxel with the phantom solution. The results obtained for each coil and the combination of them by the use of the algorithm for optimal SNR can be seen on Figure 28.

\subsubsection{MRSI preprocessing module}

The MRSI preprocessing module includes a fully automatic processing pipeline based on different studies published over the last few years [SJB08, LPS05, $\mathrm{SMdE}^{+}$05]. This pipeline could be divided into two main parts: the $\mathrm{K}$-space processing and the processing over the FID's.

\section{$K$-space preprocessing}

The K-space preprocessing consists in three main steps: K-space weighting, zero-filling, and 2D or 3D Fourier transform.

- K-space weighting or apodization

The aim of K-space weighting is to modify the Point Spread Function (PSF) according of each specific application. The PSF represents the response of the image reconstruction system for a point source, and therefore is relative to the efficiency of the reconstruction method. Multiplying the K-space by a window function, the PSF can be modified. This is because the Fourier transform of the product of two functions is equivalent to the convolution of the transform of each of the 
functions. The application of this window function (also known as apodization function) to the K-space is known as K-space apodization. The apodization presents a compromise between the PSF side lobe reduction and the principal lobe amplitude, that is a compromise between spatial resolution and SNR.

In this pipeline the three mostly used filters for apodization have been implemented:

\section{Cosine filter}

$$
w(n)=\cos \left(\frac{2 \pi n}{N}\right), 0 \leqslant n \leqslant N
$$

Hanning filter. The Hann and Hamming windows, both of which are in the family known as "raised cosine" or "generalized Hamming" windows, are respectively named after Julius von Hann and Richard Hamming.

$$
w(n)=0.5-0.5 \cos \left(\frac{2 \pi n}{N}\right), 0 \leqslant n \leqslant N
$$

Hamming filter. The Hamming filter is considered as optimum for most of MRSI applications [LPS05, EBW87]. The "raised cosine" with these particular coefficients was proposed by Richard W. Hamming. The window is optimized to minimize the maximum (nearest) side lobe, giving it a height of about one-fifth that of the Hann window.

$$
w(n)=0.54-0.46 \cos \left(\frac{2 \pi n}{N}\right), 0 \leqslant n \leqslant N
$$

For simplicity Equations 52, 53, and 54 are referred to 1D versions of these apodization filters, it is easy to generalize them for higher dimensions.

In Figure 29 the shape of each of the apodization functions and the respective PSF are presented for the case of 1D and 2D.

- K-space zero-filling

Zero-filling can be considered as an interpolation technique based on the Fourier analysis properties. Zero-filling consist on adding zeros at the edges of the K-space. Increasing the dimensionality of the K-space by adding zeros, we also increase the dimensionality of the MRSI and therefore the number of the voxels. To perform the zero filling, the apodization of the $\mathrm{K}$-space is mandatory. The apodization ensures that the values at the edges of the K-space are close to zero. If the K-space is not apodized, when adding zeros at the edges, some discontinuities could be introduced in the K-space and some artifacts will appear in the reconstructed MRSI. 


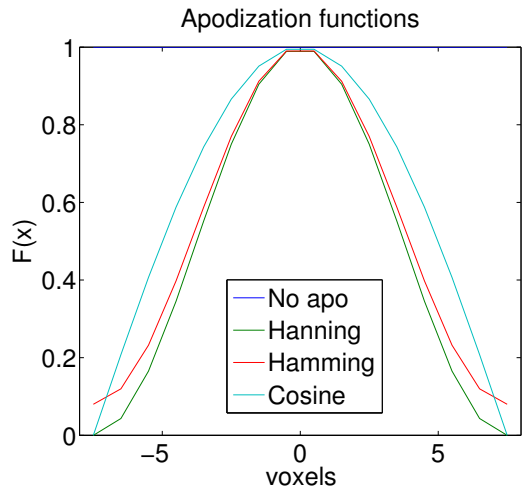

No apodization function

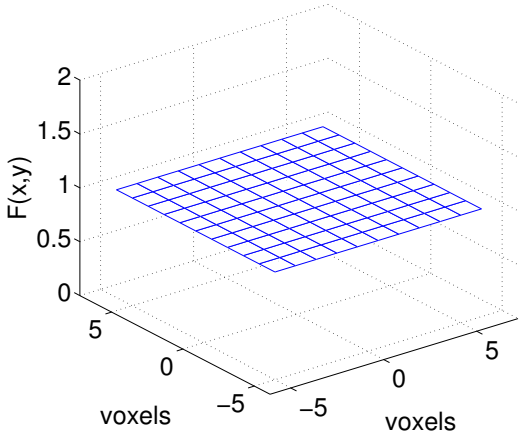

Cosine apodization function

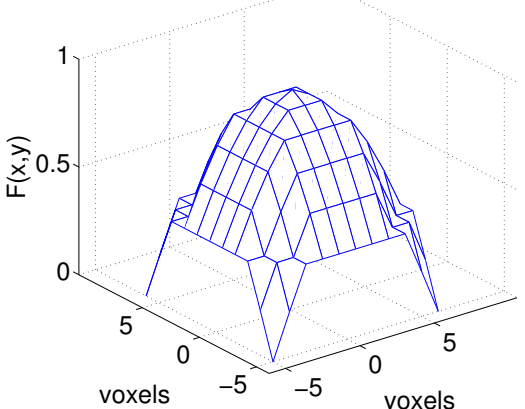

Hanning apodization function

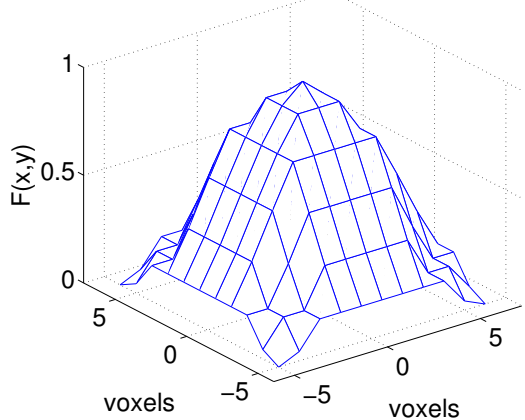

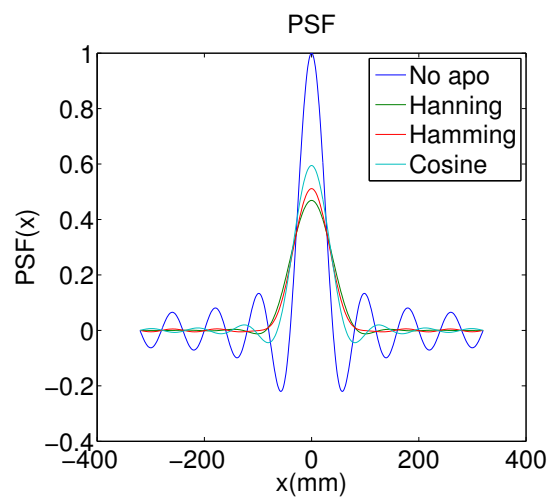

No apodization PSF

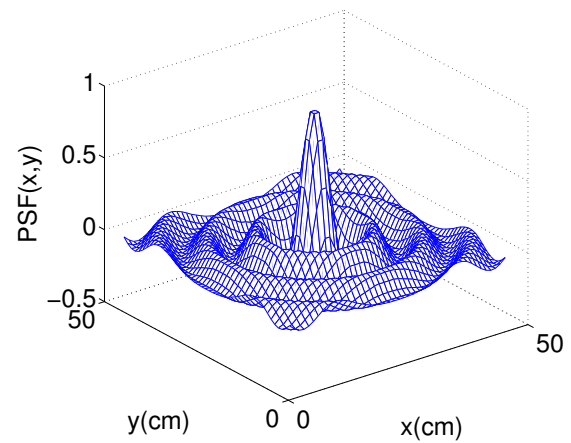

Cosine PSF

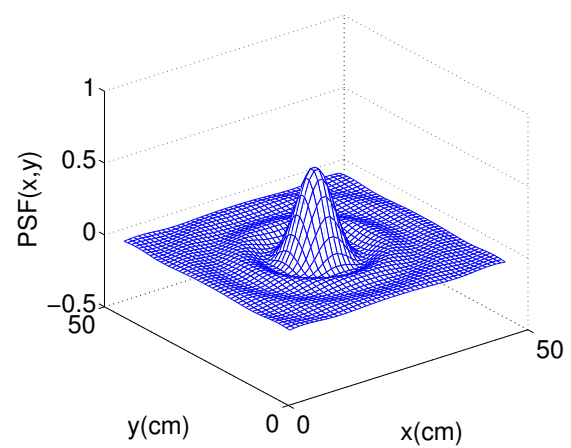

Hamming PSF

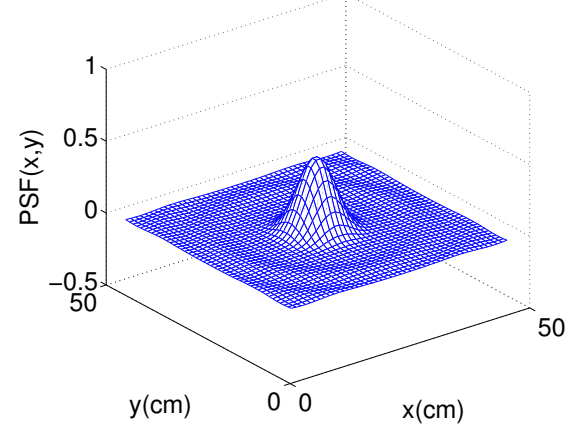

Figure 29: K-space apodization functions (left) and their respective PSF (rigth). From top to bottom: a comparison of the different apodization functions used in MRSILab and their respective PSF; the PSF without apodization; the PSF without apodization; 


\section{- K-Space Fast Fourier Transform (FFT)}

Once the $\mathrm{K}$-space is processed we have to compute the $\mathrm{n}$-dimensional FFT of the K-pace to obtain the MRSI. This transform are applied over all dimensions except the temporal one. After the FFT transform was applied we obtain a hyper-matrix $M$ with the intensity for each voxel at each time $t$, that is a FID signal for each voxel (for a detailed description see Subsection 2.2.6.

\subsubsection{FID preprocessing module}

The FID's obtained by the k-space FFT can be processed using the same pipeline applied to SVS. In MRSILAb a basic pipeline consisting on the following steps were implemented: Eddy Current Correction (ECC) based on the water unsuppressed FID, water suppression based on Hankel-Lanczos singular value decomposition (HLSVD) algorithm, apodization, and baseline offset correction. A more detailed description of each of these processing steps could be seen in Section 3.2.

\subsubsection{MRI-MRSI co-registration module}

The MRI-MRSI co-registration module is responsible for matching both imaging modalities in the same coordinate system. In addition, this module seeks the MRI images that coincide in same space as each of the MRSI slices. This allows us to superimpose the anatomical information provided by MRI and the metabolomics information provided by MRSI. The co-registration module is a generalization of the the work and algorithms of Montellius in [Mon08] for the case of MRSI. In this work Motellius presents a coregistration module for MRI and SV-MRS, from an experimental setup based on MRI-MRS phantom scans. At present, the MRI-MRSI co-registration module works with Philips cases and is being developed to be compatible with General Electric cases.

\section{Creation of VOI coordinates}

Firstly we need to extract the MRI coordinates defined in the DICOM headers, using the specifications defined by the standard and widely defined in the literature (see [Ass00]). These variables are defined on Table 5 as they were documented in [Mon08].

Secondly, as was described on Section 3.1.3, we obtain the MRSI coordinates from the data contained on .SPAR file, assigning a coordinate to each FOV corner.

Finally, we transform the MRSI coordinates to the coordinate system defined for the MRI DICOM images. To do so, a transformation must be applied 
Table 5: Position variables obtained from DICOM MRI images and a brief description.

\begin{tabular}{|c|c|}
\hline Variables defined & Description \\
\hline $\bar{p}$ & $\begin{array}{l}\text { The ImagePositionPatient tag, revealing the image coordinates } \\
\text { of the centre of the upper leftmost pixel in an image, was imported } \\
\text { from each image in the stack and stored in the kth entry of the } \\
\text { column vector corresponding to image number } k \text {. }\end{array}$ \\
\hline $\bar{n} 2$ & $\begin{array}{l}\text { A vector, equal in dimensions as } \mathrm{p} \text {, but with the DICOM } \\
\text { filename stored in each entry. }\end{array}$ \\
\hline$n$ & $\begin{array}{l}\text { Number of columns (pixels in j-direction) in the images, } \\
\text { imported from the Width tag. }\end{array}$ \\
\hline$n 1$ & $\begin{array}{l}\text { Number of rows (pixels in the } i \text {-direction) in the images, } \\
\text { imported from the Height tag. }\end{array}$ \\
\hline$\overline{\mathrm{r}}$ & $\begin{array}{l}\text { The unit vector (Euler angles) defined by any row of pixels } \\
\text { in an image, imported from the ImageOrientationPatient tag. }\end{array}$ \\
\hline$\overline{\mathrm{c}}$ & $\begin{array}{l}\text { The unit vector (Euler angles) defined by any column of pixels in } \\
\text { an image, imported from the ImageOrientationPatient tag. }\end{array}$ \\
\hline$p s_{r}$ & $\begin{array}{l}\text { Spacing between pixels in a row, imported from the } \\
\text { PixelSpacing tag. }\end{array}$ \\
\hline$p s_{c}$ & $\begin{array}{l}\text { Spacing between pixels in a column, imported from the } \\
\text { PixelSpacing tag. }\end{array}$ \\
\hline
\end{tabular}


to the MRSI coordinate system defined by three rotational matrices and one translational matrix.

\section{Intersection}

Once both image modalities are referenced to the same coordinate system, we need to identify the MRI images included in each MRSI slide FOV. To do so, we obtain each MRI plane defined by three of their corners. Later, we obtain the lines that connect each of the 8 corners of the each MRSI slice, and we look for an intersection between the MRI planes and the lines defined for each MRSI slice. In case of intersection we associate this image to the MRSI slice for superimposed display on the GUI visualization module.

\subsubsection{Quantification module}

The MRS quantification allows us to estimate the relative or absolute metabolite concentration in a sample by means of the MRS information. This analysis facilitates the interpretability of the information contained in the MRSI by means of metabolite concentration maps.

In the current version of MRSIlab the HLSVD algorithm was included to quantify the MRSI spectra. The implementation of the algorithm used was developed by the Katholieke Universiteit Leuven. Although there are more sophisticated techniques for quantification, HLSVD gives us enough precision in its results, it does not need to much prior information and is also needed for the water peak suppression.

\subsubsection{The graphical user interface}

The GUI of MRSIlab has been developed using the MATLAB Graphical User Interface Development Environment (GUIDE). The Main window and the menu for loading the NMR (MRI and MRSI) data into the system are described below.

\section{Loading NMR data menu}

The loading MR data menu (Figure 30) allows to specify the MRI and MRSI files to load as well to define some parameters for the data processing as can be the K-space apodization function, the use of water reference file for the MRS Eddy Current Correction. 


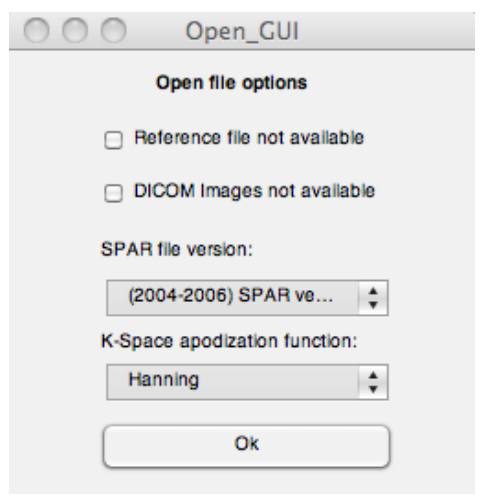

Figure 30: MRSIlab data loading window.

\section{Main window}

The main window of the software presented can be seen in Figure 31. In this window four regions are clearly defined attending to their functionality: An MRI-MRSI plot, patient information, spectra plot and nosological images plot.

- MRI-MRSI plot: In this plot the MRSI grid position is superimposed to the corresponding MRI anatomical image obtained in the co-registration step. This plot allows us to obtain clinical information from the MRI and to select the voxels for spectra visualization in the spectra plot.

- Patient information: In this main window region clinical patient information included in the DICOM and SPAR/SHF files are shown.

- Spectra plot: In this plot the spectra selected over the MRSI grid of the MRI-MRSI plot can be shown. The spectra plot allows to plot of superimposed spectra and the representation of the quantification results.

- Nosological image plot: The last region is dedicated to plot quantification or nosological images over the MRI image. Different options can be selected to choose between quantification maps corresponding to different metabolites or nosological images obtained from linear combination of metabolite concentrations. This option allows us to incorporate widely used rules to evaluate tumour probability in prostate analysis, simple classification models for risk evaluation, or classification tumour models based on PR..

\section{Additional Functionalities}

In addition to the functionalities for MRSI processing and visualization described above, the implemented software contains some useful additional tools for MRI-MRSI analysis. 


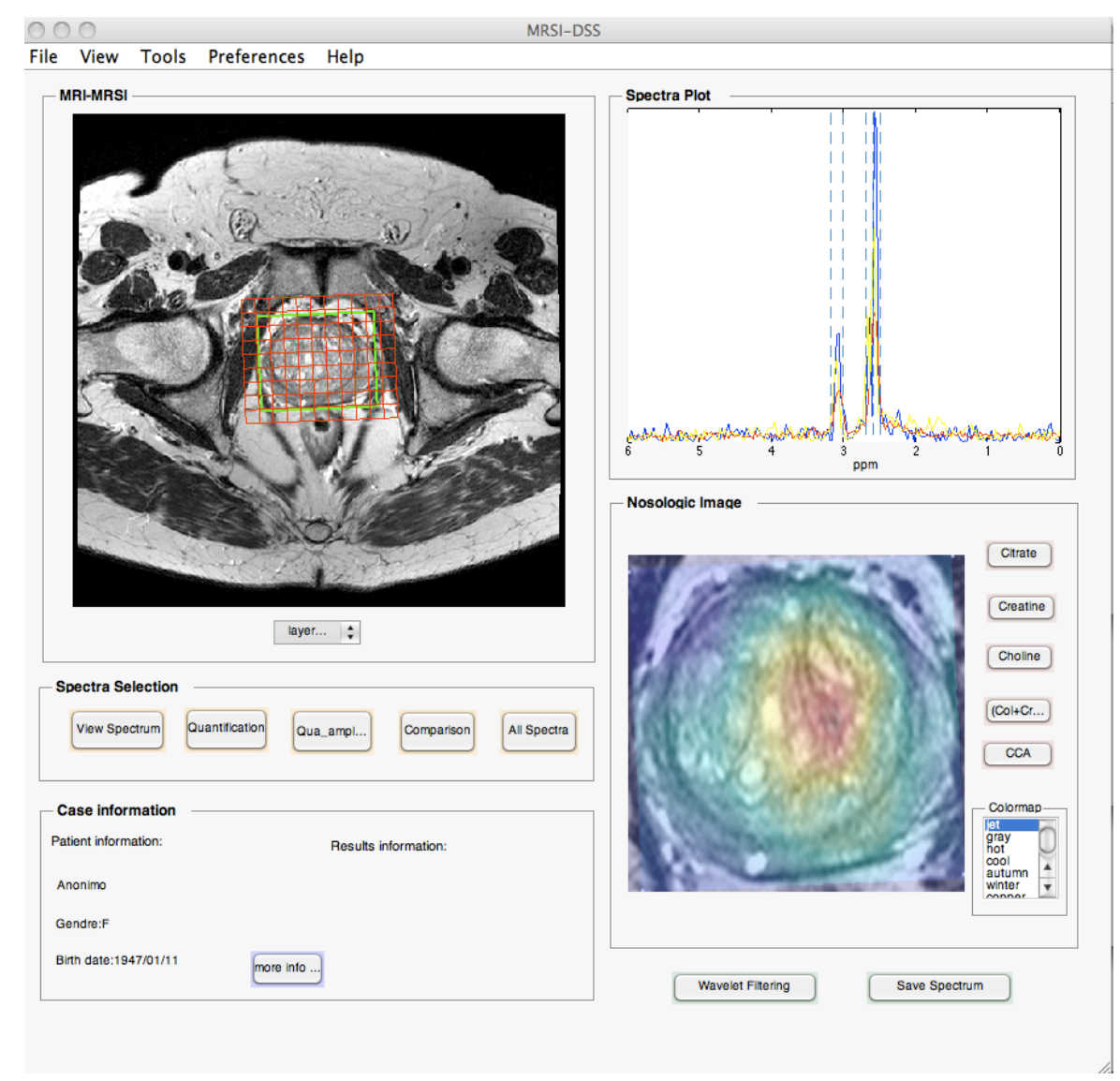

Figure 31: MRSIlab main window 
- DICOM anonymization: This module allows us to anonymize the DICOM files patient information, deleting the DICOM fields related to non-clinically relevant patient information.

- DICOM splitter: This module allows to convert a multi-frame DICOM file into several single-frame DICOM files compatible with the software developed.

- SPAR anonymization:This module allows us to anonymize the SPAR files patient information, deleting the SPAR fields related to nonclinically relevant patient information.

\subsubsection{Application}

MRSILab is being tested in the context of prostate tumors and brain tumors. For evaluating the MRSILab toolbox in the case of prostate tumours, data collected over the DSSPROSTATA (FIT-340001-2007-14) and DSSPROSTATA2 (TSI-020302-2009-65) projects have been used. These data include include cases of $1.5 \mathrm{~T}$ and $3 \mathrm{~T}$ MRSI. Moreover, in order to evaluate the MRSILab toolbox in the case of brain tumours, data collected over the eTumour (LSHC-CT-2004-503094) and HealthAgents (ST-200427214) EU projects have been used. 


\subsection{DESIGN AND IMPLEMENTATION OF A SV-MRS AUTOMATIC PRE-PROCESSING PIPELINE FOR CURIAM-BT CDSS}

\subsubsection{Motivation}

CURIAM is a generic CDSS. It can incorporate multiple predictive models based on biomedical observations to allow the extraction of knowledge about new cases, offering specific domain guided visualization to medical specialists $\left[\mathrm{SGGV}^{+}\right.$09]. The main benefits and applications of CURIAM include the quickly expert diagnosis validation, support on treatment planning, assistance in the training of health personnel, extraction of biomedical indicators of significant pathology, and detection of rare cases.

CURIAM is designed to work with most types of biomedical data, to support any real medical problem described by such data, and to be constantly updated. It also offers a scalable architecture that allows the immediate incorporation of new predictive models or classifiers through definitions in XML. CURIAM supports the analysis, processing, and sorting new patients based on numerical data, categorical data or biomedical signals .

Currently we have developed the following versions of CURIAM:

- CURIAM-BT: CDSS for the diagnosis of brain tumors with magnetic resonance spectroscopy.

- CURIAM-STT: CDSS for the diagnosis of soft tissue tumors by MRI findings

- CURIAM-PPD: CDSS for the early detection of postpartum depression.

In this section we will focus on CURIAM-BT. Currently, the diagnosis gold standard of brain tumours is biopsy and subsequent histopathological analysis of the sample of brain tissue (ex-vivo method). This process, however, is highly invasive and can even cause death. The safe and noninvasive alternative is to obtain in-vivo diagnosis, e.g. through magnetic resonance imaging. The objective of CURIAM-BT is to contribute to the robust diagnosis of brain tumours based on SV-MRS data. To do so, data analysis techniques of magnetic resonance with machine learning methods have been developed and implemented, paying particular attention to evaluation to obtain robust classification results. A CURIAM-BT screenshot is presented in Figure 32.

CURIAM BT has a strong research component, however, at the Hospital Universitario Doctor Peset and Hospital de la Ribera, both in Valencia, Spain, an evaluation project to determine that it is also a useful tool for the clinical setting is being conducted. As a result of this evaluation, CURIAM BT will be deployed in these hospitals and the Clínica Quirón in Valencia.

In CURIAM-BT the development and implementation of a robust semiautomatic SV-MRS pre-processing pipeline is mandatory. In the following 


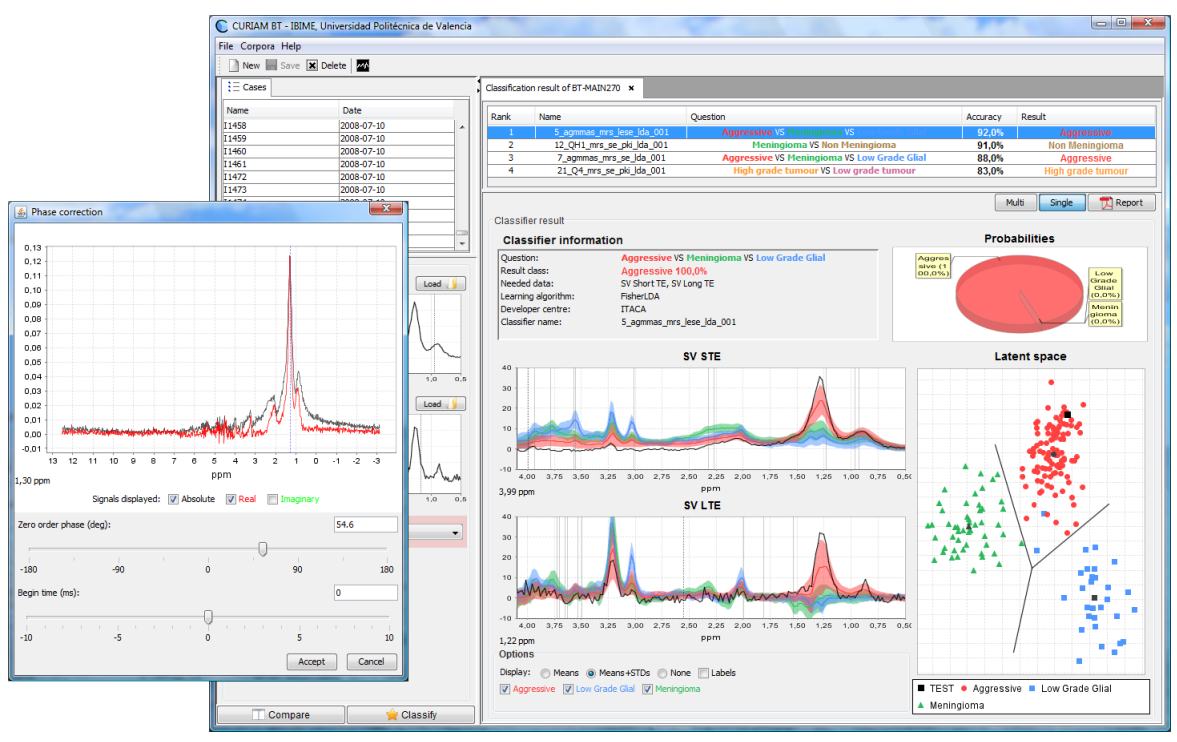

Figure 32: CURIAM-BT screenshot.

section we will introduce the pipeline designed for the current version of CURIAM-B, partially based on the protocol defined throughout the INTERPRET [INT08]. With the development of CURIAM BT we have achieved the automatization of the main part of the MRS pre-processing. Moreover a manual phase correction module in Java has been designed. Finally, the results of CURIAM BT (including the pre-processing pipeline) were evaluated for both 1.5 and 3T MRS spectra in different hospitals $\left[\mathrm{SGGAB}^{+}\right.$12].

\subsubsection{SV-MRS pre-processing pipeline}

In CURIAM-BT, the FID loaded is preprocessed following a well-established MRS data pre-processing protocol and quality control. This protocol was developed during the INTERPRET project [INT08] and was also adopted by the EC project, contract no. FP6-2002-LIFESCIHEALTH 503094 (eTUMOUR) project [The07] in order to guarantee the coherence in PR studies and Decision Support System [GGVFRV05, GGVRRV05].

Figure 33 shows the MRS data pre-processing protocol applied to all the spectra loaded in CURIAM-BT. The inputs of the procedure are the watersuppressed and the water unsuppressed FID signals for each acquisition (Short TE and Long TE).

The input signals of the preprocessing pipeline are the FID, and the FID unsuppressed signal as water-reference (see Figure 34).

The steps carried out for the MRS data pre-processing protocol for each spectrum were: 


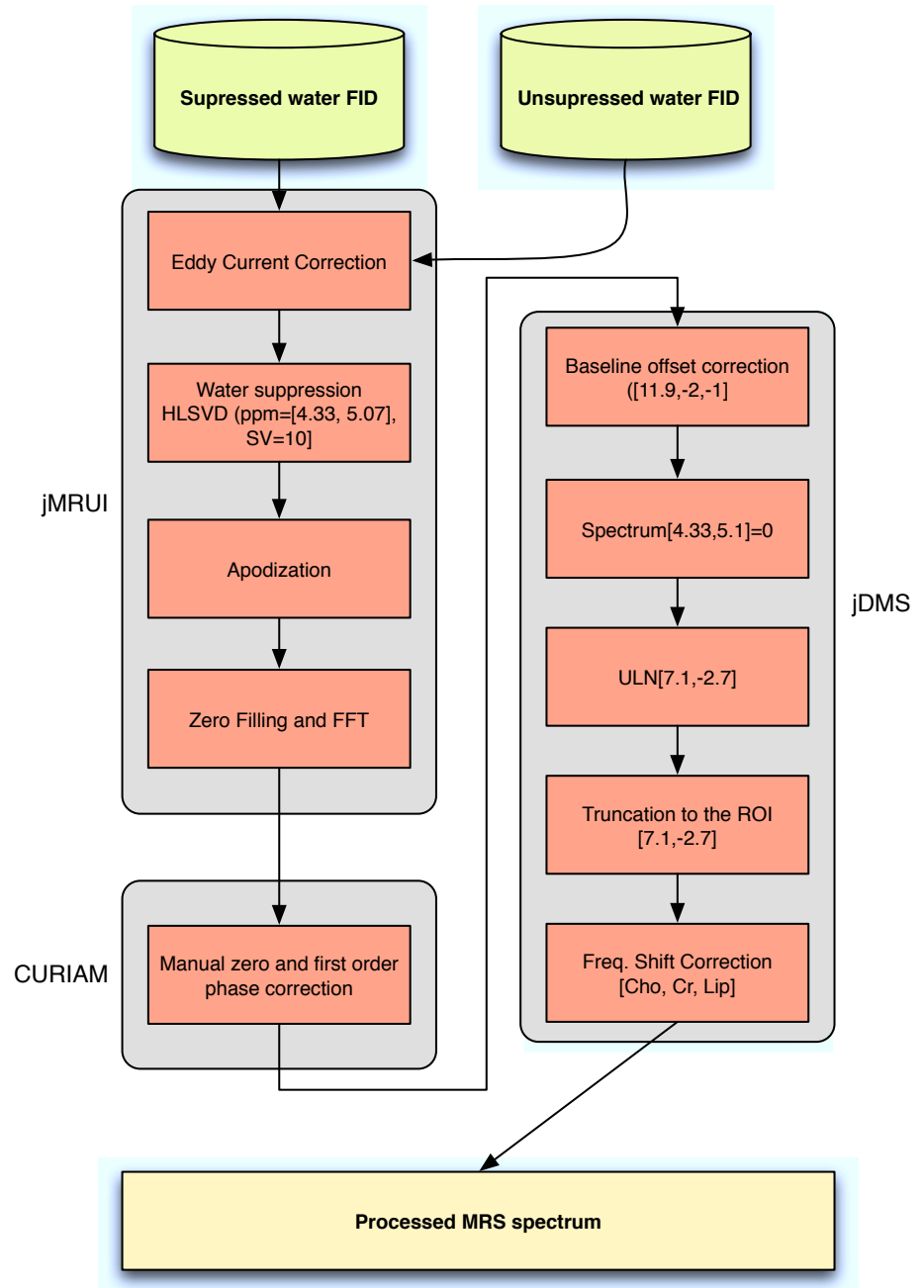

Figure 33: CURIAM MRS data pre-processing pipeline. 

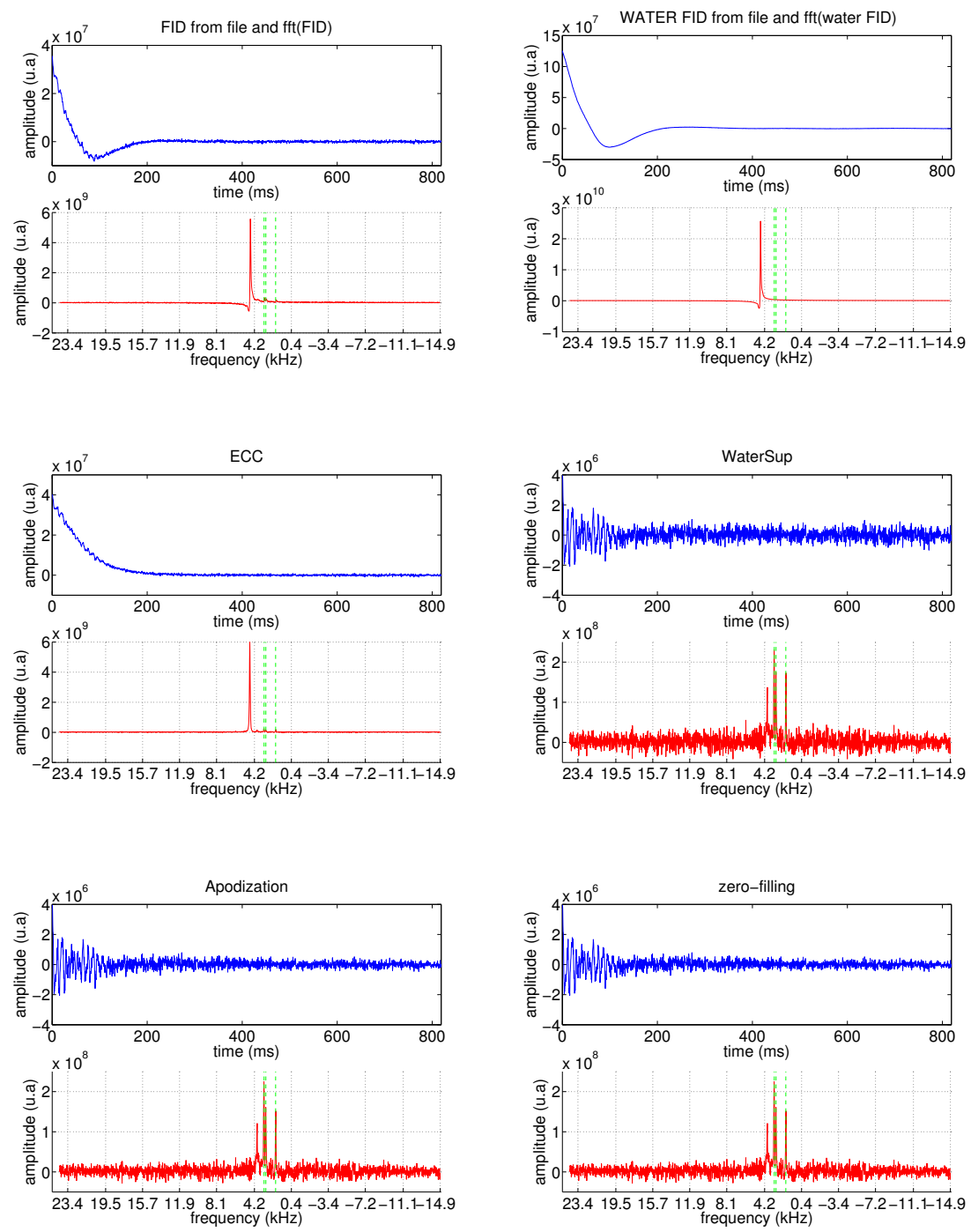

Figure 34: Real part of the FID signal and real part of the Fourier transform of: the FID signal, FID unsuppressed water signal, Eddy current corrected signal, residual-water suppressed signal, and apodized signal. 

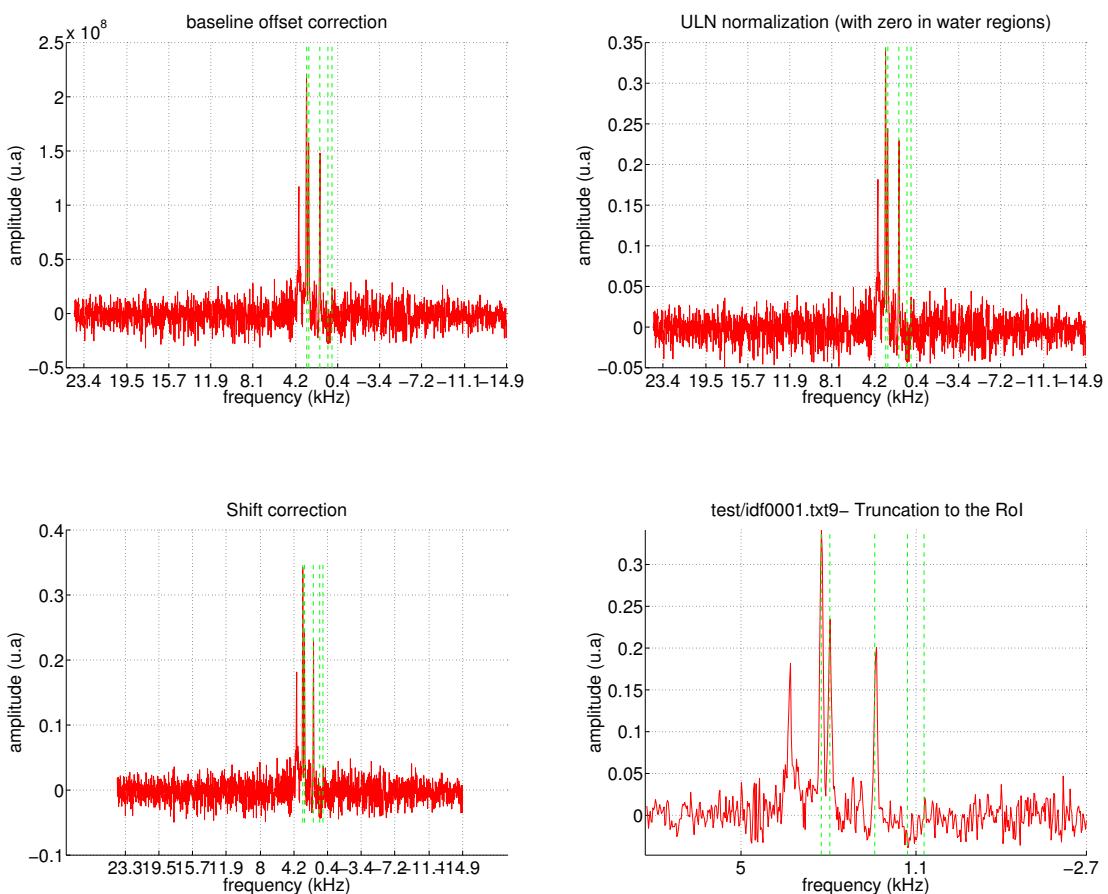

Figure 35: MRS signal after: baseline offset correction, ULN, frequency shift correction, and truncation to the ROI.

1. The water-suppressed FID was corrected for eddy current effects using the deconvolution of metabolite FID with its water FID by the QUALITY algorithm [dGvDB90]. Figure 34 shows the result of this step in the original FID signal.

2. HLSVD algorithm $\left[\mathrm{CCGLF}^{+} 01\right]$ was applied for fitting and removing the residual water resonance. The filter was parametrized with 10 singular values and limited to the ppm range $[4.33,5.07] \mathrm{ppm}$. Figure 34 shows the result of this step.

3. Apodization is applied to improve the Signal-to-Noise Ratio (SNR) of the spectrum [Gad95]. The time domain signal is multiplied point-bypoint by a decaying exponential function to lend the most weight to the initial part of the FID, and then attenuate the contribution of the latter part, where the SNR is lower. The apodization applied was a Lorentzian with a line broadening echo of $1 \mathrm{~Hz}$. Figure 34 shows the result of this step.

4. The FFT, with zero-filling up is applied to obtain the frequency domain spectrum. The zero-filling [Gad95] could be considered as an interpolation method that allows us to equal the spectra number of points to 2048 .

5. A manual phase correction is applied to correct zero (non-frequeny dependent) and first order (proportional to the frequeny) phase distortions. 
6. The baseline offset is subtracted to every point of the spectrum $S$. The baseline offset is estimated previously as the mean of the $\mathrm{N}$ values $S\left(f_{n}\right)$ in the ranges $[11,9] p p m$ and $[-2,-1] p p m$ (equation 55).

$$
S(f)_{b o}=S(f)-\frac{1}{N} \sum_{n=1}^{N} S\left(f_{n}\right)
$$

Figure 35 shows the result of this step.

7. The region $[4.33,5.1] \mathrm{ppm}$ is Short TE to zero to avoid the influence of the residual water peak.

8. ULN: The spectrum is normalized setting the region of interest $[7.1,-2.7] p p m$ to unit length by the equation 56 .

$$
S(f)_{\text {norm }}=\frac{S(f)}{\sqrt{\sum_{f^{\prime}=[7.1,-2.7]} S\left(f^{\prime}\right)^{2}}} * 1000
$$

Figure 35 shows the result of this step.

9. A shifting correction is applied [PROCJS ${ }^{+} 08$ ] to align the spectrum searching, in this order, for the relative positions of the $\mathrm{Cr}$, Cho L1 peaks at $3.03,3.21$, and $1.29 \mathrm{ppm}$ respectively. Each peak is searched in a range near the expected position $\pm 0.04 \mathrm{ppm}$. If none of these peaks is found the spectrum is left unchanged.

Figure 35 shows the result of this step.

10. The spectrum is truncated to the region of interest $[4.3,0.1] \mathrm{ppm}$. A truncation to the typical region of interest is shown in Figure 35.

In order to implement the first four steps [1 -4] the jMRUI package $\left[\mathrm{VVV}^{+} 96\right]$ in batch mode was used. The manual phasing (step 5) was implemented in Java. Finally for the last steps $[6-10]$ the jDMS package [PROCJS ${ }^{+}$08] in batch mode was used. 


\subsection{DESIGN AND IMPLEMENTATION OF ARRHYTHLAB: A SOFT- WARE FOR ECG PROCESSING, QUALITY CONTROL, SEGMEN- TATION, AND BEAT LABELING.}

\subsubsection{Motivation}

The electrocardiography provides information about the heart's internal electrical system. This information is very useful for heart disease diagnosis and, more specifically, it is crucial to understand and identify arrhythmias.

Unlike other cardiac pathologies, arrhythmias do not show a fixed pulse pattern, but different morphologies of pulses in a single ECG exam. Therefore in order to report a detailed diagnostic of an arrhythmia episode, the annotation of the pulses, assigning a diagnostic label or labels to each pulse, would be desirable. However this annotation would need to be done by expert cardiologists and would be very time consuming.

To simplify this process a software for electrocardiograms beat labeling Arrhythlab has been designed and implemented. Up to our knowledge there is not any available software capable of processing ECG signal and detecting QRS complexes, and that it allows manual labeling of each of the detected beats. Arrhythlab allows the automatic pre-processing of the ECGs, the segmentation of the pulses included on it, the edition of the incorrect segmentation results by adding or removing detected pulses, and label each of the ECG beats by adding one or more tags according to diagnosis. The results of the segmentation and annotation process can be saved to a file for future reference and for the sharing of results. In turn, the results can be opened directly exported from MATLAB, which simplifies the translation of the results to a working environment and their subsequent use in scientific studies. In the design and implementation has made prioritizing ease of use, functionality, implementation in major operating systems (Mac / Windows) and accessibility to download it free via the web to make this program a potentially useful tool both for scientific studies more complex for use in daily clinical practice.

Arrhythlab helps in the compilation of large arrhythmia ECG datasets reducing the time cost of beat labeling. The detailed information obtained with Arrhythlab plus the automatic preprocessing, quality control, and segmentation pipeline of each ECG record makes possible the design and training of supervised classification models, as well as it contributes to a fast development of decision support systems for arrhythmias based on ECG morphologies. 


\subsubsection{Pre-processing}

The ECG signal needs to be processed before using it for diagnostic purposes. In Arrhythlab a set of filters were defined to remove common artifacts in ECG.

Firstly a high pass filter was designed to remove baseline artifacts. Low frequency artifacts and baseline drift may be caused in patient movements during the ECG acquisition. Moreover, poor contact and polarization of the electrodes contribute to the baseline distortion. The filter designed to remove baseline artifacts was high pass windowed linear-phase FIR digital filter defined by a normalized cutoff frequency $\mathrm{Wn}=0.5 / \mathrm{Nq}=10^{-} 3$ (where $\mathrm{Nq}$ refers to Nyquist frequency), and 1501 coefficients. Their magnitude response can be seen in Figure 36 (top).

Secondly a low pass filter was designed to remove high frequency noise in the ECG. The high frequency noise cold be due to the instrumentation amplifiers or the recording system. The filter designed to remove high frequency noise was defined with a high frequency pass at $45 \mathrm{~Hz}$, and the frequency-domain transfer function presented on equation 57 . Their magnitude response can be seen in Figure 36 (middle).

$$
\mathrm{H}(z)=\frac{1+z^{-1}}{8.0252-6.0257 \cdot z^{-1}}
$$

Finally a band cut filter was designed to remove power line interference at $50 \mathrm{~Hz}$. The filter used was a Parks-McClellan FIR filter [Com79] of 254 order and centered in $50 \mathrm{~Hz}$. Their magnitude response can be seen in Figure 36 (bottom).

\subsubsection{QRS detection and segmentation}

For the detection of $\mathrm{R}$ wave, a batch QRS detector was used based upon that of Pan, Hamilton and Tompkins [HT86] and implemented in MATLAB by G. Clifford. Once the temporal points $t_{i}$ associated to each $R$ wave $R_{i}$ along the ECG exam E are known, each beat $i$ is segmented considering the length of each beat as the temporal interval between $t_{i}-200$ and $t_{i}+200$. That is each beat $B_{i, l}$ is defined as $B_{i, l}(t)=E_{l}\left(\left[t_{i}-0.2 s, t_{i}+0.2 s\right]\right)$, where $l$ refers to each lead used in the ECG record.

\subsubsection{Beat editing and labeling}

Arrhythlab allows the clinician to manually edit the automatically detected beats. By clicking on a specific beat in the ECG plot in the main window, the user can delete an incorrectly detected beat or introduce a new beat not detected by the algorithm. Moreover, in case of high number of incorrectly 

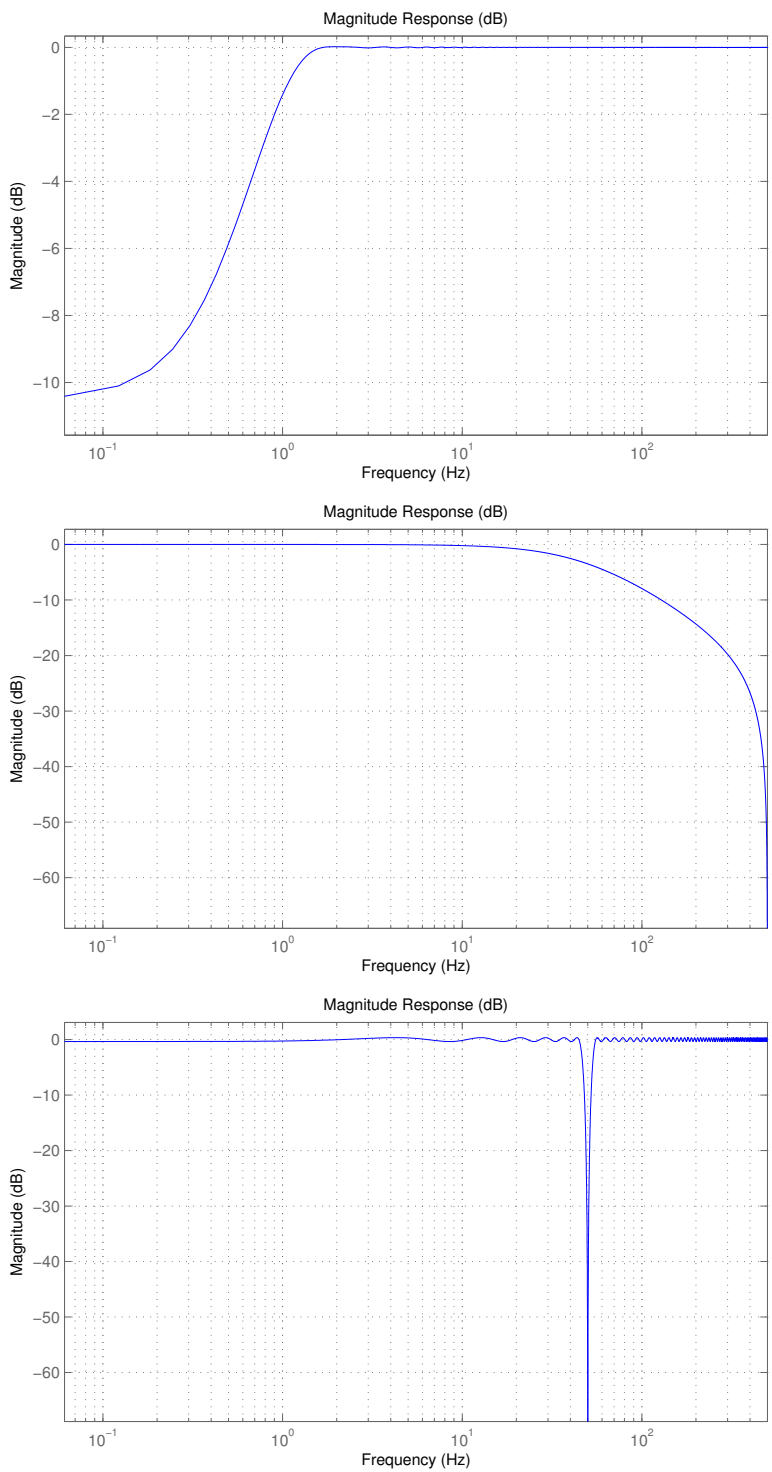

Figure 36: Magnitude response plots of the filters designed to remove ECG artifacts. On top, the high pass filter to remove baseline artifacts. In the middle a low pass filter to remove high frequency noise. On bottom, a band cut filter designed to remove power line interference at $50 \mathrm{~Hz}$. 
detected beats the user can adjust the threshold used by default by Arrhythlab, to improve the beat detection.

Once the ECG is segmented and the detected beats are supervised, the user can label each beat using the tags included in Table 6 or the events included in Table 7. In Arrhythlab, the labeling process could be done using a one-byone strategy or labeling a set of consecutive beats together only specifying the interval of pulses to label.

To help the clinician to avoid unlabeled beats, a counter of remaining unlabeled beats was included in Arrhythlab.

Table 6: Beat tags used in Arrhythlab.

\begin{tabular}{|lll|}
\hline Atrial Escape & Ventricular & Noise/Artifact \\
Junctional Escape & Ventricular R-on-T & Normal \\
Supraventricular Escape & Fusion & Supraventricular \\
Ventricular Escape & Pacemaker & unclassificable \\
Aberrated atrial & Pseudofusion & \\
\hline
\end{tabular}

Table 7: Event tags used in Arrhythlab.

\begin{tabular}{|lll|}
\hline Sinus arr. & Regular rhythm tach. & Polymorphic vent. tach. \\
Respiratory arr. & Regular vent. rhythm tach. & Vent. flutter \\
Atrial escape rhythm & Potential asystole & Vent. fibrilation \\
Alternating Pacemaker & Bigeminism & Sinus brandi. \\
Respiratory changes P. & Trigeminism & Junctional brandi. \\
Atrial .abs. FF. arrh. & Sinus tach. & Atrial ectopic brandi. \\
Atrial escape rhythm & Junctional reentry tach. an. & AV Block II mbtz I \\
AV Block I & Junctional reentry tach. & AV Block II mbtz II \\
Atrial bigemisism & Atrial chaotic tach. & AV Block III dis AV \\
Atrial interm. bigeminism & Atrial ectopic tach. & Non spec. supravent. tach. \\
Vent. regular rhythm & AV junctional tach. & Non spec. tach. \\
Escape rhythm & Monomorphic vent. tach & \\
\hline
\end{tabular}

\subsubsection{The graphical user interface}

The main window of Arrhythlab is divided in the following regions:

- ECG plot: In the ECG plot, the 12 leads are plotted to present a general view of the ECG record. 
- Selected beat plot: In this region a plot of the selected beat is shown to facilitate the beat examination and labeling.

- Navigation, threshold and edition region: The main buttons for navigation, threshold modification, and beat detected edition are placed in this part of the main window.

- Labeling region: In the labeling region the beat and event labels are presented with associated check boxes to label the selected beat.

An screenshot of the main menu of Arrhythlab is shown on Figure 37

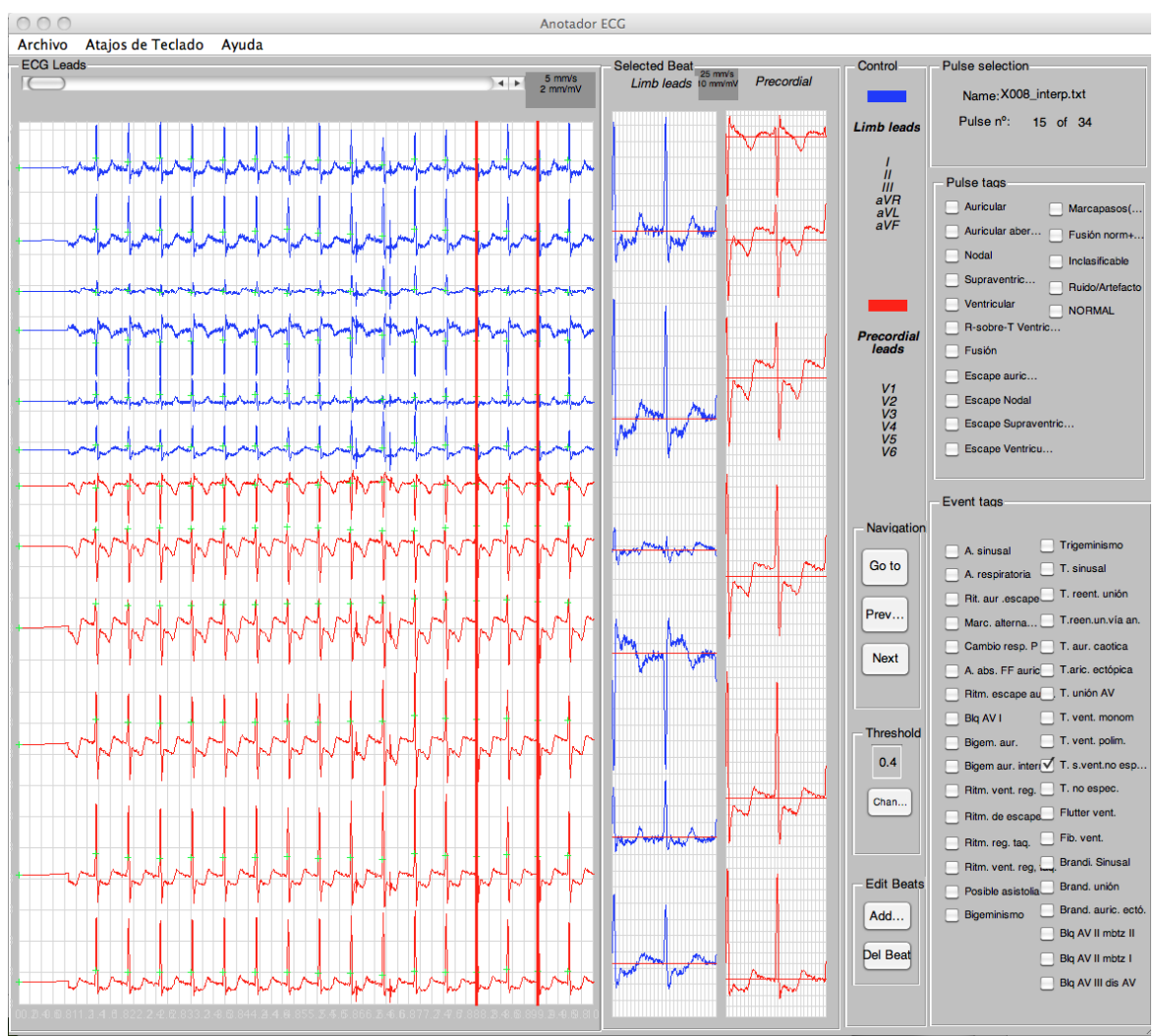

Figure 37: Screenshot of Arrhythlab main window

\subsubsection{Application}

Arrhythlab is designed to label ECG pulses for their use in arrhythmia supervised classification problems. Arrhythlab allows not only the beat labeling but also the automatic pre-processing, $\mathrm{R}$ wave detection, and beat segmentation. Arrhythlab facilitates the compilation of labeled ECG arrhythmia datasets and the export of the results to MATLAB for their analysis.

Arrhythlab is being used in the Sistema Experto en Electrocardiografia project (TSI-020302-2010-136). It has been used up to now to label ECG 
exams of 109 patients provided by two clinical centers: Hospital de la Santa Creu i Sant Pau and Hospital Universitari Germans Trias i Pujol. 

COMPATIBILITY BETWEEN $3 \mathrm{~T}^{1} \mathrm{H}$ SV-MRS DATA AND

AUTOMATIC BRAIN TUMOUR DIAGNOSIS SUPPORT SYSTEMS BASED ON 1.5 T ${ }^{1} \mathrm{H}$ SV-MRS

The assessment of biomedical signals compatibilities in the context of automatic classification problems, allows us to expand the number of signals available for the creation of classification models as well as, to detect differences between biomedical signals obtained with different technologies.

The study included in this chapter, demonstrates that 3T SV-MRS data can be used with the currently available automatic brain tumour diagnostic classifiers which were trained on databases of $1.5 \mathrm{~T}$ spectra. This will allow the existing large databases of $1.5 \mathrm{~T}$ MRS data to be used for diagnostic classification of $3 \mathrm{~T}$ spectra, and perhaps also the combination of $1.5 \mathrm{~T}$ and $3 \mathrm{~T}$ databases. Brain tumour classifiers trained with $1541.5 \mathrm{~T}$ spectra to discriminate among high grade malignant tumours and common grade II glial tumours were evaluated with a subsequently-acquired set of $1551.5 \mathrm{~T}$ and $373 \mathrm{~T}$ spectra. A similarity study between spectra and main brain tumour metabolite ratios for both field strengths ( $1.5 \mathrm{~T}$ and $3 \mathrm{~T}$ ) was also performed. Our results showed that classifiers trained with $1.5 \mathrm{~T}$ samples had similar accuracy for both test datasets $(0.87 \pm 0.03$ for $1.5 \mathrm{~T}$ and $0.88 \pm 0.03$ for $3.0 \mathrm{~T})$. Moreover, no significant differences were observed with most metabolite ratios and spectral patterns. These results encourage the use of existing classifiers based on $1.5 \mathrm{~T}$ datasets for diagnosis with $3 \mathrm{~T}{ }^{1} \mathrm{H}$ SV-MRS. The large $1.5 \mathrm{~T}$ databases compiled throughout many years and the prediction models based on $1.5 \mathrm{~T}$ acquisitions can therefore continue to be used with data from the new $3 \mathrm{~T}$ instruments.

The results presented in this chapter are published as journal paper in $\left[\mathrm{FGNV}^{+} 11\right]$.

\subsection{INTRODUCTION}

The current development of automatic brain tumour classifiers based on ${ }^{1} \mathrm{H}$ SV-MRS data has provided promising results for brain tumour diagnostic support [GGLJS ${ }^{+}$09, $\mathrm{LDS}^{+}$04, $\mathrm{DLS}^{+}$04, $\mathrm{TMM}^{+}$03]. A growing number of studies and applications have been presented in the last few years showing the ability of MRS to distinguish among different brain tumour tissue types $\left[\mathrm{TUA}^{+}\right.$06, $\mathrm{CMMB}^{+}$06, $\mathrm{LMBCV08}, \mathrm{MBCMB}^{+}$02, $\mathrm{OPB}^{+}$09, $\mathrm{SNE}^{+} 03$, $\left.\mathrm{HRF}^{+} 08, \mathrm{SJK}^{+} 10, \mathrm{VRGN}^{+} 09, \mathrm{VRJS}^{+} 12\right]$. These systems are mostly based on the pattern recognition approach, where classification models have been 
inferred from experimental data, after the extraction of relevant features [TK06, Bis95, DHS00, GNBMnR ${ }^{+}$10, CBV11, AVR12].

The learning procedures commonly used in pattern recognition assume that samples are independent and identically distributed; therefore, these classifiers are expected to be useful when classifying spectra acquired in configurations similar to those in the training data. This assumption represents a challenge when new spectra are acquired with an evolving technology, such as changing from 1.5T to 3T MR scanners. 3T scanners are becoming widely available in the clinical environment, complementing the more common 1.5T scanners. Their increased magnetic field improves SNR, and spectral resolution: the latter is particularly important for short TE spectra $\left[\mathrm{KCN}^{+} 06, \mathrm{BHB} 01\right]$ as fine structure in the $\mathrm{Gln} / \mathrm{Glu}$ region of the spectrum downfield from NAA is better resolved, and resonances downfield from water are better visualized [BHB01]. This better resolution of overlapping signals from coupled spin systems also improves metabolic characterization, thus enhancing the diagnostic abilities of MRS

Despite these advantages, it would take many years to develop databases of $3 \mathrm{~T}$ brain tumour spectra comparable to those currently available at $1.5 \mathrm{~T}$, so there is a strong incentive to use 1.5T-based classifiers to characterize $3 \mathrm{~T}$ spectra. However, the currently available $1.5 \mathrm{~T}$ based classifiers have not been validated on 3T data and it is not yet known whether we can expect a decrease in their level of performance due to differences in the overall spectral patterns. Such differences may arise from a variety of factors, both biophysical and instrumental: differences due to coupling or T2 relaxation times; and artefacts arising from water residuals or chemical shift displacement across the localization voxel. The study of Barker et al. [BHB01] showed subtle differences between the spectra obtained at the two field strengths, including better-resolved peaks of the NAA amide and glutamate/glutamine region at 3T compared to $1.5 \mathrm{~T}$ and a peak at $3.3 \mathrm{ppm}$ clearly observable in some subjects at $1.5 \mathrm{~T}$, which was less prominent at $3 \mathrm{~T}$.

Some authors have suggested the application of established 1.5T metabolite ratios for the evaluation of brain tumours at $3 \mathrm{~T}\left[\mathrm{KCN}^{+} 06\right]$. Additionally, Roser et al. [RHM ${ }^{+}$97] concluded that the change from $2 \mathrm{~T}$ to $1.5 \mathrm{~T}$ had no measurable deleterious effect on multidimensional metabolic classification for assignment of glial brain tumours.

To analyze this behavior, we have performed a study of the performance of $1.5 \mathrm{~T}$ based classifiers when tested with $3 \mathrm{~T}$ spectra. The main goal of our study was to test the compatibility of 1.5 and $3 \mathrm{~T}$ data when they are used on automatic brain tumour CDSS [BE07, $\left.\mathrm{SGG}^{+} 01\right]$. To achieve that goal, a standard spectrum-processing protocol has been applied, and the performance of different feature extraction methods and classification algorithms has been analyzed. Classifiers already included in a CDSS [GVMJS ${ }^{+} 07, \mathrm{SGGV}^{+} 08$ ] have been tested on new 1.5T and 3T acquisitions, and their performance has been evaluated on the well-known test problem $\left[\mathrm{GGTV}^{+} 08\right]$ to discriminate High Grade Malignant (HGM) - glioblastoma and metastases - tumours, 
from Common Grade II Glial- astrocytomas grade II, oligoastrocytoma and oligodendroglioma - $(\mathrm{CG} 2 \mathrm{G})$ tumours. A hypothesis testing of the accuracy of each combination of feature extraction and classification methods was performed to evaluate the compatibility between $3 \mathrm{~T}$ data and the existing classifiers based on 1.5T data. Finally, a study on the differences between spectra obtained at both field strengths was performed, analyzing the differences between the main brain metabolite ratios and spectra shapes.

\subsection{MATERIALS AND METHODS}

\subsubsection{In vivo ${ }^{1} H$ SV-MRS datasets}

Three datasets were used in our study. The first one is a training $1.5 \mathrm{~T}{ }^{1} \mathrm{H}$ SV-MRS dataset accrued during the INTERPRET EU project [TUA $\left.{ }^{+} 06\right]$. The second dataset is part of the 1.5T dataset compiled in the eTUMOUR EU project [The07], and was used as an independent test set to evaluate the performance of the classifiers. The third dataset is a new 3T dataset used to evaluate the performance of the $1.5 \mathrm{~T}$ classifiers on $3 \mathrm{~T}$ cases. All the datasets were obtained using MR scanners of three major manufacturers (Philips, General Electric and Siemens) in ten international centers. The number of cases used in each dataset is shown in Table 8.

The 1.5T training dataset included 154 1.5T short TE SV-MRS spectra. The acquisition protocols included PRESS or STEAM sequences with the following spectral parameters: TR of $1600-2020 \mathrm{~ms}$, TE of 20 or $30-32 \mathrm{~ms}$, spectral width of $1000-2500 \mathrm{~Hz}, 512,1024$, or 2048 data-points, as described in previous studies $\left[\mathrm{TMM}^{+} 03\right]$. All these cases were validated using a standard quality control protocol carried out by the INTERPRET Clinical Data Validation Committee and expert spectroscopists [TUA ${ }^{+} 06, \mathrm{VdGJSH}^{+} 08$ ], and all had a histopathological diagnosis.

The 1.5T test dataset included 155 1.5T short TE SV-MRS spectra. These spectra were validated by an expert spectroscopist panel, and the histopathological diagnosis of these cases was also available. The acquisition protocols included PRESS and STEAM with spectral parameters: TR of $1500-2000 \mathrm{~ms}$, TE of $30-31 \mathrm{~ms}$, spectral width of $500-2500 \mathrm{~Hz}, 512,1024$, or 2048 data-points.

The 3T test dataset included 37 spectra and came from two different sources. The first 21 spectra were obtained in the eTUMOUR project, including 4 CG2G tumours and 17 HGM tumours. Their histopathological diagnoses were also available. The scanner used was GE Signa 3T. The acquisition parameters included PRESS sequences with spectral parameters: TR of 2000 - 5000ms, TE of $30 \mathrm{~ms}$, spectral width of $1000 \mathrm{~Hz}, 2048$ data-points. The remaining 16 spectra were acquired at the Hospital Quirón of Valencia on a Philips scanner. There were 11 histopathology proven HGM tumours and 5 CG2G tumours in which the diagnosis was made on clinical grounds, radiological appearance, and follow up. The acquisition protocols included 
Table 8: Number of $1 \mathrm{H}$ SV-MRS spectra in each dataset per tumour type. High Grade Malignant (HGM), consisting of glioblastomas and metastases, and common grade II glial (CG2G) consisting of astrocytomas grade II, oligodendrogliomas and oligoastrocytomas.

\begin{tabular}{cccc}
\hline Dataset & CG2G & HGM & Total \\
\hline 1.5T Training & 34 & 120 & 154 \\
1.5T Test & 53 & 102 & 155 \\
3T Test & 9 & 28 & 37 \\
\hline
\end{tabular}

a PRESS sequence with spectral parameters: TR of $1800-2000 \mathrm{~ms}$, TE of 32 ms, spectral width of $2000 \mathrm{~Hz}, 1024$ data-points. The TE's were optimized for a satisfactory SNR without losing any metabolite resonances or showing coupling variations.

In Figure 38, spectra sample of the two tumour types included in the study are presented from 1.5T and 3T MR scanners.

\subsubsection{MRS processing}

A common MRS processing pipeline, previously used in the INTERPRET and eTUMOUR EC projects, and included in the CDSS software they developed $\left[\mathrm{SGGV}^{+}\right.$08, PROCJS ${ }^{+}$08, The07], was applied in the present study. Each spectrum was semi-automatically pre-processed using a pipeline consisting of 1) eddy current correction applied to the water-suppressed free induction decay of each case using the Klose algorithm [Klo90]; 2) zero and first order manual phase correction; 3) residual water resonance suppression by the Hankel-Lanczos singular value decomposition time-domain selective filtering using 10 singular values and a water region of [4.33, 5.07] ppm; 4) an apodization with a Lorentzian function of $1 \mathrm{~Hz}$ of damping; 5) zero filling, to increase the number of points of the low resolution spectra to the maximum number used in the acquisition protocols (2048); 6) baseline offset subtraction, estimated as the mean value of the regions [11,9] and [-2,-1] ppm; 7) normalization of spectra to the Euclidean norm using the regions [2.7, 4.33] [5.07, 7.1] ppm; 8) additional frequency alignment check of the spectrum by referencing the ppm-axis to the total creatine at $3.03 \mathrm{ppm}$ or to the choline-containing compounds at $3.21 \mathrm{ppm}$ or the mobile lipids at 1.29 ppm, depending on the SNR and the tumor pattern; and finally 9) reduction of the number of points of the spectra, using 512 points for the defined region of $[-2.7,7.1] \mathrm{ppm}$. No corrections for $\mathrm{T} 1$ or $\mathrm{T} 2$ relaxation effects were made to the spectra prior to the pattern recognition analysis.

The software used to pre-process the spectra was jMRUI $3.0\left[\mathrm{VVV}^{+} 96\right]$ in batch mode (steps 1-5) and jDMS [PROCJS ${ }^{+} 08$ ] (steps 6-9). 

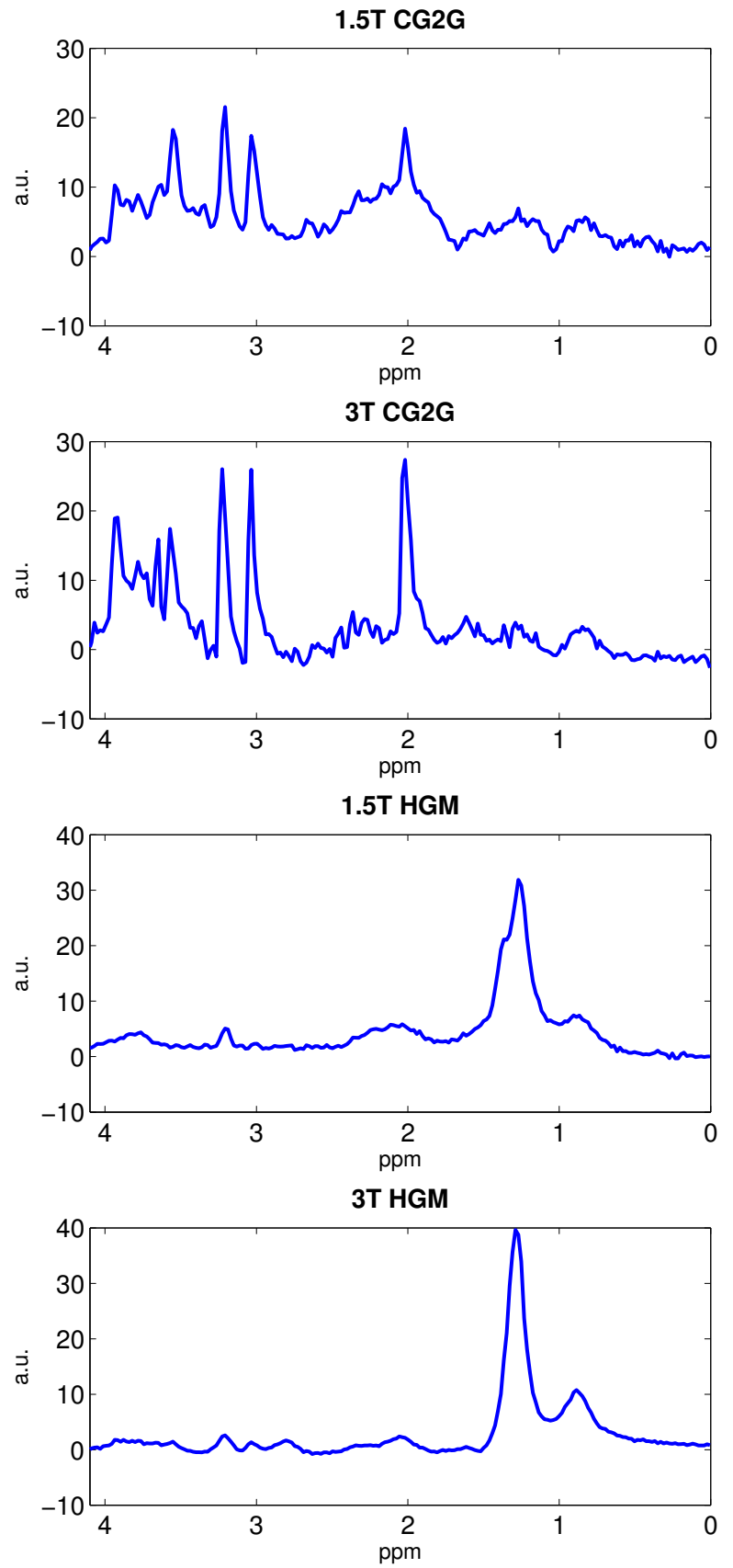

Figure 38: Example STE spectra from 1.5 and 3T MR scanners of the two tumour types used in this study. 


\subsubsection{Feature extraction method}

One of the major problems in spectral classification arises from the number of variables that represent the full region of interest (190 data points in this case). The use of a large number of variables in classification problems generally overfits the training sample and generalizes poorly to new samples. To overcome this problem, the variables are usually transformed into a reduced representation set of features that maintains the most representative information $\left[\mathrm{LPGG}^{+} 08\right]$.

Two different FE methods were used in this study: SW and Peak Integration (PI). SW is a suboptimal greedy hill climbing approach [RN95]. This algorithm was applied with the Mahalanobis squared distance, for selecting relevant subsets of features based on the performance measure of the training classification. PI computes the value of the area under the peaks of the most relevant metabolites (see Table 13) as a representation of the significant information contained in the spectra. To obtain the areas under the peaks we considered an interval of $0.15 \mathrm{ppm}$ from the assumed peak centre (Figure 39). The metabolites used were mobile lipids, Lac, Ala, NAA, acCr, total Cho, glutamine, glutamate, $\mathrm{mI}+$ glycine, and Tau.

These two methods were selected to include in the study an example of a FE method (e.g. PI) and an example of a FS method (e.g.SW). Moreover, they were selected in order to include an example of FE that does not assume prior knowledge (e.g.SW) and also an example of a FE (in the general sense) that uses prior knowledge based on clinicians expertise (e.g. PI).

\subsubsection{Classifiers}

Fisher's LDA, KNN and ANN were used for the classification. All of them have been successfully applied in a CDSS for brain tumor diagnosis based on MRS [GGLJS $\left.{ }^{+} 09, \mathrm{TUA}^{+} 06, \mathrm{OLB}^{+} 07\right]$. A brief description of these classification methods can be seen on Section 2.5.2.

\subsubsection{Performance Measures}

To determine the performance of a classifier, the Accuracy (ACC) and Geometric Mean of Recalls (G) evaluation metrics were selected (see section 2.5.4 for a detailed description). The G nonlinear metric is especially useful to avoid overestimating the classifier performance when predictions show a high variance over the classes. 


\subsubsection{Statistical Analysis}

To evaluate the compatibility between $3 \mathrm{~T}$ data and the classifiers based on 1.5T data, the Pearson's $\chi^{2}$ test $(\alpha=0.05)$ for a contrast hypothesis was performed on the accuracy for each combination of feature extraction and classification methods.

To complete the compatibility study between 1.5 and 3T SV-MRS, the differences between the main brain metabolite ratios were compared for both field strengths. Hence, non-parametric Mann-Witney $U$ test and boxand-whisker diagrams were calculated. The peak area ratios included in this study were: $\mathrm{mI} / \mathrm{Cr}$, where $\mathrm{mI}$ at short may also include signals from Gly which overlaps the $\mathrm{mI}$ peak), Cho/ Cr, Cho/ NAA, and (Lipids+ Lac)/Cr.

The software used to perform the statistical analysis was MATLAB 2008.

\subsection{RESULTS}

Every MR spectrum was processed using the afore-mentioned pipeline, and the feature extraction methods were applied to the 1.5T training dataset. The significant points selected by the $\mathrm{SW}$ algorithm from the spectra region of interest ([4.1-0.5] ppm) were 3.97, 3.76, 3.57, 3.30, 3.11, 3.03, 2.34, 1.25, 0.98, $0.85 \mathrm{ppm}$ (Figure 39).

Before dealing with performances of the classifiers, an analysis of MRS patterns at $1.5 \mathrm{~T}$ and $3 \mathrm{~T}$ was performed. A qualitative comparison between the mean spectra of each class (HGM, CG2G) for the three datasets (1.5T train, 1.5T test, 3T test) showed that the mean spectra tend to fall inside the region of coincidence among the three patterns (Figure 39). Also an analysis of the differences in metabolite ratios has been done performing the Mann-Witney U nonparametric test and using box-and-whisker diagrams (see Figure 40). In all cases, the p-values obtained were greater than 0.05 , which indicates no significant difference among the datasets.

The performance of the classifiers on the 1.5T training dataset was estimated by a 10-fold Cross Validation (CV). The results are presented in the first row of Table 9.

In order to evaluate the 1.5T-based classifiers, the $1.5 \mathrm{~T}$ and $3 \mathrm{~T}$ test datasets were used as independent tests. Their performance values are presented in the second and third rows of Table 9. In both cases, the classifiers based on $\mathrm{KNN}+\mathrm{PI}$ and ANN+PI gave better performance in terms of G and ACC; however these differences were non-significant. The results obtained showed a p-value greater than 0.1 for every hypothesis contrast. 

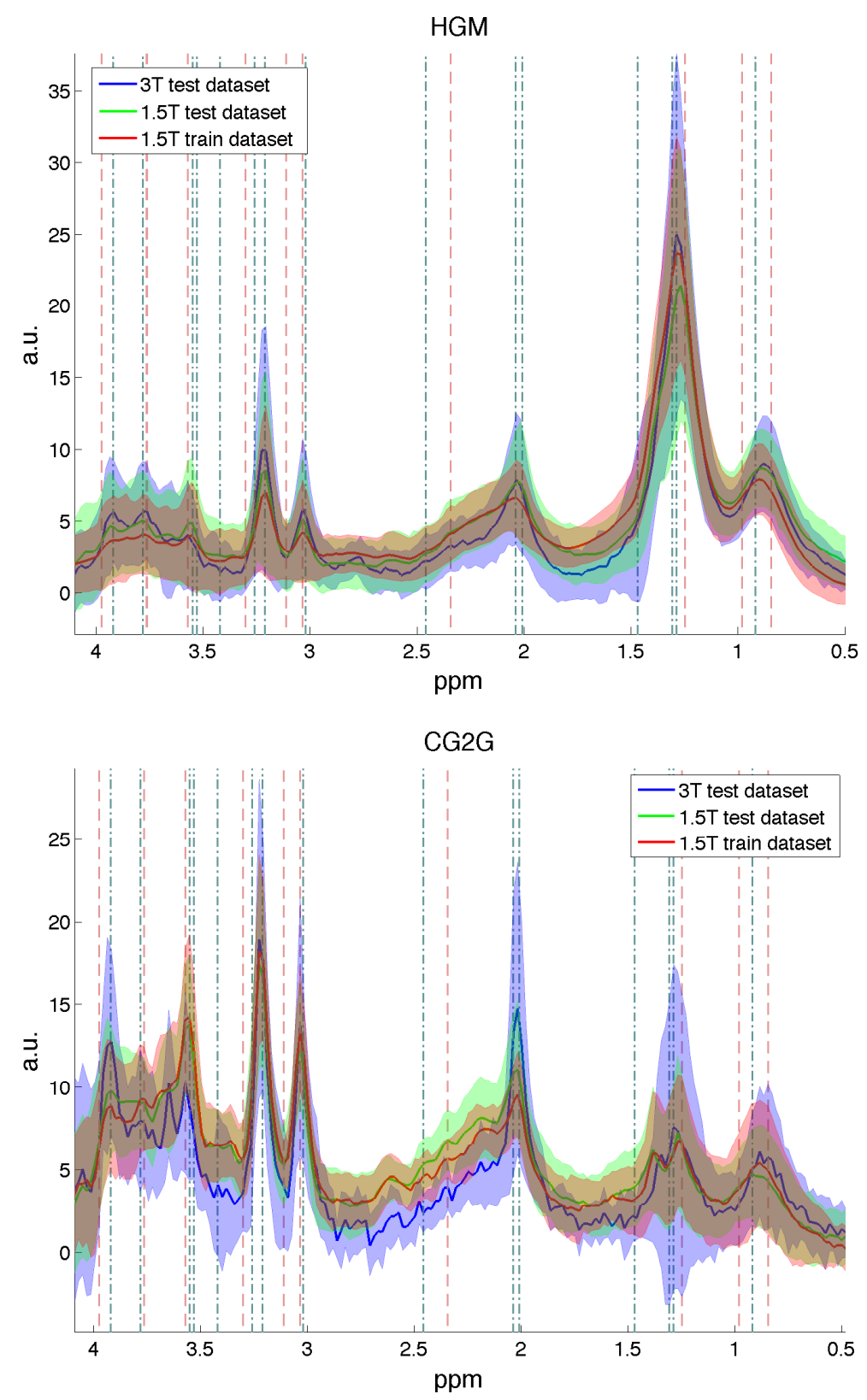

Figure 39: Spectral patterns of each of the classes (HGM, CG2G) for the 1.5T training, $1.5 \mathrm{~T}$ test and $3 \mathrm{~T}$ test data sets. The mean spectrum of each dataset is shown as a solid line and its standard deviation in shadowed colour. The darker brown region corresponds to the region of coincidence among the three patterns. The points selected by the stepwise algorithm are shown as vertical red dashed lines. The points selected as centroids for the peak integration method are shown as vertical green dash-dot lines. 

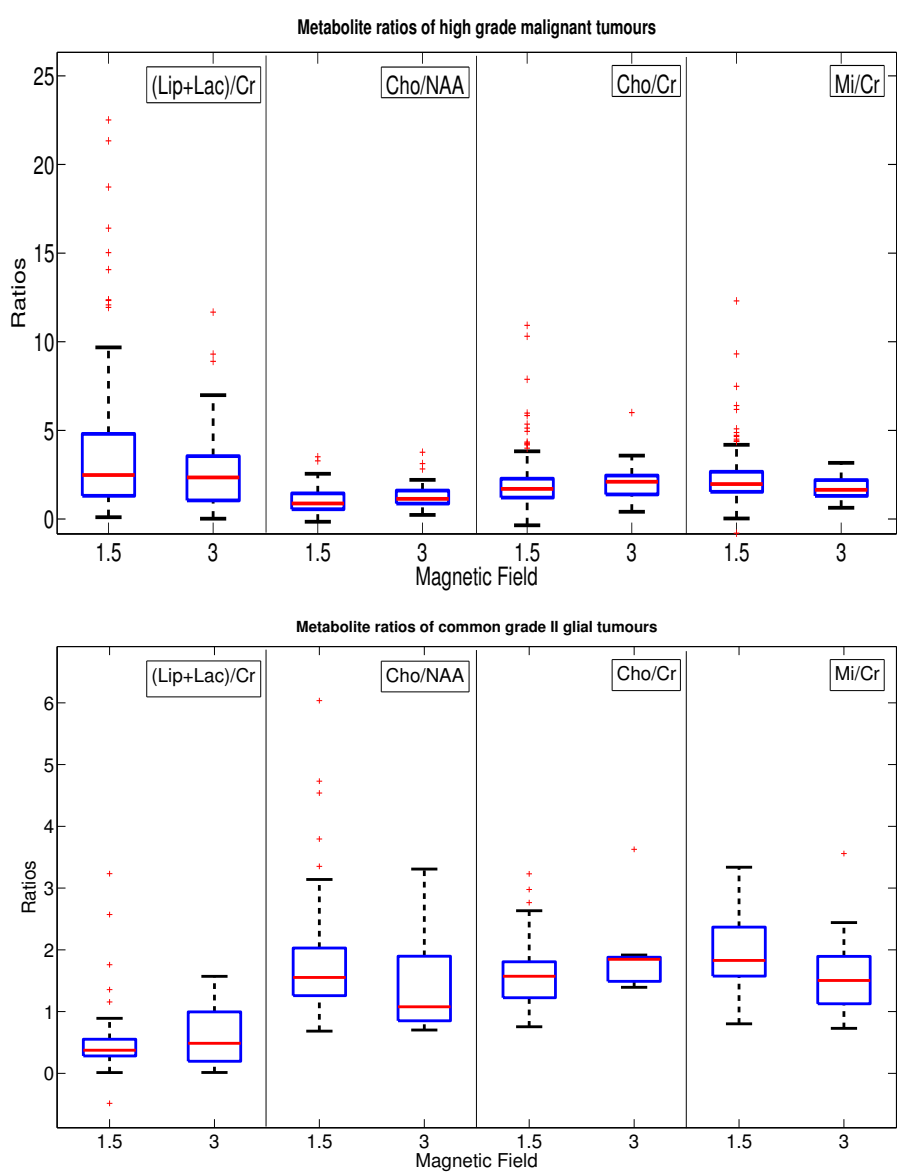

Figure 40: Box-and-whisker diagrams for each tumour class (HGM,CG2G), comparing the main brain metabolite ratios obtained for both field strengths. Outliers are shown as red crosses.

Table 9: Classification results obtained for the three datasets. In the columns, the results for each combination of feature extraction method (SW and PI) and classifier (KNN, LDA and ANN) expressed in terms of accuracy (ACC) and geometric mean of success $(\mathrm{G})$. CV has been applied in the case of the 1.5T training dataset in order to obtain the $\mathrm{G}$ and the ACC estimators.

\begin{tabular}{l|ccccccccccccc}
\hline \multirow{2}{*}{ Dataset } & \multicolumn{2}{|c}{ KNN+SW } & \multicolumn{2}{c}{ KNN+PI } & \multicolumn{2}{c}{ LDA+SW } & \multicolumn{2}{c}{ LDA+PI } & \multicolumn{2}{c}{ ANN+SW } & \multicolumn{2}{c}{ ANN+PI } \\
& ACC & G & ACC & G & ACC & G & ACC & G & ACC & G & ACC & G \\
\hline 1.5T train $(\mathrm{CV})$ & 0.93 & 0.90 & 0.91 & 0.88 & 0.93 & 0.91 & 0.91 & 0.90 & 0.95 & 0.94 & 0.93 & 0.90 \\
\hline 1.5T test & 0.87 & 0.87 & 0.90 & 0.90 & 0.84 & 0.83 & 0.82 & 0.79 & 0.88 & 0.88 & 0.91 & 0.92 \\
\hline 3T test & 0.89 & 0.89 & 0.91 & 0.90 & 0.84 & 0.82 & 0.84 & 0.82 & 0.91 & 0.90 & 0.89 & 0.89 \\
\hline
\end{tabular}




\subsection{DISCUSSION}

We have tested the compatibility between the two currently coexisting clinical MR scanners of 1.5T and 3T, both for the development of new classifiers for tumour diagnosis support and also for the use of the existing ones based on 1.5T spectra. Although our present study was focused on two tumour classes ( $\mathrm{HGM}, \mathrm{CG} 2 \mathrm{G}$ ), the results of $\mathrm{Kim}$ et al. $\left[\mathrm{KCN}^{+} 06\right]$ also suggest that we may apply the established methods concerning the metabolite ratios obtained from 1.5T spectra for the evaluation of brain tumours at 3T. Thus it may be possible to extend the results to other focal brain lesions as long as their classification is not heavily dependent on features relating to metabolites that are well resolved in 3T MRS but not in 1.5T (e.g. Glu and Gln). [BHB01].

This initial study was designed to provide evidence of whether we will be able to further benefit from the effort made throughout many years in the compilation of large databases of $1.5 \mathrm{~T}$ MRS data by using them to analyze results obtained at $3 \mathrm{~T}\left[\mathrm{The} 07, \mathrm{TUA}^{+} 06\right]$.

The classification algorithms (ANN, LDA, KNN) as well as the feature extraction methods (PI, SW) used in this study have been selected from those used in published works to facilitate validation of the results [GGLJS ${ }^{+} 09$ ]. Also, the algorithms already implemented in diagnosis support systems $\left[\mathrm{SGGV}^{+} 08\right.$, PROCJS $\left.{ }^{+} 08\right]$ have been taken into account to increase the relevance of the conclusions obtained.

The training performances obtained show consistent results. Firstly, most of the points automatically selected by the SW algorithm are related to or are close to the metabolite peaks chosen by the spectroscopists: $\mathrm{Cr}$ (3.03 and 3.93 ppm), Ala (3.77 ppm), mI (3.53 and 3.26), Cho (3.21 ppm), Cr (3.02 ppm), Lac (1.30 ppm), Tau (3.30ppm), Gly (3.56ppm), NAA (2.02ppm), glutamine (2.14 $\mathrm{ppm})$, glutamate (2.35ppm) and lipids (0.92 and 1.29ppm). Secondly, the performances obtained on the training data using a cross validation method (first row of Table 9) are comparable with the ones reported by García-Gómez et al, in [GGLJS ${ }^{+} 09, \mathrm{GGTV}^{+}$08].

Since the cross validation method is optimistic compared to an independent test, a performance reduction occurs when using the trained classifiers on the test samples. This expected performance reduction can be observed between the first and second row of Table 9.

Because no significant difference on ACC was achieved between the two test datasets, we consider that the performances of the 1.5T based classifiers when tested on either 1.5T test set or 3T test set are comparable for the tumour classes analyzed. These results establish the possibility of using existing 1.5T based classifiers on 3T SV-MRS spectra. This agrees with the results obtained for $1.5 \mathrm{~T}$ and $2 \mathrm{~T}$ by Roser et al. in $\left[\mathrm{RHM}^{+}\right.$97] when using multidimensional metabolic classification for assignment of glial brain tumors.

This conclusion was reinforced by the similarities observed between both spectra types (1.5T and 3T). For each tumour type obtained for 1.5T and 3T 
the spectral patterns fell inside the coincidence region defined by the spectra standard deviations, and the height of the metabolite peaks were similar (see Figure 39). Nevertheless, there were clear differences in the mean 3T spectra compared to the $1.5 \mathrm{~T}$ spectra in the region of 3.5 to $4 \mathrm{ppm}$ where strong signals from coupled spins can be found.

Furthermore, a comparison between the main metabolite ratios at the two magnetic field strengths was performed. The Mann-Witney U test showed no significant differences between metabolite ratios at the two magnetic fields except for the case of the Myo-Inositol / Creatine ratio in HGM tumours. The box-and-whisker diagrams (see Figure 40) showed that in all cases the intervals defined by the first and third quartile contained common values for both magnetic fields. This agrees with the conclusions obtained by Kim et al. in $\left[\mathrm{KCN}^{+} 06\right]$.

Although this study focuses on the compatibility between 3T MRS data and automatic brain tumour diagnosis support systems based on 1.5T MRS, we found interesting for this discussion to compare the classification performance when using both $1.5 \mathrm{~T}$ and $3 \mathrm{~T}$ MRS samples to train and test the classification models and also when using only 1.5T samples. To do so, the classifiers included in this study were trained and tested using a mixed dataset (309 1.5T +37 3T) and also using a unmixed dataset that only contains 1.5T MRS samples (309 1.5T MRS samples). A bootstrap evaluation strategy [JDC87] (400 blocks) was used to obtain the performance results. The results show no significant differences between the performances obtained when using the mixed dataset $(A C C=0.88 \pm 0.01)$ and the unmixed dataset $($ ACC $=0.88 \pm 0.01)$.

In future work, incremental learning algorithms [GC00] will be introduced to generate new classifiers based on 1.5T data that could learn from new 3T cases. These techniques will increase the performance of the classifiers over the course of time, and will provide more reliable results. Also the generalization of this study to the case of multi-voxel MRSI data is an important goal for future work. This is not a trivial problem, because of the substantial differences between the two data types, both in the acquisition and processing of the spectra. Moreover the differences between 1.5T and 3T datasets maybe larger in the case of MRSI data $\left[\mathrm{GGL}^{+} 01\right]$ even with long TE if the advantages of 3T are used for rapid MRSI data acquisitions [DMLB06]. 

One of the current challenges in automatic brain tumor classification based on MRS is the improvement of robustness of the classification models that explicitly account for the probable breach of the independent and identically distributed conditions in the MRS data points. To contribute to this purpose, a new algorithm for extracting discriminant MRS features of brain tumors based on a functional approach is presented. RSFDA is based on the functional data analysis formalism using non-uniformly distributed B-splines according to spectra regions that are highly correlated. An exhaustive characterization of the method is presented in this chapter using controlled and real scenarios. The performance of RSFDA has been compared with other widely used feature extraction methods. In all simulated conditions, RSFDA has proven to be stable with respect to the number of variables selected and with respect to the classification performance against noise and baseline artifacts. Furthermore, with real multi-center datasets classification, RSFDA obtains better performance than the feature extraction methods used for comparison. Another advantages of the method proposed are its usefulness in selecting the optimal number of features for classification and its simplified functional representation of the spectra, which contributes to the interpretability of the results.

The RSFDA algorithm was submitted for publication to NMR in Biomedicine journal.

\subsection{INTRODUCTION}

In recent years, numerous advances have taken place in the automatic analysis of MRS for the diagnosis of brain tumors. CDSSs have incorporated machine learning techniques to extract metabolic information from MR spectra for the automatic classification of tumor tissue. $\left[\mathrm{SGGV}^{+}\right.$09, $\mathrm{TUA}^{+}$06, $\mathrm{PRJSM}^{+}$10].

One critical step in the development of robust classifiers is the selection of the optimal features that synthesize the most discriminant information of MRS spectra in a reduced set of independent variables. To make this selection, FE methods need to take into account the probable breach of the i.i.d. condition in the analyzed MRS data points. Moreover, for classification purposes, the extracted features need to retain the most discriminant information instead of the variability information. 
Typically, methods based on MRS quantification or based on machine learning are used to obtain these features. Quantification methods are useful in providing valuable information for biological interpretation of spectral data. Despite this, quantification methods such as AQSES [PSS ${ }^{+}$07] or QUEST $\left[\mathrm{RSC}^{+} 05\right]$ are difficult to automate, are not as successful as the FE methods based on pattern recognition, and require expert knowledge $\left[\mathrm{LPGG}^{+} 08, \mathrm{MLB}^{+} 06\right]$. There are, however, other quantification algorithms included in softwares such as LCModel [Pro01] or TARQUIN [WRK $\left.{ }^{+} 11\right]$ that are automatic, although their classification accuracy has not been proven better than the classical methods of FE such as PCA, ICA, or PI $\left[\mathrm{SMdE}^{+}\right.$05, $\mathrm{LPGG}^{+}$08, RFGOH12]. FE methods based on machine learning strategies have shown more favorable results in their application to MRS in brain tumor cases $\left[\mathrm{LPGG}^{+} 08, \mathrm{OLB}^{+}\right.$07]. They are also easier to automate and, therefore, easier to incorporate in a DSS for clinical use $\left[\mathrm{LPGG}^{+} 08\right.$, $\mathrm{MLB}^{+}$06, $\mathrm{FGNV}^{+} 11, \mathrm{GGLJS}^{+} 09, \mathrm{OmOV}^{+}$10].

Despite these advantages, most of the FE methods that are widely used in these studies are not optimized for the classification of continuous signals. On the one hand, some of them are not optimized for classification problems in the sense that they are optimized to retain the maximum information contained in the dataset using the minimum number of features. However, this information does not have to necessarily coincide with the most useful one for discriminating. This is the case for the ICA or PCA algorithms. On the other hand, FE methods, such as, the FC, SW, or ReliefF have some disadvantages when they are used on continuous signals. They are based on a point-to-point approach, do not take into account the implicit correlation between nearby points in the signal, and are therefore susceptible to include highly correlated redundant variables.

In order to overcome these drawbacks, this work introduces a new feature extraction method - RSFDA - for the classification of brain tumors based on MRS. The RSFDA segments the signal according to discriminant regions that are composed of highly correlated points. A functional description based on non-uniformly distributed B-splines is used to model the spectra according to the highly correlated regions obtained. The knots for this functional description are defined as the edges of the obtained regions. By doing so, the RSFDA ensures an intra region detail using a low number of variables. Hence, a new parametric space of the MRS signals is generated based in the set of coefficients of each B-spline. The RSFDA provides easily interpretable results, does not require prior knowledge (such as quantification or PI), and is fully automatic.

The proposed FE method has beed evaluated by means of simulated and real multicenter data in different binary classification problems. A comparison with the performance of other FE methods such as FC and PCA has also been done. Finally, LDA and the KNN classification methods have been used to evaluate the classification models with the new parametric space. 


\subsection{MATERIALS}

We designed two experiments for this work. In the first experiment, ideal MRS datasets were simulated from a FID physical model. Five spectra groups were defined to simulate five brain tumor classes. These ideal datasets were modified to create realistic ones by the introduction of typical MRS artifacts like noise, baseline distortions, or frequency shift. In the second experiment, the eTUMOUR [The07] and INTERPRET $\left[\mathrm{TMM}^{+} 03\right.$, $\mathrm{JSAM}^{+} 06$ ] datasets were used for a comparative evaluation of RSFDA with state-of-the-art methods. We have studied binary classification problems corresponding to discrimination among $\mathrm{CG} 2 \mathrm{G}$ consisting of astrocytomas grade II, oligodendrogliomas and oligoastrocytomas, GBM, MEN and MET brain tumor types. These classes were selected in accordance with the standard classification problems described in the literature $\left[\mathrm{LPGG}^{+} 08\right.$, $\mathrm{LDS}^{+}$04, $\mathrm{OLB}^{+} 07, \mathrm{GGLJS}^{+}$09, RFGOH12]. The number of available cases is described in Table 10.

Table 10: Number of ${ }^{1} \mathrm{H}$ SV-MRS real spectra in each multicenter dataset per tumor type. Common grade II glial (CG2G) consisting of astrocytomas grade II, oligodendrogliomas and oligoastrocytomas, Metastasis (MET), Meningiomas (MEN) and Glioblastomas (GBM).

\begin{tabular}{cccccc}
\hline Dataset & CG2G & GBM & MEN & MET & TOTAL \\
\hline INTERPRET & 26 & 57 & 45 & 33 & 161 \\
eTUMOUR & 105 & 207 & 58 & 74 & 444 \\
\hline TOTAL & 131 & 264 & 103 & 107 & 605 \\
\hline
\end{tabular}

\subsubsection{Simulated MRS dataset}

A simulated MRS dataset was created to study the behavior of the proposed method in classification problems with different artifacts that to a greater or lesser extent commonly affect MRS spectra. Frequency shift, baseline distortion and noise are the artifacts considered in the study. Fifteen datasets (five per artifact) consisting of 75.000 spectra were simulated to analyze the influence of each of these artifacts. An example of a real and a simulated MRS case used in this study is presented in Figure 41 


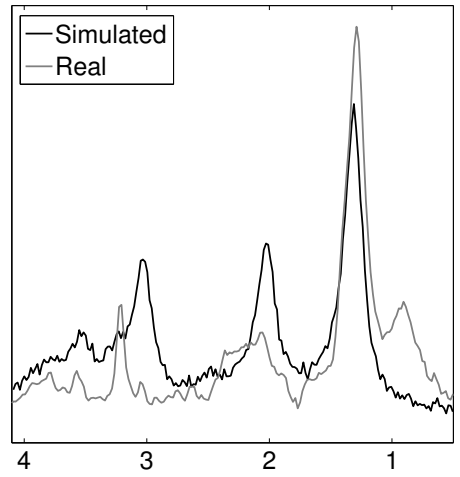

Figure 41: Example of real (gray line) and simulated (black line) MRS data. The real MRS spectrum corresponds to a GBM MRS tumour case. The simulated MRS spectrum have obtained with a $\sigma=0.05$ and without baseline and frequency shift artifacts.

\section{Ideal dataset}

An MRS Lorentzian FID physical model was used to obtain the simulations of the ideal dataset [VSVHVH01]. The time-domain signal $y(t)$ induced by a precessing magnetization and detected in the NMR scanner can be described by an exponentially decaying complex-valued sinusoidal (see Equation 58) for each component $k$.

$$
y(t)_{k}=a_{k} e^{-\frac{t}{T_{2, k}}} e^{j\left(2 \pi f_{k} t+\phi_{k}\right)},
$$

where an amplitude $a_{k}$ is proportional to the number of nuclei, a decaying constant is equivalent to the reciprocal $1 / T_{2, k}$ of the transverse relaxation time of the nucleus, a frequency $f_{k}$ is equal to the difference between the frequency of the NMR spectrometer and the resonance frequency of the nucleus, and $\phi_{\mathrm{k}}$ is the overall (zero-order) phase. In the case of multiple precessing magnetization associated to different metabolites, the model of the FID defined in Equation 58 becomes:

$$
\operatorname{FID}(t)=\sum_{k=1}^{K} y(t)_{k}=\sum_{k=1}^{K} a_{k} e^{j \phi_{k}} e^{\left(-d_{k}+j 2 \pi f_{k}\right) t},
$$

where $K$ represents the number of resonances and $j=\sqrt{-1}$. The damping $\mathrm{d}_{\mathrm{k}}=1 / \mathrm{T}_{2}$ provides information about its mobility, molecular information, and inhomogeneities in the field.

Five classes of spectra were simulated using this FID model. Each of these classes was obtained by mean metabolite concentrations defined by $\bar{a}_{c l a s s, k}$ and by the mean damping $\overline{\mathrm{d}}_{\mathrm{class,k}}$. A first assumption was made establishing 
the same damping factor for all classes $\overline{\mathrm{d}}_{\mathrm{class}, \mathrm{k}}=\overline{\mathrm{d}}=0.036$. For the metabolite concentrations, mean values of $\bar{a}_{\text {class }}$ were selected for each class with the values specified in Table 11. To generate each spectrum of each class, a value of $a=\bar{a}_{\text {class }}+\epsilon_{\text {class }}$ was used, where $\epsilon_{\text {class }}=$ $\mathcal{N}\left(\bar{a}_{\text {class }}, 0.65 \cdot \bar{a}_{\text {class }}\right)$, simulating the intraclass variability $(0.64 \pm 0.7)$ estimated from the STE-MRS cases included in eTumour and INTERPRET databases corresponding to the tumour types included in this work (MET,MEN, CG2G, GBM). Finally, a FFT was applied to all of the simulated FID signals in order to obtain the ideal spectra dataset (see Figure 43). In Figures 43 and 42 the mean and deviation of the ideal and real spectra classes included in this study are presented to allow visual comparison between the real and ideal MRS datasets used in this work.

\section{Baseline distorsion}

The first artifact introduced in the simulated dataset was the baseline distortion. This artifact is related to the corruption or omission of early time domain data points, and bad suppression of water ${ }^{1} \mathrm{H}$ signal. In this work, we have modeled the baseline distortion by means of a gaussian function as shown in 60.

$$
s_{\text {baseline }}=s_{\text {ideal }}+\zeta \cdot \max \left[s_{\text {ideal }}\right] \cdot \mathcal{N}\left(\mu, \sigma_{b}\right),
$$

where $\mathcal{N}$ is a Gaussian function determined by $\mu=\mathcal{U}(-1,1) \cdot 4.5 \mathrm{ppm}, \zeta$ controls the amount of baseline distortion introduced, and $\sigma_{b}=U(0,1)+1$ ppm. To study the influence of the baseline in the FE methods, different baseline distortion magnitudes were used, defining five values of $\zeta$ ranging from 0 to 0.7 linearly. 
Table 11: Amplitude values $\bar{a}$ of metabolites and other molecules observed in Short TE for for each simulated classes.

\begin{tabular}{|c|c|c|c|c|c|}
\hline Metabolites & $\bar{a}_{1}$ & $\overline{\mathrm{a}}_{2}$ & $\bar{a}_{3}$ & $\overline{\mathrm{a}}_{4}$ & $\overline{\mathrm{a}}_{5}$ \\
\hline L2 (0.92ppm) & 2 & 1 & 1 & 1 & 10 \\
\hline L1 (1.29ppm) & 10 & 1 & 3 & 10 & 10 \\
\hline Lac (1.31 ppm) & 2 & 1 & 2 & 10 & 10 \\
\hline $\operatorname{Ala}_{1}(1.47 \mathrm{ppm})$ & 5 & 2 & 8 & 2 & 10 \\
\hline NAc (2.01ppm) & 3 & 8 & 3 & 8 & 8 \\
\hline $\mathrm{Cr}_{1}(3.02 \mathrm{ppm})$ & 4 & 7 & 4 & 7 & 7 \\
\hline $\mathrm{Cr}_{2}(3.92 \mathrm{ppm})$ & 1 & 1.5 & 1 & 1.5 & 1.5 \\
\hline Cho (3.21ppm) & 1 & 1.2 & 1 & 1.2 & 1.2 \\
\hline Gly (3.55ppm) & 6 & 3 & 6 & 3 & 3 \\
\hline $\mathrm{Glx}_{1}(\mathrm{Glu}+\mathrm{Gln})(2.04 \mathrm{ppm})$ & 3 & 3.2 & 3 & 3.2 & 3.2 \\
\hline $\mathrm{Glx}_{2}(\mathrm{Glu}+\mathrm{Gln})(2.46 \mathrm{ppm})$ & 2 & 2.1 & 2 & 2.1 & 2.1 \\
\hline $\mathrm{mI}_{1} \mathrm{Tau}_{1}(3.26 \mathrm{ppm})$ & 2 & 2.2 & 2 & 2.2 & 2.2 \\
\hline $\mathrm{mI}_{2}(3.53 \mathrm{ppm})$ & 2 & 2.1 & 2 & 2.1 & 2.1 \\
\hline $\mathrm{Tau}_{2}$ (3.42ppm) & 1 & 1.5 & 1 & 1.5 & 1.5 \\
\hline $\mathrm{Ala}_{2}$ (3.78ppm) & 2 & 1 & 2 & 1 & 1 \\
\hline
\end{tabular}
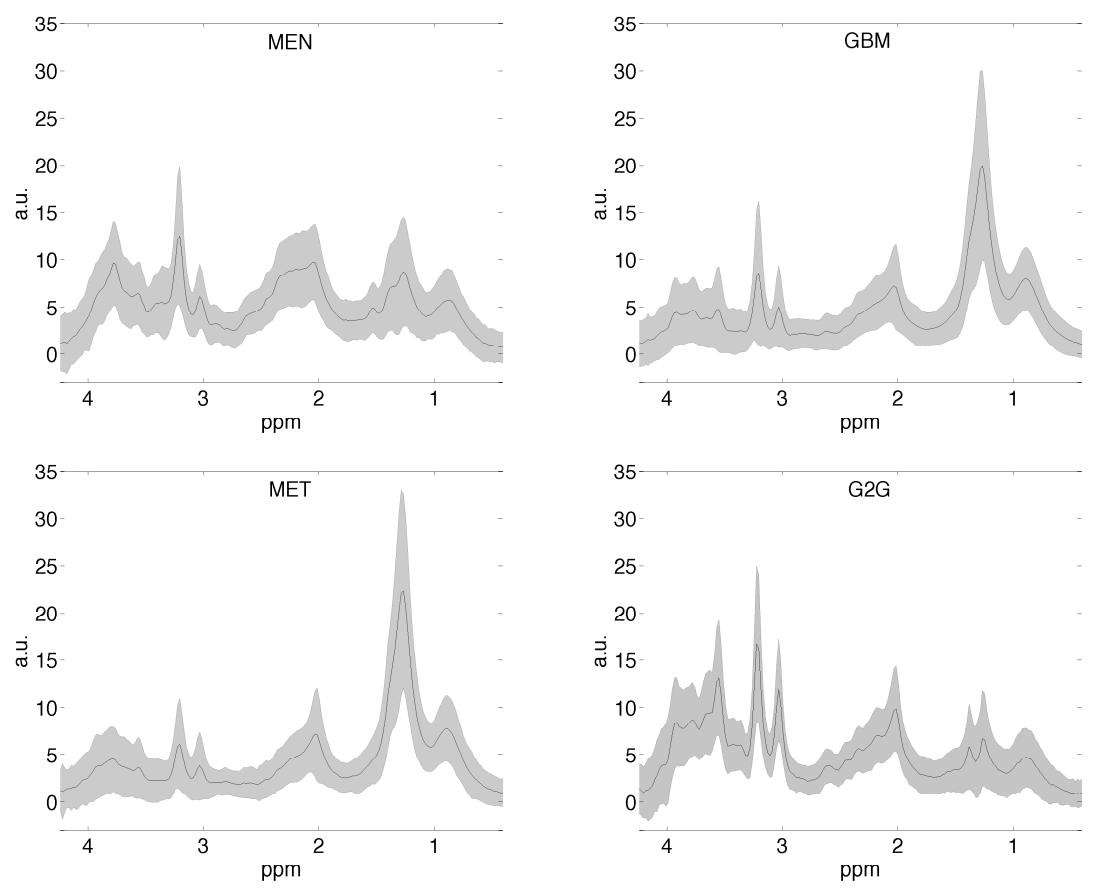

Figure 42: Mean and deviation of the four real spectra classes. 

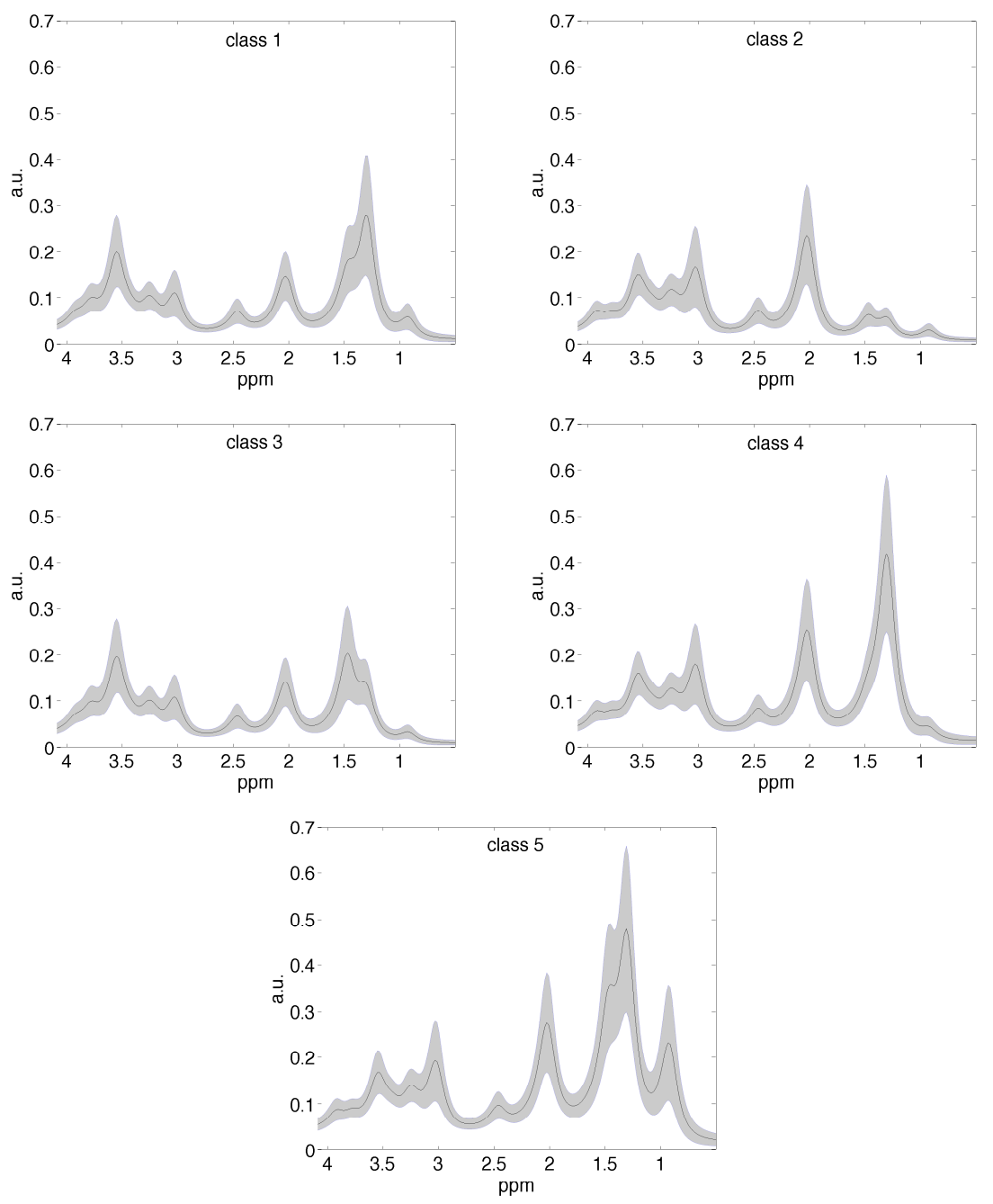

Figure 43: Mean and deviation of the five simulated spectra classes. 


\section{Noise addition}

The second artifact added to the synthesized dataset was noise. This term simulates the thermal noise of the samples and noise from the electronic components. To add noise to the spectra of the ideal dataset, the following expression (Equation 61) was used:

$$
s_{\text {noisy }}=s_{\text {ideal }}+\max \left(s_{\text {ideal }}\right) \cdot e_{n}
$$

where $e_{n}$ represents a Gaussian noise signal of zero mean and deviation $\sigma$. Following the definition of SNR used in the quality control defined in INTERPRET EU project and introduced in [EBW87], SNR was computed as the ratio of the highest signal intensity in the range $0-3.4 \mathrm{ppm}$ and the standard deviation of the noise in the range 9-11 ppm of the water suppressed spectrum. Therefore, $\sigma$ can be seen as the inverse of the minimum signal-tonoise ratio (SNR) of the dataset.

Five different datasets with different noise levels were simulated ranging from $\sigma=0$ to $\sigma=0.1$, that is, from a dataset without noise to a dataset with a minimum SNR equal to 10 . The noisiest spectrum considered here corresponds to the minimum SNR required by the quality control protocol of the INTERPRET and eTUMOUR EU projects [VdGJSH ${ }^{+} 08$ ] (SNR > 10), and, therefore, for the multicenter real dataset used in this work.

\section{Frequency shift}

The last artifact introduced was frequency shift. We simulated a typical spectra misregistration, wich was defined by:

$$
s_{\text {shift }}(t)=s_{\text {ideal }}(t+\delta \cdot U(-1,1)),
$$

where $\delta$ represents the ppm displacement of the spectrum with respect to the aligned one multiplied by a random value between -1 and 1 . Five datasets with different degrees of shifting artifact were calculated ranging from $\delta=0$ ppm to $\delta=0.1 \mathrm{ppm}$ of maximum shift.

\subsubsection{Real multicenter brain tumor MRS dataset}

Two real MRS datasets were used in our study. These datasets include four brain tumor classes consisting of CG2G, MET, MEN, and GBM. The first 1.5T ${ }^{1} \mathrm{H}$ SV-MRS dataset that was compiled during the INTERPRET EU project $\left[\mathrm{TUA}^{+} 06, \mathrm{JSAM}^{+} 06\right]$ and the second dataset is part of the $1.5 \mathrm{~T}$ dataset compiled during the eTUMOUR EU project [The07]. All the datasets were obtained using the magnetic resonance scanners of three major manufacturers (Philips, General Electric, and Siemens) in ten international centers. The 
number of cases used in each dataset is shown in Table 10. Two experiments have been performed using each of these datasets for training and testing respectively.

The INTERPRET dataset included 161 1.5T short TE SV-MRS spectra. The acquisition protocols included PRESS or STEAM sequences with the following spectral parameters already described in previous studies $\left[\mathrm{TMM}^{+} 03\right]$ : Repetition Time (TR) of $1600-2020 \mathrm{~ms}$; echo time (TE) of 20 or 30-32ms; spectral width (SW) of $1000-2500 \mathrm{~Hz}, 512,1024$, or 2048 data-points. All these cases were validated using a standard quality control protocol carried out by the INTERPRET Clinical Data Validation Committee and expert spectroscopists [TUA ${ }^{+} 06, \mathrm{VdGJSH}^{+} 08$ ], and all of them had a histopathological diagnosis.

The eTUMOUR dataset included 444 1.5T short TE SV-MRS spectra. These spectra were validated and histopathologically diagnosed by a panel of expert spectroscopists [TUA ${ }^{+} 06, \mathrm{VdGJSH}^{+} 08$ ] . The acquisition protocols included PRESS and STEAM with spectral parameters: TR of 1500-2000ms; TE of $30-31 \mathrm{~ms}$; SW of $500-2500 \mathrm{~Hz}$ and 512, 1024, or 2048 data-points.

The mean and deviation of the four real spectra classes included in the training and test datasets are presented in Figure 42.

\section{Preprocessing pipeline}

The multicenter dataset needs to be preprocessed before being used for classification purposes. In order to ensure the compatibility of our results with the ones in the literature, we selected a commonly used MRS processing pipeline defined in the INTERPRET and eTUMOUR EC projects. This processing pipeline was also included in the CDSSs software presented at $\left[\mathrm{SGGV}^{+} 08, \mathrm{PRJSM}^{+} 10, \mathrm{TUA}^{+} 06\right]$, and semi-automatically preprocesses the entire spectra dataset. A detailed description of this pipeline can be seen on Section 3.2.

The software used to preprocess the spectra was jMRUI 3.0 [ $\mathrm{VVV}^{+}$96] (for eddy current correction, phase correction, water suppression, apodization, zero filling, and baseline subtraction) and jDMS [PRJSM ${ }^{+}$10] (for normalization, alignment, and subsampling).

\subsection{FUNCTIONAL DATA ANALYSIS BASED ON REGION SEGMEN- TATION}

The RSFDA uses the highest scored spectrum points obtained by FC as seeds to define highly correlated regions by means of a growing strategy based on correlation. Once these regions have been defined, they are parameterized by means of the functional approach in order to define the extracted features. In a functional approach, the regions are characterized by the coefficient space. 


\subsubsection{Spectra processing}

A simple smoothing algorithm based on a moving average with a 5 point span was performed as a previous step to the spectra analysis. This process reduces the possibility of an early-stopping in the region growing process because of high noise.

\section{Fisher criterion}

The FC method [Fis36] is applied in the context of a classification problem and ranks the discriminative power for each variable by assigning a score value. The score depends on the ability of a variable to separate different classes while keeping a small within-class variance. The FC criterion for feature or spectra point is as follows:

$$
F C=\frac{\sum_{i=1}^{c} n_{i}\left(\mu_{i}-\mu\right)^{2}}{\sum_{i=1}^{c} n_{i} \sigma_{i}^{2}}
$$

where FC denotes the Fisher Score value, $\mathrm{c}$ denotes the number of classes in the dataset, $\mu$ denotes the global mean value, $\mu_{i}$ denotes the mean value in class $i, n_{i}$ denotes the number of cases in class $i$, and $\sigma_{i}^{2}$ denotes the variance in class $i$.

In the specific case of MRS data, FC assigns an FC value to each point of the spectra. A spectrum point will have a high score if it has high between-class scatter and low within-class scatter. In this sense, FC value can be seen as an indicator of the discriminant power of each individual point of the MRS spectrum.

In Figures 44 and 45, the seeds obtained for each of the six real binary classification problems considered are depicted as small circles in the diagonal of the autocorrelation matrix.

\subsubsection{Generation of seeds}

For each classification problem, the RSFDA uses the scoring values obtained by FC as seeds to define regions. These give a ordered relationship of the most potentially discriminant points for the available classes and, therefore, a sorted relation of possible seeds for the use in our method. The number of seeds has been established by Equation 64, where all points whose scores are over a specific threshold $\gamma$ are included. This threshold value $\gamma$ is defined by the maximum score value multiplied by a coefficient $\theta$ in the interval $[0,1]$. The value of $\theta$ have been determined in the training step using a validation dataset or by means of a tuning procedure.

$$
\text { score }>\gamma: \gamma=\theta \cdot \text { score }_{\max },
$$



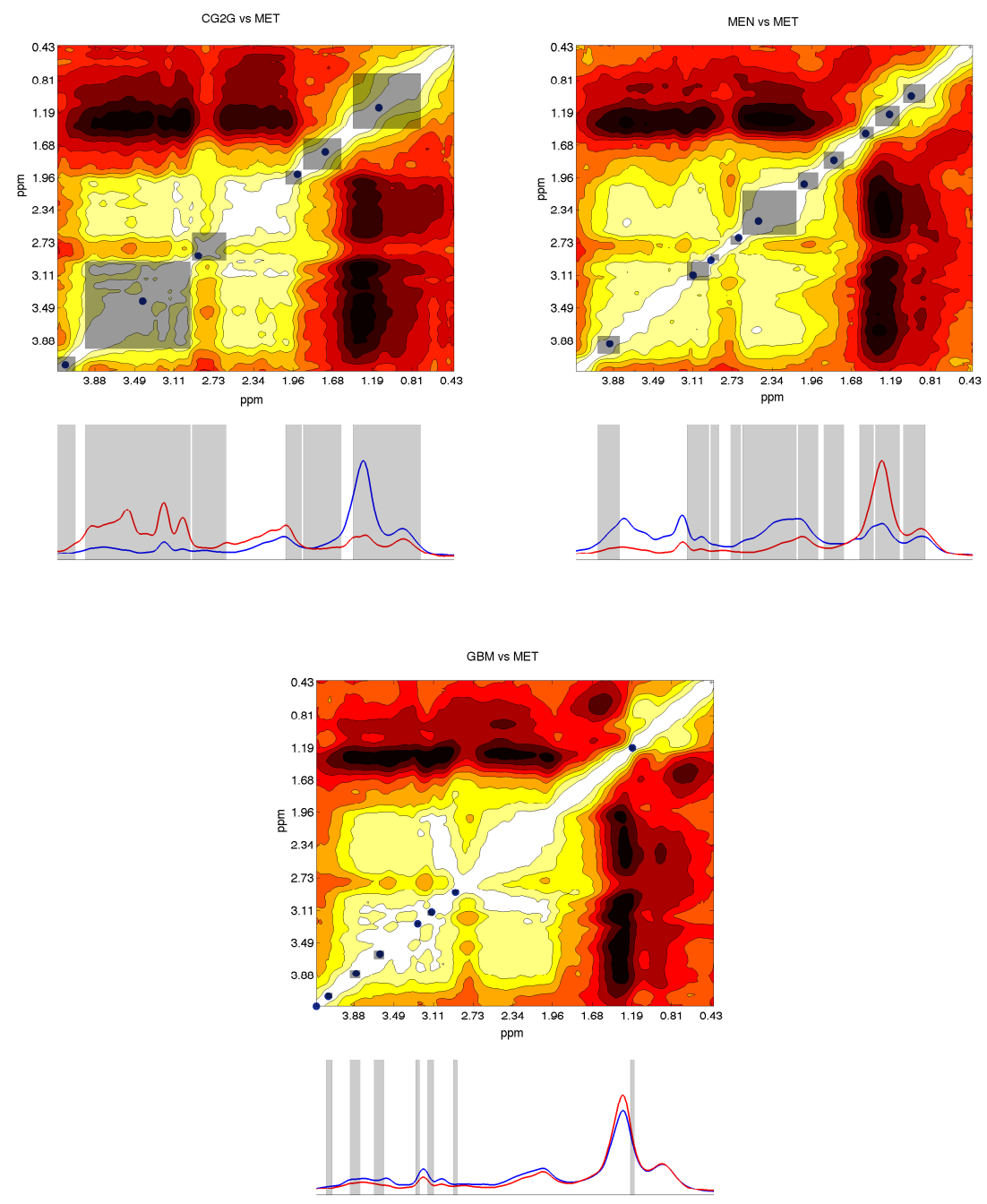

Figure 44: Autocorrelation contour plot and mean spectra for CG2G vs MET, MEN vs MET and GBM vs MET binary classification problems. The seeds used are depicted as small circles over the diagonal of the correlation matrix and the discriminant regions obtained are shown as shaded zones. In the autocorrelation contour plot darker colors represent a higher correlation while lower correlations are represented by lighter colors. 

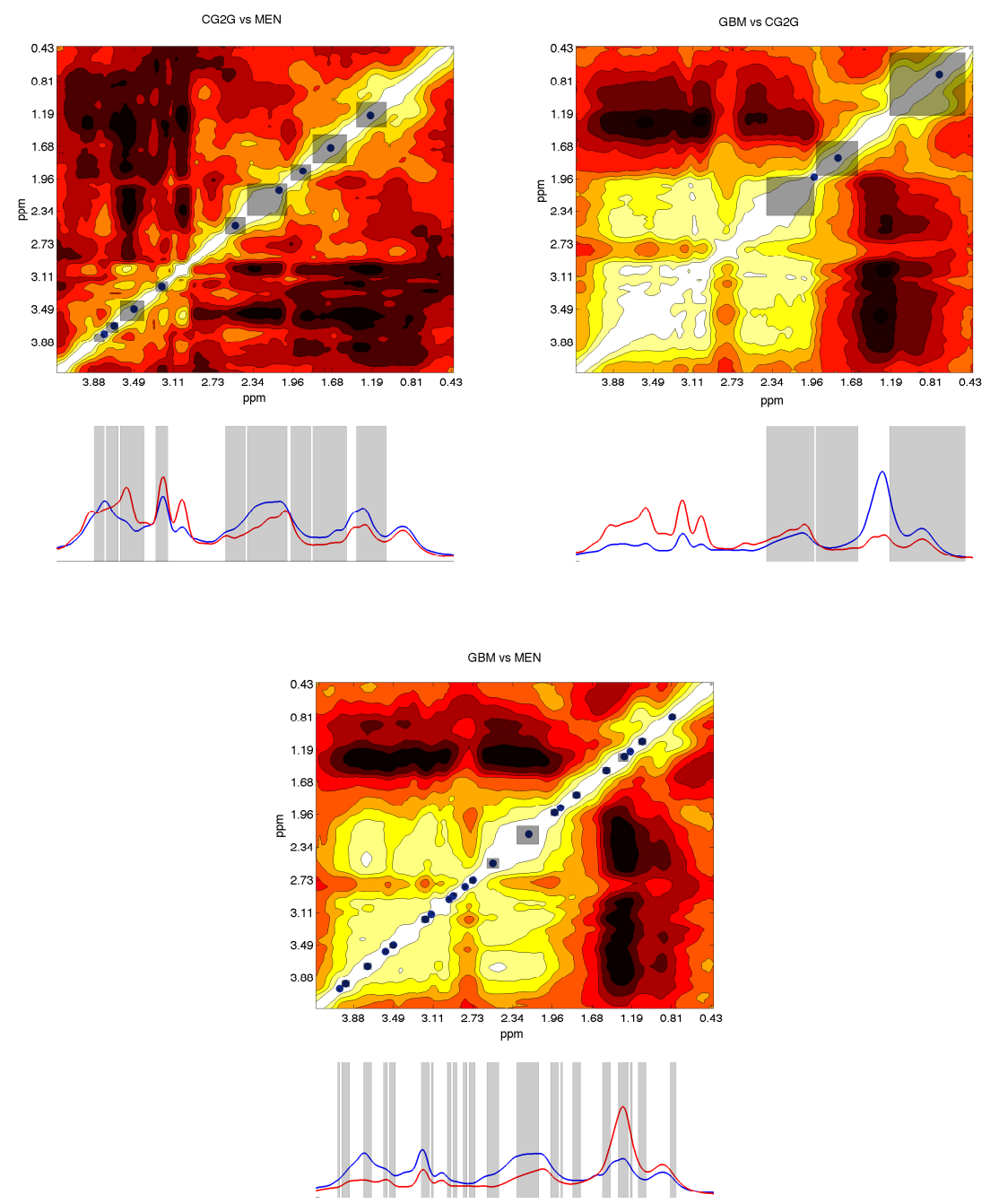

Figure 45: Autocorrelation contour plot and mean spectra for CG2G vs MEN, GBM vs CG2G and GBM vs MEN binary classification problems. The seeds used are depicted as small circles over the diagonal of the correlation matrix and the discriminant regions obtained are shown as shaded zones. In the autocorrelation contour plot darker colors represent a higher correlation while lower correlations are represented by lighter colors. 


\subsubsection{Region segmentation}

Due to the inherent high correlation between closed points in a continuos signal (which is typical in non i.i.d. random variables), if a point $s(f)$ in the spectra obtains a high score, it is highly probable that points in the $\epsilon$ interval around the point $f$ obtain a similar score value, too. To avoid this redundancy, our approach substitutes the point-by-point scoring by the region concept.

The first region is obtained using the first seed (the spectrum point with the highest FC score). Next, a growing strategy is applied by the inclusion in the region of the contiguous points whose correlation with the seed is greater than a fixed threshold $\tau$. All the points included in the first regions will be deleted from the seeds list. We repeat the same procedure successively with the remaining seeds until none is left. The value of $\tau$ has been determined in the training step using a validation dataset.

This methodology is described in algorithm 1, and the results obtained for each of the six real binary classification problems considered are shown in Figures 44 and 45.

It is important to notice that this algorithm is based in the assumption of connected regions, and therefore when a metabolite resonates in more than a single frequency, RSFDA will create two separate regions instead of a single disconnected one.

\subsubsection{Region modeling with functional description}

Once we have defined a set of potential discriminant regions, we model the spectra by means of a functional description of those regions.

The functional description is based on B-spline functions (a compact support basis functions developed by de Boor [Deb78]). Spline functions are the most common choice of approximation system for non-periodic functional data or parameters [RS05], and every spline function of a given degree, smoothness and domain partition can be represented as a linear combination of B-splines in the same grade and smoothness, and on the same partition [Deb78].

RSFDA uses a non-uniform B-splines basis, that is, the knots are not regularly distributed. Instead, they are established at each end of every obtained region. Therefore, if we use a B-spline basis of level $n$, we are approximating each region by a polynomial spline of $n-1$ degree, ensuring continuity and differentiability to the order $n-2$ in the points of connection between regions (in this case the knots). Figure 46 shows the B-splines basis used for GBM vs MET classification problem $(\mathrm{n}=7)$.

Once we have defined the functional basis to describe our spectra, we have to fit our data with the B-spline model (see Apendix A for a detailed description). To do so, we use the smoothing algorithm described by Ramsay et al in [RS05]. This method estimates a curve $x$ from observations $s_{i}=x\left(t_{i}\right)+\epsilon_{i}$. 


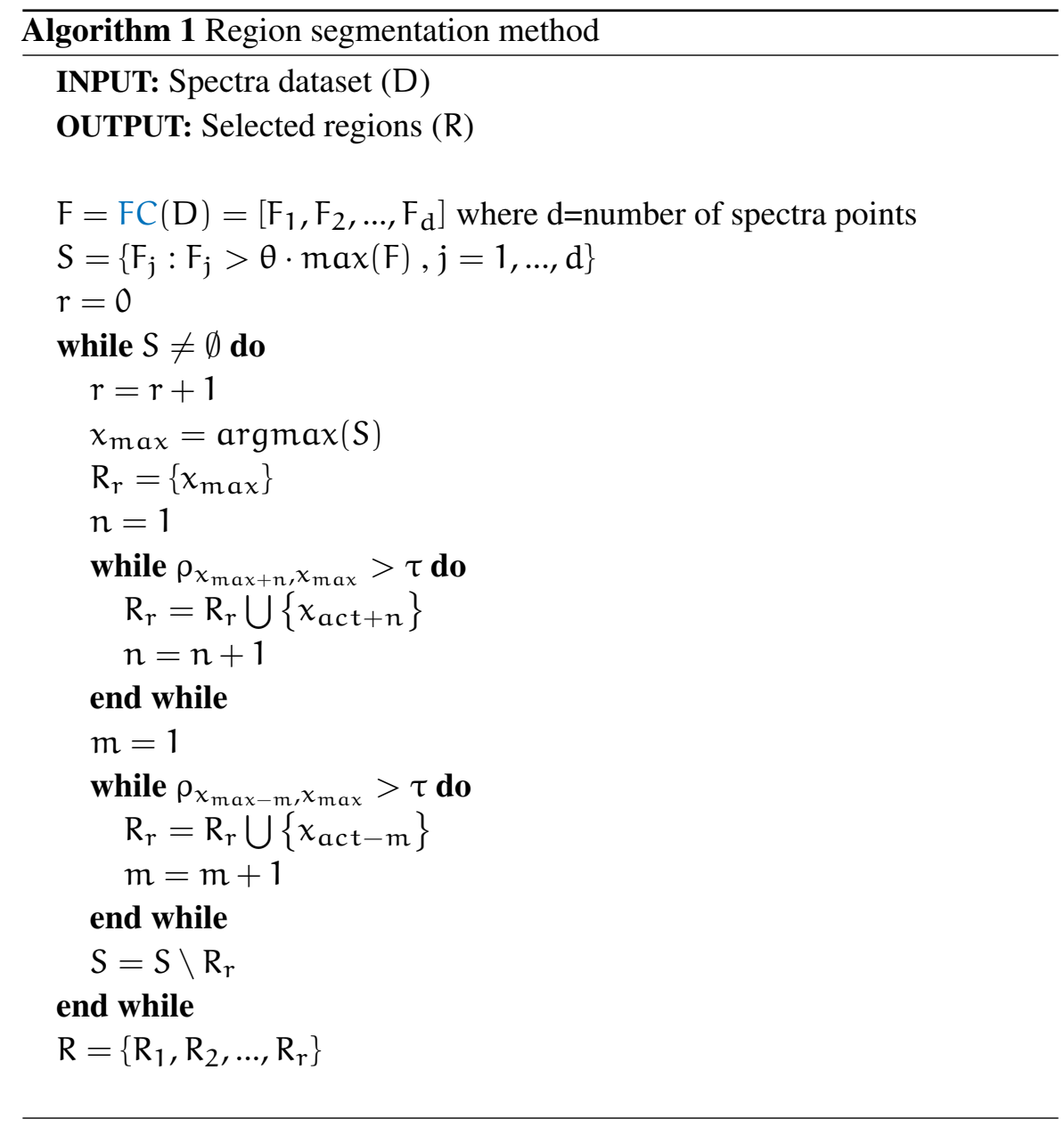

To avoid overfitting, it introduces a roughness penalty to the least-square criterion used for fitting the observations, resulting in a Penalized Least Squares Criterion (PENSSE):

$$
\operatorname{PENSSE}_{\lambda}(x)=\sum_{i=1}^{\text {length }(s)}\left(s_{i}-x\left(t_{i}\right)\right)^{2}+\lambda J(x),
$$

where $J(x)$ is a measure of roughness of $x$, and $\lambda$ is a coefficient that controls the amount of penalty introduced due to roughness of $x$. Higher $\lambda$ values mean a smoother model, and lower $\lambda$ values mean better fitting.

In order to define a measure of roughness $J$, we have used the concept of curvature or squared second derivative $\left(D^{2} x(t)\right)^{2}$ of a function [RS05]. Consequently, the measure of a function's roughness is the integrated squared second derivative

$$
J(x)=\int\left(D^{2} x(t)\right)^{2} d t
$$

As shown on 65 , the smoothing depends on the $\lambda$ parameter chosen. In order to automatically select a optimum $\lambda$ value for a specific dataset, 
the Generalized Cross-Validation measure developed by Craven and Wahba [CW79] has been used.

Finally, the B-spline coefficients obtained as a result of the fitting constitute the set of features extracted by the use of RSFDA for each specific MRS spectrum. It is important to notice that the B-splines used are compact support functions (localized) defined by the position of the knots and their degree. See Apendix A for a detailed description of how this coefficients (extracted features) are computed. The whole spectrum is modeled using RSFDA, considering different fitting precisions in each spectra zone depending on their discrimination power and correlation between points on the zone. For illustrative purposes, Figures 47 and 48 show the mean spectra of each of the classes used in each classification problem and their associated RSFDA based representation.

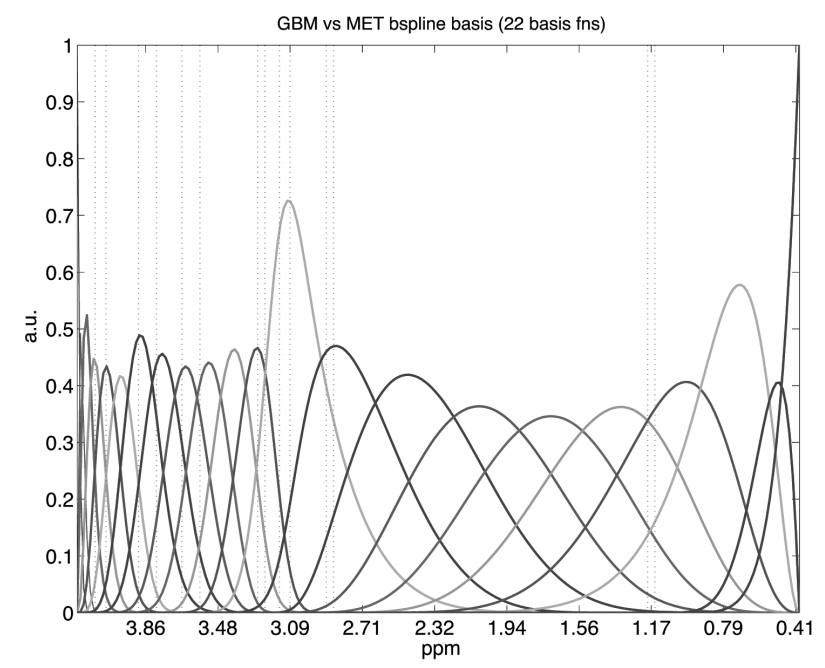

Figure 46: Example of the B-spline basis used for GBM vs MET classification problem considered. The positions of the knots defined by RSFDA are shown as vertical dashed lines.

\subsection{EXPERIMENTAL STRATEGIES}

To evaluate RSFDA, binary MRS classification problems have been considered. The performance obtained in these classification problems were compared with the performance obtained with other FE techniques: PCA [Pea01], since it is a FE method that is widely used in the literature, and FC [Fis36], since it is the starting point of the method presented. A standard LDA classifier and KNN were used to solve the different binary classification problems based on the output of the three different FE methods [Fis36].

Firstly, this methodology was applied on 15 simulated datasets consisting of 75.000 spectra (5000 spectra per dataset). For each artifact (noise, baseline 

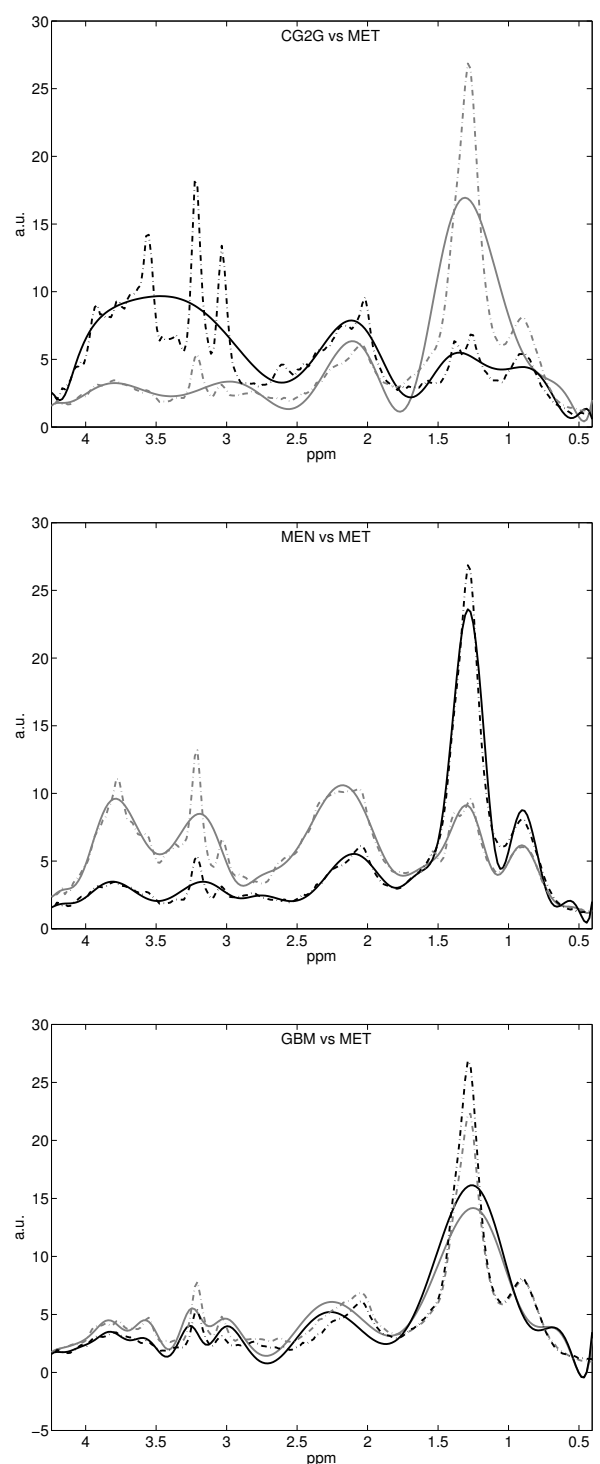

Figure 47: Original mean spectra for the two classes (dotted lines) and their reconstruction using RSFDA (solid lines) for the first three binary classification problems considered: CG2G vs MET, MEN vs MET and GBM vs MET 

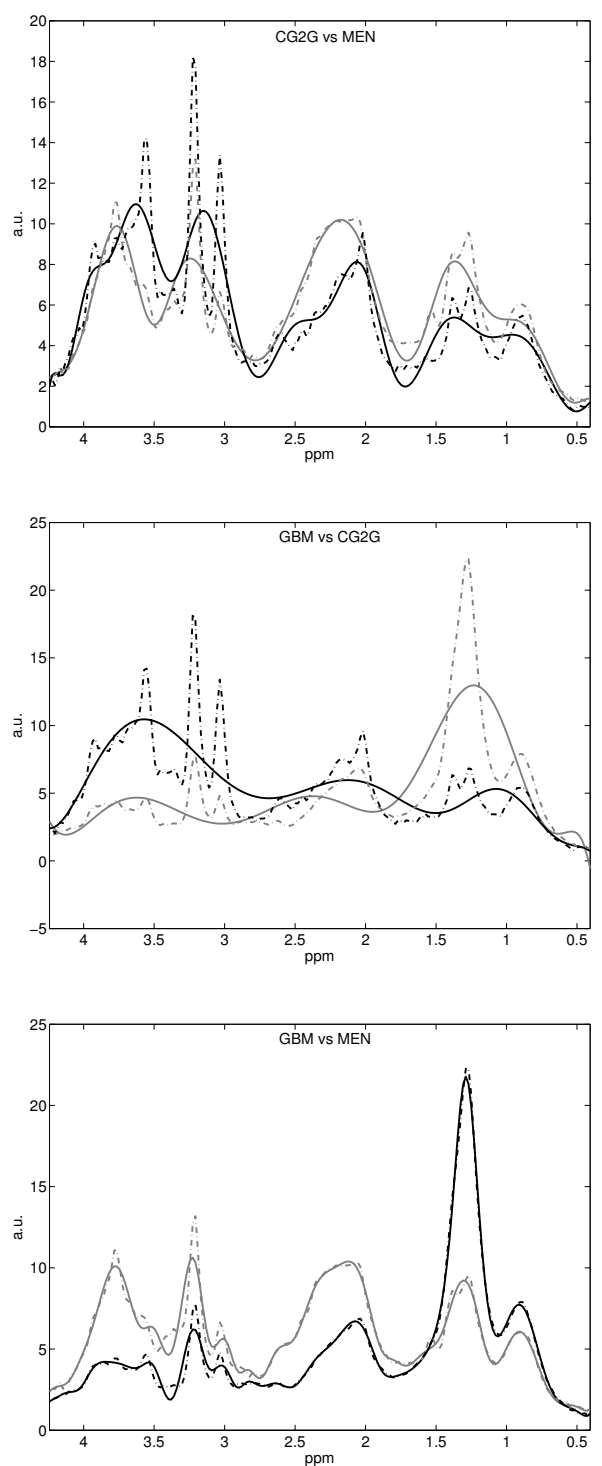

Figure 48: Original mean spectra for the two classes (dotted lines) and their reconstruction using RSFDA (solid lines) for the last three binary classification problems considered:CG2G vs MEN, GBM vs CG2G and GBM vs MEN. 
distortion, frequency shift), 5 datasets were simulated containing different levels of artifact in order to evaluate the behavior of each FE method under different conditions. A random sampling train-test was used for each of the datasets; $70 \%$ of the simulated dataset was used for training, $15 \%$ for $\theta$ and $\tau$ parameter estimation, and a $15 \%$ for test. This procedure was repeated in 5 rounds and was applied over the ten binary classification problems obtained by combination of the five spectral classes simulated. The final results are the ones averaged over the rounds and over the ten classification problems. In this study, a KNN classifier was used to avoid a badly conditioned correlation matrix in LDA due to highly correlated features of FC.

Secondly, the same methods were applied on a real multicenter dataset in order to analyze their performance under clinical-like conditions. In this case, twenty rounds of random sampling train-test were used. Two experiments were designed. Firstly, The MRS data compiled throughout the INTERPRET project was used for training (217 spectra). $50 \%$ of the MRS data compiled throughout the eTUMOUR project was used for parameter estimation (261 spectra), and the other 50\% was used as an independent test set (randomly selected). Secondly, the same experiment was repeated but using the eTUMOUR dataset for training, 50\% of INTERPRET dataset for parameter estimation, and the remaining $50 \%$ of INTERPRET dataset as an independent test. The final results are the ones averaged over the rounds. A widely used LDA classifier was used in this study.

\subsubsection{Classification methods}

In this study, we have used LDA [Fis36] as the classification algorithm fed by the extracted features to solve the classification problems for the following reasons: it is widely used in these kinds of classification problems $\left[\mathrm{GGLJS}^{+} 09, \mathrm{GGTV}^{+} 08\right.$ ]; it has been implemented in different CDSSs $\left[\mathrm{SGGV}^{+} 08, \mathrm{TUA}^{+} 06, \mathrm{PRJSM}^{+} 10\right]$; and it has good performance compared to state-of-the-art methods [GGLJS ${ }^{+} 09$ ].

Fisher's LDA [Fis36] is a classification technique that finds the linear combination of features that best separates the classes of objects. It consists of a ratio between the difference of the projected means and a measure of dispersion of each class. This function is optimal when the distance between means is maximal and the inside class dispersion is minimal.

KNN ( $\mathrm{k}=12)$ classification algorithm has also been used for the experiments with simulated datasets. In these datasets the badly conditioned correlation matrix of the features extracted by FC does not allow the use of LDA for the comparison among methods. This is due to the highly correlated features obtained by FC in the case of continuous signals. The KNN algorithm is an instance-based method for classifying objects based on the closest training examples in the feature space given a metric. 


\subsubsection{FE methods used for comparison}

We selected the PCA, and FC FE to compare with RSFDA. PCA linearly transforms the initial variables, so the first transformed variables describe the main variability of the signal. FC gives us a scored list of the initial variables, so the first variables in the score are more discriminant between the classes presented.

To define the number of variables to be considered from PCA, the percentage $\delta$ of variability expressed by the eigenvalues is usually used [Jol02]. To facilitate the comparison with the different FE methods, we considered two different conditions: the number of principal components that describe $70 \%$ of variability and $80 \%$ as suggested by Devos et. al at [DLS $\left.{ }^{+} 04\right]$.

To define the number of variables to be considered from FC, we used the condition described in the equation below:

$$
\text { score }>\delta \cdot \text { score }_{\max },
$$

To facilitate the comparison with the different FE methods, we considered two different conditions: the number of f-scores obtained for $\delta=0.65$ and for $\delta=0.80$.

Additionally, in the case of real multicenter datasets, we have compared the performance of RSFDA, not only with PCA and FC, but also with PI, Wavelet transform (WAV)+PCA, and ICA. WAV+PCA linearly transforms the wavelet coefficients [Dau92] obtained from the spectrum, so the first transformed variables describe the main variability of the signal. In this study a Daubechies 3 wavelet basis with a five level decomposition was used. ICA [HKO01] is a widely used technique for performing blind source separation. ICA is able to generate a basis of signals (sources), from a signal dataset in such a way that the regularity of each basis signal is maximized, and the statistical independence between the signals is maximized. In this study the number of ICs used is equal to the number of features defined by RSFDA.

\subsubsection{Evaluation metrics}

To determine the performance of a classifier, two evaluation metrics, ACC and $\mathrm{G}$, described in section 2.5.4 were used.

\subsection{RESULTS}

The results are presented in two main blocks: we present the results obtained with simulated data under different conditions of noise, frequency shifts, and baseline distortions. Then, we present the results obtained with the multicenter dataset. 


\subsubsection{Simulated MRS dataset}

\section{Effect of baseline distortions on FE performance}

In order to study the effect of baseline distortions on FE performance, an ideal dataset was considered, and different levels of baseline distortions where added. By using $\zeta$ parameters (see Equation 60) [0, 0.17, 0.35, 0.52, 0.7], respectively, we obtain a total of five datasets with different levels of baseline distortions. Figure 49 shows the results obtained.

The simulation shows that PCA underestimates the number of variables needed, achieving lower accuracies than FC and RSFDA, particularly in the case of high baseline distortion levels. This could be because most of the variance explained by the first PCA components is provided by the baseline and not from the metabolites. In the case of FC (scores $>\max _{\text {score }} \cdot 0.65$ ), the results obtained are similar to those obtained using RSFDA. However, FC (scores $>\max _{\text {score }} \cdot 0.80$ ) uses a higher number of variables to achieve the same ACC as RSFDA.

\section{Effect of noise on FE performance}

In order to evaluate the noise influence on the performance of the methods included in the study, five datasets were simulated with $\sigma$ values (see Equation 61) of $\{0,0.25,0.50,0.75,0.1\}$, respectively. Figure 50 shows the results obtained.

In the classification results obtained, PCA does not exhibit consistent behavior under noise variation. For noisy datasets the number of variables increases linearly for PCA, saturating in ACC. For the less noisy datasets PCA seems to underestimate the number of variables needed, resulting in low performances. Both the FC method and the RSFDA exhibit more stable behavior against noise in terms of number of selected variables. They also achieve good performance values in low noise datasets.

\section{Effect of frequency shift on FE performance}

The behavior of the FE methods against frequency shift artifacts was analyzed. Five datasets were simulated with a maximum shift of $[0,0.025,0.05,0.075,0.1]$, respectively. Figure 51 shows the results obtained.

In this last characterization, it can be observed that PCA performs well although a low number of variables or principal components are used. Both RSFDA and FC produce a better performance than PCA, although using 3 to 4 times the number of variables required by PCA. In the case of high frequency shift artifacts, FC achieve the best results. 

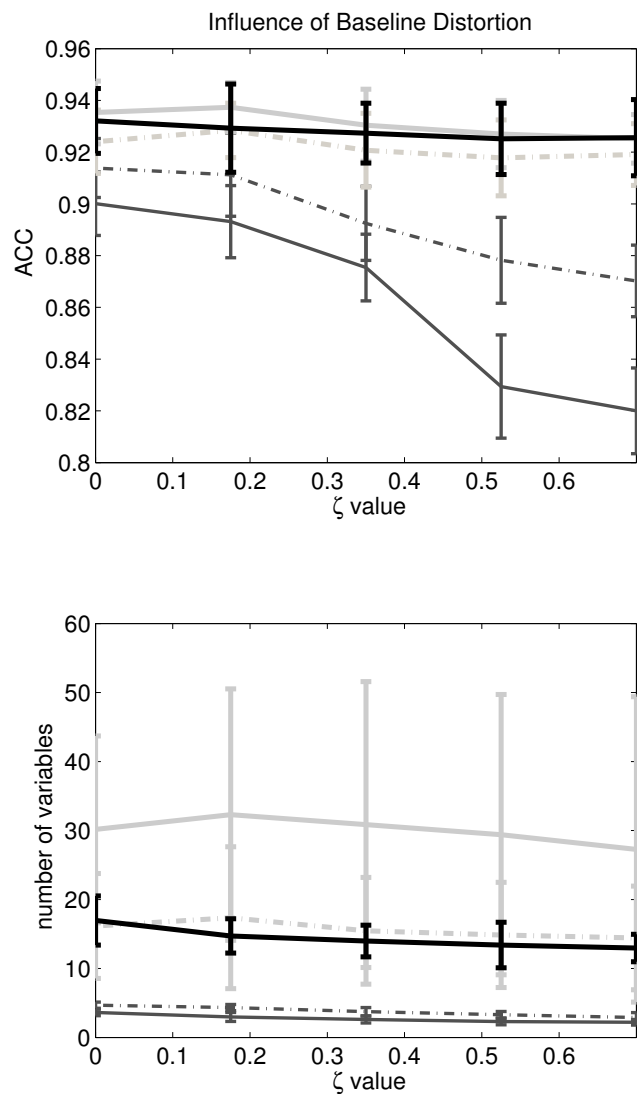

$$
\begin{aligned}
& \text { - - . . PCA (var<80) } \\
& \text { - PCA (var<70) } \\
& =-\mathrm{F} \text { - } \mathrm{F} \text {-score }\left(\text { scores }>\max ^{\star} 0.80\right. \text { ) } \\
& \text { F-score (scores>max*0.65) } \\
& \text { - RSFDA }
\end{aligned}
$$

Figure 49: Accuracy (ACC) and number of variables obtained in the classification problems using RSFDA, FC, and PCA feature extraction algorithms, with different datasets with different baseline distortion levels. 

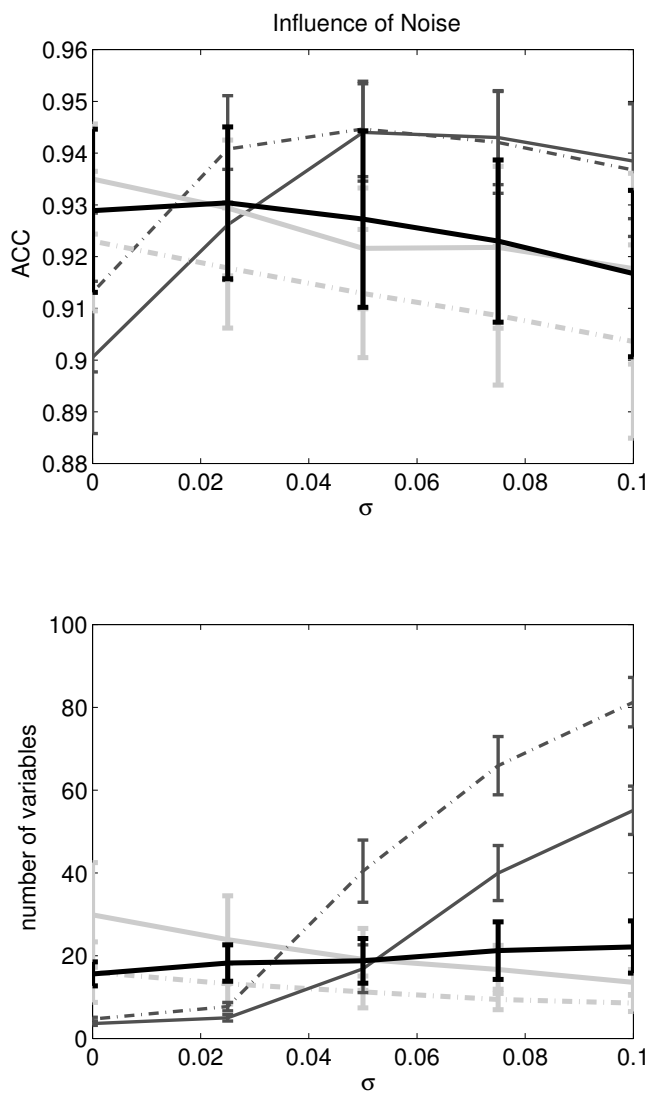

$$
\begin{array}{|l|}
- \\
- \text { - PCA }(\text { var }<80) \\
\text { PCA }(\text { var }<70) \\
\text { F-score }\left(\text { scores }>\max ^{*} 0.80\right) \\
\text { F-score }\left(\text { scores }>\max ^{*} 0.65\right) \\
\text { RSFDA }
\end{array}
$$

Figure 50: Accuracy (ACC) and number of variables obtained in the classification problems using RSFDA, FC, and PCA feature extraction algorithms, with different datasets with different noise levels. 

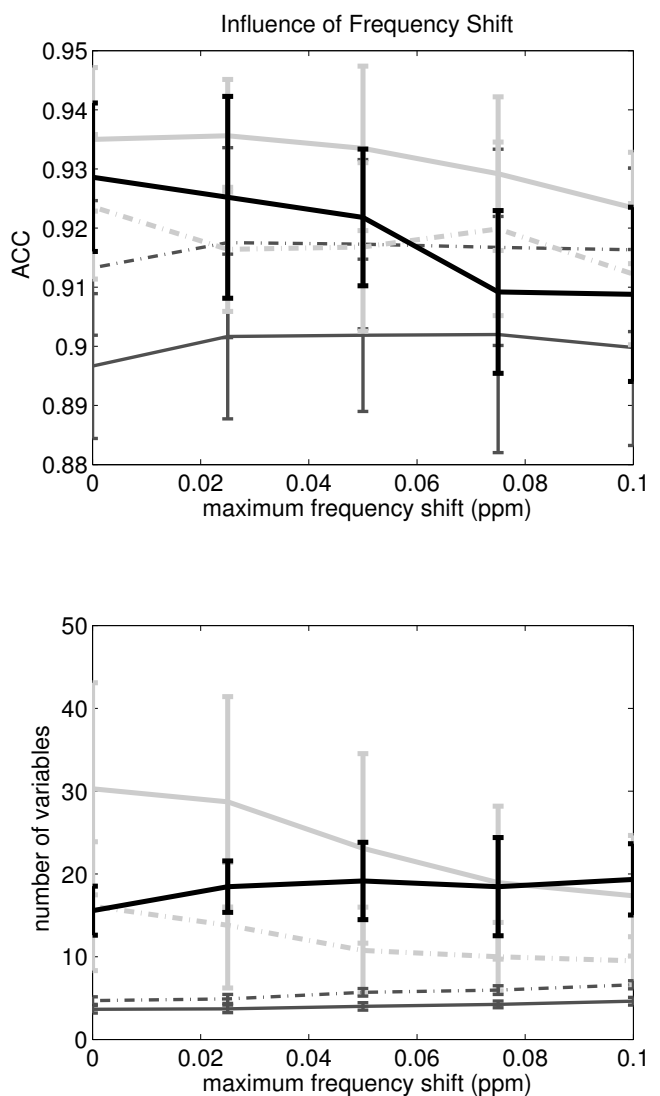

$$
\begin{aligned}
& \text { - - - PCA }(\text { var }<80) \\
& \text { - PCA (var<70) } \\
& =-\mathrm{F} \text { - } \mathrm{F} \text {-score }\left(\text { scores }>\max ^{\star} 0.80\right) \\
& \text { F-score (scores }>\text { max }^{*} 0.65 \text { ) } \\
& \text { - RSFDA }
\end{aligned}
$$

Figure 51: Accuracy (ACC) and number of variables obtained in the classification problems using RSFDA, FC, and PCA feature extraction algorithms, with different datasets with different frequency shifts. 


\subsubsection{Multicenter brain tumor MRS dataset}

Following the simulated datasets, we analyzed the behavior of the FE methods on a real muticenter dataset.

Figures 44 and 45 show an autocorrelation matrix plot for each of the six datasets used in the binary classification problems for the evaluation of the FE methods. Shaded areas show the segmented regions for the RSFDA analysis. The set of seeds used for the region segmentation are depicted as small circles over the diagonal of the matrix. The mean spectra for each of the classes are shown below the autocorrelation plot.

Figure 46 shows the B-spline basis defined by the RSFDA algorithm for GBM vs MET classification problem. The positions of knots defined by RSFDA are depicted as vertical dashed lines.

Once the region segmentation has been done and the B-spline basis is defined, we can calculate a functional representation of the spectra (see Figures 47 and 48). It is important to note that these representations are not optimized to accurately describe the spectra shape, but to highlight discriminant features of the spectra.

Table 12 presents a comparison of the the classification results obtained by the different FE algorithms over 20 rounds, for the two experiments performed. We have described the performance of each method for each binary classification problem in terms of mean G (see Equation 40). The mean number of variables used by each of the methods are also presented. This table also includes the PCA and FC algorithms using the same number of variables as the ones defined for RSFDA. These results facilitate the comparison among the methods when the same number of variables are used.

\subsection{DISCUSSION}

The results obtained have shown that RSFDA is robust against the most common artifacts in MRS. The number of relevant regions obtained by RSFDA remains constant for different levels of noise, baseline distortion, or frequency shift. In contrast, PCA overestimates the number of principal components needed when a high level of noise is present on the MRS dataset and underestimates the number of principal components needed when baseline distortions affect the MRS dataset. This is due to the strategy based on the covariance explained when used on PCA. When a dataset is affected by noise, more principal components are needed to explain the same variance percentage without explaining more relevant information. In contrast, in the case of baseline distortions, few principal components contain a high variance percentage, which is mostly related to baseline and not to discriminant metabolic information. 
Table 12: The performance results in terms of $\mathrm{G} \pm \sigma$ for each of the six binary classification problems considered based on the multicenter brain tumor MRS datasets. In the first table, the results presented have been obtained training the classification model with INTERPRET dataset and testing them with eTUMOUR dataset. In the second table, the results presented have been obtained training the classification models with eTUMOUR dataset and testing them with INTERPRET dataset.

Training with INTERPRET dataset and testing with eTUMOUR dataset

\begin{tabular}{ccccccccc}
\hline FE method & $\begin{array}{c}\text { PCA } \\
(\text { var }<80)\end{array}$ & $\begin{array}{c}\text { PCA } \\
(\text { var }<70)\end{array}$ & $\begin{array}{c}\text { FC } \\
(\text { scores }>) \\
\text { max } 0.8)\end{array}$ & $\begin{array}{c}\text { FC } \\
(\text { scores }>) \\
\text { max } \cdot 0.65)\end{array}$ & RSFDA & PI & $\begin{array}{c}\text { ICA } \\
(\text { scores }=\end{array}$ & $\begin{array}{c}\text { WAV+PCA } \\
(\text { var }<80)\end{array}$ \\
GBM-MEN & $0.77 \pm 0.04$ & $0.74 \pm 0.04$ & $0.71 \pm 0.04$ & $0.72 \pm 0.04$ & $0.79 \pm 0.03$ & $0.81 \pm 0.02$ & $0.78 \pm 0.02$ & $0.77 \pm 0.02$ \\
GBM-CG2G & $0.80 \pm 0.03$ & $0.79 \pm 0.02$ & $0.78 \pm 0.03$ & $0.76 \pm 0.02$ & $0.80 \pm 0.03$ & $0.78 \pm 0.02$ & $0.76 \pm 0.01$ & $0.77 \pm 0.01$ \\
CG2G-MEN & $0.79 \pm 0.04$ & $0.77 \pm 0.04$ & $0.71 \pm 0.04$ & $0.69 \pm 0.04$ & $0.80 \pm 0.04$ & $0.78 \pm 0.02$ & $0.72 \pm 0.02$ & $0.72 \pm 0.02$ \\
GBM-MET & $0.49 \pm 0.04$ & $0.46 \pm 0.05$ & $0.47 \pm 0.05$ & $0.52 \pm 0.04$ & $0.52 \pm 0.05$ & $0.58 \pm 0.02$ & $0.58 \pm 0.01$ & $0.56 \pm 0.04$ \\
MEN-MET & $0.76 \pm 0.04$ & $0.76 \pm 0.04$ & $0.78 \pm 0.03$ & $0.83 \pm 0.03$ & $0.82 \pm 0.03$ & $0.78 \pm 0.02$ & $0.81 \pm 0.02$ & $0.80 \pm 0.02$ \\
CG2G-MET & $0.78 \pm 0.04$ & $0.76 \pm 0.04$ & $0.80 \pm 0.04$ & $0.82 \pm 0.03$ & $0.82 \pm 0.03$ & $0.84 \pm 0.02$ & $0.74 \pm 0.01$ & $0.81 \pm 0.01$ \\
\hline mean & 0.73 & 0.71 & 0.71 & 0.72 & 0.76 & 0.76 & 0.74 & 0.74 \\
\hline params & 9 & 5 & 8 & 17 & 19 & 15 & 19 & 9 \\
\hline
\end{tabular}

Training with eTUMOUR dataset and testing with INTERPRET dataset

\begin{tabular}{|c|c|c|c|c|c|c|c|c|}
\hline FE method & $\begin{array}{c}\text { PCA } \\
(\text { var }<80)\end{array}$ & $\begin{array}{c}\text { PCA } \\
(v a r<70)\end{array}$ & $\begin{array}{c}\text { FC } \\
(\text { scores }>) \\
\max \cdot 0.8)\end{array}$ & $\begin{array}{c}\mathrm{FC} \\
(\text { scores }>\text { ) } \\
\max \cdot 0.65)\end{array}$ & RSFDA & PI & $\begin{array}{c}\text { ICA } \\
(\text { scores }= \\
\text { RSFDA })\end{array}$ & $\begin{array}{l}\text { WAV+PCA } \\
(\text { var }<80)\end{array}$ \\
\hline GBM-MEN & $0.88 \pm 0.04$ & $0.89 \pm 0.03$ & $0.71 \pm 0.05$ & $0.83 \pm 0.04$ & $0.94 \pm 0.02$ & $0.91 \pm 0.02$ & $0.91 \pm 0.01$ & $0.91 \pm 0.02$ \\
\hline GBM-CG2G & $0.84 \pm 0.04$ & $0.83 \pm 0.05$ & $0.69 \pm 0.05$ & $0.83 \pm 0.03$ & $0.93 \pm 0.04$ & $0.88 \pm 0.02$ & $0.88 \pm 0.02$ & $0.88 \pm 0.02$ \\
\hline CG2G-MEN & $0.96 \pm 0.03$ & $0.84 \pm 0.05$ & $0.69 \pm 0.05$ & $0.73 \pm 0.04$ & $0.87 \pm 0.03$ & $0.92 \pm 0.03$ & $0.91 \pm 0.03$ & $0.91 \pm 0.03$ \\
\hline GBM-MET & $0.59 \pm 0.05$ & $0.58 \pm 0.06$ & $0.44 \pm 0.06$ & $0.54 \pm 0.06$ & $0.58 \pm 0.05$ & $0.57 \pm 0.02$ & $0.56 \pm 0.03$ & $0.52 \pm 0.02$ \\
\hline MEN-MET & $0.98 \pm 0.01$ & $0.94 \pm 0.02$ & $0.84 \pm 0.05$ & $0.91 \pm 0.03$ & $0.92 \pm 0.03$ & $0.96 \pm 0.01$ & $0.96 \pm 0.01$ & $0.96 \pm 0.01$ \\
\hline CG2G-MET & $0.93 \pm 0.03$ & $0.91 \pm 0.04$ & $0.84 \pm 0.04$ & $0.91 \pm 0.04$ & $0.97 \pm 0.02$ & $0.98 \pm 0.01$ & $0.98 \pm 0.01$ & $0.97 \pm 0.01$ \\
\hline mean & 0.86 & 0.83 & 0.70 & 0.79 & 0.87 & 0.87 & 0.87 & 0.86 \\
\hline params & 6 & 3 & 6 & 9 & 18 & 15 & 18 & 6 \\
\hline
\end{tabular}


Simultaneously, the performance results obtained in terms of ACC against noise and baseline distortion reveals that RSFDA could be an interesting alternative to the common FE methods used in MRS classification.

In the study of the influence of noise on performance, PCA achieved the highest ACC values for low SNR. However, it overestimated the number of variables needed in the case of high noise levels. Figure 50 shows where the ACC curve saturates close to the minimum SNR dataset while the number of variables used continues growing linearly with noise. When comparing RSFDA with FC (scores $>\max _{\text {score }} \cdot 0.65$ ), similar performances are obtained for all noise levels. However, FC (scores $>$ max $_{\text {score }} \cdot 0.65$ ) uses twice the number of variables used by RSFDA for high SNR values. For FC (scores $>$ max $_{\text {score }} \cdot 0.80$ ), it seems that it underestimates the number of variables needed, obtaining a lower performance from intermediate to low SNR.

In the case of baseline distortions, RSFDA performance was proven to be both high and stable. It is able to obtain the same ACC for datasets with different levels of baseline distortions without increasing the number of variables needed for the classification. In the case of PCA, performance decreases substantially when baseline distortions become important. This could be due to an underestimation of the number of variables used. In comparison, FC (scores $>\max _{\text {score }} \cdot 0.65$ ) tends to overestimate the number of variables needed without increasing the ACC.

Finally, in the study of the influence of frequency shift on performance, RSFDA performs better than PCA for small frequency shifts, but using a greater number of variables. For frequency shifts over $0.05 \mathrm{ppm}$, both methods obtain the same ACC but using a different number of variables (RSFDA needs more variables). FC uses a greater number of variables than RSFDA and PCA, but for frequency shifts above $0.025 \mathrm{ppm}$, it obtains a good relation between performance and number of variables.

The simulated study concludes that, although RSFDA achieves performances similar to PCA and FC, it has a competitive advantage in the case of strong baseline distortion datasets and also has strong stability against artifacts . It is important to note that in the case of PCA or FC, the results obtained with simulated datasets could have been slightly optimistic since the MRS simulations and these two methods are based on Gaussian distribution models. Finally, it is important to notice that although the simulations have taken into account baseline distortions, noise, and frequency shift artifacts, other effects as eddy currents, J-coupling effects, or stimulated echoes (as the results of short TE) have not been included in the simulation.

The simulated study allowed us to obtain a parametric study of the influence of different artifacts on the performance of each FE method. However it was necessary to study their behavior on real data for a more realistic problem that is closer to clinical practice. To do so a multicenter dataset containing 605 cases and four brain tumor types was analyzed. This work proposes ten 
binary classification problems as a result of all the possible combinations between classes.

Figures 44 and 45 show the different regions that have been selected by RSFDA for each of the real multicenter classification problems presented. The regions obtained in GBM vs MET classification problem are particularly significant. In this problem, narrow regions are obtained due to the inherent complexity of the discrimination, where both classes exhibit similar spectra shape and the more correlated regions of the spectra are not so discriminant between these two classes.

RSFDA ensures a optimum density of B-spline functions where the regions have been defined (see Figure 46). This means that RSFDA ensures a detailed description of the discriminant regions, while the rest of the spectra are described using only a few B-splines. This methodology attempts to reduce the number of descriptors to obtain a functional representation that is optimal for classification purposes. This can be clearly observed in the case of GBM vs MET (see Figure 46), where the density of B-spline functions is directly related to the regions defined in Figure 44. A very different case can be observed in the GBM vs CG2G problem, where only three regions were defined with large widths close to the distances between regions in the $[2.5,0.43]$ ppm range. In this range, the B-spline basis distribution is almost uniform, and, therefore, the refinement level of the spectra reconstruction is uniform throughout the entire range. Following the comparison of these two cases, on GBM vs MET a high level of detail in the reconstruction can be observed in the spectra zone between 4 and $2.7 \mathrm{ppm}$, allowing the identification of $\mathrm{Cr}$, Cho, and Gly peaks (among others) . However, in the same zone of the GBM vs CG2G case, no detail can be distinguished in the reconstruction. It is important to note that the mean number of features obtained by RSFDA is comparable to the number of metabolites considered by the radiologist (see Figure 49, 50, 51, and Table 12).

Finally, the classification results for the two experiments based on the multicenter datasets were obtained for each binary classification problem (see Table 12). In general terms, the results of both experiments show the expected behavior: 1) when the classification models were trained with a larger dataset (eTUMOUR dataset), the classification performances were substantially improved with respect the model trained with smaller dataset (INTERPRET dataset), around a 13\%. 2) The relations between performances obtained using different FE methods remained similar in both experiments, with the exception of FC (scores $>\max _{\text {score }} \cdot 0.80$ ) where the performance is slightly lower. In both experiments RSFDA, ICA, and PI got the best performance results, although PCA, and PCA+WAV also obtained good results. These results agree with those included in the following studies [GGLJS ${ }^{+}$09], and [DLS ${ }^{+}$04], which are based on similar datasets as the ones used in this work.

RSFDA obtains the best performances in GBM vs. CG2G classification problems in both experiments by using INTERPRET and eTUMOUR datasets as 
independent tests. RSFDA also obtains the best results in CG2G vs. MEN when testing with eTUMOUR dataset and in GBM vs. MEN when testing with INTERPRET dataset. In the cases of MEN vs. MET and CG2G vs. MET, RSFDA obtains the second best results when using eTUMOUR and INTERPRET dataset respectively for testing. Finally, for the classification of GBM vs. MET all methods have obtained low performance values as already reported in $\left[\mathrm{LPGG}^{+} 08\right]$, [GGLJS $\left.{ }^{+} 09\right]$ and [DLS $\left.{ }^{+} 04\right]$, due to its complexity. 
As has been introduced in section 2.4.1, in PET scanners, three kinds of coincidences could occur (Figure 21): (A) scattered events in which one or both $\gamma$ photons scatter before arriving to the detector; (B) random coincidences consisting of two $\gamma$ photons generated in different nuclei decays that are detected close enough in time to be coincidence; and (C) true coincidences, consisting of two $\gamma$ photons generated in the same nucleus decay and have not been scattered.

Random coincidences are one of the main sources of image degradation in PET. In conventional PET, coincidence identification is usually carried out through a coincidence electronic unit, so that randoms occur when two photons arising from two annihilation events are detected within the same coincidence time window. To compensate for random coincidences, the number of random events contributing to each Line-of-Response (LOR) should be estimated. On the other hand, some novel systems allow coincidences to be classified post-acquitisition in software, or in real time through a digital coincidence engine in a FPGA (Field Programmable Gate Array). These approaches provide the user maximum flexibility in the postprocessing of acquired data, thus allowing alternative coincidence sorting procedures to be applied. In this work a natural application of ANN methods to the problem of reducing random coincidences, has been investigated. It has been compared against a conventional coincidence sorting algorithm based on a time coincidence window combined with geometrical conditions. At matched efficiencies, the ANN based method presents always a sorted output with a smaller random fraction. In addition, two differential trends are found: the conventional method presents a maximum achievable efficiency while the ANN-based one is able to increase the efficiency up to unity, the ideal value, but at the cost of increasing the random fraction. Pattern recognition capabilities of ANN could be more adequate for more complex situations, where simple techniques such as the conventional sorter fails to model the underlying physics.

The results included in this chapter were presented in different international congresses and published as proceedings in [FGOTR08, FGOC ${ }^{+} 10 \mathrm{a}$, $\mathrm{FGOC}^{+}$10b]. 


\subsection{INTRODUCTION}

Some small animal PET scanner prototypes allow that all single events are recorded (singles list-mode), so that all coincidence events are sorted post-acquisition in software $\left[\mathrm{MHP}^{+} 05 \mathrm{a}, \mathrm{SBL}^{+} 05\right]$. Also the use of FPGAbased digital coincidence engines $\left[\mathrm{TVR}^{+} 08\right]$ offers a great flexibility for coincidence sorting in real time. These features open new possibilities to use alternative, more efficient coincidence sorting procedures

The most common coincidence sorting procedures are: (a) finding coincidences inside a time window [TERS ${ }^{+} 08$ ], or (b) using a logical "OR" between all pulse signals $\left[\mathrm{TLV}^{+} 05\right]$. However, the identification efficiency of both methods is not optimal. In fact, it is degraded by several effects such as: finite time resolution, deadtime, pile-up, etc. Therefore, both methods generate accidental coincidences (randoms), ie. coincidences made of two photon detections, which originate from different positron annihilations. Randoms are mainly a consequence of the finite time resolution of the device and constitute one of the main sources of image degradation in PET.

In this work, aimed at reducing the number of randoms within the sorted coincidence list, the new coincidence sorter based on ANN is defined and studied. The objective of this work is to asses the performance of the ANN approach, compared to a conventional sorting technique based on a time coincidence window combined with the application of geometrical conditions, and study the effects of both sorters on the reconstructed image. The ANN method uses a feed-forward neural network using as input variables of the model: temporal difference between two events, sum of their energies, distance between detection rings and angular distance between sectors for each coincidence (see Figure 52). To train the ANN, we have used simulated singles list-mode signals $\left[\mathrm{MHP}^{+} 05 \mathrm{~b}, \mathrm{Fah02}\right]$ of an extended cylindrical source of high activity $(1 \mathrm{mCi})$ in a small animal PET scanner, in order to increase the generalization capabilities of the trained model. For testing, Monte-Carlo simulated data of a generic small animal PET scanner have been generated for different phantoms and concentration activities.

\subsection{METHODS}

\subsubsection{Monte-Carlo simulations}

The simulation tool GATE was chosen. We have implemented a small animal PET scanner consisting of 8 axial rings of 20 modules each. Each module contains a $4 \times 1$ matrix of $2 \times 2 \times 8 \mathrm{~mm}^{3}$ Lutetium Oxyorthosilicate (LSO) crystals readout individually. The inner diameter is $8 \mathrm{~cm}$ and its axial length is $1.95 \mathrm{~cm}$. An energy resolution of $21 \%$ at $511 \mathrm{keV}$ and a time resolution of $10 \mathrm{~ns}$ are assumed. To avoid real multiple coincidences arising from intercrystal scatter, the low energy threshold was set at $400 \mathrm{keV}$. The high energy threshold was set to $750 \mathrm{keV}$. 


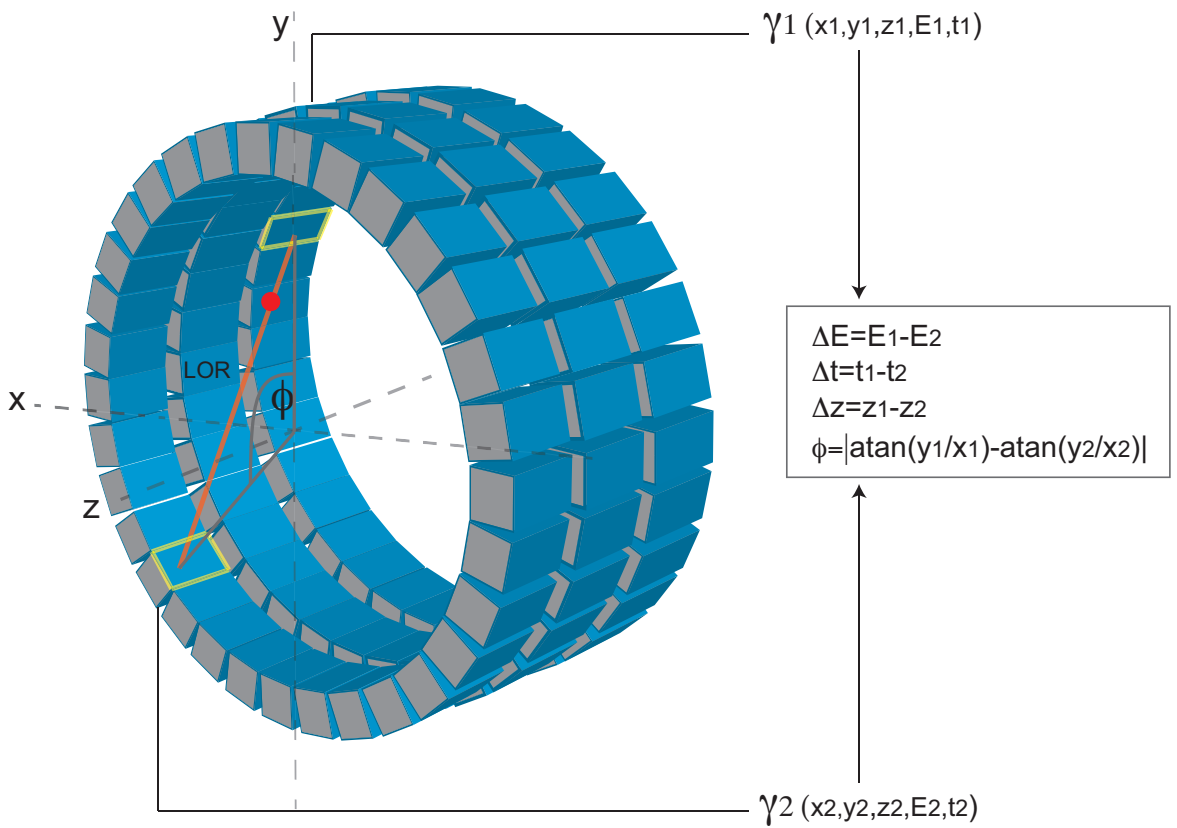

Figure 52: Input variables for the ANN based sorting method in a PET scanner consisting in 3 axial rings of 32 modules each .
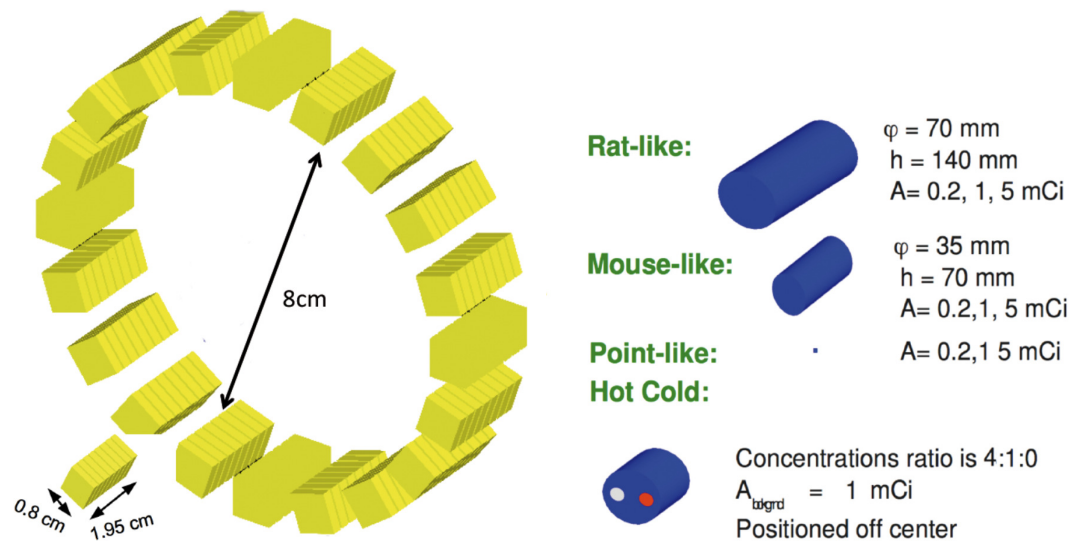

Figure 53: Schema of the simulated scanner used in this work (left side) the and phantoms used to evaluate the studied models (right side). 
To study the effect of the activity and source geometry in the performance of both coincidence sorters, several phantoms were simulated (see Figure 53): A point-like source (a centered small sphere, diameter: $2 \mathrm{~mm}$ ); a line source of length $35 \mathrm{~mm}$, placed off-centered and axially-oriented; a mouselike phantom, which is a centered cylinder of $35 \mathrm{~mm}$ of diameter and $70 \mathrm{~mm}$ of height, and a rat-like phantom (a centered cylinder, diameter: $70 \mathrm{~mm}$, and height: $140 \mathrm{~mm}$ ). For each phantom, three levels of activity were considered: $5 \mathrm{mCi}, 1 \mathrm{mCi}$ and $0.2 \mathrm{mCi}$. Additionally, a cylindrical phantom with two rod inserts was also simulated to evaluate the quality of the reconstructed image using both sorting methods. The concentration ratio between the hot, background and the cold regions was 4:1:0 respectively, and the total amount of activity was $1 \mathrm{mCi}$.

\subsubsection{Coincidence sorters}

We have implemented and compared the following methods:

ANN SORTER The objective of this ANN is to classify two given events into coincident or not coincident. The architecture of the network consisted of two hidden layers with four units in each layer, and a single processor in the output layer. The architecture of the network has been chosen by the evaluation of different network configurations when testing on an independent test dataset. The ANNs architectures tested include architectures with one hidden layer, with two and also without hidden layers. Different number of neurons per layer were also tested including 8,4 , and 2 neurons. The hidden units used an hyperbolic tangent activation function and the output units used a logistic activation function. To train the ANN, a homogeneous cylindrical source containing $1 \mathrm{mCi}$ was used. The model parameters were tuned up using a backpropagation algorithm with automatic regularization based on Bayesian interpolation [Mac92] (see section 2.5.2). This method is a supervised training algorithm where the input values and their corresponding desired output are presented to the network. To improve the learning and simulation procedures a simple rule based on prior knowledge has been used. This rule classifies directly the events separated by more than $1 \mu \mathrm{s}$ as non coincident. After training, we use the model to classify two single events as coincident or not coincident as follows: first, we calculate the inputs of the network from the data of each event (codification). Then, if these single detection events are separated by more than $1 \mu \mathrm{s}$, they are classified as non coincident. Otherwise, the two singles are considered a potential coincidence. The next step is to normalize the calculated input using information from the training set which is given to the ANN. Finally, a threshold is applied to the network output to decide whether the two singles are a coincidence or not. This process is represented in Fig. 54. 


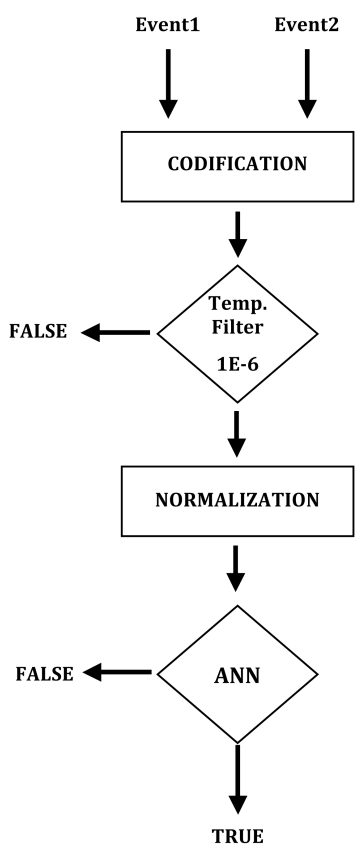

Figure 54: Schematic representation of the sorting method based on ANN.

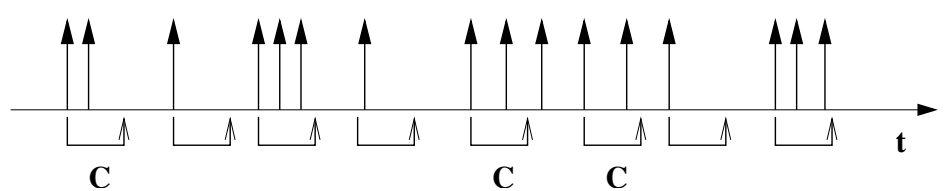

Figure 55: Schematic representation of the sorting method based on a fixed time window. Each single event is depicted as a vertical arrow according to its detection time. The horizontal bracket below the time axis represents the coincidence window. Events identified as coincidences are marked by a "C".

CONVENTIONAL SORTER We have also implemented a conventional coincidence sorting method which searches coincidences within the singles data stream using a fixed time window width, $\tau$. The first recorded single event opens a window; if the next event (and no other else) is found within that window, the pair is classified as a coincidence. If, on the contrary, no additional single is found, the event is considered a "single" and discarded. If more than one event are found within the same window, they are classified as a "multiple coincidence" and discarded as well. Additionally, a geometrical constraint is applied to remove those coincidences that imply a LOR that does not cross the FOV.

\subsubsection{Analysis}

When sorting single list mode data, it might occur that the two single photons assigned to a coincidence ("prompt") do arise from the same annihilation 
("true coincidence") or that they do not ("random coincidence"). On the other hand, it might also occur that the two photons created in the same positron annihilation are detected, and thus present in the single list mode data, but the coincidence sorter is unable to identify them as a pair. Thus, a potential coincidence is lost. From now on, we will refer to all the detected photon pairs present in the data as "real coincidences", regardless of being properly identified or not by any sorting method.

Simulated data allow us to access to all this kind of information, which remains unknown for real acquisitions. This information was used to evaluate the performance of the two coincidence sorters by means of the following figures-of-merit (FOM):

Efficiency (E): Defined as the ratio of true and real coincidences. The ideal value $E=1$ would imply that all detected pairs of correlated singles are correctly identified as coincidences.

Random Fraction (RaF) : Defined as the ratio of random coincidences and the total number of coincidences generated by the sorting procedure (prompts).

Moreover, we have studied the relationship between $\mathrm{E}$ and $\mathrm{RaF}$ for both sorting methods, by varying the window width for the conventional sorter, and the threshold value for the ANN sorter.

\subsubsection{Reconstruction algorithm and image analysis}

To assess the effect of the two sorters presented in this work from an imaging perspective, the resulting data has been reconstructed using an List-Mode Maximum-Likelihood Expectation-Maximization (ML-EM) implementation that runs in parallel on a multicore architecture [KWS $\left.{ }^{+} 09\right]$.

The system matrix has been obtained using a 3D analytical method [Ped09], based on an analytical model of the detector response function (DRF) $\left[\mathrm{SSD}^{+} 03\right]$. This method has been demonstrated to achieve high accuracy of the physical processes modeling compared to Monte Carlo methods, with the additional advantages that the analytical method is very fast and noise-free.

To evaluate the reconstructed image quality we have used the following FOMs:

Contrast-to-noise ratio (CNR):

$$
\mathrm{CNR}=\frac{\mu_{\mathrm{ROI}}-\mu_{\mathrm{bkgr}}}{\sigma_{\mathrm{bkgr}}}
$$

Relative standard deviation (RSD):

$$
\mathrm{RSD}=\frac{\sigma_{\mathrm{ROI}}}{\mu_{\mathrm{ROI}}}
$$

Contrast (C):

$$
\mathrm{C}=\frac{\mu_{\mathrm{ROI}}-\mu_{\mathrm{bkgr}}}{\mu_{\mathrm{bkgr}}}
$$


where the subindex ROI refers to the different regions of interest defined within the phantom, which were defined within the cold, the hot, and the warm background regions; $\mu$ and $\sigma$ are the mean and standard deviation, within the ROI. The values of CNR, RSD and $\mathrm{C}$ were obtained at various iterations.

\subsection{RESULTS \& DISCUSSION}

Fig. 56 show the graphs displaying RaF versus $\mathrm{E}$ for the point-like, line, mouselike and rat-like phantoms. It can be seen that the efficiency of the ANN method increases monotonically, and presents an asymptotic behaviour for $E=1$ (which would correspond to not applying any threshold). In contrast, the conventional sorter reaches a maximum efficiency for a certain window width. Interestingly, the optimal width for the time window depends on the object under study, and it does not necessarily coincides with the conventional width, ie. twice the Full Width at Half Maximum (FWHM) of the time resolution, as it is usually assumed. For larger windows, the efficiency of the conventional methods decreases, probably due to pile-up effects, whereas RaF increases.

Remarkably, for all the studied cases the ANN sorter provides less noisy results for a given efficiency.

This fact is also shown in Table 13, where RaF values are presented for a fixed value of $\mathrm{E}$, chosen to be the one obtained by the conventional sorter and $\tau=20 \mathrm{~ns}$.

The reduction of random coincidences in the data sample using ANNs is more significant for high activities; in those cases, the ANN-based sorted data still contained a large fraction of randoms. This effect is visible as an uniform background outside the phantom in the reconstructed image of the cylindrical phantom (see Fig. 57, right). However, the ANN-sorter was able to remove some artifacts (halos) which were present in the reconstructed images after applying the conventional sorter (Fig. 57, left). The cold rod was not properly reconstructed for any of the methods; this is probably due to the lack of statistics of the simulated phantom, and therefore a larger simulation will be needed to obtain enough coincidences to properly reconstruct the phantom image. For a mouse-like phantom and $0.2 \mathrm{mCi}$, the ANN-images showed a slightly better contrast and noise properties, and the resolution achieved for the reconstructed line-source was also better.

Figure 58 shows the relative improvement (in \%) for the ANN-sorter images over the conventional-sorter ones, as a function of the number of iterations, of contrast, RSD and CNR in four different ROIs: warm background (top left,A), hot rod (top right,B), image background (bottom left,C), and cold rod (bottom right,D), for the cylindrical phantom $(1 \mathrm{mCi})$.

The first thing to be noted is that there is always an improvement in the ANN results when compared with those of the conventional method for all the investigated FOMs (CNR, Contrast and RSD). 
The enhancements in the values of the FOMs are particularly marked for those ROIs with a low activity concentration. These ROIs are more sensitive to degradation effects derived from the presence of randoms in the sorted data. In the images of Fig. 57, it can be seen that these regions are slightly better reconstructed when ANN sorted data is used, especially for the image background and the warm background. Again, images with higher statistics should be investigated to further clarify this issue.

Table 13: Values of Random Fraction (RaF) for equal Efficiency (E).

\begin{tabular}{lccc}
\hline Source & E (20ns) & RaF(Conventional) & RaF (ANN) \\
\hline Line-off 0.2mCi & 0.98 & 0.27 & 0.20 \\
Line-off 1mCi & 0.92 & 0.65 & 0.48 \\
Line-off 5mCi & 0.68 & 0.90 & 0.76 \\
Mouse 0.2mCi & 0.99 & 0.32 & 0.23 \\
Mouse 1mCi & 0.94 & 0.70 & 0.56 \\
Mouse 5mCi & 0.75 & 0.92 & 0.81 \\
Rat 0.2mCi & 0.99 & 0.33 & 0.28 \\
Rat 1mCi & 0.95 & 0.71 & 0.59 \\
Rat 5mCi & 0.78 & 0.93 & 0.84 \\
Point 0.2mCi & 0.99 & 0.04 & 0.04 \\
Point 1mCi & 0.94 & 0.18 & 0.13 \\
Point 5mCi & 0.74 & 0.53 & 0.36 \\
\hline
\end{tabular}

\subsection{CONCLUSION \& FUTURE WORK}

In this work an application of ANN methods to the problem of reducing random coincidences has been investigated. It has been compared against a traditional coincidence sorting algorithm based on a time coincidence window and the application of geometrical conditions. When compared at the same efficiency, the ANN based method presents always a sorted output with a smaller RaF. However, under some circumstances the RaF can be too large for quantification purposes. It is still open how an estimation of the remaining randoms could be done for correcting the data.

In addition, the parametric curves linking $\mathrm{E}$ and RaF show different behaviors; while the conventional method presents a maximum achievable efficiency, the ANN-based one is able to increase the efficiency up to unity, the ideal value. In general, this comes at the price of a high RaF increase, although this depends on the geometry of the source. For point-like geometries it is possible to increase the efficiency while keeping $\mathrm{RaF}$ at an acceptable value.

The study based on reconstructed images was not conclusive, due to the low statistics of the simulated data. Also the large scanner gaps might be responsible of the poor quality of the images. Therefore, a more profound study is still necessary. 

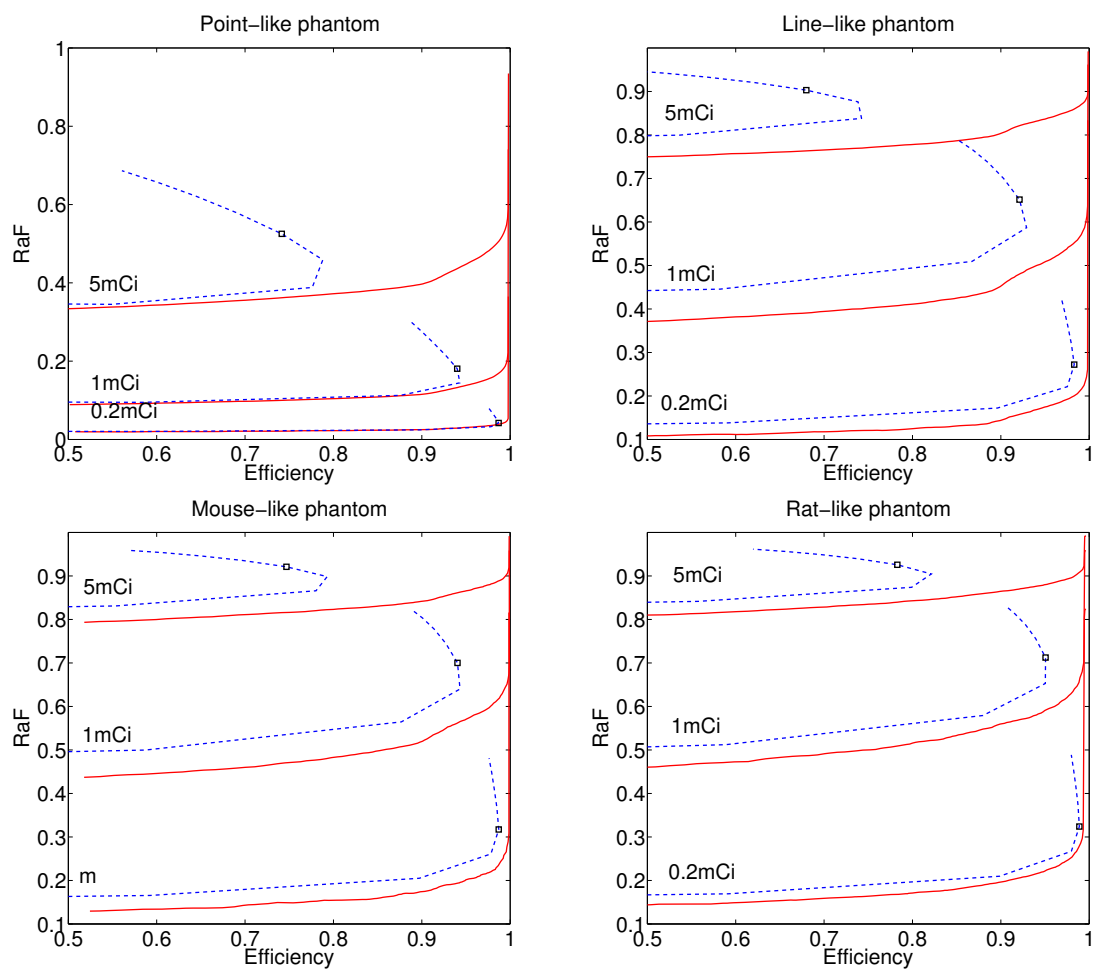

--- Conv. sorter
- ANN sorter

Figure 56: RaF vs E performance of the Conventional Sorting method (blue) for different window widths and the ANN sorting method (red) for various thresholds on the Point-like, Line-like, Mouse-like and Ratlike phantoms. The square, $\square$, indicates the values corresponding to the standard time coincidence window of $20 \mathrm{~ns}$.

Pattern recognition capabilities of ANN could fit better in more complex situations, where simple techniques such as the conventional sorter fails to model the underlying physics, for example, true multiple coincidences due to inter-crystal scatter, unconventional PET radioisotopes with undesired extra gamma emissions, etc. 
IDEAL
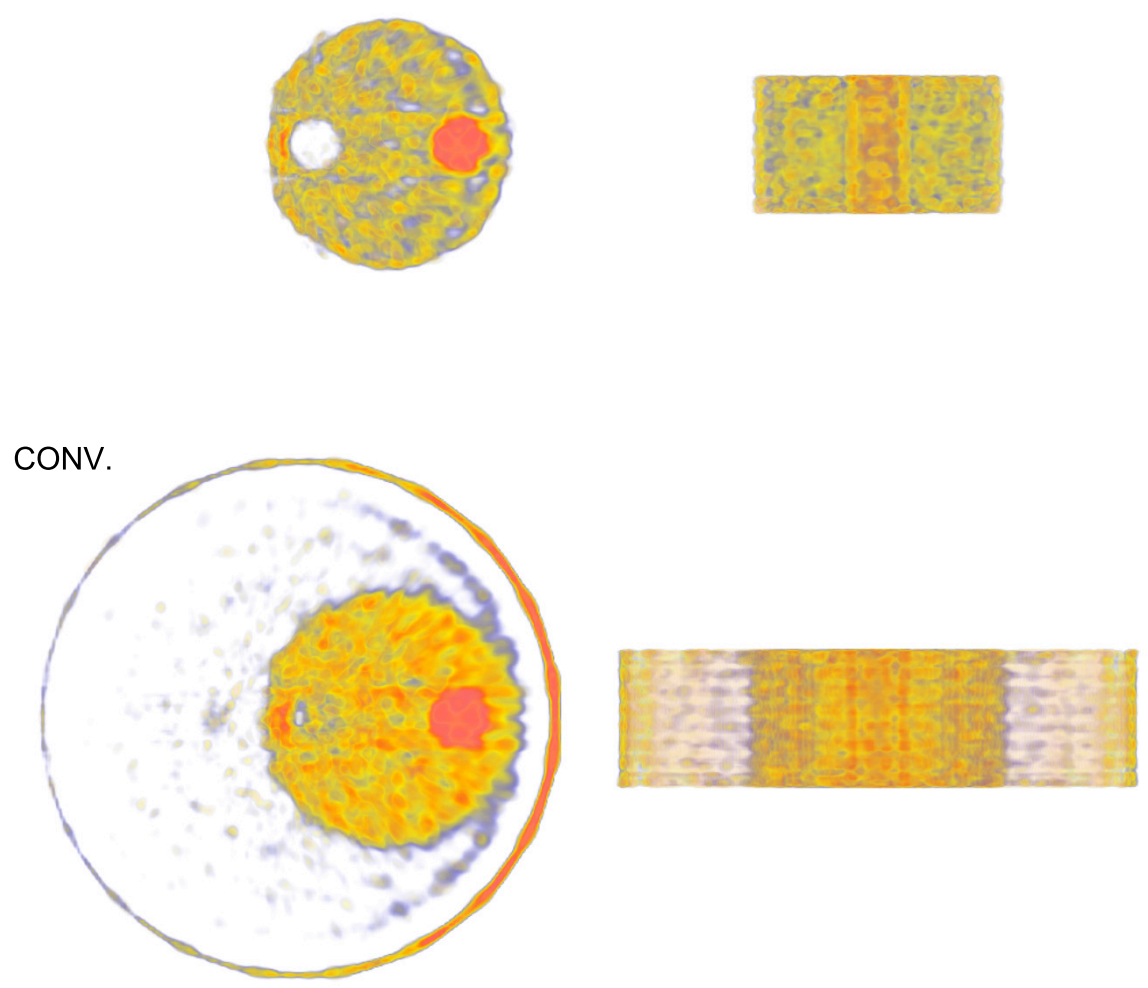

ANN
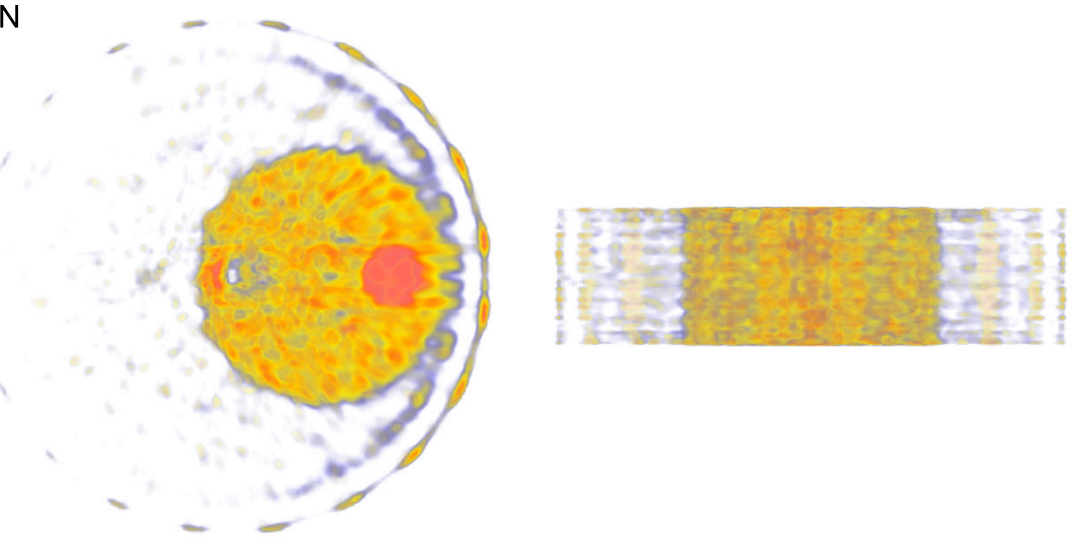

Figure 57: Reconstructed axial and coronal images for $1 \mathrm{mCi}$ cylindrical phantom using an ideal sorter containing all true coincidences (top), the conventional sorter (middle), and the ANN sorter (bottom). 

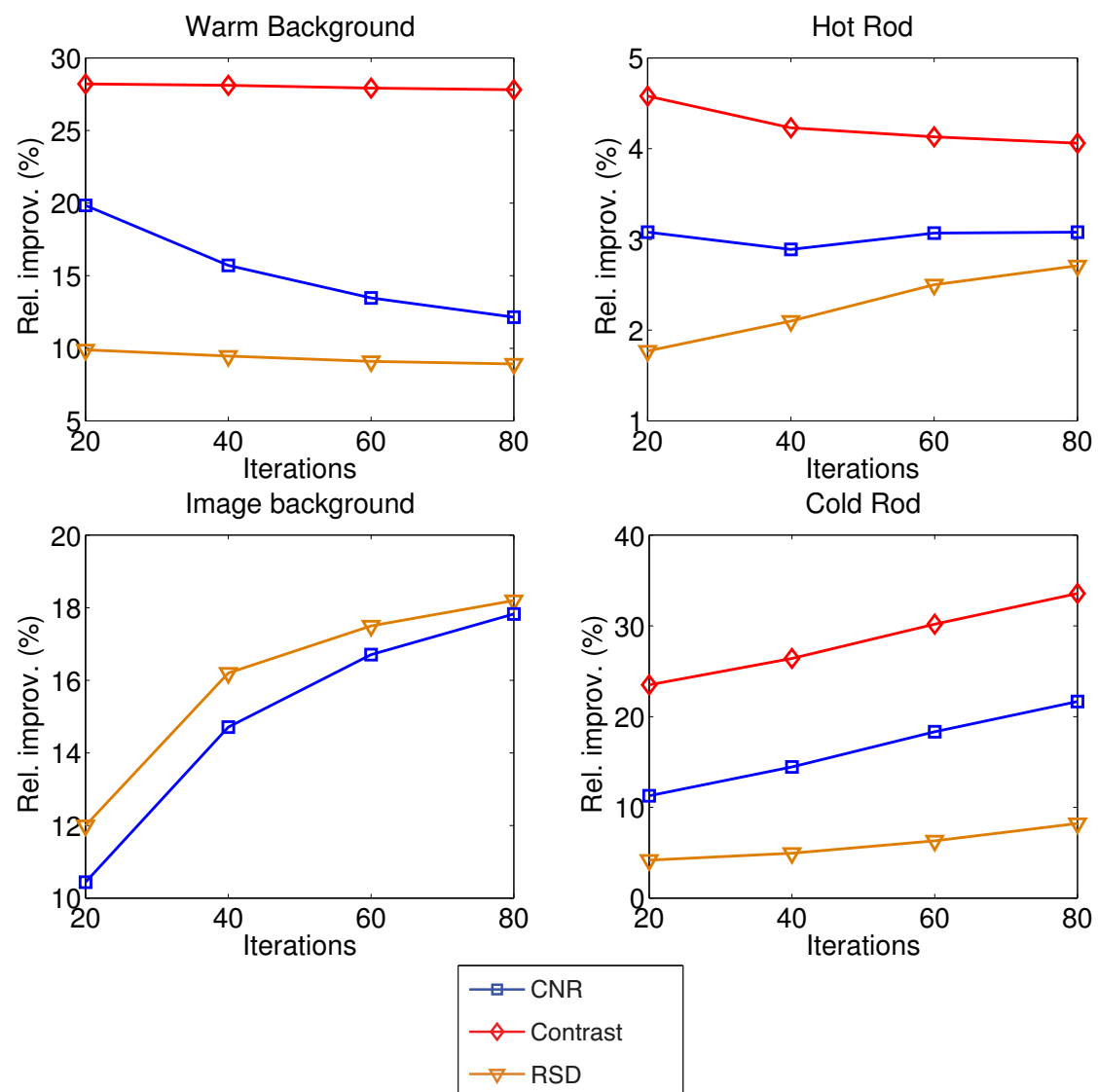

Figure 58: Relative improvement of RSD (in yellow triangles), Contrast (in red diamonds) and CNR (in blue squares) with the ANN-based method for each number of iterations in four different ROIs: warm background (topleft), hot rod (top-rigth), image background (bottom-left), and cold rod (bottom-rigth), for the cylindrical phantom $(1 \mathrm{mCi})$. 



\section{AUTOMATIC CLASSIFICATION OF ARRHYTHMIAS BASED ON ECG}

In this chapter, a contribution to the design and evaluation of supervised classification models based on multi-center datasets for arrhythmia detection and classification by ECG is presented. In this study, special attention is given to the evaluation of different feature selection methods and the effectiveness of the algorithms for quality control and alignment of pulses.

A part of this study, focused on the identification of significant points for the study of arrhythmias in electrocardiograms, has been presented in [FGAG $\left.{ }^{+} 11\right]$.

\subsection{INTRODUCTION}

The ECG has become a standard diagnosis test for the diagnosis of arrhythmias. ECG is widely used in clinical practice because it is affordable, not-invasive and effective. The ECG provides valuable information about the time taken by the impulse generated in the sinoatrial node to travel through the atria, the atrioventricular conduction system and the ventricles. Moreover it monitors the electrical activity of the heart through time and in different directional viewpoints.

Using the ECG, a trained cardiologist is able to classify each beat, and to detect abnormalities in the heart rhythm and ECG beat morphology. However, this detailed diagnosis is very time consuming and requires high expertise.

Different ML based systems to assist the cardiologist in the task of arrhythmia detection and classification have been developed in the past years. A proof of this is the number of published papers in this field [TKST08, PM10]. These studies includes among others, digital signal analysis[PFLM02], Fuzzy Logic methods [ZJ98], Artificial Neural Network [PFLM02, SM98] [LC89] [ZJ98], Hidden Markov Model [HPR04, MAA ${ }^{+}$05], Genetic Algorithm $\left[\mathrm{GPF}^{+}\right.$04], Support Vector Machines [OHM04], Self-Organizing Map $\left[\mathrm{LPB}^{+} 00\right]$, or Bayesian approaches [ $\left.\mathrm{LPN}^{+} 10\right]$. Although the effort done, there are not many annotated arrhythmia public databases, so most of nowadays publications are based on the MIT-BIH arrhythmia dataset generously compiled and published by the BIH Arrhythmia Laboratory between 1975 and 1979 $\left[\mathrm{MM} 01, \mathrm{MSM}^{+} 82\right]$.

The MIT-BIH database has some limitations that restrict the generalizability of the results obtained and their validation in a real clinical scenario. These 
limitations are due to: the use of only one center to compile the dataset, a low number of patients included (47 patients), and the use of only two leads (not coincident with the twelve currently used). The fact of not using multicenter datasets results in non-generalizable models, since it does not take into account the variations in the data when different scanners or procedures have been used, and the evaluations obtained may be optimistic. The low number of independent cases, does not allow the training of complex models, as well as lowers the robustness and generalization of the obtained model. Finally, the differences between the lead configuration used in MIT-BIH database and the one used in current acquisition protocols, do not make compatible the use of both models.

In this work a multi-center study consisting on 109 patients, has been done. It includes: (1) the design of a pre-processing and quality control pipeline for ECG data, (2) a new tool for manual ECG beat labeling for arrhythmia studies, (3) a beat alignment method, (4) a study of the number of variables needed to contain the most relevant information for the classification, and (5) an evaluation of the performance of a representative set of classifiers and feature extraction algorithms for classification of control cases and supra-ventricular arrhythmia versus ventricular arrhythmia.

\subsection{MATERiALS: THE SEE DATABASE}

The Sistema Experto en Electrocardiografía project (SEE) arrhythmia database has been compiled throughout the SEE project, and includes ECG exams provided by two clinical centers: Hospital de la Santa Creu i Sant Pau and Hospital Universitari Germans Trias i Pujol. The SEE arrhythmia database includes electrocardiographic records of 109 patients, consisting on 10 electrodes (RA, LA, RL, LL, $V_{1}, V_{2}, V_{3}, V_{4}, V_{5}, V_{6}$ ) and 12 lead classical configuration including: limb leads (I, II, III), augmented limb leads ( $\mathrm{VVR}, \mathrm{aVL}$, $\mathrm{aVF})$, and precordial leads $\left(\mathrm{V}_{1}, \mathrm{~V}_{2}, \mathrm{~V}_{3}, \mathrm{~V}_{4}, \mathrm{~V}_{5}, \mathrm{~V}_{6}\right)$.

Each record consists of approximately 10 seconds of ECG signal, obtained at $1000 \mathrm{~Hz}$ sample rate. The different diagnosis used in the SEE database to tag each beat have been defined using the recommendations of Prineas et. al. in [PCB82]. The number of patients per diagnosis and the number of pulses per diagnosis can be seen on Table 14 .

Table 14: Distribution of patients and beats per pathology.

\begin{tabular}{lcc}
\hline Label & Patients & Beats \\
\hline Supraventricular & 85 & 2897 \\
Ventricular & 33 & 380 \\
Normal & 56 & 947 \\
\hline
\end{tabular}


Concerning the pathologies considered in this study (supra-ventricular and ventricular) and the controls (normal beats), a total number of 4099 beats belonging to 109 different patients have been used. The ECG patterns Normal/Supraventricular beats (blue) and Ventricular beats (red), for the different leads used for classification (I,II, III, V1,V2,V3,V4,V5,V6) are presented in 59.

\subsection{METHODS}

To achieve the main objectives of this study we have designed a framework to process, label, and train the models. This framework includes an automatic pipeline to process the ECG data, an arrhythmia annotator software, a quality control and beat alignment module. Moreover a CV strategy has been implemented to train and evaluate each feature extraction and classification algorithms. The diagram of the framework implemented and the workflow of the data included in this work can be seen in Figure 60.

\subsubsection{Pre-processing, QRS detection, and beat labeling}

The supervised classification approach considered in this work requires that all beats are labeled with their associated diagnosis. This task requires a high amount of time and needs to be done by expert cardiologists. Moreover the ECG signal needs to be processed before using it for diagnosis and the quality of the ECG signal needs to be controlled. To do so, we have used the Arrhythlab software designed and implemented during the SEE project, and described in section 3.3.

\subsubsection{Quality Control}

In order to ensure the quality of the ECG signals used in Arrhythlab, a quality control module has been developed. This module is designed to exclude from the database the noisy ECG, and the ECG studies that contain some electrodes disconnected or with very low signal intensity due to incorrectly connection.

Two parameters have been defined to characterize the quality of each ECG: The SNR and $\tau$.

The SNR ratio has been obtained from the estimations of signal and noise contribution defined as:

NOISE

$$
\text { noise } i, l=\frac{\sum_{t=0}^{0.1}\left|B_{i, l}(t)-\widetilde{B_{i, l}(t)}\right|+\sum_{t=0.3}^{0.4}\left|B_{i, l}(t)-\widetilde{B_{i, l}(t)}\right|}{n}
$$



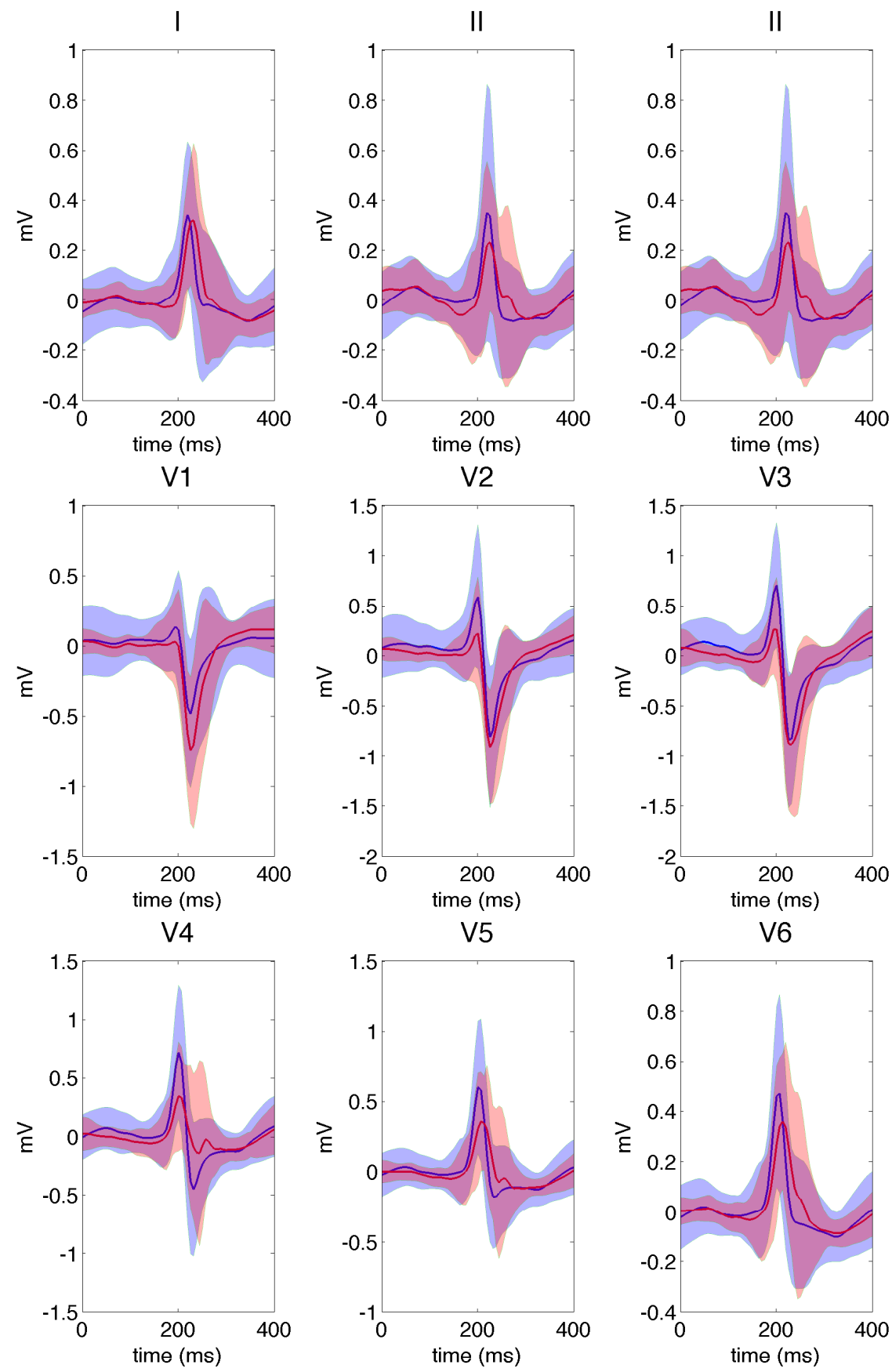

Figure 59: ECG patterns of Normal/Supraventricular beats (blue) and Ventricular beats (red), for the different leads used for classification (I,II,III, VI,V2, V3, V4, V5, V6). The Mean ECG beat of each class is shown as a solid line and its standard deviation in shadowed colour. The purple region corresponds to the region of coincidence among the both patterns. 


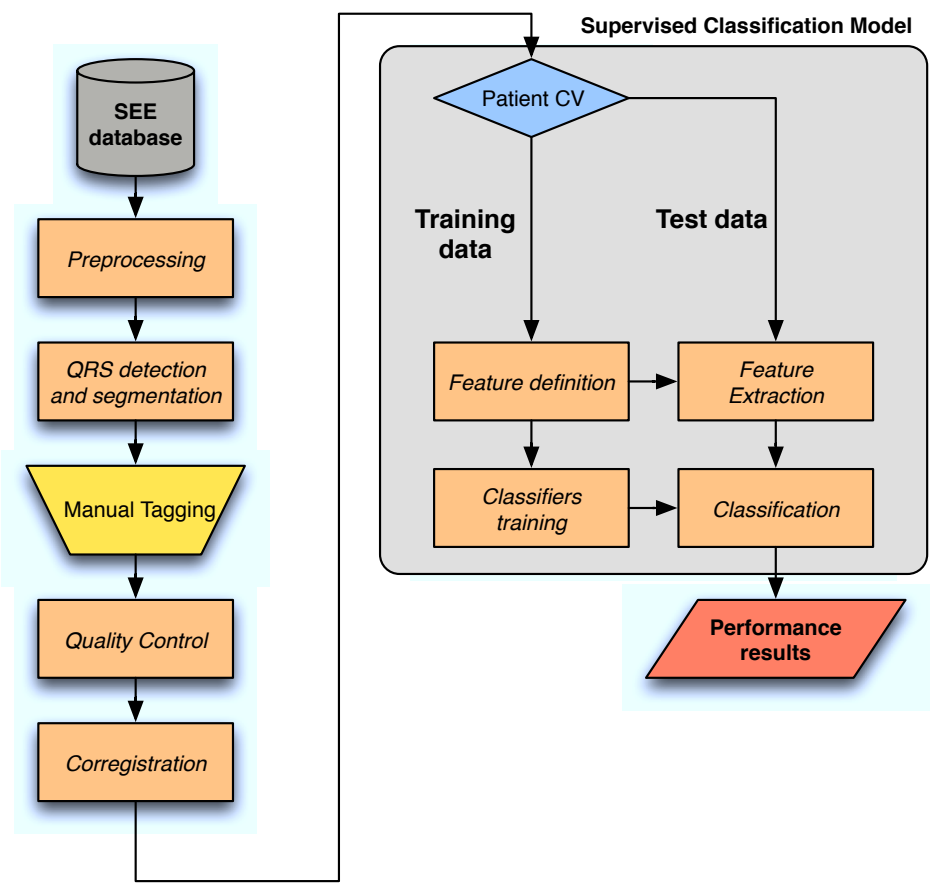

Figure 60: Workflow diagram

where $i$ identify each beat, and $l=1, . ., 12$ each lead, $\widetilde{B_{i, l}}$ is a smoothed version of $B_{i, l}$, and $n$ is equal to the number of samples in the intervals $[0,0.1]$ and $[0.3,0.4]$. Taking into account that the sample rate of the ECG records is $1000 \mathrm{~Hz}$, then $\mathrm{n}=400$.

SIGNAL

$$
\operatorname{signal}_{i, l}=\max \left|B_{i, l}([0.1,0.3])\right|
$$

and therefore the SNR for the beat $i$ and the lead $l$ is defined as:

$$
\mathrm{SNR}_{i, l}=10 * \log 10\left(\operatorname{signal}_{i, l}^{2} / \text { noise }_{i, l}^{2}\right)
$$

finally, the SNR assigned to the $i$ beat is defined as the minimum SNR obtained in their different leads:

$$
S N R_{i}=\min _{l}\left(S N R_{i, l}\right), l=1,2 \ldots 12
$$

The parameter $\tau$ is defined to detect leads with no signal or very poor signal due to lead connection problems. We define $\tau$ as:

$$
\tau_{i, l}=\sum_{t=0}^{0.4}\left|B_{i, l}(t)-M_{l}(t)\right|
$$




$$
\tau_{i}=\min _{l}\left(\tau_{i, l}\right), l=1,2 \ldots 12
$$

where $M_{l}(t)$ is the mean beat obtained from the SEE database for lead $l$.

In Figure 61 we can see the distribution of the beats of the SEE database in function of SNR and $\tau$

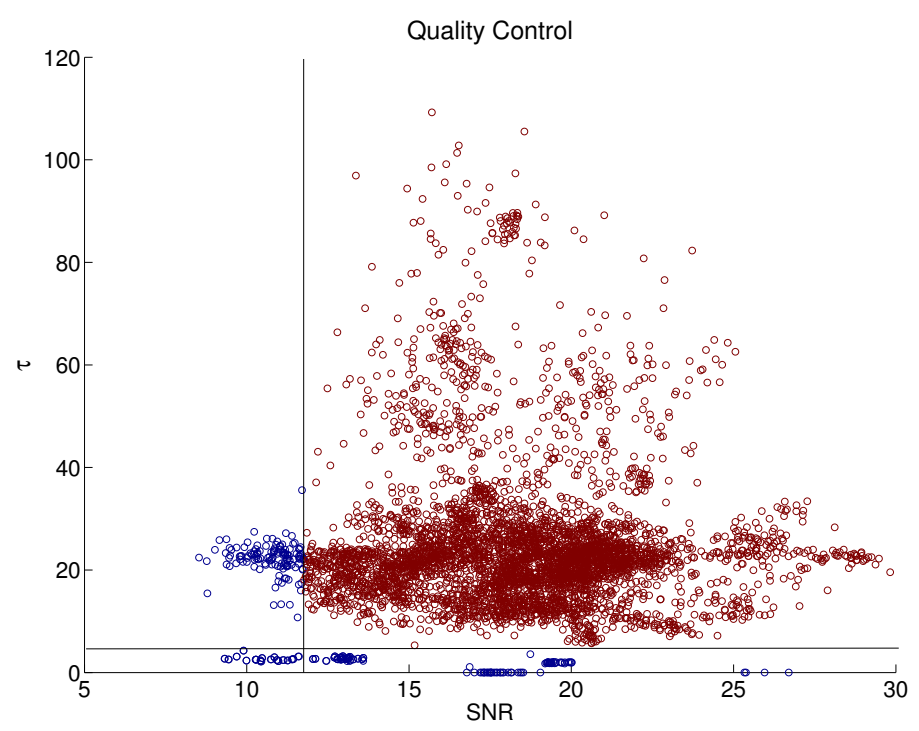

Figure 61: SNR vs $\tau$ scatterplot of the beats contained in the SEE database. The small red circles represent the beats in the dataset that have passed the quality control, and the blue ones represents the beats that have not passed the quality control.

Finally, the conditions for an ECG to be accepted for the analysis have been defined by a $S N R>11.78$ and a $\tau>5$

\subsubsection{Beat alignment}

With the aim of optimizing the feature extraction task and improving the performance and robustness of the classifiers, an alignment module has been developed. It is based on the estimation of a possible shift between the estimated mean beat and each beat sample. The estimation of the shift $\delta$ for each lead $l$ and beat $i$ is based on finding the maximum cross-correlation value $B_{i, l} \star \overline{B_{l}}$ between both signals.

$$
\begin{gathered}
\left(B_{i, l} \star \overline{B_{l}}\right)(t)=\sum_{s=-\infty}^{\infty} B_{i, l}(s) \overline{B_{l}}(t+s) \\
\delta_{i, l}=\arg \max _{t}\left(B_{i, l} \star \overline{B_{l}}\right)(t)
\end{gathered}
$$


where $\overline{B_{l}}$ is the mean beat estimated for lead $l$.

Although each lead $l$ has an associated $\delta_{i, l l}$ value for each beat, is necessary to apply the same beat shift to all the leads to ensure the alignment between the different leads used. To do so, a unique value $\delta_{i}$ is applied to all leads for each beat, where $\delta_{i}$ defined as follows.

$$
\delta_{i}=\arg \max _{l, t}\left(B_{i, l} \star \overline{B_{l}}\right)
$$

\subsubsection{Dimensionality reduction based on prior knowledge}

In order to reduce the data dimensionality based on the available knowledge about the original data, two transforms were applied on the data:

In a first step we performed a sub-sampling to the data, reducing to onesixth the number of points that describe the ECG. This sub-sampling does not have to affect significantly the information contained on the signal, because although the ECG are obtained with a sampling frequency of $1000 \mathrm{H} z$, a filter has been applied to remove $45 \mathrm{~Hz}$ noise in the ECG and the high-frequency detail.

In a second step we removed the leads I, II, and III because they can be obtained as linear combinations of aVL, aVF and aVR and only provide redundant information to the problem.

Finally, the number of points representing a pulse where reduced from 4801 to 601 .

\subsubsection{Feature extraction}

Although a dimensionality reduction based on prior knowledge has already been performed on the database, the use of FE algorithms could optimize the number of variables, simplifying the classification models and increasing the robustness of the predictions.

Several FE techniques have been included in this study. These are: Relief-F, PCA, ICA, WAV+PCA, and FC. A brief description of these algorithms, can be found in Section 2.5.1.

\subsubsection{Classifiers}

As it happens in the case of FE methods, several widely used classification methods have been included in the study. These are: LDA, QDA, KNN, and ANN. In the case of KNN, different $k$ values $k=[10,20]$, and different distance metrics - euclidean and city block (cb)- have been included in the 
study. Finally, in the case of ANNs four different architectures have been tested including ANNs with: a single hidden layer of 20 neurons ([20]), two hidden layers with 10 and 5 neurons in each layer respectively [10 5], two hidden layers with 10 and 10 neurons in each layer respectively [10 10], and finally two hidden layers with 20 and 10 neurons in each layer respectively [20 10]. The ANNs included in this study use a Levenberg-Marquardt backpropagation training function that updates weight and bias values according to LevenbergMarquardt optimization. A brief description of these algorithms can be found in Section 2.5.2.

A different number of variables $n$ have been considered as the input of the classification algorithms to evaluate their influence in the performance of the classifiers. Specifically $n=[28,37,46,65,81,101,121]$ variables have been considered for the analysis. The inputs of the classifiers were the $n$ most relevant features extracted directly from the ECG signal. Moreover, the R-R interval-time has been introduced as an additional input.

\subsubsection{Evaluation strategy}

An evaluation strategy based on K-fold CV with $\mathrm{K}=10$ (as suggested in [Koh95]) has been defined to evaluate the performance of each FE and classification method. Notice that the cross-validation has ben applied on the patients and not on the ECG beats because we have considered nonindependence between the beats of the same patient, and therefore we avoid the use of beats from the same patient to train and test at the same time. In order to evaluate the performance in the classification we have considered the G measure because of their suitability in case of unbalanced datasets.

\subsection{RESULTS}

In this study, an evaluation of the performance of a representative set of classifiers and FE algorithms for classification of control cases and supraventricular arrhythmia versus ventricular arrhythmia has been done. The results presented in this section also include the study of the number of variables needed to contain the most relevant information for the classification, and the differences on performance when using a beat alignment method before classifying.

\subsubsection{Patient dependence analysis}

Before proceeding with the evaluation of the classification models, the dependence of the ECG features with the patient to which it belongs has been analyzed. To do so, we have performed a PCA of the ECG points. Once the PCA scores are calculated, we have used the values of two first principal 
component to build a 2D scatter plot of every beat contained in the SEE dataset (see Figure 62). The Normal/supraventricular beats are marked as $\nabla$, the ventricular beats as $\circ$ and each color corresponds to a different patient. This dimensionality reduced representation allows us to see whether the ECG features are mostly dependent on their class tag or on the patient to which it belongs.

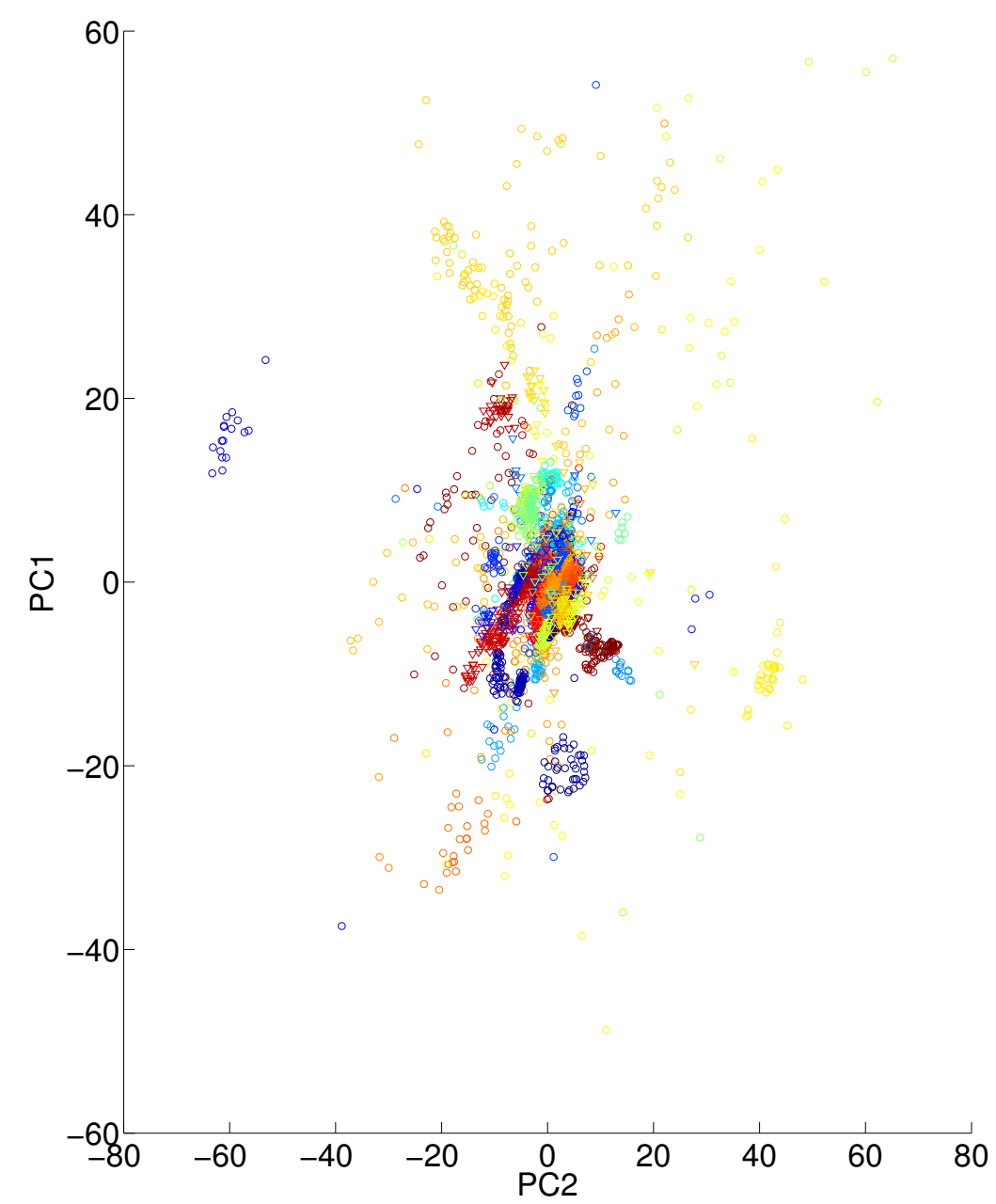

Figure 62: Scatter plot of the two first principal component coefficients of every beat contained in the SEE dataset. The Normal/supraventricular beats are marked as $\nabla$, and the ventricular beats as $\circ$. Each color corresponds to a different patient. 


\subsubsection{Number of variables}

In the design of a classification model, it is mandatory to select an optimal number of variables that describe the input data correctly without increasing significantly the model complexity. In this sense, we performed a study of the influence of the number of the input variables in the classification performance.

To do so, different classifiers were trained and tested using as a input the $\mathrm{n}$ variables (or features) with higher scores according to the FE method used. The experiment was repeated for different values of $n$ including $\mathrm{n}=$ $[28,37,46,65])$.

In Figure 63, the dependence of the mean performance on the number of input variables used is presented. These performance values were calculated using the results obtained for all classification algorithms included in the study.

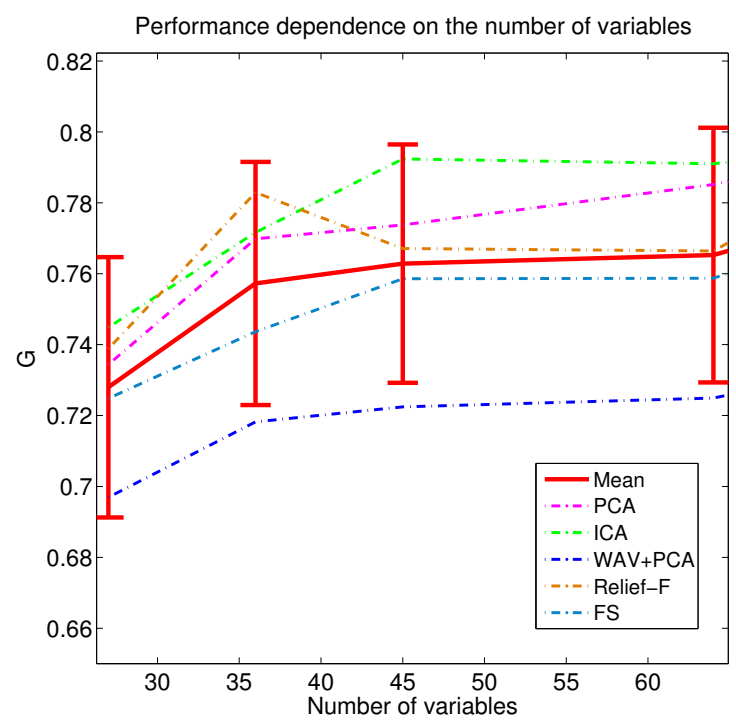

Figure 63: Performance vs. number of variables used by the classifiers. The performance values were calculated using the mean performance obtained for all classifiers and FE methods.

\subsubsection{Effect of alignment}

In order to analyze the influence of the alignment algorithm proposed in the classification performance, we used all the classification models included in this study (with $n=64$ ) to compare their performance in both scenarios, when they are trained and tested with aligned ECG signals and with non aligned ECG signals. The results are presented in a box plot in Figure 64. 


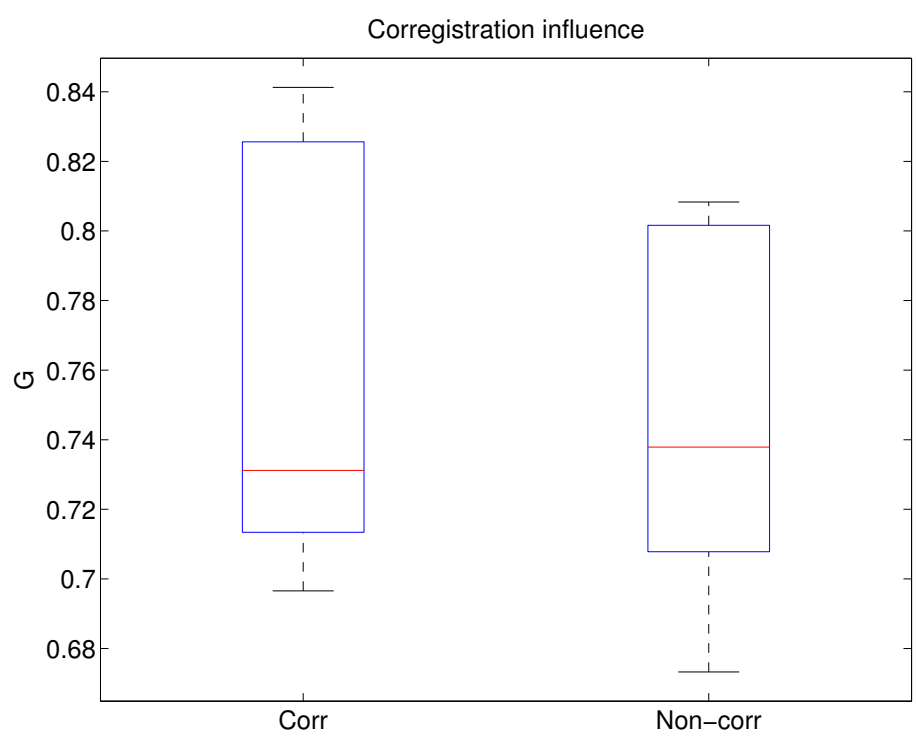

Figure 64: Performances obtained with and without alignment. The performance values of this box-plot has been obtained using all the FE methods considering 64 variables, and all the classifiers included in the study.

\subsubsection{Feature extraction and classification algorithms}

A comparison between the classification performances obtained when using the different FE methods was performed. The results obtained are presented in Figure 65 . The classification performances were obtained as the mean of the performances obtained using each of the classification algorithms included in the study. The experiment was done using the first 64 features $(n=64)$ extracted by each FE algorithm from the aligned SEE dataset.

In analogy with the study of the FE methods, a comparison between the classification performances obtained when using the different classification methods was performed. This comparison can bee seen in Figure 66. The classification performances were obtained as the mean of the performances obtained using each of the FE algorithms included in the study. The experiment was done using the first 64 features $(n=64)$ extracted by each FE algorithm from the aligned SEE dataset.

Finally, the classification performances (G) for each classification algorithm and FE method is presented in detail in Table 15.

\subsection{DISCUSSION}

In this work a study of the performance of the mostly used ML based methods for the automatic classification of arrhythmia has been done. 


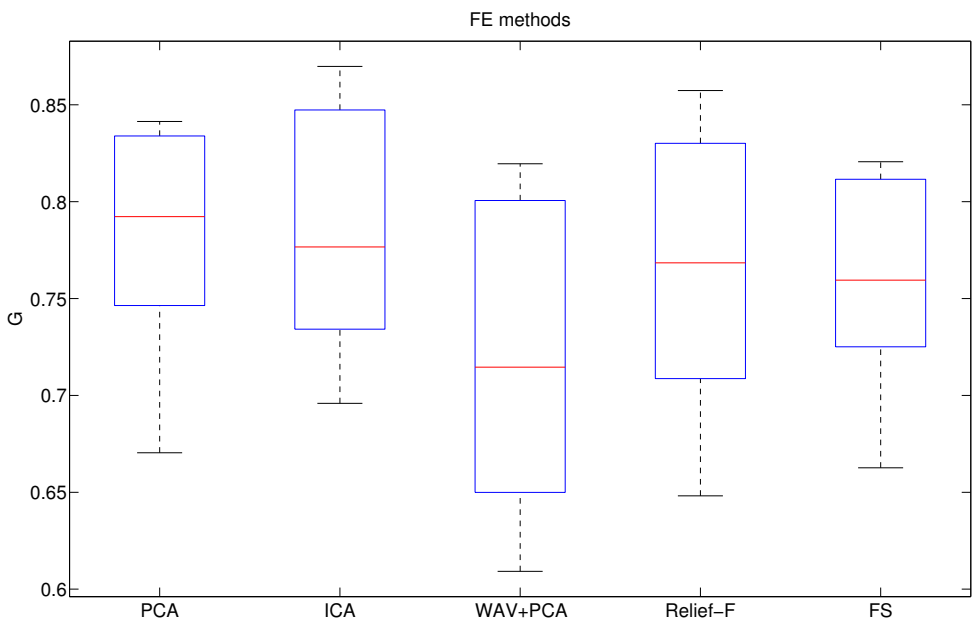

Figure 65: Mean performances obtained using different FE methods.

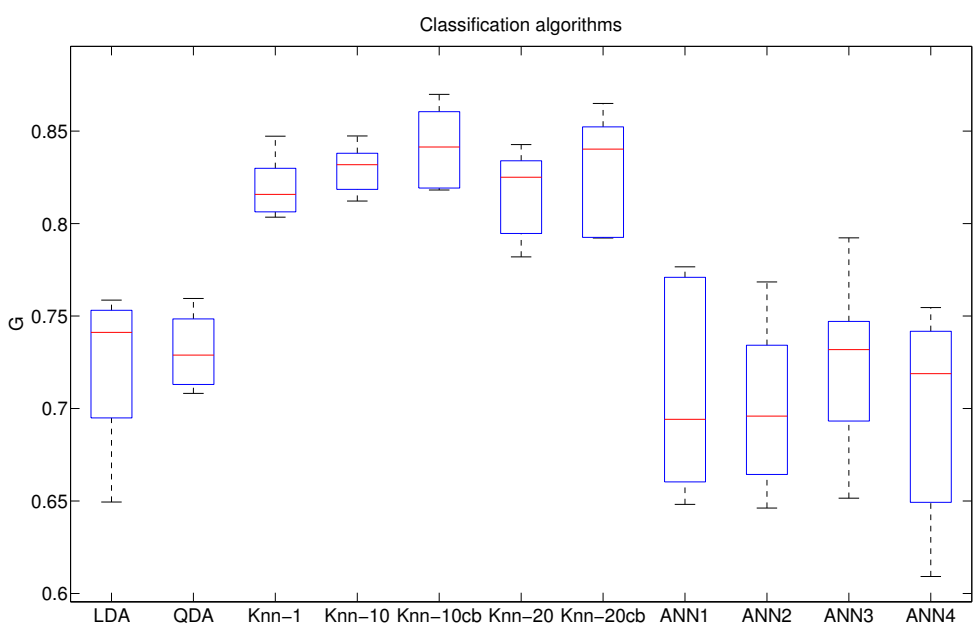

Figure 66: Mean performances obtained using different classification algorithms. 
Table 15: Performance obtained for each combination of FE and classification algorithm in terms of $\mathrm{G}$ values. The results were obtained using the first 64 features $(n=64)$ extracted by each FE algorithm from the aligned SEE dataset.

\begin{tabular}{cccccc|c}
\hline & PCA & ICA & WAV+PCA & Relief-F & FC & MEAN \\
\hline LDA & 0,75 & 0,74 & 0,65 & 0,71 & 0,76 & 0,72 \\
QDA & 0,74 & 0,73 & 0,71 & 0,71 & 0,76 & 0,73 \\
KNN-1 & 0,82 & 0,85 & 0,80 & 0,81 & 0,82 & 0,82 \\
KNN-10 & 0,83 & 0,85 & 0,81 & 0,83 & 0,82 & 0,83 \\
KNN-10 cb & 0,84 & 0,87 & 0,82 & 0,86 & 0,82 & 0,84 \\
KNN-20 & 0,83 & 0,84 & 0,78 & 0,83 & 0,80 & 0,82 \\
KNN-20 cb & 0,84 & 0,86 & 0,79 & 0,85 & 0,79 & 0,83 \\
ANN1 & 0,77 & 0,78 & 0,69 & 0,65 & 0,66 & 0,71 \\
ANN2 & 0,67 & 0,70 & 0,65 & 0,77 & 0,72 & 0,70 \\
ANN3 & 0,79 & 0,73 & 0,65 & 0,71 & 0,73 & 0,72 \\
ANN4 & 0,74 & 0,75 & 0,61 & 0,72 & 0,66 & 0,69 \\
\hline MEAN & 0,78 & 0,79 & 0,72 & 0,76 & 0,76 & 0,76 \\
\hline
\end{tabular}

Unlike most studies published to date, this one uses a multi-center dataset (SEE dataset). SEE triples in number the cases included in most used public database for arrhythmia studies, the MIT - BIHdatabase. Moreover, the lead configuration used by SEE dataset (consisting on 12-leads), is the most widespread in these studies, unlike the configuration of only two leads used by the MIT - BIHdatabase. For this study it was necessary to design a beat labeler software that would allow the collection and annotation of patient's pulses by expert cardiologists.

This study does not only present the results of different combinations of feature extraction methods and classification algorithms it also addresses, the automatic quality control of these signals and the alignment correction of the segmented beats.

The quality control method designed allows efficient detection of low signalto-noise ratio ECGs, or those ECG with low signal problems in any of its derivations. $6 \%$ of the beats recorded have been eliminated due to not satisfy the quality control.

On Figure 62 it is shown how the beats belonging to the same patient (same color) are not uniformly distributed in the feature space, but clustered. This demonstrates a strong dependence of the ECG with the patient which they belong to. This dependence has been taken into account in the design of the evaluation strategy, by avoiding the use of beats belonging to the same patient to both, train and test. 
It is shown in Figure 64 how the application of the aligning algorithm slightly improves (although not significant) the results obtained with the absence of alignment. It is important to notice that for this calculation were taken into account all FE methods and classifiers included in this study.

Regarding the analysis of the optimal number of variables to represent the information contained in the ECG, different tendencies were observed depending on the type of feature extraction method used (see 63). In general terms ICA gets the best performance, reaching the best performances in terms of $\mathrm{G}$ from $\mathrm{n}=46$ variables. Relief-F also obtains a good performance with few variables, however when the number of variables $n$ were higher that 37 , its performance decreased probably due to the introduction of redundant information.

The results obtained using different FE methods (see Figure 65) were calculated taking into account the results obtained with all the classification algorithms included in the study. These results do not show significant differences in terms of $\mathrm{G}$ between the different FE methods. Although these small differences between methods, it can be seen that ICA obtains the highest values of $\mathrm{G}$.

Analysis of the different classification algorithms reveals that the use of KNN for the classification of this kind of signals is the most effective method. The results obtained with KNN when using a cb distance rather than Euclidean, improves the performance in the classification. Furthermore, it is observed that a number of neighbors $k=10$ close to the square root of the number of variables $\sqrt{64}$, is optimum for the classification with KNN. The results show that dependence of the ECG with the patient to which they belong, require of complex and disjointed decision boundaries that cannot be approximated with simple linear or quadratic boundaries. Finally, ANN based classifiers show high variability in the performance values obtained due to their dependence on the initialization values used.

Although this is a multi-center study in future work it should be extended including more centers geographically distant in order to increase the reliability of the results obtained.

\subsection{CONCLUSION}

To conclude, this study has included the following contributions: implementation of a pipeline for ECG signal pre-processing; design of a quality control module, a study of the optimum variables needed for the analysis, and it also presents a study on the adequacy of different classification methods and feature extraction methods for the automatic analysis of ECG signals. 
Part III

CONCLUSIONS 



\subsection{CONCLUSIONS}

Taking into account the proposed objectives and the results achieved in this $\mathrm{Ph} . \mathrm{D}$. Thesis, the following conclusions have been obtained:

- CO1 - An open source toolbox for MRSI processing and visualization allow quick development techniques for analysis and processing of MRSI, and simplify the design of multi-center studies involving MRSI datasets obtained by NMR scanners from different manufacturers. The design and implementation of the MRSILab open source toolbox for automatic processing and visualization of MRSI data has contributed in various European institutions and clinical centers research.

- CO2 - The implementation of ArrythLab has facilitated the compilation of annotated ECG datasets. Throughout the project Sistema Experto en Electrocardiografía, 108 ECG records have been compiled and annotated using ArrythLab, saving much time of highly qualified personnel.

- CO3 - A semi-automatic processing pipeline for ${ }^{1} \mathrm{H}$-MRS signals has been integrated in CURIAM-BT. This processing pipeline ensures the reliability and repeatability of the results obtained by the integrated predictive models. The current version of CURIAM- BT that includes the pipeline described in this work is already being evaluated at Hospital Universitario Doctor Peset, at the Clínica Quirón and Hospital de la Ribera, to be deployed.

- CO4 - The compatibility study of existing classifiers based on 1.5T datasets when they are used on $3 \mathrm{~T}^{1} \mathrm{H}$ SV-MRS, suggest that existing classifiers based on 1.5T datasets are applicable to classification of 3T ${ }^{1} \mathrm{H}$ SV-MRS data. Since the methods used in this study are available on existing software [ $\mathrm{SGGV}^{+} 08, \mathrm{PROCJS}^{+}$08], the conclusions obtained have immediate implications for the use of the currently-available multi-centre brain tumour datasets and prediction models that are based on 1.5T MR spectra.

- CO5 - A novel FE method, RSFDA, has been presented for the classification of brain tumor MRS spectra. The application of RSFDA constitutes an interesting alternative to other FE methods and also complements other techniques in datasets with high baseline deformations. Moreover, RSFDA provides a spectrum reconstruction where the most discriminant 
characteristics are emphasized, and, therefore, allows an easy interpretation of the results. The functional representation of the spectra opens up the possibility of using the alignment techniques described by Ramsay et. al. to improve robustness against frequency shifts or to perform analysis based on spectra derivatives. Finally, RSFDA has exhibited robustness against the most common artifacts in MRS. It has also demonstrated better as well as a higher performance than FC and results similar to PCA when using the same number of variables.

- CO6 - The design of an ANN based model for the problem of reducing random coincidences in small animal PET have improved the performance of traditional coincidence sorting algorithm based on a time coincidence window and the application of geometrical conditions. Comparing both methods at the same efficiency values, the ANN based method always presents a sorted output with a smaller random fraction. Moreover, in contrast to sorting methods including geometrical conditions, our ANN based model does not present a maximum achievable efficiency limit. Pattern recognition capabilities of ANN could fit better in more complex situations, where simple techniques such as the conventional sorter fails to model the underlying physics (e.g. true multiple coincidences due to intercrystal scatter, unconventional PET radioisotopes with undesired extra gamma emissions).

- CO7 - The multi-center evaluation of automated arrhythmia detection and classification models, suggest that the quality control method designed allows efficient detection of low signal-to-noise ratio ECG, or those ECG with low signal problems in any of its derivations. Furthermore, the aligning algorithm designed and implemented in this work slightly improves the results obtained with the absence of alignment. In general terms, ICA gets the best performance, in terms of number of variables needed, and in term of $G$ in the classification problem. Analysis of the different classification algorithms reveals that the use of KNN for the classification of this kind of signals is more effective than more complex algorithms such as the ANN

\subsection{FUTURE WORK}

Some of the future lines of investigation directly related to the results of this $\mathrm{PhD}$ Thesis are:

- FW1 - Development of a MRSIlab module to allow the inclusion of tissue classification models based on MRSI-MRI data.

- FW2 - Development of brain tumour classifiers based on MRS using datasets that include mixed samples from 1.5T and 3T NMR scanners. 
- FW3 - Generalize the applicability of RSFDA to different classification problems that involve the use of signals.

- FW4 - PET simulations with higher statistics will be run to perform a complete evaluation of images reconstructed from coincidences sorted using the presented ANN based algorithm.

- FW5 - Extending the study of automatic classification of arrhythmias based on ECGs, including clinical data concerning the patient, and new samples collected from different clinical centers. Moreover, study the application of ML algorithms that can take profit from new non-labeled ECG samples .

- FW6 - Study non-linear manifold embedding techniques for the extraction and visualization of biomedical signals features.

- FW7 - Study non-i.i.d. methods with sparcity properties (e.g Convex Non-Negative Matrix Factorization) to characterize biomedical signals for classification 

Part IV

APPENDIX 



\section{A.1 THE B-SPLINES BASIS}

In order to define a spline we need to divide the interval over which the signal is to be approximated into $m$ subintervals, defined by the values $k_{1} \leqslant k_{2} \leqslant$ $\cdots \leqslant k_{\mathrm{m}}$ called knots or breakpoints. In the B-spline representation, we can adjust the flexibility in the signal fitting increasing the number of knots in the regions where the signal exhibits the most complex variation, and decreasing the number of knots in the less complex ones. When the knots are equidistant the B-spline is said to be uniform, otherwise non-uniform. In Figure 67 an example of uniform and non-uniform B-spline basis and their fitting results are presented.

When the positions of the knots are defined, we can approximate a signal $y(t)$ with a linear combination of basis B-splines $\phi_{i, n}$ of degree $n$

$$
y(t) \simeq x(t)=\sum_{i=1}^{m-n-1} c_{i} \phi_{i, n}(t)
$$

where $c_{i}$ are the $m-n-1$ coefficients that, given a particular basis B-splines, describe the signal $y(t)$.

The $m-n-1$ B-splines basis functions of degree $n$ can be computed, for $n=1,2, \ldots, m-1$, using the Cox-de Boor recursion formula,

$$
\begin{gathered}
\phi_{i, 1}(t)= \begin{cases}1, & \text { if } k_{i} \leqslant t \leqslant k_{i+1} \\
0 & \text { otherwise }\end{cases} \\
\phi_{i, n}(t)=\frac{t-k_{i}}{k_{i+n}-k_{i}} \phi_{i, n-1}(t)+\frac{k_{i+n+1}-t}{k_{i+n+1}-k_{i+1}} \phi_{i+1, n-1}(t),
\end{gathered}
$$

where $i=1, \ldots, m-n-1$.

As can be seen in Figure 68, an order n B-spline basis function is positive only over $n$ adjacent intervals. Therefore B-slines basis are compact support functions. This behavior is of a great relevance for efficient computation.

Notice that the variables needed for fitting $y(t)$ (or degrees of freedom) are equal to the order of the $\mathrm{B}$-spline degree $n$ plus the number of interior knots $m$ less 1 , that is $n+m-1$. 

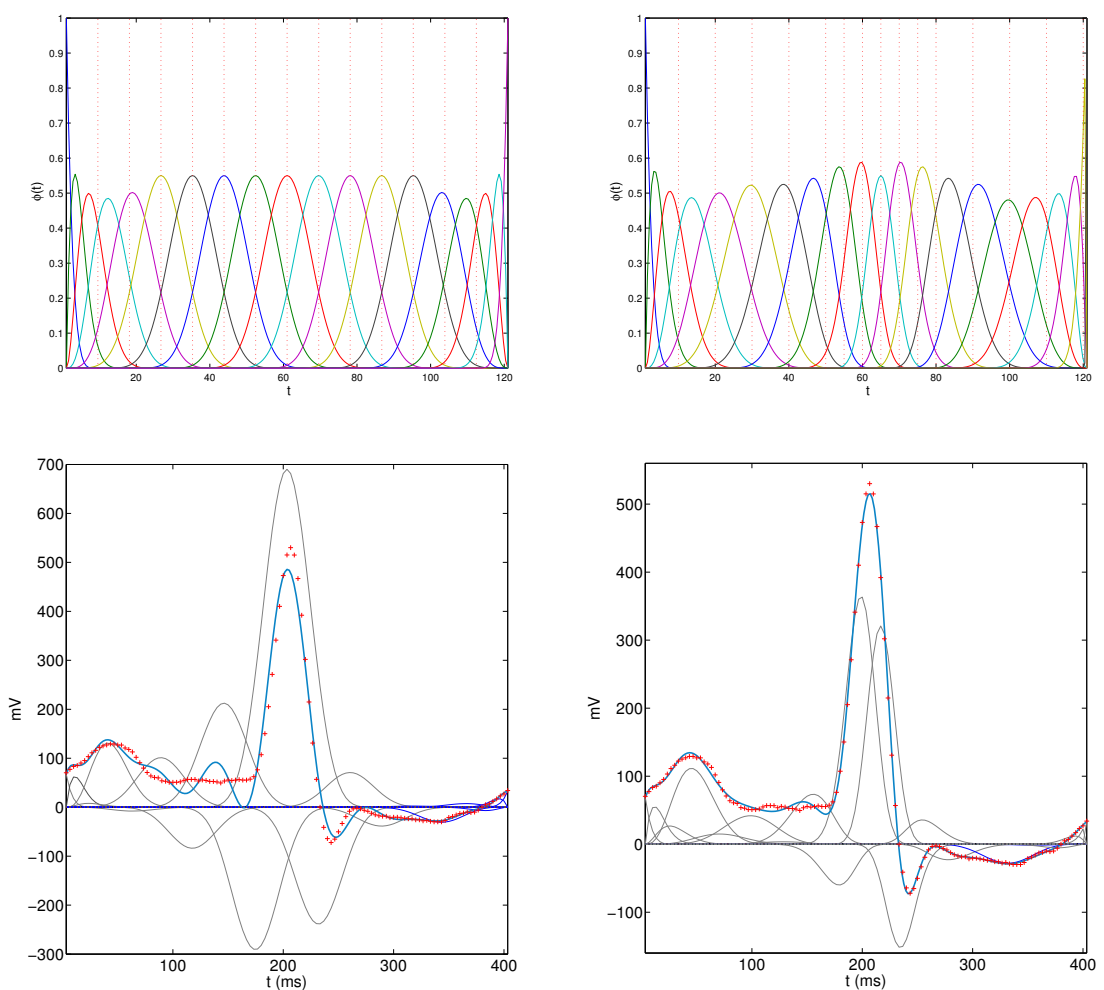

Figure 67: B-splines fitting of a real ECG using uniformly spaced knots (left) and nonuniformly spaced knots (right). The plots on top present the B-spines basis functions used for the fitting. The plots on the bottom present the results of the fits. In these last plots the fitting function obtained is presented in blue line, the original ECG signal samples used for the fitting are presented in red crosses, and the B-splines basis weighted by their corresponding fitting coefficients are presented in gray lines. 


\section{A.2 COEFFICIENT ESTIMATION}

Once we know how the B-spline basis functions are defined, we need to estimate the coefficients $c_{i}$. To do so, three methods are presented below.

\section{A.2.1 Unweighted least squares fits}

In this simplest approach, we consider that the optimum values of $c_{i}$ coefficients are the ones that minimize the least square criterion.

$\operatorname{SMSSE}(y \mid \mathbf{c})=\sum_{j=1}^{\text {length }(y)}\left[y_{j}-\sum_{i}^{m-n-1} c_{i} \phi_{i}\left(t_{j}\right)\right]^{2}=(\mathbf{y}-\Phi \mathbf{c})^{\prime}(\mathbf{y}-\boldsymbol{\Phi c})$

To find the values of $\hat{\mathbf{c}}$ that minimize the criterion $\operatorname{SMSSE}(y \mid \mathbf{c})$, the derivative of Equation 83 with respect to $c$ has been taken,

$$
2 \Phi \Phi^{\prime} \hat{\mathbf{c}}-2 \Phi^{\prime} \mathbf{y}=0 \rightarrow \hat{\mathbf{c}}=\left(\Phi^{\prime} \Phi\right)^{-1} \Phi^{\prime} \mathbf{y}
$$

and consequently the vector of coefficients $\hat{\mathbf{c}}$ is

$$
\hat{\mathbf{c}}=\left(\Phi^{\prime} \Phi\right)^{-1} \Phi^{\prime} \mathbf{y}
$$

Finally, if we know the coefficients $\hat{\mathbf{c}}$, and the B-spline basis functions $\Phi$, we can easily obtain the vector of fitted values

$$
\mathbf{x}=\Phi \hat{\mathbf{c}}=\Phi\left(\Phi^{\prime} \Phi\right)^{-1} \Phi^{\prime} \mathbf{y}
$$

\section{A.2.2 Weighted least squares fits}

The least squares approximation presented above assumes that the residuals $\epsilon_{j}=s_{j}-y_{j}$ obtained for each point of the curve are i.i.d. with mean zero and constant variance $\sigma^{2}$. To avoid this assumption, the weighted least squares fits was purposed allowing for unequal weighting of squares and products of residuals.

$$
\operatorname{SMSSE}(\mathbf{y} \mid \mathbf{c})=(\mathbf{y}-\boldsymbol{\Phi c})^{\prime} \mathbf{W}(\mathbf{y}-\boldsymbol{\Phi} \mathbf{c})
$$

where $\mathbf{W}$ is a symmetric positive definite matrix. If the variance-covariance matrix $\Sigma_{e}^{-1}$ for the residuals $\epsilon_{j}$ is known, then $\mathbf{W}=\epsilon_{\mathbf{j}}$. 

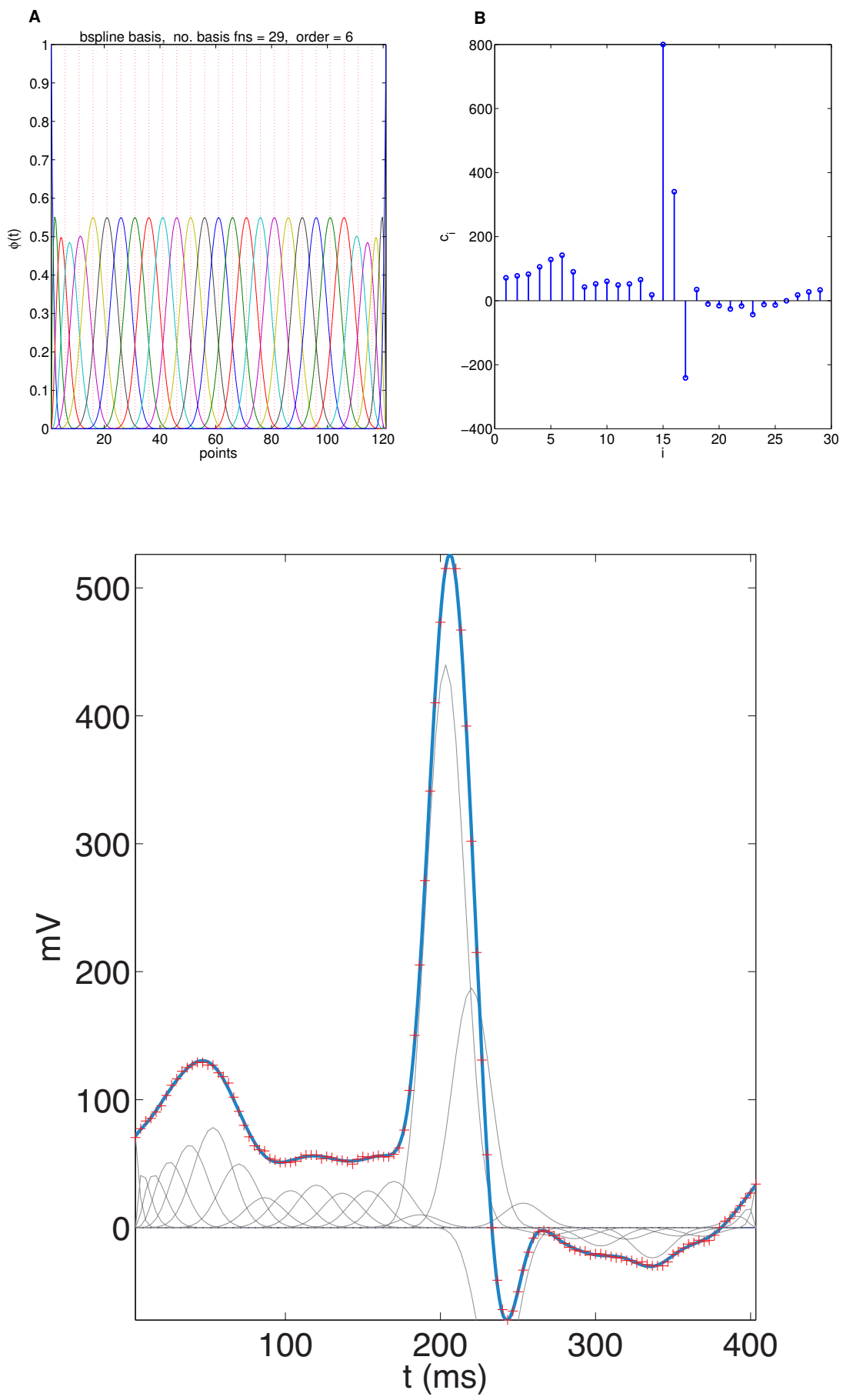

Figure 68: B-spline fitting of a real ECG signal extracted from MIT-BIH public database [MM01, $\mathrm{MSM}^{+}$82]. In A, the B-spline basis functions of order 6 computed using 25 uniformly spaced knots are presented. In $\mathbf{B}$, the values of each of the $\hat{c_{i}}$ coefficients used to fit the ECG signal are presented. Finally in $\mathbf{C}$, the fitting function obtained is presented in blue line. The original ECG signal samples used for the fitting are presented in red crosses, and the B-splines basis weighted by their corresponding fitting coefficients are presented in gray lines. 
Following the same reasoning as above we can obtain the estimate $\hat{\mathbf{c}}$ of the coefficient vector $\mathbf{c}$ for the weighted least squares fits

$$
\hat{\mathbf{c}}=\left(\boldsymbol{\Phi}^{\prime} \mathbf{W} \boldsymbol{\Phi}\right)^{-1} \boldsymbol{\Phi}^{\prime} \mathbf{W} \mathbf{y}
$$

\section{A.2.3 Weighted least squares fits with a roughness penalty}

When fitting the signal it is necessary to take into account the bias between the original signal and the fitting function obtained and also the variance of the fitting function obtained. A completely unbiased estimate of the function value probably will be over-fitted, including high variance, manifested in the rapid local variation of the curve.

In this sense, the quality of estimate is defined by the Mean Squared Error (MSE)

$$
\mathrm{MSE}=\mathrm{Bias}^{2}+\text { Sampling variance }
$$

In order to avoid the over-fitting of the signal and maximize the MSE, a roughness penalty has been introduced. Roughness penalty methods are based on optimizing a fitting criterion that defines the smoothness degree desired for the fitting function.

\section{Quantifying roughness}

The notion of curvature of a function can be used to quantify the roughness of a function. The curvature of a function $x(t)$ in a point $t$ is usually characterized by the square of the second derivative $\left(D^{2} \chi(t)\right)^{2}$ at $t$. Consequently, the measure of a function's roughness can be defined by the integrated squared second derivative

$$
\operatorname{PEN}_{2}(x)=\int\left(D^{2} x(t)\right)^{2} d t
$$

The penalized sum of squared errors fitting criterion

Taking into account the definition of MSE in Equation 89 and the definition of roughness presented in Equation 90, we can modify the last squares fitting criterion so as to allow the roughness penalty to influence in the estimation of the fitting function. That is, the estimate of the function that fits our data is the one that minimize the penalized sum of squared errors $\left(\mathrm{PENSSE}_{2}\right)$ defined by,

$$
\operatorname{PENSSE}_{\lambda}(\mathbf{y} \mid \mathbf{c})=(\mathbf{y}-\mathbf{\Phi c})^{\prime} \mathbf{W}(\mathbf{y}-\mathbf{\Phi c})+\lambda \times \operatorname{PEN}_{2}(x)
$$


The parameter $\lambda$ (smoothing parameter) establish the amount of smoothness of the fitting curve. $\lambda$ defines the compromise between the bias and sampling variance of the fitting function obtained.

\section{Computing splines coefficients}

The roughness penalty $\mathrm{PEN}_{2}(x)$ (see Equation 90 ) can be expressed in matrix terms as follows.

$$
\begin{array}{r}
\operatorname{PEN}_{2}(x)=\int\left[D^{2} \chi(t)\right]^{2} d t \\
=\int\left[D^{2} \mathbf{c}^{\prime} \boldsymbol{\phi}(\mathbf{t})\right]^{2} d t \\
=\int \mathbf{c}^{\prime} D^{2} \boldsymbol{\phi}(\mathbf{t}) D^{2} \boldsymbol{\phi}(\mathbf{t})^{\prime} \mathbf{c d t} \\
=\mathbf{c}^{\prime}\left[\int \mathrm{D}^{2} \boldsymbol{\phi}(\mathbf{t}) \mathrm{D}^{2} \boldsymbol{\phi}(\mathbf{t})^{\prime} \mathrm{ds}\right] \mathbf{c} \\
=\mathbf{c}^{\prime} \mathbf{R} \mathbf{c}
\end{array}
$$

where

$$
R=\int D^{2} \boldsymbol{\phi}(\mathbf{t}) D^{2} \boldsymbol{\phi}(\mathbf{t})^{\prime} d t
$$

Including the expression obtained for $\operatorname{PEN}_{2}(x)$ in 91 we obtain

$$
\operatorname{PENSSE}_{\lambda}(\mathbf{y} \mid \mathbf{c})=(\mathbf{y}-\boldsymbol{\Phi c})^{\prime} \mathbf{W}(\mathbf{y}-\boldsymbol{\Phi c})+\lambda \mathbf{c}^{\prime} \mathbf{R} \mathbf{c}
$$

Minimizing $\operatorname{PENSSE}_{2}(\mathbf{y} \mid \mathbf{c})$ by derivating with respect to parameter vector $\mathbf{c}$, we obtain

$$
2 \Phi^{\prime} \mathbf{W y}+\Phi^{\prime} \mathbf{W} \Phi \mathbf{c}+\lambda \mathbf{R c}=0
$$

from which we obtain the expression for the estimated coefficient vector

$$
\hat{\mathbf{c}}=\left(\boldsymbol{\Phi}^{\prime} \mathbf{W} \boldsymbol{\Phi}+\lambda \mathbf{R}\right)^{-1} \Phi^{\prime} \mathbf{W} \mathbf{y}
$$

In Figure 69, an example of the influence of the $\lambda$ smoothing parameter in the computed fitting function can bee seen. In this example, gaussian noise was added to the original ECG signal samples presented in Figures 67 and 68 for illustration purposes. 

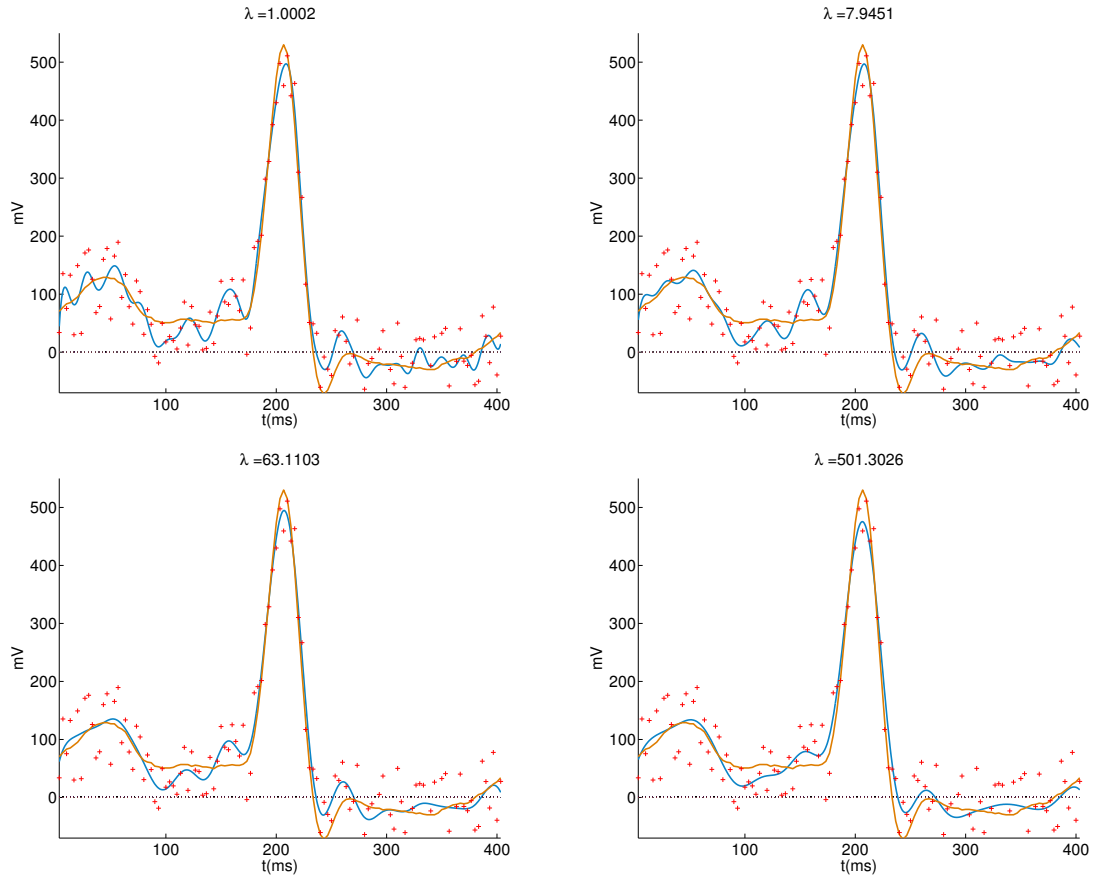

Figure 69: Influence of the $\lambda$ smoothing parameter in the computed fitting function. The original ECG signal samples (with gaussian noise added for illustration purposes) used for estimating the fitting are presented in red crosses. The fitting function obtained is presented in blue line, and the original ECG signal sample (without addition of noise) is presented in yellow. 

Figure 1

Figure 2

Figure 3

Figure 4

Figure 5

Figure 6

Figure 7

Figure 8

Figure 9

Figure 10

Figure 11

Figure 12

Figure 13

Figure 14

Figure 15

Figure 16

Figure 17

Figure 18

Figure 19

Figure 20

Figure 21

Figure 22

Figure 23

Figure 24
Summary of the thesis. 11

Difference of potential energy between levels. 15

RF exitation. 17

The Free Induction Decay (FID). 19

Spin-echo sequence. $\quad 20$

Basic sequence for MRI acquisition. 21

Slice selection. 21

Frequency and phase encoding. $\quad 23$

Example of a K-space and its associated image. $\quad 25$

PRESS sequence diagram. 27

Example of a brain MRS exam. 28

Example of a brain MRSI exam. 28

Metabolites in MRS. $\quad 30$

Phases of the cardiac action potential. 32

Electrical conduction system of the heart. 33

Electrode placements for the limb and augmented limb leads. $\quad 33$

Electrode placements for precordial leads. $\quad 33$

Schematic representation of an standard ECG form a normal heart. $\quad 35$

Wiggers diagram. 36

Back-to-back emission of a pair of $\gamma$ rays in a PET scanner. $\quad 37$

Types of coincidences in PET. $\quad 38$

Image of object slice and their associated sinogram. $\quad 39$

Image reconstruction in PET by filtered backprojection argorithm. 39

Basic steps to build and evaluate a classification model for biomedical signals. 41 
Figure $25 \quad$ Classification boundaries obtained by different classification algorithms. 45

Figure 26 MRSIlab schema 53

Figure 27 MRSI cillindrical phantom 59

Figure 28 Multi-coil MRSI combination by the algorithm for optimal SNR.

Figure 29 K-space apodization functions and their respective PSF 63

Figure 30 MRSIlab data loading window.

Figure 31

MRSIlab main window 68

Figure 32

CURIAM screenshot 71

Figure 33

CURIAM MRS pre-processing pipeline 72

Figure 34 FID signal, FID unsuppressed water signal, ECC signal,residual-water suppressed signal, and apodized signal. $\quad 73$

Figure 35 MRS signal after: baseline offset correction, ULN, frequency shift correction, and truncation to the ROI. $\quad 74$

Figure $36 \quad$ Magnitude response plots of the filters designed to remove ECG artifacts. $\quad 78$

Figure 37

Screenshot of Arrhythlab main window $\quad 80$

Figure 38 Example STE spectra from 1.5 and 3T MR scanners of the two tumour types used in this study. $\quad 87$

Figure 39 Spectral patterns of each of the classes (HGM, CG2G) for the $1.5 \mathrm{~T}$ training, $1.5 \mathrm{~T}$ test and $3 \mathrm{~T}$ test data sets. $\quad 90$

Figure 40 Box-and-whisker diagrams for HGM,CG2G, comparing the main brain metabolite ratios obtained for both field strengths. $\quad 91$

Figure 41 Example of real (gray line) and simulated (black line) MRS data. The real MRS spectrum corresponds to a GBM MRS tumour case. The simulated MRS spectrum have obtained with a $\sigma=0.05$ and without baseline and frequency shift artifacts. $\quad 98$

Figure 42 Mean and deviation of the four real spectra classes. 100

Figure 43 Mean and deviation of the five simulated spectra classes. 101 
Figure 44 Autocorrelation contour plot and mean spectra for CG2G vs MET, MEN vs MET and GBM vs MET binary classification problems. 105

Figure 45 Autocorrelation contour plot and mean spectra for CG2G vs MEN, GBM vs CG2G and GBM vs MEN binary classification problems. 106

Figure 46 Example of the B-spline basis used for GBM vs MET classification problem considered. 109

Figure $47 \quad$ Original mean spectra for the two classes (dotted lines) and their reconstruction using RSFDA (solid lines) for the first three binary classification problems considered: CG2G vs MET, MEN vs MET and GBM vs MET 110

Figure $48 \quad$ Original mean spectra for the two classes (dotted lines) and their reconstruction using RSFDA (solid lines) for the last three binary classification problems considered:CG2G vs MEN, GBM vs $\mathrm{CG} 2 \mathrm{G}$ and GBM vs MEN. 111

Figure 49 Accuracy (ACC) and number of variables obtained in the classification problems using RSFDA, FC, and PCA feature extraction algorithms, with different datasets with different baseline distortion levels. $\quad 115$

Figure 50 Accuracy (ACC) and number of variables obtained in the classification problems using RSFDA, FC, and PCA feature extraction algorithms, with different datasets with different noise levels. 116

Figure 51 Accuracy (ACC) and number of variables obtained in the classification problems using RSFDA, FC, and PCA feature extraction algorithms, with different datasets with different frequency shifts. 117

Figure 52 Input variables for the ANN based sorting method in a PET scanner consisting in 3 axial rings of 32 modules each . 125

Figure 53 Schema of the simulated scanner used in this work (left side) the and phantoms used to evaluate the studied models (right side). 125

Figure 54 Schematic representation of the sorting method based on ANN. 127

Figure 55 Schematic representation of the sorting method based on a fixed time window. 127 
Figure $56 \quad$ RaF vs E performance of the Conventional Sorting method (blue) for different window widths and the ANN sorting method (red) for various thresholds on the Point-like, Line-like, Mouse-like and Rat-like phantoms. 131

Figure $57 \quad$ Reconstructed axial and coronal images for $1 \mathrm{mCi}$ cylindrical phantom using an ideal sorter containing all true coincidences (top), the conventional sorter (middle), and the ANN sorter (bottom). 132

Figure 58 Relative improvement of RSD, Contrast and CNR with the ANN-based method for each number of iterations in four different ROIs 133

Figure 59 ECG patterns of Normal/Supraventricular beats (blue) and Ventricular beats (red), for the different leads used for classification 138

Figure 60

Workflow diagram 139

Figure $61 \quad$ Scatterplot for quality control of ECG data. 140

Figure 62 Scatter plot of the two first principal component coefficients of every beat contained in the SEE dataset. 143

Figure 63 Performance vs. number of variables used by the classifiers. 144

Figure 64 Performances obtained with and without alignment. 145

Figure 65 Mean performances obtained using different FE methods. 146

Figure 66 Mean performances obtained using different classification algorithms. 146

Figure $67 \quad$ Uniform vs. non-uniform B-splines fitting 158

Figure 68 B-splines fitting of a real ECG signal extracted from MIT-BIH public database [MM01, $\mathrm{MSM}^{+}$82] 160

Figure 69 Influence of the $\lambda$ smoothing parameter in the computed fitting function 163 
Table 1

Table 2

Table 3

Table 4

Table 5

Table 6

Table 7

Table 8

Table 9

Table 10

Table 11

Table 12

Table 13

Table 14
Typical metabolite resonances. 31

Combination of electrodes to obtain limb, augmented limb and precordial leads. Note that some leads are known as the same name of the positive electrode that they are obtained from. $\quad 34$

Description of the .SPAR file fields used for MRSI data reading, processing and registration. 55

Description of the .SHF file fields used for MRSI data reading, and processing. $\quad 57$

Position variables obtained from DICOM MRI images and a brief description. $\quad 65$

Beat tags used in Arrhythlab. $\quad 79$

Event tags used in Arrhythlab. $\quad 79$

Number of 1H SV-MRS spectra in each dataset per tumour type. $\quad 86$

Classification results obtained for the three datasets: 1.5T train (CV), 1.5T test, and 3T test. 91

Number of ${ }^{1} \mathrm{H}$ SV-MRS real spectra in each multicenter dataset per tumor type. $\quad 97$

Amplitude values $\bar{a}$ of metabolites and other molecules observed in Short TE for for each simulated classes. 100

The performance results in terms of $\mathrm{G} \pm \sigma$ for each of the six binary classification problems considered based on the multicenter brain tumor MRS datasets. In the first table, the results presented have been obtained training the classification model with INTERPRET dataset and testing them with eTUMOUR dataset. In the second table, the results presented have been obtained training the classification models with eTUMOUR dataset and testing them with INTERPRET dataset. 119

Values of Random Fraction (RaF) for equal Efficiency (E). 130

Distribution of patients and beats per pathology. 
Table $15 \quad$ Performance obtained for each combination of FE and classification algorithm in terms of $\mathrm{G}$ values. The results were obtained using the first 64 features $(n=64)$ extracted by each FE algorithm from the aligned SEE dataset. 147 
[Ass00] N. E. M. Association. Digital imaging and communications in medicine (DICOM) part 5: Data structures and encoding. Technical Report PS 3.3-2009, Rosslyn, Virginia 22209 USA, 2000.

[AVR12] C. Arizmendi, A. Vellido, and E. Romero. Classification of human brain tumours from mrs data using discrete wavelet transform and bayesian neural networks. Expert Syst. Appl., 39(5):5223-5232, April 2012.

[BDC76] R. A. Brooks and G. Di Chiro. Principles of computer assisted tomography (cat) in radiographic and radioisotopic imaging. Physics in Medicine and Biology, 21(5):689-732, 1976.

[BE07] E. S. Berner and (Ed.). Clinical Decision Support Systems: Theory and Practice. Springer New York, 2007.

[BHB01] P. B. Barker, D. O. Hearshen, and M. D. Boska. Singlevoxel proton mrs of the human brain at $1.5 \mathrm{t}$ and $3.0 \mathrm{t}$. Magnetic Resonance in Medicine, 45(5):765-769, 2001.

[Bis95] C. M. Bishop. Neural Networks for Pattern Recognition. Oxford University Press, November 1995.

[Bra02] D. Brand. Electronic decision support for australia's health sector. Technical report, National Electronic Support Taskforce, 2002.

[Bur04] C. J. Burges. Geometric methods for feature extraction and dimensional reduction: A guided tour. Technical report, Microsoft Research, University of Toronto, 2004.

[CBV11] R. Cruz-Barbosa and A. Vellido. Semi-supervised analysis of human brain tumours from partially labeled mrs information, using manifold learning models. Int. J. Neural Syst., 21(1):17-29, 2011.

$\left[\mathrm{CCGLF}^{+}\right.$01] E. Cabanes, S. Confort-Gouny, Y. Le Fur, G. Simond, and P. J. Cozzone. Optimization of residual water signal removal by HLSVD on simulated short echo time proton MR spectra of the human brain. J Magn Reson, 150(2):116125, Jun 2001. 
$\left[\mathrm{CMMB}^{+}\right.$06] B. Celda, D. Monleón, M. C. Martínez-Bisbal, V. Esteve, B. Martínez-Granados, E. Piñero, R. Ferrer, J. Piquer, L. Martí-Bonmatí, and J. Cervera. Mrs as endogenous molecular imaging for brain and prostate tumors: Fp6 project "etumor". Adv Exp Med Biol, 587:285-302, 2006.

[Coh00] A. Cohen. Biomedical Signals: Origin and Dynamic Characteristics; Frequency-Domain Analysis. In J. D. Bronzino, editor, The Biomedical Engineering HandBook. CRC Press LLC, second edition edition, 2000.

[Com79] D. S. P. Committee. Programs for Digital Signal Processing. IEEE Press, New York, 1979.

[CON09] J. C. Crane, M. P. Olson, and S. J. Nelson. An Extensible Open-Source DICOM MR Spectroscopy Software Framework and Application Suite. In Book of Abstracts: International Conference on Magnetic Resonance in Medicine, number 3354, 2009.

[CW79] P. Craven and G. Wahba. Smoothing noisy data with spline functions: Estimating the correct degree of smoothing by the method of generalized cross-validation. Numerische Mathematik, 31(4):377-403, 1979.

[Dau92] I. Daubechies. Ten Lectures on Wavelets (CBMS - NSF Regional Conference Series in Applied Mathematics). Soc for Industrial \& Applied Math, December 1992.

[Deb78] C. Deboor. A Practical Guide to Splines. Springer-Verlag Berlin and Heidelberg GmbH \& Co. K, December 1978.

[dGvDB90] A. A. de Graaf, J. E. van Dijk, and W. M. Bovee. QUALITY: quantification improvement by converting lineshapes to the Lorentzian type. Magn Reson Med, 13(3):343-357, Mar 1990.

[DHS00] R. O. Duda, P. E. Hart, and D. G. Stork. Pattern Classification (2nd Edition). Wiley-Interscience, New York, November 2000.

$\left[\mathrm{DLS}^{+}\right.$04] A. Devos, L. Lukas, J. A. K. Suykens, L. Vanhamme, A. R. Tate, F. A. Howe, C. Majos, A. Moreno-Torres, M. van der Graaf, C. Arús, and S. Van Huffel. Classification of brain tumours using short echo time $1 \mathrm{H}$ MR spectra. Journal of Magnetic Resonance, 170(1):164-175, Sep 2004.

[DMLB06] U. Dydak, D. Meier, R. Lamerichs, and P. Boesiger. Effect of feature extraction for brain tumor classification based on short echo time $1 \mathrm{~h}$ mr spectra. Am J Neuroradiol, 27:14416, 2006. 
[EBW87] R. R. Ernst, G. Bodenhausen, and A. Wokaun. Principles of Nuclear Magnetic Resonance in one and two dimensions. Clerendon Press, Oxford, 1987.

[eTU] eTUMOUR Consortium. eTumour: Web accessible MR Decision support system for brain tumour diagnosis and prognosis, incorporating in vivo and ex vivo genomic and metabolomic data. Web site. FP6-2002LIFESCIHEALTH 503094, VI framework programme, EC, http://www.etumour.net (Accessed: 15th January 2011).

[Fah02] F. Fahey. Data acquisition in pet imaging. J Nucl Med Technol, 30(2):39-49, 2002.

[FGAG $\left.{ }^{+} 11\right]$ E. Fuster-Garcia, D. Arazo, D. Goldwasser, M. Hurtado, J. M. Doña, T. Salvador, J. M. García Gómez, and G. Serra. Identificación de puntos significativos para el estudio de arritmias en electrocardiogramas. In Book of Abstracts Congreso de las Enfermedades Vasculares SEC. Maspalomas Gran Canaria, page 894, 2011.

$\left[\mathrm{FGNV}^{+} 11\right]$ E. Fuster-Garcia, C. Navarro, J. Vicente, S. Tortajada, J. M. García-Gómez, C. Sáez, J. Calvar, J. Griffiths, M. JuliàSapé, F. a. Howe, J. Pujol, A. C. Peet, A. Heerschap, A. Moreno-Torres, M. C. Martínez-Bisbal, B. MartínezGranados, P. Wesseling, W. Semmler, J. Capellades, C. Majós, A. Alberich-Bayarri, A. Capdevila, D. Monleón, L. Martí-Bonmatí, C. Arús, B. Celda, and M. Robles. Compatibility between $3 \mathrm{~T} 1 \mathrm{H}$ SV-MRS data and automatic brain tumour diagnosis support systems based on databases of 1.5T 1H SV-MRS spectra. Magnetic Resonance Materials in Physics, Biology and Medicine, 24(1):35-42, February 2011.

$\left[\mathrm{FGOC}^{+}\right.$10a] E. Fuster-Garcia, J. Oliver, J. Cabello, S. Tortajada, and M. Rafecas. Reduction of random coincidences in small animal pet using artificial neural networks. In Nuclear Science Symposium Conference Record (NSS/MIC), 2010 IEEE, pages 2308 -2313, 30 2010-nov. 62010.

$\left[\mathrm{FGOC}^{+}\right.$10b] E. Fuster-Garcia, J. Oliver, J. Cabello, S. Tortajada, and M. Rafecas. Reduction of random coincidences in small animal pet using artificial neural networks. In IEEE Nuclear Science Symposium Conference Record, pages 2308 - 2313, 2010.

[FGOTR08] E. Fuster-Garcia, J. Oliver, S. Tortajada, and M. Rafecas. Coincidence identification in pet using neural network. In IEEE Nuclear Science Symposium Conference Record, pages 4728-4731, October 2008. 
[Fis36] R. A. Fisher. The Use of Multiple Measurements in Taxonomic Problems. Ann Eugenic, 7:179-188, Sep 1936.

[Fuk90] K. Fukunaga. Introduction to statistical pattern recognition. Academic Press Professional, Inc., San Diego, CA, USA, second edition edition, 1990.

[Gad95] D. Gadian. Nuclear Magnetic Resonance and its Applications to Living Systems. Oxford: Clarendon Press, 1995.

[GC00] C. Giraud-Carrier. A note on the utility of incremental learning. AI Communications, 13:215-223, 2000.

[GGEJS ${ }^{+}$09] J. M. García-Gómez, I. Epifanio, M. Julà-Sapé, D. Monleón, J. Vicente, S. Tortajada, E. Fuster, A. Moreno-Torres, A. Peet, F. Howe, B. Celda, C. Arús, and M. Robles. Possibilistic classification of brain tumors by mrs based on functional data analysis and subpattern discovery. In Proceedings 17th Scientific Meeting, International Society for Magnetic Resonance in Medicine, Honolulu, page 146, 2009.

[GGL $\left.{ }^{+} 01\right]$ O. Gonen, S. Gruber, B. Li, V. Mlynárik, and E. Moser. Multivoxel 3d proton spectroscopy in the brain at 1.5 versus 3.0 t: signal-to-noise ratio and resolution comparison. Am J Neuroradiol., 22:1727-31, Oct 2001.

[GGLJS ${ }^{+}$08] J. M. García-Gómez, J. Luts, M. Julià-Sapé, P. Krooshof, S. Tortajada, J. V. Robledo, W. Melssen, E. Fuster-Garcia, I. Olier, G. Postma, D. Monleon, A. Moreno-Torres, J. Pujol, A.-P. Candiota, M. C. Martinez-Bisbal, J. Suykens, L. Buydens, B. Celda, S. Van Huffel, C. Arus, and M. Robles. Multiproject-multicenter evaluation of automatic brain tumor classification by magnetic resonance spectroscopy. In S. B. Heildelberg, editor, Book of Abstracts: 25th Annual Scientific Meeting of the European Society of Magnetic Resonance in Medicine and Biology, 2008.

[GGLJS ${ }^{+}$09] J. M. García-Gómez, J. Luts, M. Julià-Sapé, P. Krooshof, S. Tortajada, J. Vicente, W. Melssen, E. Fuster-Garcia, I. Olier, G. Postma, D. Monleón, A. Moreno-Torres, J. Pujol, A.-P. Candiota, M. C. Martínez-Bisbal, J. Suykens, L. Buydens, B. Celda, S. Van Huffel, C. Arús, and M. Robles. Multiproject multicenter evaluation of automatic brain tumor classification by magnetic resonance spectroscopy. Magnetic Resonance Materials in Physics, Biology and Medicine, 22(1):5-18, 2009.

$\left[\mathrm{GGTV}^{+}\right.$08] J. M. García-Gómez, S. Tortajada, C. Vidal, M. Julia-Sape, J. Luts, S. Van Huffel, C. Arús, and M. Robles. The influence of combining two echo times in automatic brain 
tumor classification by magnetic resonance spectroscopy. NMR in Biomedicine, 21(10):1112-1125, 2008.

[GGVFRV05] J. M. García-Gómez, C. Vidal-Fernández, and M. RoblesViejo. Preliminary choice of pattern recognition techniques and methodology, discussion on implementation details. Technical report, BET-IM, Universidad Politécnica de Valencia, 2005.

[GGVRRV05] J. M. García-Gómez, J. Vicente-Robledo, and M. RoblesViejo. Implementation of pattern recognition methods for clinical, histological, metabolic and genetic data vs. $\mathrm{mri} / \mathrm{mrs}$ information. Technical report, BET-IM, Universidad Politécnica de Valencia, 2005.

$\left[\mathrm{GNBMnR}^{+}\right.$10] F. F. González-Navarro, L. A. Belanche-Muñoz, E. Romero, A. Vellido, M. Julií-Sapé, and C. Arús. Feature and model selection with discriminatory visualization for diagnostic classification of brain tumors. Neurocomput., 73(4-6):622632, January 2010.

$\left[\mathrm{GPF}^{+}\right.$04] Y. Goletsis, C. Papaloukas, D. I. Fotiadis, A. Likas, and L. K. Michalis. Automated ischemic beat classification using genetic algorithms and multicriteria decision analysis. IEEE transactions on bio-medical engineering, 51(10):1717-25, October 2004.

[Gri11] S. L. Grime. Using 80001 to manage medical devices on the it network. IT Horizons, 6:23-26, 2011.

[GVMJS ${ }^{+}$07] H. González-Vélez, M. Mier, M. Julià-Sapé, T. N. Arvanitis, J. M. García-Gómez, M. Robles, P. H. Lewis, S. Dasmahapatra, D. Dupplaw, A. Peet, C. Arús, B. Celda, S. V. Huffel, and M. Lluch i Ariet. HealthAgents: Distributed multi-agent brain tumour diagnosis and prognosis. Journal of Applied Intelligence, 30(3):191-202, 2007.

[HKO01] A. Hyvärinen, J. Karhunen, and E. Oja. Independent Component Analysis. Wiley-Interscience, 1 edition, May 2001.

[HPR04] N. P. Hughes, N. Peter, and S. J. Roberts. Markov models for automated ECG interval analysis. In In Proceedings of the Advances in Neural Information Processing Systems 16, pages 611-618. MIT Press, 2004.

$\left[\mathrm{HRF}^{+}\right.$08] E. Hattingen, P. Raab, K. Franz, H. Lanfermann, M. Setzer, R. Gerlach, F. Zanella, and U. Pilatus. Prognostic value of choline and creatine in who grade ii gliomas. Neuroradiology, 50:759-67, Sep 2008. 
[HRM04] G. M. Hackbarth, R. Reischauer, and M. E. Miller. Report to the congress: New approaches in medicare. Technical report, Medicare Payment Advisory Commission, Washington, EEUU, 2004.

[HT86] P. S. Hamilton and W. J. Tompkins. Quantitative investigation of QRS detection rules using the MIT/BIH arrhythmia database. IEEE Trans Biomed Eng, 33(12):1157-1165, December 1986.

[INT08] INTERPRET Consortium. Interpret web site, Accessed: 28 April 2008. http://azizu.uab.es/INTERPRET.

[JDC87] A. K. Jain, R. C. Dubes, and C. Chen. Bootstrap techniques for error estimation. IEEE Transactions on Pattern Analysis and Machine Intelligence, 9(5):628-633, 1987.

[Jol02] I. T. Jolliffe. Principal Component Analysis. Springer, second edition, October 2002.

[JSAM ${ }^{+}$06] M. Julià-Sapé, D. Acosta, M. Mier, C. Arús, and D. Watson. A multi-centre, web-accessible and quality control-checked database of in vivo $\mathrm{mr}$ spectra of brain tumour patients. Magnetic Resonance Materials in Physics, Biology and Medicine, 19(1):22-33, 2006. 10.1007/s10334-005-0023$\mathrm{x}$.

$\left[\mathrm{KCN}^{+}\right.$06] J. Kim, K. Chang, D. G. Na, I. C. Song, S. J. Kim, B. J. Kwon, and M. H. Han. Comparison of $1.5 \mathrm{~T}$ and $3 \mathrm{~T} 1 \mathrm{H}-$ MR spectroscopy for human brain tumors. Korean J Radiol, 7(3):156-161, 2006.

[Klo90] U. Klose. In vivo proton spectroscopy in presence of eddy currents. Magn Reson Med, 14(1):26-30, April 1990.

$\left[\mathrm{KMN}^{+} 06\right]$ B. M. Kelm, B. H. Menze, T. Neff, C. M. Zechmann, and F. A. Hamprecht. CLARET: a tool for fully automated evaluation of MRSI with pattern recognition methods. In H. Handels, J. Ehrhardt, A. Horsch, H.-P. Meinzer, and T. Tolxdorff, editors, Bildverarbeitung für die Medizin, Informatik Aktuell, pages 51-55. Springer, 2006.

[Koh95] R. Kohavi. A study of cross-validation and bootstrap for accuracy estimation and model selection. In IJCAI, pages 1137-1145, 1995.

[KSS97] I. Kononenko, E. Simec, and M. R. Sikonja. Overcoming the Myopia of Inductive Learning Algorithms with RELIEFF. Applied Intelligence, 7(1):39-55, 1997. 
[KWS ${ }^{+}$09] T. Küstner, J. Weidendorfer, J. Schirmer, T. Klug, C. Trinitis, and S. Ziegler. Parallel mlem on multicore architectures. In Proceedings of 9th International Conference on Computational Science, pages 491-500. Springer, 2009.

[LC89] K. P. Lin and W. H. Chang. QRS feature extraction using linear prediction. IEEE transactions on bio-medical engineering, 36(10):1050-5, October 1989.

$\left[\operatorname{LDS}^{+} 04\right]$ L. Lukas, A. Devos, J. A. K. Suykens, L. Vanhamme, F. A. Howe, C. Majós, A. Moreno-Torres, M. Van der Graaf, A. R. Tate, C. Arús, and S. Van Huffel. Brain tumor classification based on long echo proton MRS signals. Artificial intelligence in medicine, 31(1):73-89, May 2004.

[LMBCV08] T. Laudadio, M. C. Martínez-Bisbal, B. Celda, and S. Van Huffel. Fast nosological imaging using canonical correlation analysis of brain data obtained by two-dimensional turbo spectroscopic imaging. NMR Biomed., 21:311-321, May 2008.

$\left[\mathrm{LPB}^{+} 00\right]$ M. Lagerholm, C. Peterson, G. Braccini, L. Edenbrandt, and L. Sörnmo. Clustering ECG complexes using hermite functions and self-organizing maps. IEEE transactions on bio-medical engineering, 47(7):838-48, July 2000.

[LPGG ${ }^{+}$08] J. Luts, J. B. Poullet, J. M. Garcia-Gomez, A. Heerschap, M. Robles, J. A. K. Suykens, and S. Van Huffel. Effect of feature extraction for brain tumor classification based on short echo time 1H MR spectra. Magnetic Resonance in Medicine, 60(2):288-98, August 2008.

$\left[\mathrm{LPN}^{+} 10\right]$ E. W. Lau, R. K. Pathamanathan, G. A. Ng, J. Cooper, J. D. Skehan, and M. J. Griffith. The Bayesian approach improves the electrocardiographic diagnosis of broad complex tachycardia. Pacing and Clinical Electrophysiology: PACE, 23(10 Pt 1):1519-1526, 2010.

[LPS05] L. Landini, V. Positano, and M. Santarelli. Advanced image processing in magnetic resonance imaging. Signal processing and communications. CRC/Taylor \& Francis, 2005.

$\left[\mathrm{MAA}^{+}\right.$05] S. Mahmoodabadi, A. Ahmadian, M. Abolhasani, M. Eslami, and J. Bidgoli. ECG Feature Extraction Based on Multiresolution Wavelet Transform. Conference proceedings : Annual International Conference of the IEEE Engineering in Medicine and Biology Society. IEEE Engineering in Medicine and Biology Society. Conference, 4:3902-5, January 2005. 
[Mac92] D. J. C. Mackay. A practical Bayesian framework for backpropagation networks. Neural Computation, 4:448$472,1992$.

$\left[\mathrm{MBCMB}^{+}\right.$02] M. Martínez-Bisbal, B. Celda, L. Martí-Bonmatí, P. Ferrer, A. Revert, J. Piquer, E. Mollá, E. Arana, and R. Dosdá. Contribution of magnetic resonance spectroscopy to the classification of hogh glial tumours. predictive value of macromolecules. Rev Neurol., 34:309-313, Feb 2002.

[MdS10] P. S. e. I. Ministerio de Sanidad. Plan de calidad para el sistema nacional de salud. Technical report, Madrid, Spain, 2010.

$\left[\mathrm{MHP}^{+}\right.$05a] D. McElroy, M. Hoose, W. Pimpl, V. C. Spanoudaki, T. Schüler, and S. I. Ziegler. A true singles list-mode data acquisition system for a small animal pet scanner with independent crystal readout. Physics in Medicine and Biology, 50(14):3323-3335, 2005.

[MHP ${ }^{+}$05b] D. P. McElroy, M. Hoose, W. Pimpl, V. Spanoudaki, T. Schüler, and S. I. Ziegler. A true singles list-mode data acquisition system for a small animal pet scanner with independent crystal readout. Physics in Medicine and Biology, 50(14):3323, 2005.

$\left[\mathrm{MLB}^{+} 06\right]$ B. H. Menze, M. P. Lichy, P. Bachert, B. M. Kelm, H.-P. Schlemmer, and F. A. Hamprecht. Optimal classification of long echo time in vivo magnetic resonance spectra in the detection of recurrent brain tumors. NMR in biomedicine, 19(5):599-609, August 2006.

[MM01] G. B. Moody and R. G. Mark. The impact of the MIT$\mathrm{BIH}$ arrhythmia database. IEEE engineering in medicine and biology magazine : the quarterly magazine of the Engineering in Medicine \& Biology Society, 20(3):45-50, 2001.

[Mon08] M. Montelius. Matlab tool for segmentation and re-creation of ${ }^{1} \mathrm{H}$-MRS volumes of interest in MRI image stacks. Master's thesis, Göteborg University, Sweden, 2008.

$\left[\mathrm{MSM}^{+}\right.$82] R. G. Mark, P. S. Schluter, G. B. Moody, P. H. Devlin, and D. Chernoff. An annotated ecg database for evaluating arrhythmia detectors. IEEE Transactions on Biomedical Engineering, 29(8):600, 1982.

[OHM04] S. Osowski, L. T. Hoai, and T. Markiewicz. Support vector machine-based expert system for reliable heartbeat recognition. IEEE transactions on bio-medical engineering, 51(4):582-9, April 2004. 
$\left[\mathrm{OLB}^{+}\right.$07] K. S. Opstad, C. Ladroue, B. A. Bell, J. R. Griffiths, and F. A. Howe. Linear discriminant analysis of brain tumour 1H MR spectra: a comparison of classification using whole spectra versus metabolite quantification. NMR in Biomedicine, 20(8):763-770, 2007.

$\left[\mathrm{OmOV}^{+} 10\right]$ S. Ortega-martorell, I. Olier, A. Vellido, M. Julià-sapé, and C. Arús. Spectral prototype extraction for dimensionality reduction in brain tumour diagnosis. In ESANN 2010 proceedings, European Symposium on Artificial Neural Networks Computational Intelligence and Machine Learning., 2010.

$\left[\mathrm{OPB}^{+} 09\right]$ K. Opstad, S. Provencher, B. Bell, J. Griffiths, and F. Howe. Detection of elevated glutathione in meningiomas by quantitative in vivo $1 \mathrm{~h}$ mrs. Magn Reson Med., 49:632-7, Apr 2009.

[PCB82] R. Prineas, R. Crow, and H. Blackburn. The Minnesota code manual of electrocardiographic findings: standards and procedures for measurement and classification. J. Wright, 1982.

[Pea01] K. Pearson. On lines and planes of closest fit to systems of points in space. Philosophical Magazine, 2(6):559-572, 1901.

[Ped09] P. Pedron. Implementation of an analytical 3d model for generating the system matrix of a pet scanner. Fakultät Für Informatik, Technische Universität München, 2009.

[PFLM02] C. Papaloukas, D. I. Fotiadis, A. Likas, and L. K. Michalis. An ischemia detection method based on artificial neural networks. Artificial intelligence in medicine, 24(2):167-78, 2002.

[PM10] E. Pasolli and F. Melgani. Active learning methods for electrocardiographic signal classification. IEEE Trans Inf Technol Biomed., 14(6):1405-1416, November 2010.

[Pra06] P. Prasad. Magnetic Resonance Imaging: Methods And Biologic Applications. Methods in Molecular Medicine. Humana Press, 2006.

[PRJSM $^{+}$10] A. Perez-Ruiz, M. Julia-Sape, G. Mercadal, I. Olier, C. Majos, and C. Arus. The interpret decision-support system version 3.0 for evaluation of magnetic resonance spectroscopy data from human brain tumours and other abnormal brain masses. BMC Bioinformatics, 11(1):581, 2010. 
[Pro01] S. W. Provencher. Automatic quantitation of localized in vivo $1 \mathrm{H}$ spectra with LCModel. NMR in Biomedicine, 14:260-264, 2001.

[PROCJS ${ }^{+}$08] A. Pérez-Ruiz, I. A. Olier-Caparroso, M. Julià-Sapé, A. P. Candiota, and C. Arús. Brain Tumor Diagnosis with MRS: The Single Voxel INTERPRET Decision-Support System version 2.0. In Book of abstracts ESMRMB 2008: 25th Annual Scientific Meeting of the European Society for Magnetic Resonance in Medicine and Biology. SpringerVerlag, Oct 2008.

[PSS ${ }^{+}$07] J.-B. Poullet, D. M. Sima, A. W. Simonetti, B. D. Neuter, and L. Vanhamme. An automated quantitation of short echo time MRS spectra in an open source software environment : AQSES. NMR in Biomedicine, 20(5):493-504, 2007.

$\left[\mathrm{REH}^{+}\right.$90] P. B. Roemer, W. A. Edelstein, C. E. Hayes, S. P. Souza, and O. M. Mueller. The NMR phased array. Magnetic Resonance in Medicine, 16(2):192-225, 1990.

[RFGOH12] F. Raschke, E. Fuster-Garcia, K. S. Opstad, and F. a. Howe. Classification of single-voxel (1) $\mathrm{H}$ spectra of brain tumours using LCModel. NMR in biomedicine, 25(2):322-331, 2012.

[RHM ${ }^{+}$97] W. Roser, G. Hagberg, I. Mader, S. Dellas, J. Seelig, E. Radue, and W. Steinbrich. Assignment of glial brain tumors in humans by in vivo 1h-magnetic resonance spectroscopy and multidimensional metabolic classification. Magn Reson Mater Phy, 5:179-183, 1997.

[RN95] S. J. Russell and P. Norvig. Artificial intelligence: a modern approach. Prentice-Hall, Inc., Upper Saddle River, NJ, USA, 1995.

$\left[\mathrm{RRL}^{+}\right.$06] C. Roever, N. Raabe, K. Luebke, U. Ligges, G. Szepannek, and M. Zentgraf. The klaR Package. Deparment of Statistics, University of Dortmund, January 2006.

[RS05] J. O. Ramsay and B. W. Silverman. Functional Data Analysis. Springer Series in Statistics. Springer, 2nd edition, June 2005.

[RSC ${ }^{+}$05] H. Ratiney, M. Sdika, Y. Coenradie, S. Cavassila, D. van Ormondt, and D. Graveron-Demilly. Time-domain semiparametric estimation based on a metabolite basis set. NMR in biomedicine, 18(1):1-13, February 2005.

$\left[\mathrm{SBL}^{+} 05\right]$ M. Streun, G. Brandenburg, H. Larue, C. Parl, and K. Ziemons. The data acquisition system of clearpet/spl 
trade/ neuro - a small animal pet scanner. Book of abstracts: IEEE-NPSS Real Time Conference, 0:21, 2005.

[SGG ${ }^{+}$01] I. Sim, P. Gorman, R. a. Greenes, R. B. Haynes, B. Kaplan, H. Lehmann, and P. C. Tang. Clinical decision support systems for the practice of evidence-based medicine. Journal of the American Medical Informatics Association, 8(6):52734, 2001.

[SGGAB $\left.{ }^{+} 12\right]$ C. Sáez, J. M. García-Gómez, Á. Alberich-Bayarri, M. Á. Edo, M. Vañó, A. Catalá-Gregori, C. Barber, C. Poyatos, E. Mollá, L. Martí-Bonmatí, and M. Robles. Clinical validation of the added value of a clinical decision support system for brain tumour diagnosis based on sv $1 \mathrm{~h}$ mrs: randomized controlled trial of effectiveness and qualitative evaluation. In 24th European Medical Informatics Conference MIE2012, Pisa, Italy, 2012.

[SGGV ${ }^{+}$08] C. Sáez, J. García-Gómez, J. Vicente, S. Tortajada, M. Esparza, A. Navarro, E. Fuster-Garcia, M. Robles, L. MartíBonmatí, and C. Arús. A generic decision support system featuring an assembled view of predictive models for magnetic resonance and clinical data. In Book of abstracts ESMRMB 2008: 25th Annual Scientific Meeting of the European Society for Magnetic Resonance in Medicine and Biology, page 483. Springer-Verlag, 2008.

$\left[\mathrm{SGGV}^{+}\right.$09] C. Sáez, J. García-Gómez, J. Vicente, S. Tortajada, E. Fuster, M. Esparza, A. Navarro, and M. Robles. Curiam BT 1.0, Decision Support System for Brain Tumour Diagnosis. In Book of Abstracts: 26th Annual Scientific Meeting of the European Society for Magnetic Resonance in Medicine and Biology (ESMRMB), page 538, Antalya, Turkey, may 2009. Springer Berlin / Heidelberg.

[SIL01] B. Sierra, I. Inza, and P. Larrañaga. On applying supervised classification techniques in medicine. In J. Crespo, V. Maojo, and F. Martin, editors, Book of Abstracts: Second International Symposium on Medical Data Analysis, volume 2199 of Lecture Notes in Computer Science, pages 14-19. Springer Berlin / Heidelberg, 2001.

[SJB08] A. Skoch, F. Jiru, and J. Bunke. Spectroscopic imaging: basic principles. European Journal of Radiology, 67(2):230 239, 2008.

$\left[\mathrm{SJK}^{+} 10\right]$ A. Server, R. Josefsen, B. Kulle, J. Maehlen, T. Schellhorn, Ø. Gadmar, T. Kumar, M. Haakonsen, C. Langberg, and P. Nakstad. Proton magnetic resonance spectroscopy in 
the distinction of high-grade cerebral gliomas from single metastatic brain tumors. Acta Radiol., 51:316-25, Apr 2010 .

[SM98] R. Silipo and C. Marchesi. Artificial neural networks for automatic ECG analysis. IEEE Transactions on Signal Processing, 46(5):1417-1425, May 1998.

$\left[\mathrm{SMdE}^{+}\right.$05] A. W. Simonetti, W. J. Melssen, F. S. de Edelenyi, J. J. A. van Asten, A. Heerschap, and L. M. C. Buydens. Combination of feature-reduced MR spectroscopic and MR imaging data for improved brain tumor classification. NMR in Biomedicine, 18(1):34-43, 2005.

[SNE ${ }^{+}$03] P. Sundgren, V. Nagesh, A. Elias, C. Tsien, L. Junck, D. Gomez Hassan, T. Lawrence, T. Chenevert, L. Rogers, P. McKeever, and Y. Cao. Metabolic alterations: a biomarker for radiation-induced normal brain injury-an $\mathrm{mr}$ spectroscopy study. J Magn Reson Imaging, 29:291-7, Feb 2003.

$\left[\mathrm{SSD}^{+}\right.$03] D. Strul, R. B. Slates, M. Dahlbom, S. R. Cherry, and P. K. Marsden. An improved analytical detector response function model for multilayer small-diameter pet scanners. Physics in Medicine and Biology, 48(8):979, 2003.

[TERS ${ }^{+}$08] I. Torres-Espallardo, M. Rafecas, V. C. Spanoudaki, D. McElroy, and S. I. Ziegler. Effect of inter-crystal scatter on estimation methods for random coincidences and subsequent correction. Physics in Medicine and Biology, 53:2391-2411, 2008.

$\left[\mathrm{TFGV}^{+}\right.$11] S. Tortajada, E. Fuster-Garcia, J. Vicente, P. Wesseling, F. a. Howe, M. Julià-Sapé, A.-P. Candiota, D. Monleón, A. Moreno-Torres, J. Pujol, J. R. Griffiths, A. Wright, A. C. Peet, M. C. Martínez-Bisbal, B. Celda, C. Arús, M. Robles, and J. M. García-Gómez. Incremental Gaussian Discriminant Analysis based on Graybill and Deal weighted combination of estimators for brain tumour diagnosis. Journal of biomedical informatics, 44(4):677-87, August 2011.

[The07] The eTUMOUR Consortium. eTUMOUR: Web accessible MR decision support system for brain tumour diagnosis and prognosis, incorporating in vivo and ex vivo genomic and metabolomic data, vi framework programme, ec, http://www.etumour.net. Technical Report FP6-2002LIFESCIHEALTH 503094, February 2007. 
[TK06] S. Theodoridis and K. Koutroumbas. Pattern Recognition, Third Edition. Academic Press, San Diego, February 2006.

[TKST08] E. M. Tamil, N. H. Kamarudin, R. Salleh, and A. M. Tamil. A Review on Feature Extraction \& Classification Techniques for Biosignal Processing (Part I: Electrocardiogram). pages 107-112, 2008.

$\left[\mathrm{TLV}^{+} 05\right]$ M. A. Tetrault, M. D. Lepage, N. Viscogliosi, F. Belanger, J. Cadorette, C. M. Pepin, R. Fontaine, and R. Lecomte. Real time coincidence detection system for digital high resolution apd-based animal pet scanner. Book of abstracts: IEEE-NPSS Real Time Conference, 5:2849-2853, 2005.

$\left[\mathrm{TMM}^{+}\right.$03] A. R. Tate, C. Majos, A. Moreno, F. A. Howe, J. R. Griffiths, and C. Arús. Automated classification of short echo time in in vivo $1 \mathrm{H}$ brain tumor spectra: a multicenter study. Magnetic Resonance in Medicine, 49(1):29-36, Jan 2003.

$\left[\mathrm{TUA}^{+}\right.$06] A. R. Tate, J. Underwood, D. M. Acosta, M. Julia-Sape, C. Majos, A. Moreno-Torres, F. A. Howe, M. van der Graaf, V. Lefournier, M. M. Murphy, A. Loosemore, C. Ladroue, P. Wesseling, J. Luc Bosson, M. E. Cabanas, A. W. Simonetti, W. Gajewicz, J. Calvar, A. Capdevila, P. R. Wilkins, B. A. Bell, C. Remy, A. Heerschap, D. Watson, J. R. Griffiths, and C. Arús. Development of a decision support system for diagnosis and grading of brain tumours using in vivo magnetic resonance single voxel spectra. NMR in Biomedicine, 19(4):411-434, Jun 2006.

[TVR ${ }^{+}$08] M.-A. Tetrault, N. Viscogliosi, J. Riendeau, F. Belanger, J.B. Michaud, H. Semmaoui, P. Berard, F. Lemieux, L. Arpin, M. Bergeron, J. Cadorette, C. M. Pepin, G. Robert, M. D. Lepage, R. Lecomte, and R. Fontaine. System Architecture of the LabPET Small Animal PET Scanner. IEEE Transactions on Nuclear Science, 55:2546-2550, 2008.

[VdGJSH ${ }^{+}$08] M. Van der Graaf, M. Juliá-Sapè, F. A. Howe, A. Ziegler, C. Majós, A. Moreno-Torres, M. Rijpkema, D. Acosta, K. S. Opstad, Y. Van der Meulen, C. Arús, and A. Heerschap. MRS quality assessment in a multicentre study on MRSbased classification of brain tumours. NMR in Biomedicine, 21(2):148-158, 2008.

[VGGT ${ }^{+}$09] J. Vicente, J. García-Gómez, S. Tortajada, E. Fuster-Garcia, A. Capdevila, A. Peet, B. Celda, and M. Robles. Agefiltered mrs classifier to overcome the differences in childhood and adulthood brain tumours. In Proceedings 17th Scientific Meeting, International Society for Magnetic Resonance in Medicine, Honolulu, page 981, 2009. 
[VRGN ${ }^{+}$09] A. Vellido, E. Romero, F. F. González-Navarro, L. A. Belanche-Muñoz, M. Julií-Sapé, and C. Arús. Outlier exploration and diagnostic classification of a multi-centre 1h-mrs brain tumour database. Neurocomput., 72(1315):3085-3097, August 2009.

[VRJS $^{+}$12] A. Vellido, E. Romero, M. Julià-Sapé, C. Majós, A. Moreno-Torres, , and C. Arús. Robust discrimination of glioblastomas from metastatic brain tumors on the basis of single-voxel proton mrs. NMR in biomedicine, 25(6):819$828,2012$.

[VSVHVH01] L. Vanhamme, T. Sundin, P. Van Hecke, and S. Van Huffel. MR spectroscopy quantitation: a review of time-domain methods. NMR in Biomedicine, 14(4):233-246, 2001.

[VTFG $^{+}$12] J. Vicente, S. Tortajada, E. Fuster-Garcia, J. M. GarcíaGómez, and M. Robles. An audit method suited for decision support systems for clinical environment. In 6th IEEE International Conference on Intelligent Systems (IS'12), Sofia, Bulgaria, September 2012. Accepted. In press.

$\left[\mathrm{VVV}^{+} 96\right]$ A. Van den Boogaart, P. Van Hecke, S. Van Huffel, S. Graveron-Demilly, D. Van Ormondt, and R. de Beer. Mrui: a graphical user interface for accurate routine MRS data analysis. In Book of Abstracts 13th Annual Scientific Meeting of the European Society for Magnetic Resonance in Medicine and Biology, pages 12 - 15. Springer, Sep 1996.

[WA04] M. N. Wernick and J. N. Aarsvold. Emission tomography: the fundamentals of PET and SPECT. Elsevier Academic Press, 2004.

[WRK $\left.{ }^{+} 11\right]$ M. Wilson, G. Reynolds, R. A. Kauppinen, T. N. Arvanitis, and A. C. Peet. A constrained least-squares approach to the automated quantitation of in vivo $1 \mathrm{H}$ magnetic resonance spectroscopy data. Magnetic Resonance in Medicine, 65(1):1-12, January 2011.

[WW97] S. M. Wright and L. L. Wald. Theory and application of array coils in MR spectroscopy. NMR in Biomedicine, 10(8):394-410, 1997.

[ZJ98] W. Zong and D. Jiang. Automated ECG rhythm analysis using fuzzy reasoning. Computers in Cardiology, 25:6972, 1998.

[ZPAM $^{+}$05] Q. Zhao, P. Patriotis, F. Arias-Mendoza, R. Stoyanova, and T. R. Brown. An Interactive Software for 3D Chemical Shift 
Imaging Data Analysis and Real Time Spectral Localization and Quantification. In Book of Abstracts: International Conference on Magnetic Resonance in Medicine, Florida, 2005. 
